\title{
XENON-INDUCED SPATIAL INSTABILITIES IN
}

LARGE PRESSURIZED WATER REACTORS

AEC Coneract AT $(30-1)-3680$

Claude G. Poncelet

Alan M. Christie

March 1968

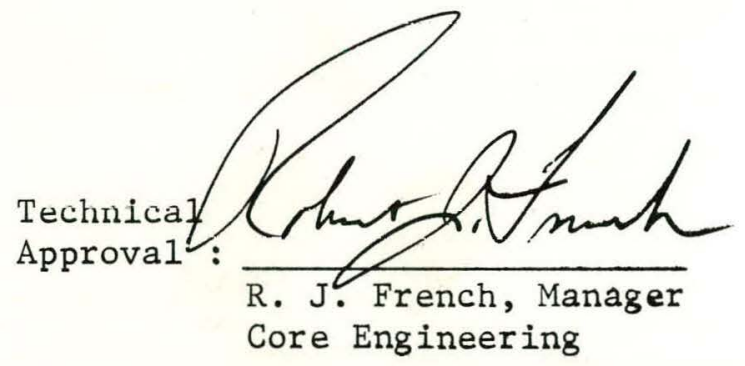

Project Approva1 : Core Engineering

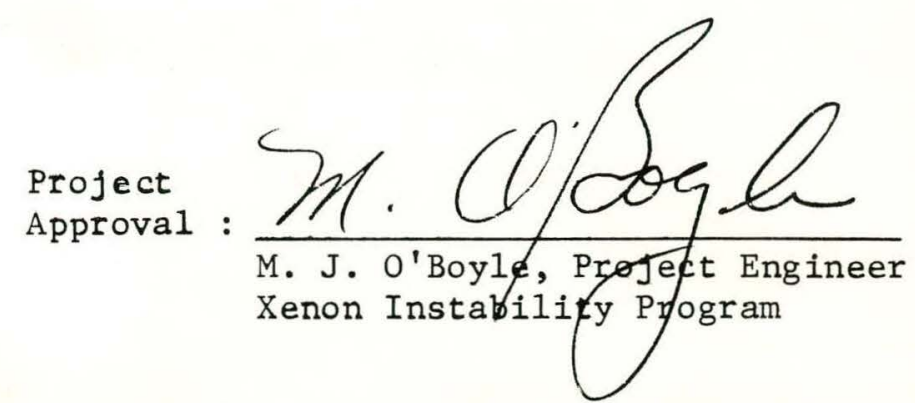

\footnotetext{
Westinghouse Electric Corporation

Atomic Power Divisions

P. O. Box 355

Pittsburgh, Pennsylvania 15230
} 


\section{DISCLAIMER}

This report was prepared as an account of work sponsored by an agency of the United States Government. Neither the United States Government nor any agency Thereof, nor any of their employees, makes any warranty, express or implied, or assumes any legal liability or responsibility for the accuracy, completeness, or usefulness of any information, apparatus, product, or process disclosed, or represents that its use would not infringe privately owned rights. Reference herein to any specific commercial product, process, or service by trade name, trademark, manufacturer, or otherwise does not necessarily constitute or imply its endorsement, recommendation, or favoring by the United States Government or any agency thereof. The views and opinions of authors expressed herein do not necessarily state or reflect those of the United States Government or any agency thereof. 


\section{DISCLAIMER}

Portions of this document may be illegible in electronic image products. Images are produced from the best available original document. 


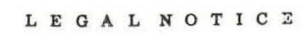

This report was prepared as an account of Government sponsored work. Nelther the United states, nor the Commission, nor ang porsonecting on behalf of the Commisaton:

A. Makes any warranty or representation, expressed or 1 mplied, with respect to the accuracy, completeness, or usefulness of the information contalned in this report, or that the use of any information, apparatus, method, or process disclosed in this report may uot lafinge privatelg oried rights; or

B. As Bumes any liabilitios with respeot to the use of, or for damages resulting from the use of any information, apparetus, method, or process diocloood in this roport.

As used in the above, "Person acting on behalf of the commisaton" includes any employee or contractor of the commission, or employee of auch

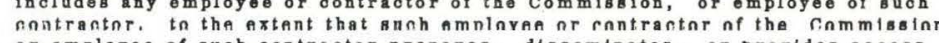
or employee of such contractor prepares, disseminates, or provides access to, any information pursuant to hisemployment or oontraot with the Commission, or his employment with ach contractor. 
EXTERNAL DISTRIBUTION

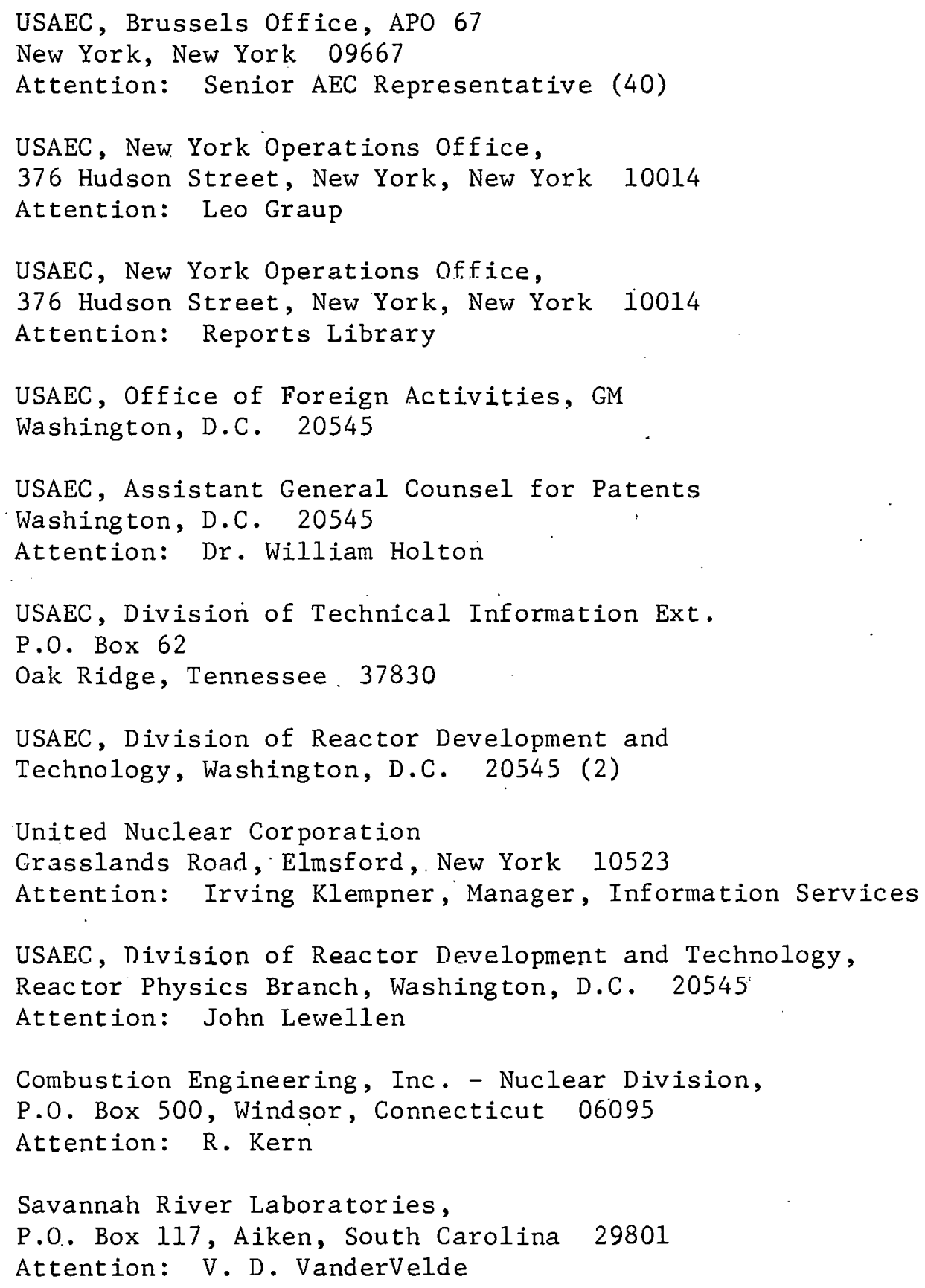




$\begin{array}{ll}\text { J. E. Alsina } & \text { J. D. McGaugh (5) } \\ \text { H. N. Andrews } & \text { D. L. Miller (5) } \\ \text { B. H. Axelson } & \text { G. H. Minton } \\ \text { G. H. Bantley (15) } & \text { T. J. Mitlo } \\ \text { R. F. Barry } & \text { N. R. Nelson } \\ \text { E. S. Beckjord } & \text { M. J. O'Boyle (6) } \\ \text { A. A. Bishop } & \text { W. L. Orr } \\ \text { J. O. Cermak } & \text { D. J. Povejsil } \\ \text { I. Chajson } & \text { V. Rajagopal } \\ \text { A. Christie (6) } & \text { R. E. Richards } \\ \text { I. H. Coen } & \text { R. E. Schrieber } \\ \text { P. Cohen } & \text { A. A. Simmons } \\ \text { R. J. Creagan } & \text { R. W. Steer } \\ \text { P. G. DeHuff } & \text { T. Stern } \\ \text { G. H. Farbman } & \text { L. E. Strawbridge } \\ \text { J. M. Gallagher } & \text { J. J. Taylor } \\ \text { H. W. Graves } & \text { D. E. Thorn } \\ \text { P. B. Haga } & \text { L. S. Tong } \\ \text { O. M. Hauge } & \text { R. A. Wiesemann } \\ \text { J. C. R. Kelly } & \text { P. M. Wood } \\ \text { F. Kramer } & \text { J. H. Wright } \\ \text { F. L. Langford } & \text { TIC (3) } \\ \text { W. Lester } & \end{array}$


The characteristics of free-running xenon-induced spatial oscillations in large pressurized water reactors ( $P W R^{\prime} s$ ) are investigated both from a phenomenological point of view and from the standpoint of the theoretical and calculational methods employed in their analysis. The digital simulation of spatial xenon instabilities with multidimensional, multi-group diffusion theory programs is investigated in detail. An extensive study of the effect of a finite, non-zero time-step length on calculated stability characteristics is presented, including a set of correlations which permits the extrapolation of digital calculations to effectively zero time-step length. The sensitivity of the digital results to the spatial mesh length, the energy mesh, and the treatment of temperature feedback effects is investigated. The effect of a non-zero flux convergence criterion on spatial xenon oscillations is investigated. A comparison is made of modal theory results and digital simulation calculations. The space-dependent transfer function formalism is employed in a number of analyses, including the effect of delayed neutrons and the effect of finite temperature feedback time-lags.

The space-time characteristics of xenon-induced instabilities in the twodimensional plane perpendicular to the direction of coolant flow are studied for a large variety of core sizes, power distributions and temperature feedback effects. The effect of perturbation size and location is evaluated. Extensive parametric and sensitivity analyses are performed, based on a modal expansion method. Parameters investigated are core size, power distribution, power level, power coefficient, fuel enrichment, Xe-135 absorption cross section, I-135 fission yield and direct Xe-135 fission yield: Parametric calculations with one- and two-dimensional diffusion theory programs are performed to corroborate. the modal theory results. 
TABLE OF CONTENTS

Section

$\underline{\text { Title }}$

$\underline{\text { Page }}$

ABSTRACT

iv

LIST OF ILLUSTRATIONS

vii

LIST OF TABLES

xiii

1.0 INTRODUCTION

$1-1$

2.0 THEORY AND CALCULATIONAL METHODS 2-1

2.1 GENERAL 2-1

2.2 THE DESCRIBING EQUATIONS 2-2

2.2.1 The Neutron Flux Equations 2-2

2.2.2 The Xe-135 and I-135 Equations 2-4

2.2.3 The Temperature Feedback Equations 2-4

2.3 DIGITAL SIMULATIONS . 2-6

2.3.1 Description and Comparison of Codes ZEST and TURBO* 2-7

2.3.2 Effect of a Finite Time-Step Length on Calculated Stability Characteristics $2-10$

2.3.3 Effect of Finite Flux Convergence Criterion 2-31

2.3.4 Effect of a Finite Spatial Mesh Size 2-33

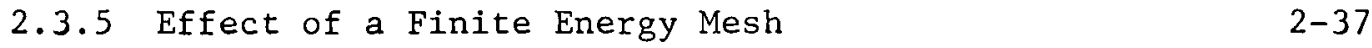

2.3.6 Treatment of Feedback Effects .2-38

2.3.7 Effect of Delayed Neutrons 2-42

2.3.8 Modified Procedures 2-44

2.4 MODAL ANALYSIS $2-47$

2.4.1 Geometric and Material Modes 2-48

2.4.2 Complex Natural Modes 2-59

2.5 SPACE-DEPENDENT TRANSFER FUNCTIONS-SPARTA CALCULATIONS 2-60 
TABLE OF CONTENTS (Continued)

$\underline{\text { Section }}$

$\underline{\text { Title }}$

Page

3.0 SPACE-TIME CHARACTERISTICS OF XENON-INDUCED OSCILLATIONS

IN LARGE PWR'S

$3-1$

3.1 SPATIAL CHARACTERISTIC'S UF USLILLATIÜN IN 'L'HL' $X-Y$ ' T'LAITT

32

3.1.1 Diametral Oscillations

$3-2$

3.1.2 Effect of Initial Power Distribution

$3-3$

3.1.3 Effect of Core Size

$3-16$

3.1.4 Effect of Leaving the Perturbation in the Core

3-16

3.2 INFLUENCE OF THE PERTURBATION LOCATION

$3-21$

3.3 INFLUENCE OF MAGNITUDE OF THE PERTURBATION

$3-30$

3.4 EFFECT OF VERY SMALL PERTURBATIONS ON CORE STABILITY

$3-40$

3.5 EFFECT OF TEMPERATURE FEEDBACKS

$3-45$

3.6 EFFECT OF NON-LINEARITIES IN CORE STABILITY

$3-50$

4.0 SENSITIVITY AND PARAMETRIC ANALYSES $\quad$ 4-1

4.1 MODAL ANALYSES 4-2

4.2 NUMERICAL CALCULATIONS 4-27

4.2.1 Effect of Variation of Power Density 4-27

4.2.2 Effect of Equilibrium Power Distribution . 4-31

4.2.3 Effect of Core Size 4-36

4.2.4 Effect of Varying Certain Core Parameters 4-46

5.0 SUMMARY AND CONCLUSIONS. $5-1$

REFERENCES $\quad$ R-1

APPENDIX A Core Descriptions $\quad \Lambda-1$

APPENDIX $B \quad$ Definition of the Stability Index B-1

APPENDIX $C \quad$ Stability Index Tables C-1

APPENDIX D Description of Program ZEST -- FORTRAN IV Code D-1

Acknowledgement 


\section{LIST OF ILLUSTRATIONS}

Variation of Xenon-Induced Spatial Stability Characteristics with Time-Step Length in Digital

Calculations (11-Foot Core, 1-Dimensional Calculation)

Variation of Xenon-Induced Spatial Stability Characteristics with Time-Step Length in Digital

Calculations (12-Foot Core, 1-Dimensional Calculation) 2-13

2-3 Variation of Xenon-Induced Spatial Stability

Characteristics with Time-S tep Length in Digital

Calculations (11-Foot Core, 2-Dimensional Calculation) 2-14

2-4 Comparison of Exact and Approximate Correlations of SLability Index as a Function of Time-Step Length

2-5 Variation of Xenon-Induced Spatial Stability Characteristics with Time-Step Length in Digital Calculations ( $84.5 \mathrm{w} / \mathrm{cc})$

2-6 Variation of Xenon-Induced Spatial Stability Characteristics with Time-Step Length in Digital Calculations (106 w/cc)

2-7 Comparison of Correlation from Equation (2.56) and Digital Calculations

Comparison of Theoretical and Calculated. Value of $\langle A\rangle$ as a Function of Power Level

Effect of Time-Step Length on Spatial Stability Index

Effect of Spatial Mesh Size on Xenon-Induced Oscillations

Effect of Spatial Mesh Size on Power Distribution

2-12 Effect of Temperature Time Lags on Xenon-Induced Instability

2-13 Spatial Distribution of Thermal Flux Variations (10-Foot Core)

2-14 Spatial Distribution of Excess Xenon Concentration (10-Foot Core) 


\section{LIST OF ILLUSTRATIONS (Continued)}

Figure

2-16

2-17

$2-18$

3-1

3-2

3-3

$3-4$

3-5

3-6

3-7

3-8

3-9

3-10

3-11

3-12

$3-13$

$3-14$

3-15

$3-16$
Title

Page

Spatial Distribution of Excess Xenon Concentration (11-Foot Core)

$2-54$

Effect of Spatial Dependence of the Temperature Feedback

$2-57$

$2-58$

and Modal Expansion Analysis

$3-4$

Worth of $1 \times 10^{-4}$

$3-5$

28 Hours after Removal of Perturbation

$3-5$

36 Hours after Removal of Perturbation

Perturbation,

Contour Power Distribution for 1/10 of Perturbation, 52 Hours after Removal of Perturbation

$3-5$

$3-7$

$3-8$

Initial Power Distribution for Checkerboard Core

$3-9$ after Removal of Perturbation

Power Distribution for Checkerboard Core, 32 Hours after Removal of Perturbation

Contour Power Plot for Checkerboard Core, 16 Hours after Removal of Perturbation

$3-11$

Contour Power Plot for Checkerboard Core, 28 Hours after Removal of Perturbation

Initial Power Distribution for Triple Peak Core Number 1

$3-12$

Initial Power Distribution for Triple Peak Core Number 2

$3-13$

Contour Power Plot for Triple Peak Core Number 1, 8 Hours after Removal of Perturbation

Contour Power Plot for Triple Peak Core Number 1, 16 Hours after Removal of Perturbation

Contour Power Plot for Triple Peak Core Number 1, 28 Hours after Removal of Perturbation

Contour Power Plot for Triple Peak Core Number 2, 8 Hours after Removal of Perturbation 


\section{LIST OF ILLUSTRATIONS (Continued)}

\begin{tabular}{|c|c|}
\hline Figure & Title \\
\hline $3-17$ & $\begin{array}{l}\text { Contour Power Plot for Triple Peak Core Number 2, } \\
16 \text { Hours after Removal of Perturbation }\end{array}$ \\
\hline $3-18$ & $\begin{array}{l}\text { Contour Power Plot for Triple Peak Core Number 2, } \\
28 \text { Hours after Removal of Perturbation }\end{array}$ \\
\hline $3-19$ & $\begin{array}{l}\text { Contour Power Distribution for 9-Foot Core, } 20 \text { Hours } \\
\text { after Removal of Perturbation }\end{array}$ \\
\hline $3-20$ & $\begin{array}{l}\text { Contour Power Distribution for } 9 \text {-Foot Core, } 32 \text { Hours } \\
\text { after Removal of Perturbation }\end{array}$ \\
\hline $3-21$ & $\begin{array}{l}\text { Contour Power Distribution for 9-Foot Core, } 44 \text { Hours } \\
\text { after Removal of Perturbation }\end{array}$ \\
\hline $3-22$ & $\begin{array}{l}\text { Steady-Stațe Power Distribution along } 0^{\circ}-180^{\circ} \text { Axis } \\
\text { for } 9 \text {-Foot Core }\end{array}$ \\
\hline $3-23$ & $\begin{array}{l}\text { Xenon-Induced Oscillations for Perturbation Left in } \\
\text { One Side of the Core. }\end{array}$ \\
\hline $3-24$ & $\begin{array}{l}\text { Contour Power Distribution for Perturbation Left in } \\
13 \text { Hours after Insertion of Pcrturbation }\end{array}$ \\
\hline $3-25$ & $\begin{array}{l}\text { Contour Power Distribution for Perturbation Left in } \\
21 \text { Hours after Insertion of Perturbation }\end{array}$ \\
\hline $3-26$ & $\begin{array}{l}\text { Contour Power Distribution for Perturbation Left in } \\
29 \text { Hours after Insertion of Perturbation }\end{array}$ \\
\hline $3-27$ & Xenon-Induced Oscillations along a $45^{\circ}-225^{\circ}$ Axis \\
\hline $3-28$ & $\begin{array}{l}\text { Xenon-Induced Oscillations along a } 221 / 2^{\circ}-2471 / 2^{\circ} \\
\text { Axis }\end{array}$ \\
\hline $3-29$ & $\begin{array}{l}\text { Contour Power Plot of Xenon Oscillations -- Steady-State } \\
\text { Flux Distribution for Full Core Calculation }\end{array}$ \\
\hline $3-30$ & $\begin{array}{l}\text { Contour Power Plot of Xenon Oscillations -- Power } \\
\text { Distribution } 4 \text { Hours after Removal of Perturbations }\end{array}$ \\
\hline $3-31$ & $\begin{array}{l}\text { Contour Power Plot of Xenon Oscillations -- Power } \\
\text { Distribution } 12 \text { Hours of after Removal of Perturbations }\end{array}$ \\
\hline $3-32$ & $\begin{array}{l}\text { Contour Power Plot of Xenon Oscillations -- Power } \\
\text { Distribution } 24 \text { Hours after Removal of Perturbations }\end{array}$ \\
\hline $3-33$ & $\begin{array}{l}\text { Effect of Perturbation Location on Xenon-Induced } \\
\text { Oscillations (Cases } i \text { and iv) }\end{array}$ \\
\hline $3-34$ & $\begin{array}{l}\text { Effect of Perturbation Location of Xenon-Induced } \\
\text { Oscillations (Cases } i i \text { and } i i i \text { ) }\end{array}$ \\
\hline $3-35$ & $\begin{array}{l}\text { Effect of Perturbation Location Power Distribution, } \\
28 \text { Hours after Perturbation }\end{array}$ \\
\hline
\end{tabular}




\begin{tabular}{|c|c|c|}
\hline Figure & Title & Page \\
\hline $3-36$ & $\begin{array}{l}\text { Contour Power Plot for Xenon-Induced Oscillations after } \\
\text { Removal of First Perturbation }\end{array}$ & $3-31$ \\
\hline $3-37$ & $\begin{array}{l}\text { Contour Power Plot for Xenon-Induced Oscillations after } \\
\text { Removal of Second Perturbation }\end{array}$ & $3-31$ \\
\hline $3-38$ & $\begin{array}{l}\text { Contour Power Plot for Xenon-Induced Oscillations, } 12 \\
\text { Hours after Removal of Second Perturbation }\end{array}$ & $3-32$ \\
\hline $3-39$ & $\begin{array}{l}\text { Contour Power Plot for Xenon-Induced Oscillations, } 20 \\
\text { Hours after Removal of Second Perturbation }\end{array}$ & $3-32$ \\
\hline $3-40$ & $\begin{array}{l}\text { Contour Power Plot for Xenon-Induced Oscillations, } 28 \\
\text { Hours after Removal of Second Perturbation }\end{array}$ & $3-33$ \\
\hline $3-41$ & $\begin{array}{l}\text { Contour Power Plot for Xenon-Induced Oscillations, } 40 \\
\text { Hours after Removal of Second Perturbation }\end{array}$ & $3-33$ \\
\hline $3-42$ & $\begin{array}{l}\text { Precession of Xenon-Induced Power Peak in Full Core } \\
\text { at Full Power }\end{array}$ & $3-34$ \\
\hline $3-43$ & $\begin{array}{l}\text { Precession of Xenon-Induced Power Peak in Full Core } \\
\text { at Half Power }\end{array}$ & $3-35$ \\
\hline $3-44$ & $\begin{array}{l}\text { Xenon-Induced Oscillations for a Poison Reactivity } \\
\text { Worth of } 3 \times 10^{-4}\end{array}$ & $3-37$ \\
\hline $3-45$ & $\begin{array}{l}\text { Xenon-Induced Oscillations for a Poison Reactivity } \\
\text { Worth of } 6 \times 10^{-4} \text {. }\end{array}$ & $3-38$ \\
\hline $3-46$ & $\begin{array}{l}\text { Digital Simulation of Xenon-Induced Spatial Oscillations } \\
\text { with Very Small Perturbation (Perturbed Source Guess) }\end{array}$ & $3-41$ \\
\hline $3-47$ & $\begin{array}{l}\text { Digital Simulation of Xenon-Induced Spatial Oscillations } \\
\text { with Very Small Perturbation (Perturbed Source Guess -- } \\
\text { Condensed Power Density Scale) }\end{array}$ & $3-43$ \\
\hline $3-48$ & $\begin{array}{l}\text { Digital Simulation of Xenon-Induced Spatial Oscillations } \\
\text { with Very Small Perturbation (Normal Source Guess) }\end{array}$ & $3-44$ \\
\hline $3-49$ & $\begin{array}{l}\text { Steady-State Power Distribution for Dished and Normal } \\
\text { 11-Foot Core with Fuel Temperature Feedback }\end{array}$ & $3-46$ \\
\hline $3-50$ & $\begin{array}{l}\text { Xenon-Induced Oscillations with and without Fuel } \\
\text { Temperature Feedback }\end{array}$ & $3-47$ \\
\hline $3-51$ & $\begin{array}{l}\text { Xenon-Induced Oscillations with and without Doppler } \\
\text { Feedback (Standard Core) }\end{array}$ & $3-48$ \\
\hline $3-52$ & $\begin{array}{l}\text { Effect of Variation of Fuel Temperature Feedback on } \\
\text { Diametral Xenon-Induced Osci.1.1ations }\end{array}$ & $3-51$ \\
\hline $3-53$ & Effect of Moderator Density Feedback on Thermal Gain & $3-52$ \\
\hline $3-54$ & $\begin{array}{l}\text { One-Dimensional Simulation of Azimuthal (Diametral) } \\
\text { Oscillations }\end{array}$ & $3-53$ \\
\hline
\end{tabular}




\section{LIST OF ILLUSTRATIONS (Continued)}

Figure

Title

Page

3-55 Spatial Power Distribution, 40 Hours after Removal of Perturbation for 15-Foot Core

3-56 Contour Power Plot, 24 Hours after Perturbation for $2 / 3$ Power

3-57 Contour Power Plot, 24 Hours after Perturbation for $4 / 3$ Power

$4-1$

Variation of Stability Index with U-235 Enrichment -Modal Analysis

Varlation of Stability with Core Diameter -- Modal

Analysis

Variation of Stability Index with Average Power Density -Modal Analysis

Variation of Stability Index with Power Distribution -Modal Analysis

Variation of Stability Index with Net Power Coefficient -. Modal Analysis

Variation of Stability Index with I-135 Yield -- Modal Analysis

Variation of Stability Index with Xenon Absorption Cross Section -- Modal Analysis

Variation of Stability Index with Direct XE-135 Yield -Modal Analysis

Variation of Oscillation Period with U-235 Enrichment -Modal Analysis

Variation of Oscillation Period with Core Diameter -Modal Analysis

Variation of Oscillation Period with Power Density -Modal Analysis

Variation of Oscillation Period with Power Distribution -Modal Analysis

4-13 Variation of Oscillation Period with Power Coefficient -Modal Analysis

4-14 Variation of Oscillation Period with Iodine Yield -Modal Analysis

4-15 Variation of Oscillation Period with Xenon-Absorption Cross Section -- Modal Analysis 


\section{LIST OF ILLUSTRATIONS (Continued)}

\begin{tabular}{|c|c|c|}
\hline igure & Title & Page \\
\hline $4-17$ & Xenon-Induced Oscillations at $2 / 3$ Power & $4-28$ \\
\hline $4-18$ & Xenon-Induced Oscillations at Full Power & $4-29$ \\
\hline $4-19$ & Xenon-Induced Oscillations at $4 / 3$ Power & $4-30$ \\
\hline $4-20$ & $\begin{array}{l}\text { Steady-State Power Distribution in 11-Foot Diameter } \\
\text { Core }\end{array}$ & $4-32$ \\
\hline $4-21$ & $\begin{array}{l}\text { Effect of Initial Power Distribution on Xenon-Induced } \\
\text { Oscillations -- Case A }\end{array}$ & $4-33$ \\
\hline $4-22$ & $\begin{array}{l}\text { Effect of Initial Power Distribution on Xenon-Induced } \\
\text { Oscillations -- Case B }\end{array}$ & $4-34$ \\
\hline $4-23$ & $\begin{array}{l}\text { Effect of Initial Power Distribution on Xenon-Induced } \\
\text { Oscillations -- Case D }\end{array}$ & $4-35$ \\
\hline $4-24$ & Xenon-Induced Oscillations for Triple Peak Core Number 1 & $4-37$ \\
\hline $4-25$ & Xenon-Induced Oscillations for Triple Peak Core Number 2 & $4-38$ \\
\hline $4-26$ & Xenon-Induced Oscillations for Checkerboard Core & $4-39$ \\
\hline $4-27$ & Xenon-Induced Oscillations for 9-Foot Core & $4-41$ \\
\hline $4-28$ & Xenon-Induced Oscillations for 10-Foot Core & $4-42$ \\
\hline $4-29$ & Xenon-Induced Oscillations for 13-Foot Core & $4-43$ \\
\hline $4-30$ & Xenon-Induced Oscillations for 15-Foot Core & $4-44$ \\
\hline $4-31$ & $\begin{array}{l}\text { Xenon-Induced Oscillations for } 10 \text { Percent Increase } \\
\text { in Absorption Cross-Section at a Poison Reactivity } \\
\text { Worth of } 1 \times 10^{-4}\end{array}$ & $4-48$ \\
\hline $4-32$ & $\begin{array}{l}\text { Xenon-Induced Oscillations for } 10 \text { Percent Reduction } \\
\text { in Absorption Cross-Section at a Poison Reactivity } \\
\text { Worth of } 1 \times 10^{-4}\end{array}$ & $4-49$ \\
\hline $4-33$ & $\begin{array}{l}\text { Xenon-Induced Oscillations for a } 10 \text { Percent Increase in } \\
\text { Total Yield at a Poison Reactivity Worth of } 9 \times 10^{-4}\end{array}$ & $4-50$ \\
\hline $4-34$ & $\begin{array}{l}\text { Xenon-Induced Oscillations for a } 10 \text { Percent Reduction in } \\
\text { Total Yield at a Poison Reactivity Worth of } 9 \times 10^{-4}\end{array}$ & $4-51$ \\
\hline $4-35$ & $\begin{array}{l}\text { Xenon-Induced Uscillations for a } 10 \text { Percent Increase in } \\
\text { Iodine Yield at a Poison Reactivity Worth of } 1 \times 10^{-4}\end{array}$ & $4-53$ \\
\hline $4-36$ & $\begin{array}{l}\text { Xenon-Induced Oscillations for a } 10 \text { Percent Reduction in } \\
\text { Iodine Yield at a Poison Reactivity Worth of } 1 \times 10^{-4}\end{array}$ & $4-54$ \\
\hline $4-37$ & Xenon-Induced Oscillations for a Direct Yield of 0.000 & $4-55$ \\
\hline $4-38$ & $\begin{array}{l}\text { Xenon-Induced Oscillations for a Direct Xenon Yield of } \\
0.004\end{array}$ & $4-56$ \\
\hline
\end{tabular}


2-1 Comparison of the Fast and Thermal Space-Dependent Transfer Functions

\section{LEGAL NOTICE}

This document was prepared under the sponsorship of the United States Atomic Energy Commission pursuant to the Joint Research and Development Program establighed by the Agreement for Cooperation signed November 8, 1938 between the Government of the United States of America and the European Atomic Energy Community (Euratom). Neither the United States, the U. S. Atomic Énergy Commisaion, the European Atomic Energy Community, the Euratom Commisaion, nor any person acting on behalf of either Commission:

A. Makes any warranty or representation, express or implied, with reepect to the accuracy, completeness, or usefulness of the information contained in this document, or that the use of any information, apparatus, method, or process disclosed in this document may not infringe privately owned rights; or

B. Aseumes any liabilities with respect to the use of, or for damages resulting from the use of any information, apparatus, method or proceso disclosed in this document.

As used in the above, "person acting on behalf of either Commission" includes any employee or contractor of either Commision or employee of such contractor lo Uhe extent that Buch employee or contractor or employee of such contractor prepares, handles, disseminates, or provides access to, any information pursuant to his employment or contract with either Commision or his employment with such contractor.

$7-7$ 


\section{SECTION 1}

\section{INTRODUCTION}

The trend toward improved economics and efficiency in Pressurized Water Reactors (PWR) design has led to increased core dimensions, higher flux levels, and more uniform power distributions during each fuel cycle. As has been known for some time, ${ }^{[1,2,3]}$ each of these improvements tends to make the core less stable toward spatial flux oscillations associated with Xe-135 redistribution in the core. The period of oscillation of xenon-induced spatial instabilities is long, relative to control mechanism or operator reaction time, being of the order of 28 hours in a large PWR. It has been suggested from the start $[3,4]$ that the control of spatial xenon oscillations with localized movable absorbers. should present no operational problems. Control calculations and operational experience have both indicated the validity of this premise. $[5,6]$ As was originally postulated by Randall and St. John, [3] it is more practical and economical to design for the detection and control of spatial xenon instabilities, rather than design an inherently stable core. The study of xenoninduced spatial oscillation in large PWR's has, therefore, a two-fold purpose. First, one is concerned with establishing the core's stability characteristics in terms of space and time. In this sense; one is not concerned with showing that a particular core is inherently stable, thus threshold analyses are not usually involved. Second, one is ultimately concerned with the design problem of detection and control of spatial xenon instabilities.

This report is devoted to the study of spatial stability characteristics in large PWR's. Such an analyșis must naturally include a detailed evaluation of the calculational methods which form the basis of the analysis. This analysis is closely related to the detection and control problem, in that a knowledge of the space-time characteristics of the oscillation must form the basis for any detection and control study, and in that the evaluation related to computational methods -- particularly to digital simulation -- is generally valid for control calculations. 
Because the subject of xenon oscillations is very comprehensive, it has been necessary to limit the analyses within a well defined domain. Only those oscillations which arise as a result of xenon redistribution are considered. In this context, the nuclear core is considered apart from the entire system; that is, inlet conditions to the core are assumed constant in time, and the analysis is restricted to the effect of xenon redistribution on the local flux or power density in the core. In general, only spatial oscillation will be considered, with the assumption that the fundamental flux shape is adequately controlled at all times. As far as stability characteristics are concerned, the analysis is restricted to the study of spatial oscillations in a plane perpendicular to the direction of coolant flow. That is, axial oscillations are not explicitly considered in this report, as they have been studied by Strawbridge, et al.[5] It should be noted, however, that much of the analysis devoted to computational methods has implications for spatial xenon oscillations in general. Finally, it is not the purpose of this report to study the stability characteristics of a particularly PWR design, although reallstic design data and configurations of large PWR's are used in the analysis.

The report is broadly divided into four sections. Section 2 describes the equation of motion of interest to this study, and evaluates the adequacy of a number of calculational methods. Section 3 presents the evaluations of the spatial. stability characteristic in large PWR's, including temperature feedback effects. Section 4 includes results of parametric and sensitivity analyses. Each section has been written such that it is not essential for the reader to study all sections if his interest is restricted to one particular topic. 


\section{SECTION 2}

THEORY AND CALCULATIONAL METHODS

\subsection{GENERAL}

The various methods of calculation used in this work are described in this section. The approximations and limitations, as well as the fustification for the use of each method, are discussed.

Section 2.2 summarizes the equations of motion which describe the phenomenon of xenon-induced spatial instabilities in large PWR's. Section 2.3 discusses the direct numerical solution of these equations in space and time, using a digital computer. Section 2.4 discusses the use of modal expansions, and Section 2.5 discusses the use of the space-dependent transfer function. Both of these methods are based on linear theory, and are therefore restricted to small variations of the state variables about the same equilibrium point. Digital simulation has been selected as the basic tool for studying spatial xenon instabilities in large PWR's. This selection stems from a number of considerations. The major ones are:

1. the capability of representing complex core configuration in multidimensional geometries;

2. the capability of simulating realistic control actions;

3. the inclusion of non-linearities in the equation, including non-linear feedback effccts.

The main limitations of digital simulations reside in the errors introduced as a result of the approximations in the numerical treatment. These errors arise as a result of the use of a discrete mesh in both time and space. It is also generally true that a digital simulation gives less insight to the physical picture than does an analytic model. The iterative nature of the solution of the eigenvalue calculation performed at each time step also introduces a source of error due to the use of a finite (nonzero) convergence criterion. 
These effects usually impose real limitations in that the use of very fine meshes in time and space and of very tight convergence criteria leads to excessive computer time. These effects are discussed in detail in Section 2.3. Within the framework of linear theory, modal analysis has the advantage over digital simulation in that it does not involve a discretization in time. A major limitation of modal theory, however, is the difficulty of realistically representing both feedback and control effects. Furthermore, the representation of complex core geometries does require the performance of numerical calculations on a digital computer. The simple modal theory, based on expansion in material modes, has one advantage in that analytical expansion can be derived for the spatial stability characteristics of the core. These analytical expressions are used in this report to study the sensitivity of the stability characteristics to changes in core parameters and to basic physics data.

Again within the framework of linear theory, the space-dependent transfer function formulation offers advantages which are peculiar either to digital simulation or to modal analyses. Although the formulation shows the representation of realistic core geometries and feedback treatments, it does not depend on a discretization of the time domain. In any case, for onedimensional geometries it is also a non-iterative process. A major limitation of the method lies in the representation of control actions.

\subsection{THE DESCRIBING EQUATIONS}

The state variables considered here are the neutron flux in space, energy and time, and the feedback variables. The feedback variables considered are the Xe-135 and I-135 atom concentrations, and the fuel and moderator temperatures. All analyses are restricted to a plane perpendicular to the direction of coolant flow.

\subsubsection{The Neutron Flux Equations}

The neutron flux distribution will be described by the two-group neutron diffusion equations. In general, the time-dependent neutron diffusion equations can be written in the form: 


$$
\begin{aligned}
& \nabla \cdot \mathrm{D}_{1}(\underline{r}, t) \nabla \phi_{1}(\underline{r}, t)-\Sigma_{a_{1}}(\underline{r}, t) \phi_{1}(\underline{r}, t)-\sum_{R}(\underline{r}, t) \phi_{1}(\underline{r}, t) \\
& +\lambda^{-1} \sum_{j}\left\{\left(1-\beta^{j}\right)\left[v \Sigma_{f_{1}}^{j}(\underline{r}, t) \phi_{1}(\underline{r}, t)+v \Sigma_{f_{2}}^{j}(\underline{r}, t) \phi_{2}(\underline{r}, t)\right]\right\} \\
& +\sum_{i} \lambda_{i} c_{i}(\underline{r}, t)-\Sigma_{p_{1}}(\underline{r}, t) \phi_{1}(\underline{r}, t)=\frac{1}{v_{1}} \frac{\partial \phi_{1}(\underline{r}, t)}{\partial t} \\
& \nabla \cdot D_{2}(\underline{r}, t) \nabla \phi_{2}(\underline{r}, t)-\Sigma_{a_{1}}(\underline{r}, t) \phi_{2}(\underline{r}, t)+\sum_{R}(\underline{r}, t) \phi_{1}(\underline{r}, t) \\
& -\sum_{\mu_{2}}(\underline{r}, t) \phi_{2}(\underline{r}, t)=\frac{1}{v_{2}} \frac{\partial \phi_{2}(\underline{r}, t)}{\partial t} \\
& \sum_{j} \beta_{i}^{j}\left[v \Sigma_{f_{1}}^{j}(\underline{r}, t) \phi_{1}(\underline{r}, t)+v \Sigma_{\underline{f}_{2}}^{j}(\underline{r}, t) \phi_{2}(\underline{r}, t)\right]-\lambda_{i} C_{i}(\underline{r}, t) \\
& =\frac{\partial C_{i}(\underline{r}, t)}{\partial t}
\end{aligned}
$$

where subscripts 1 and 2 refer to the fast and thermal group, subscript $i$ denotes the $i^{\text {th }}$ delayed neutron precursor, superscript $j$ denotes the $j^{\text {th }}$ fissionable isotope and $\Sigma_{\mathrm{P} 1}$ and $\Sigma_{\mathrm{P} 2}$ represent fast and thermal perturbation cross sections. The remaining notation is standard.

The diffusion coefficients and macroscopic. cross sections are treated as functions of time because of the feedback effects. It will be assumed that the change in Xe-135 concentration only affects the absorption cross section. Thus, we write

$$
\begin{aligned}
& \Sigma_{a_{1}}(\underline{r}, t)=\Sigma_{a_{1}}^{*}(\underline{r}, t)+N_{x}(\underline{r}, t) \sigma_{x_{1}}^{*}(\underline{r}) \\
& \Sigma_{a_{2}}(\underline{r}, t)=\Sigma_{a_{2}}^{*}(\underline{r}, t)+N_{x}(\underline{r}, t) \sigma_{x_{2}}^{*}(\underline{r})
\end{aligned}
$$


where $\Sigma_{a_{1}}^{*}$ and $\Sigma_{a_{2}}^{*}$ refer to xenon-free conditions (*denotes real part). The use of two energy groups is usually adequate for the calculation of steadystate power distribution at PWR's. Adequacy of a two-group treatment of spatial xenon-induced instabilities is briefly discussed in Section 2.3.5. The approximations to equations (2.1) through (2.3) in digital simulation, modal analyses and transfer function analyses are discussed in sections 2.3 through 2.5 .

\subsubsection{The Xe-135 and I-135 Equations}

The equations of motion of the Xe-135 and I-135 concentrations are

$$
\begin{gathered}
\frac{\partial N_{x}(\underline{r}, L)}{\partial t}=\lambda_{i}^{\prime} N_{i}(\underline{r}, t)+\sum_{j} y_{x}^{j}\left[\sum_{f_{1}}^{j}(\underline{r}, t) \phi_{1}(\underline{r}, t)+\sum_{f_{2}}^{j}(\underline{r}, t) \phi_{2}(\underline{r}, t)\right] \\
-\left[\sigma_{a_{1}}^{x}(\underline{r}) \phi_{1}(\underline{r}, t)+\sigma_{a_{2}}^{x}(\underline{r}) \phi_{2}(\underline{r}, t)+\lambda_{x}\right] N_{x}(\underline{r}, t) \\
\frac{\partial N_{i}(\underline{r}, t)}{\partial t}=-\lambda_{i} N_{i}(\underline{r}, t)+\sum_{j} y_{i}^{j}\left[\sum_{f_{1}}^{j}(\underline{r}, t) \phi_{1}(\underline{r}, t)+\sum_{f_{1}}^{j}(\underline{r}, t) \phi_{2}(\underline{r}, t)\right]
\end{gathered}
$$

The effect of Xe-135 on the cross sections is given by equations (2.4) and $(2.5)$.

\subsubsection{The Temperature Feedback Equations}

In a PWR, the major temperature feedback effects are: a) the Doppler effect accompanying a change in fuel temperature, and b) the moderator density changes. In terms of cross section variations, the fuel temperature affects primarily the fast removal and fast absorption cross sections, whereas the moderator density affects the fast removal and thermal absorption cross sections. In a core controlled by chemical shim, the variation of the thermal absorption cross section with moderator density, as a result of variations in boron density, is particularly important. 
The variations in time and space of the fuel and moderator temperatures in the plane perpendicular to the direction of coolant flow can be approximated by a simple model which assumes that the local temperatures are functions only of the local power density. Thus, the equations of motion for the fuel and moderator temperatures can be written in the form

$$
\begin{aligned}
& C_{f}(\underline{r}) \frac{\partial T_{f}(\underline{r}, t)}{\partial t}=p(\underline{r}, t)-h(\underline{r}) T_{f}(\underline{r}, t) \\
& C_{m}(\underline{r}) \frac{\partial T_{m}(\underline{r}, t)}{\partial t}=h(\underline{r}) T_{f}(\underline{r}, t)-U T_{m}(\underline{r}, t)
\end{aligned}
$$

where $C_{f}$ and $C_{m}$ are heat capacities of fuel and moderator, respectively, and $\mathrm{h}$ is the heat transfer coefficient of the coolant. In equations (2.8) and (2.9), $\mathrm{T}_{f}$ and $\mathrm{T}_{\mathrm{m}}$ are defined with respect to base values, which can be the core average moderator temperature and the inlet coolant temperature, respectively. Note that $C_{f}$ and $h$ are, in general, a function of the local heat flux such that the feedback equations (2.8) and (2.9) are non-linear equations.

It is sometimes useful to use the coolant enthalpy, rather than the coolant temperature, as the state variable. An equation similar to equation (2.9) can then be written to describe the variations in space and time of the coolant enthalpy.

Equations (2.8) and (2.9) can be written in the following useful form

$$
\begin{gathered}
\tau_{f} \frac{\partial T_{f}}{\partial t}=K_{f} p-T_{f} \\
\tau_{m} \frac{\partial T_{m}}{\partial t}=T_{f}-\frac{K_{f}}{K_{m}} T_{m}
\end{gathered}
$$

where $\tau_{f}$ and $\tau_{m}$ are the fuel and moderator time constants, and $K_{f}$ and $K_{m}$ are fuel and moderator gain constants:

$$
\tau_{f}=\frac{C_{f}}{h}
$$




$$
\begin{aligned}
& \tau_{m}=\frac{C_{m}}{h} \\
& K_{f}=\frac{1}{h} \\
& K_{m}=\frac{1}{U}
\end{aligned}
$$

Since the period of xenon oscillations is so long, fuel and moderator temperature time lags are effectively zero, i.e., $\tau_{f} * \tau_{m}-0$. Thus equations 2-10 and 2-11 can be written as

$$
\begin{aligned}
& T_{f}=K_{f}, P \\
& T_{m}=\frac{K_{m}}{K_{f}} T_{f}
\end{aligned}
$$

The first of these two equations is made use of in the program ZEST which is described in Section 2.3.1.2.

\subsection{DIGITAL SIMULATIONS}

As mentioned previously, the most realistic way of simulating the conditions in a reactor core is by using a computer mathematical model in which gcometrical shapes and other core details can be represented. In the present investigation, a one-dimensional and a two-dimensional code were employed. - In the first of these, ZEST was designed and programmed specifically for this xenon stability analysis, and as such, was very simple to use in this context. The second code, TURBO*, [26] was an already existing two-group depletion code. As a consequence of its being two-dimensional, it took considerably longer to run than ZEST. The TURBO* code has to iterate over the spatial mesh (the inner iterations) and over this must iterate on the eigenvalue (the outer iterations).

For certain calculations involving diametral oscillations, results from the two codes had good qualitative agreement. Under these conditions, therefore, the one-dimensional ZEST was used in preference to TURBO*: 
As with all digital solutions of differential equations, errors are unavoidably introduced due to the discrete nature of the difference equations representing the system. Apart from the finiteness of the spatial and temporal mesh lengths, errors are incurred from the finite convergence criteria for the eigenvalue and flux shape, and for the finite number of energy groups. These topics will all be discussed in subsequent sections with reference to xenon oscillations.

Also as discussed later, the results of a number of numerical calculations were compared with the corresponding analytic model so that the results of each might give a certain confidence level in the other.

\subsubsection{Description and Comparison of Codes ZEST and TURBO*}

The codes ZEST and TURBO* were both used to solve modified forms of equations (2.1) and (2.2). In neither case were delayed neutrons introduced through equation (2.3). As shown in Section 2.3:7 and as can also be seen from reference 8 , this approximation is quite valid in the context of xenon oscillations. ZEST, as with TURBO*, solyes the two-group form of the diffusion equations (equations 2.1 and 2.2) and in both cases, due to the long xenon time constants the right hand side of these equations were equated to zero. Thus, the two diffusion equations can be written in the form

$$
\begin{aligned}
& \nabla \cdot D_{1}(\underline{r}) \nabla \phi_{1}(\underline{r}, t)+\Sigma_{T}^{i}(\underline{r}, t) \phi_{i}(\underline{r}, t) \quad i=1,2 \ldots, K \\
= & \frac{x_{i}}{\lambda} \sum_{j}\left\{v \Sigma_{f}^{j}(r) \phi_{j}(\underline{r}, t)\right\}+\Sigma_{R}^{i-1}(r) \phi_{i-1}(\underline{r}, t),
\end{aligned}
$$

where $\mathrm{K}=$ number of groups

$$
\Sigma_{T}^{i}(\underline{r}, t)=\Sigma_{a}^{i}(\underline{r}, t)+\Sigma_{p}^{i}(\underline{r}, t)+\Sigma_{R}^{i}(r)+D_{i}(r) B^{2}
$$

$\Sigma_{a}^{i}(r, t)$, as defined in equations (2.4) and (2.5), is composed of a fraction depending upon the concentration of xenori, the complementary part being due 
to all other absorptions. This latter part, although time-varying as a result of fuel depletion, changes so slowly compared with the xenon absorption that it can be considered constant in time. Thus ZEST ignores these slowlyvarying isotopes, but, in TUBRO*, these minor depletion chains were accounted for. Both codes use a discrete time mesh in that between spatial calculations the flux, cross sections, etc. remain constant over the time interval that the xenon and iodine chains were depleted. These chains, because of their mathematical simplicity, were solved exactly on the assumption that for the depletion time, the flux remained constant. As will be shown in Section 2.3.2, however, this assumption is not strictly correct.

The expression $\Sigma_{p}^{i}(r, t)$ denotes the cross section for homogenized poison. After an equilibrium flux (with equilibrium xenon) has been established, a local poison is inserted for some time in order to distort the flux shape and consequently initiate oscillations. Once these oscillations are started, the poison is no longer required and is withdrawn.

\subsubsection{The TURBO* Code}

The description of the code given here contains only points which are relevant to this study, a fuller description of its operation can be found elsewhere.

The outer iteration scheme in TURBO* contains a flux renormalization which, in effect, keeps the power in the reactor constant and consequently suppresses the fundamental mode. In a spatial stability analysis this is important, as contamination from this mode would obscure the spatial xenon oscillation. In the treatment of fuel-temperature feedback; TURBO* considers the removal cross sections as constant and varies the fast absorption cross sections through the resonance escape probability $p$, which in turn is fitted to a function of the total core power. Hence the reactivity $k$, being a linear function of $p$, is held at its correct value. However, since removal to the thermal region is affected by power, the reaction rates in this region are not exact and the stability through the production of xenon will be affected. An estimate of this error is given in Section 2.3.6. Moderator temperature feedback is not accounted for in TURBO*. 


\subsubsection{The ZEST Code}

The ZEST code is a one-dimensional, two-group diffusion code specifically designed for use in the investigation of xenon instabilities. It was also used as a tool for testing and evaluating a variety of computational schemes, some of which are discussed in Section 2.3.8. The numerical scheme for solving the diffusion and xenon equations in ZEST is a modification of the finite difference scheme employed in SPARTA. [11] As such, the spatial mesh is swept across to determine the flux values for that particular iteration, this being performed simultaneously for the fast and thermal groups. The iterations are continued until both the eigenvalue and the flux across the core have converged to within specified limits. The code is set up to take a zero flux or. zero current boundary conditions, depending upon whether or not a symmetrical (slab) core is desired.

Because of the nature of the code, only the iodine and xenon chains are depleted. Since the equations defining these chains are solved analytically, they are accurate to within the error incurred in the assumption that the flux is independent of time across the time-step under consideration. ZEST has a relatively simple input in that the code automatically perturbs the core and follows the subsequent oscillations. The procedure followed by the code is described below:

a. computation of steady-state conditions, with equilibrium Xe-135 concentration

b. flux calculation with a poison inserted into the core and with the equilibrium Xe-135 concentration; the location and strength of the perturbation being on input data

c. calculation of the I-135 and Xe-135 concentration at a specified time after insertion of the perturbation; this time period being an input datum

d. flux calculation with the neutron poison inserted and the Xe-135 concentration obtained in (c) above

e. flux calculation with the poison removed and the Xe-135 concentration obtained in (c) above 
This series of calculations is followed by a specified number of time-steps; the duration of these time steps is constant and constitutes an input datum. The local feedback treatment employed in ZEST accounts for variations in fast removal as well as absorption cross sections. These cross sections are both fitted to second-order polynominals in fuel temperature which is, in turn, fitted to the same order of the polynominal in power. A detailed description of the mathematical model employed in ZEST is given in Appendix D.

The main justification for employing one-dimensional calculations, as opposed to multidimensional calculations, is obviously based on machine-time requirements. It should be noted, however, that for the mode of oscillation of interest to this study, one-dimensional calculations in slab geometry do afford a relatively realistic picture. As has been observed from two-dimensional calculations, ${ }^{[31,32]}$ the mode most readily excited in the $x-y$ plane corresponds to oscillations across a core diameter. Such oscillations can be simulated in one-dimensional slab geometry by using a reflected slab whose length corresponds to the actual length along a diameter of the core. The output edit for each time-step consists of the converged eigenvalue followed by the thermal and fast fluxes, fission source, power density, Xe-135 number density and fuel temperature.

\subsubsection{Effect of a Finite Time-Step Length on Calculated Stability Characteristics}

In digital simulations of xenon-induced oscillations using diffusion theory codes, the time-dependence of the spatial flux distribution is discretized by using a time mesh or a number of time-steps of non-zero lengths. In the majority of diffusion theory codes, the assumption is made that the spatial flux distribution is constant in time during the length of the time-step. It can be readily deducèd that for unstable cores somewhat removed from the stability threshold, a non-zexo time-step will result in observed or calculated oscillations which are relatively more stable than in the limit of a zero time-step length. Similarly, for stable cores somewhat removed from the stability threshold, the calculated oscillations will be relatively. less stable than in the limit of a zero time-step length. For example, 
when the flux of.a point is increasing, the assumption of a constant flux during the duration of the time-step results in an underestimate of the xenon concentration change.

Since diffusion theory calculations, particularly two- and three-dimensional calculations, are time consuming; economic considerations preclude the use of very short time-steps. Therefore, it is essential to ascertain the error introduced by the use of a finite time-step length. In the following paragraphs, numerical results are first displayed where the same core was studied with different time-step lengths. A theoretical analysis of the effect is next discussed which leads to an estimate of the error introduced by a non-zero time-step length. It is shown, at least for uncontrolled calculations and within the limitations of linear theory, that a simple correction can be made to digital calculations to account for the use of a non-zero time-step length.

\subsubsection{Numerical Evaluation}

Xenon-induced diametral oscillations in a standard 11-foot core, with nominal fuel temperature feedback, were simulated in the ZEST program with time-steps of one, two and four-hour duration. The resulting thermal flux oscillations are shown in Figure 2-1. Note that whereas the use of a fourhour time-step predicts spatial stability, the use of a shorter time-step length actually shows the core to be spatially unstable. It is observed that both the damping factor and the oscillation period are sensitive to the time-step length.

The oscillations in Figure 2-1 are of relatively small magnitude. Figure 2-2 shows a similar analysis for relatively large oscillations. The core in this instance is a one-dimensional slab, 12 feet in length, with uniform fuel (2.6 a/o U-235) and no temperature feedback. The sensitivity to the timestep length for both the damping factor and the oscillation period is again evident.

Figure 2-3 shows results for two-dimensional TURBO* calculations with twohour and four-hour time-steps. The standard 11-foot core had a flattened power distribution and no temperature feedback. The reactivity effect of 


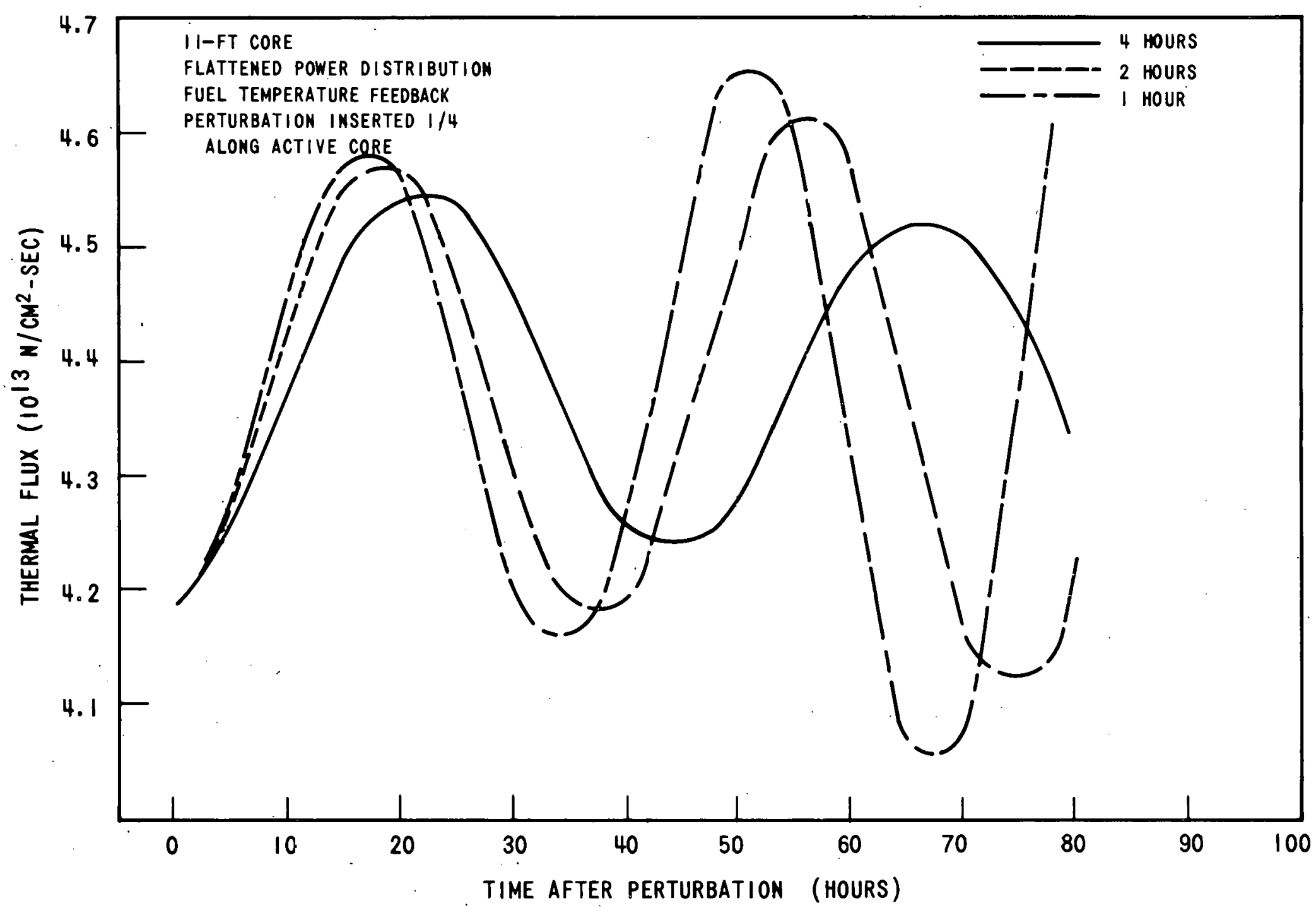

Figure 2-1. Variation of Xenon-Induced Spatial Stability Characteristics with Time-Step Length in Digital Calculations (11-Foot Core, 1-Dimensional Calculation) 


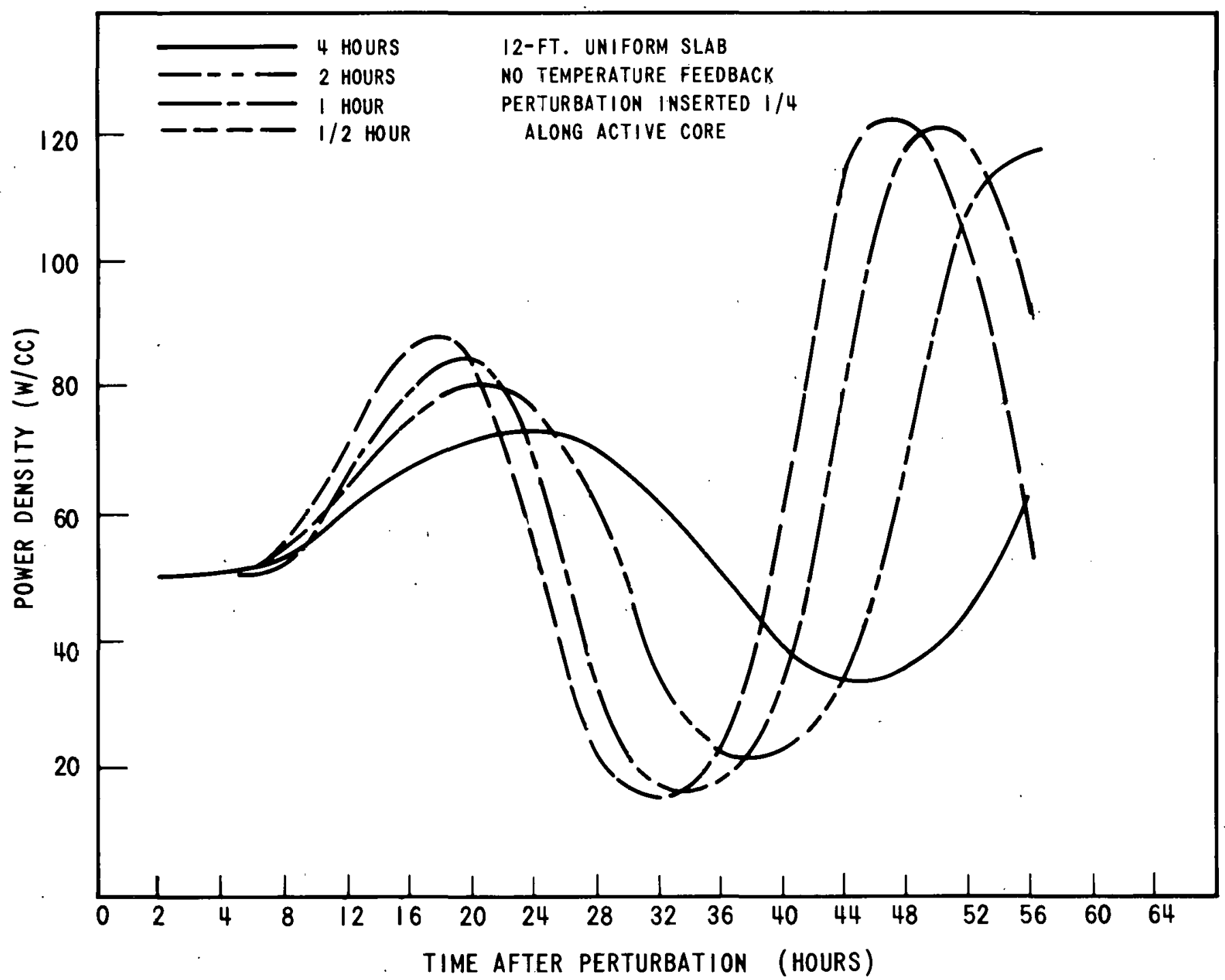

Figure 2-2. Variation of Xenon-Induced Spatial Stability Characteristics with Time-Step Length in Digital Calculations (12-Foot Core, 1-Dimensional Calculation) 


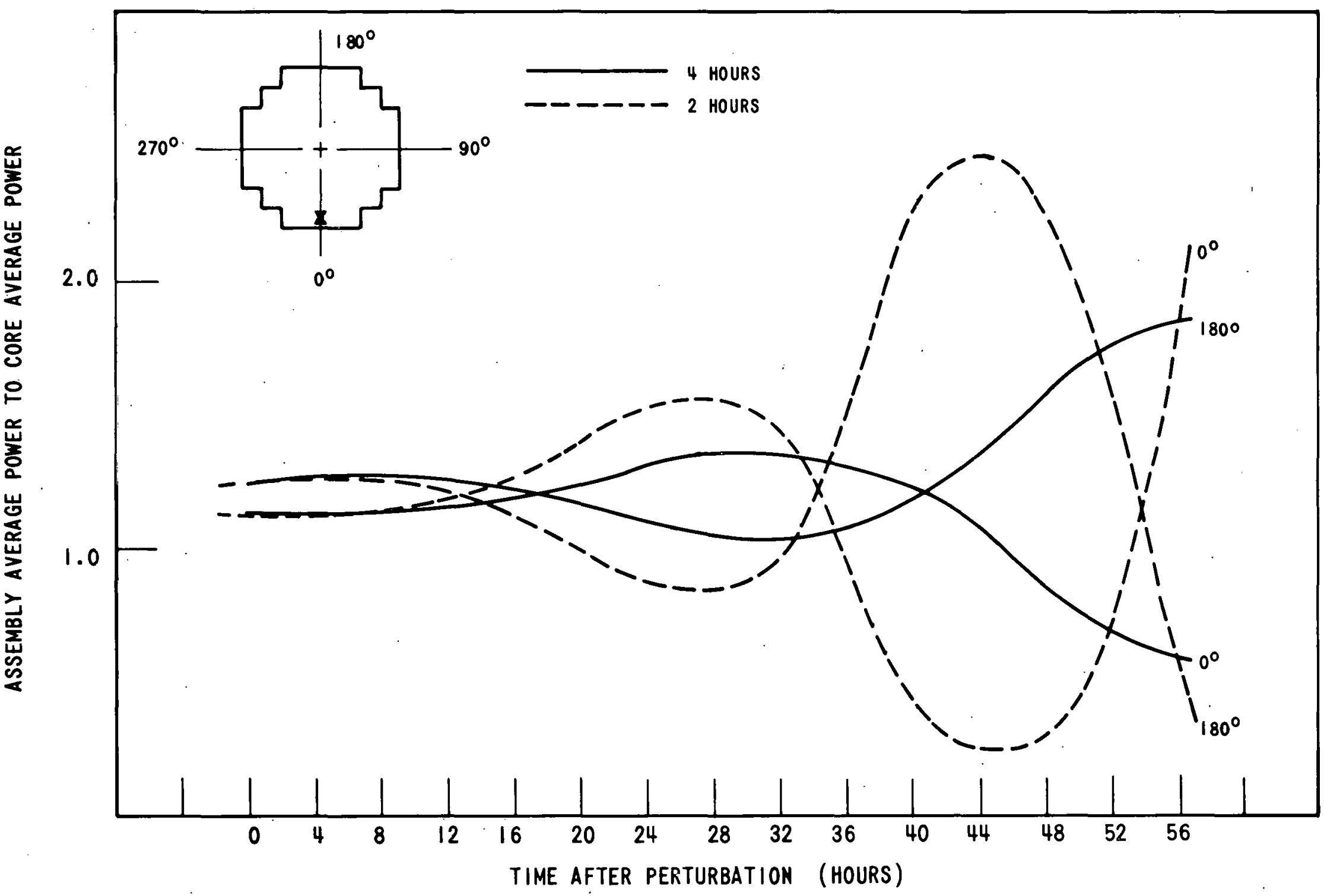

$\underline{\beth}$

Figure 2-3. Variation of Xenon-Induced Spatial Stability Characteristics with Time-Step Length in Digital Calculations (11-Foot Core, 2-Dimensional Calculation) 
the perturbation for the core was $1 \times 10^{-4}$. From Figures 2-1 through 2-3 it is evident that the sensitivity to the time-step length is greater for relatively less stable cores. Although the numerical results displayed here are in qualitative agreement with similar calculations published by Harris and Lacy [12] for a seed-blanket reactor, they are at variance with conclusions reached by Strawbridge [5] for slightly enriched PWR's. It is shown in the following paragraph, however, that the particular cases studied by Strawbridge fell in a portion of the stability plane where the sensitivity to the time-step length is a minimum.

\subsubsection{Analytical Evaluation}

Davison has published a theoretical investigation of the error introduced in digital calculations by the use of a non-zero time-step length. [13] In this section an analytical evaluation of the time-step length problem is presented which, although based on Davison's ideas, uses a different treatment of the spatial distributions than was assumed by Davison. In addition, simple correlations are derived for slightly enriched PWR's which can be used to estimate the error introduced by a non-zero time-step length.

In one-group theory, the describing equations for the time-dependent xenon problem are written in the form

$$
\begin{gathered}
\nabla \cdot D(\underline{r}) \nabla \phi(\underline{r}, t)-\Sigma_{a}(\underline{r}, t) \phi(\underline{r}, t)-N_{x}(\underline{r}, t) \sigma_{x} \phi(\underline{r}, t) \\
\left.+i \Sigma_{f}(\underline{r}) \phi \underline{r}, t\right)=0 \\
\frac{\partial N_{i}(\underline{r}, t)}{\partial t}=y_{i} \Sigma_{f}(\underline{r}) \phi(\underline{r}, t)-\lambda_{i} N_{i}(\underline{r}, t)
\end{gathered}
$$




$$
\begin{aligned}
\frac{\partial N_{x}(\underline{r}, t)}{\partial t} & =\lambda_{i} N_{i}(\underline{r}, t)+y_{x} \Sigma_{f}(\underline{r}) \phi(\underline{r}, t) \\
& -N_{x}(\underline{r}, t)\left[\sigma_{x} \phi(\underline{r}, t)+\lambda_{x}\right]
\end{aligned}
$$

The notation in equations (2.16) through (2.18) is standard. Temperature feedback is accounted for in $\Sigma_{a}(\underline{\underline{r}}, t)$. In the usual way, small variations of the dependent variables about their steady-state values are now introduced:

$$
\begin{gathered}
\phi(\underline{r}, t)=\phi_{0}(\underline{r})+\delta \phi(\underline{r}, t) \\
N_{i}(\underline{r}, t)=N_{i}(\underline{r})+\delta N_{i}(\underline{r}, t) \\
N_{x}(\underline{r}, t)=N_{x_{0}}(\underline{r})+N_{x}(\underline{r}, t) \\
\Sigma_{a}(\underline{r}, t)=\Sigma_{a}(\underline{r})+\delta \Sigma_{a}(\underline{r}, t)=\sum_{a}(\underline{r})+K_{a}(\underline{r}) \delta \phi(\underline{r}, t)
\end{gathered}
$$

The last of equations (2.19) is a representation of linear feedback. Substitution of equations (2.19) into equations (2.16) through (2.18), linearization of the resulting equations, and elimination of the steady-state equations lead to the following set of perturbed equations:

$$
\begin{gathered}
\nabla \cdot D \nabla \delta \phi-\Sigma_{a_{0}} \delta \phi-\mathrm{N}_{\mathrm{x}_{\mathrm{o}}} \sigma_{\mathrm{x}} \delta \phi+\nu \Sigma_{\mathrm{f}} \delta \phi-\mathrm{K}_{\mathrm{a}} \phi_{\mathrm{o}} \delta \phi-\phi_{0} \sigma_{\mathrm{x}} \delta \mathrm{N}_{\mathrm{x}}=0 \\
\frac{\partial \delta \mathrm{N}_{\mathrm{i}}}{\partial t}=\mathrm{y}_{\mathrm{i}}{ }^{\prime} \Sigma_{\mathrm{f}} \delta \phi-\lambda_{\mathrm{i}} \delta \mathrm{N}_{\mathrm{i}} \\
\frac{\partial \delta \mathrm{N}_{\mathrm{x}}}{\partial \mathrm{t}}=\lambda_{i} \delta \mathrm{N}_{\mathrm{i}}+\mathrm{y}_{\mathrm{x}} \Sigma_{\mathrm{f}} \delta \phi-\mathrm{N}_{\mathrm{x}} \sigma_{\mathrm{x}} \delta \phi-\delta \mathrm{N}_{\mathrm{x}}\left(\sigma_{\mathrm{x}} \phi_{\mathrm{o}}+\lambda_{\mathrm{x}}\right)
\end{gathered}
$$


where the space and time dependence were left out for clarity. The steady-state equation for the flux can be written

$$
L_{0} \phi_{0}=0
$$

where $L_{0}$ is the unperturbed, steady-state operator corresponding to equation (2.16). Equation (2.20) can be written in the form

$$
\left(\mathrm{I}_{0}-\mathrm{L}_{1}\right) \delta \phi=0
$$

where

$$
\mathrm{L}_{1}=\left(\mathrm{K}_{\mathrm{a}}+\sigma_{\mathrm{x}}\right) \phi_{0}
$$

A modal expansion of the flux perturbation is now assumed, of the form

$$
\delta \phi(\underline{r}, t)=\sum_{n} a_{n} e^{\omega_{n} t} \psi_{n}(\underline{r})
$$

where $\psi_{n}$ are the eigenvectors corresponding to the eigenvalues $\mu_{n}^{2}$ of the steady-state operator $\mathrm{L}_{0}$ :

$$
L_{0} \psi_{n}=\mu_{n}^{2} \psi_{n}
$$

Note that $\mu_{0}=0$, and $\psi_{0}=A \phi_{0}$ where $A$ is a constant.

The eigenvectors $\psi_{n}$ are orthonormal:

$$
\int \underline{d}_{\underline{r}} \psi_{\mathrm{n}} \psi_{\mathrm{m}}^{+}=\delta_{\mathrm{n}, \mathrm{m}}
$$

The modal expansion (2.26) is similar to that used by Randall and St. John.

One mode is now concentrated on the first overtone $\psi_{1}$, assuming the oscillations to be primarily in this mode. Noting that in one-group theory the eigenfunctions $\psi_{\mathrm{n}}$ are self-adjoint, equations (2.21) and (2.22) are multiplied by $\psi_{1}$ and integrated over the core volume. The resulting equations are written in the form: 


$$
\begin{gathered}
\frac{\partial\langle\mathrm{I}\rangle}{\partial t}=\left\langle\beta_{i}\right\rangle\langle\delta \phi\rangle-\lambda_{i}\langle\mathrm{I}\rangle \\
\frac{\partial\langle\mathrm{X}\rangle}{\partial t}=\lambda_{i}\langle\mathrm{I}\rangle+\left\langle\beta_{\mathrm{X}}\right\rangle\langle\delta \phi\rangle-\langle\gamma\rangle\langle\delta \phi\rangle-\langle\mathrm{A}\rangle\langle\mathrm{X}\rangle
\end{gathered}
$$

where

$$
\begin{aligned}
& \langle I\rangle=\int d \underline{r} \delta N_{i}(\underline{r}, t) \psi_{1}(\underline{r}) \\
& \langle X\rangle=\int d \underline{r} \delta N_{x}(r, t) \psi_{1}(\underline{r}) \\
& \langle\delta \phi\rangle=\int \mathrm{d} \underline{\mathrm{r}} \delta \phi(\underline{\mathrm{r}}, \mathrm{t}) \psi_{1}(\underline{\mathrm{r}}) \\
& \left\langle\beta_{i}\right\rangle=\frac{\int d \underline{r} y_{i} \sum_{\underline{f}}(\underline{r}) \delta \phi(\underline{r}, t) \psi_{1}(\underline{r})}{\int d \underline{r} \delta \phi(\underline{r}, t) \psi_{1}(\underline{r})} \\
& \left\langle\beta_{x}>=\frac{\int d \underline{r}_{x} \sum_{\underline{f}}(\underline{r}) \delta \phi(\underline{r}, t) \psi_{1}(\underline{r})}{\int d \underline{r} \delta \phi(\underline{r}, t) \psi_{1}(\underline{r})}\right. \\
& \left\langle\gamma_{X}\right\rangle=\frac{\int d \underline{r} N_{x_{0}}(\underline{r}) \sigma_{x} \delta \phi(\underline{r}, t) \psi_{1}(\underline{r})}{\int d \underline{r} \delta \phi(\underline{r}, t) \psi_{1}(\underline{r})} \\
& \langle A\rangle=\frac{\int d \underline{r}\left[\sigma_{x} \phi_{0}(\underline{r})+\lambda_{x}\right] \delta N_{x}(\underline{r}, t) \psi_{I}(\underline{r})}{\int d \underline{r} \delta N_{x}(\underline{r}, t) \psi_{I}(\underline{r})}
\end{aligned}
$$


For flux variations in the first overtone only, and with the assumption that the variations in the iodine and xenon concentrations are in that same mode, the coefficients $\left\langle\beta_{i}\right\rangle,\left\langle\beta_{x}\right\rangle,\left\langle\gamma_{x}>\right.$ and $\langle A\rangle$ in equations (2.29) and (2.30) are time-independent. In a digital simulation of this mode of oscillation, equations (2.29) and (2.30) would be solved by assuming $\langle\delta \phi\rangle$ to be constant in time during a time-step length $\tau$. Equations (2.29) and (2.30) can then be solved exactly, with solutions

$$
\begin{gathered}
\left.\langle I\rangle\right|_{t+\tau}=\left.a_{11}\langle I\rangle\right|_{t}+\left.a_{13}\langle\delta \phi\rangle\right|_{t} \\
\left\langle\left. X\right|_{t+\tau}=a_{22}\left\langle\left. X\right|_{t}+\left.a_{21}\langle I\rangle\right|_{t}+\left.a_{23}\langle\delta \phi\rangle\right|_{t}\right.\right.
\end{gathered}
$$

where

$$
\begin{gathered}
a_{11}=e^{-\lambda_{i} \tau} \\
a_{13}=\frac{\left(1-e^{-\lambda_{i} \tau}\right)}{\lambda_{i}<\beta_{i}>} \\
a_{21}=\frac{a_{i}\left(e^{-\lambda_{i} \tau}-e^{-<A>\tau}\right)}{<A>-\lambda_{i}} \\
a_{23}=\left(<\beta_{i}>+\left\langle\beta_{x}>-\left\langle\gamma_{x}>\right) \frac{\left(1-e^{-<A>\tau}\right)}{<A>}-\left\langle\beta_{i}>\frac{\left(e^{-\lambda_{i} \tau}-e^{-<A>\tau}\right)}{<A>-\lambda_{i}}\right.\right.\right.
\end{gathered}
$$

Since the oscillations being considered are in the mode $\psi_{1}, \delta N_{i}(\underline{r}, t)$ and $\delta N_{X}(\underline{r}, t)$ behave as $e^{\omega t} \psi_{1}(\underline{r})$. Thus, equations $(2.31)$ and $(2.32)$, 


$$
\begin{aligned}
& \left.\langle I\rangle\right|_{t+\tau}=e^{\omega \tau}<I>\left.\right|_{t} \\
& \left\langle\left. X\right|_{t+\tau}=e^{\omega \tau}<X>\left.\right|_{t}\right.
\end{aligned}
$$

Substitution of equations (2.41) and (2.42) into equations (2.38) and (2.39) yields the equations

$$
\begin{gathered}
\left.\left(a_{11}-e^{\omega \tau}\right)<I\right\rangle+a_{13}\langle\delta \phi\rangle=0 \\
\left(a_{22}-e^{\omega \tau}\right)<X>+a_{21}\langle I\rangle+a_{23}\langle\delta \phi\rangle=0
\end{gathered}
$$

where the weighted quantities are evaluated at time $t$. Quantity <I> can be eliminated from equations (2.43) and (2.44), and therefore

$$
\langle\mathrm{X}\rangle=\mathrm{C}\langle\delta \phi\rangle
$$

where

$$
C=\frac{\left(\frac{a_{12} a_{13}}{a_{11}-e^{\omega \tau}}\right)-a_{23}}{a_{22}-e^{\omega \tau}}
$$

Now in equation (2.45), $\langle X\rangle$ and $\langle\delta \phi\rangle$ are the integrated weighted excess xenon and flux distributions at time $t$, and $C$ must therefore be independent of the time-step length $\tau$. That this is so can be seen by multiplying the equation (2.20) for the excess flux by $\psi_{1}(\underline{r})$ and integrating over the core volume. From equation (2.46), $\omega$ is clearly a function of the time-step length $\tau$. If the limit of equation (2.46) is taken as $\tau \rightarrow 0$, and $\omega_{0}$ is defined equal to $\omega$ as $\tau \rightarrow 0$, after using equations (2.40), equation (2.46) simplifies to:

$$
C=\frac{\left\langle\beta_{i}\right\rangle \lambda_{i}-\left(\left\langle\gamma_{x}\right\rangle-\left\langle\beta_{x}\right\rangle\right)\left(\lambda_{i}+\omega_{0}\right)}{\left(<A>+\omega_{0}\right)\left(\lambda_{i}+\omega_{0}\right)}
$$


By eliminating $C$ from equations (2.46) and (2.47), a relationship can be obtained between $\omega, \tau$ and $\omega_{\circ}$. Equations (2.46) and (2.47) can be written in the following quadratic form:

$$
\begin{aligned}
& e^{2 \omega \tau}+b e^{\omega \tau}+c=0 \\
& \omega_{0}^{2}+b^{\prime} \omega_{0}+c^{\prime}=0
\end{aligned}
$$

Note that $\omega$ and $\omega_{0}$ are complex numbers, with the real part equal to the stability index as defined in Appendix B. The roots of equations (2.48) and (2.49) are, respectively:

$$
\begin{aligned}
& e^{\omega \tau}=-\frac{b}{2} \pm i \sqrt{\frac{4 c-b^{2}}{4}} \\
& \omega_{0}=-b^{\prime} \pm i \sqrt{\frac{4 c^{\prime}-b^{\prime 2}}{4}}
\end{aligned}
$$

where the square roots in the above equations are real quantities.

Noting that for $z$ a complex number $(z=x+i y),\left|e^{z}\right|^{2}=e^{2 x}$, equations (2.50) and (2.51) yield

$$
\begin{aligned}
& e^{2 \tau R(\omega)}=c \\
& R\left(\omega_{0}\right)=-\frac{b}{2}^{\prime}
\end{aligned}
$$

where $R(z)$ is the real part of $z$.

Substituting the expressions for $c$ and $b^{\prime}$ in equations (2.52) and (2.53), eliminating $C$ between the equations, and using equation (2.40) one obtains: 


$$
\begin{gathered}
2 \tau R(\omega)=e^{-\left(\lambda_{i}+\langle A>) \tau\right.} \\
+\left[2 R\left(\omega_{0}\right)+\langle A\rangle+\lambda_{i}\right]\left[\frac{\left[\beta_{i}>\left(e^{-\lambda \tau i}-e^{-<A>\tau}\right)\right.}{<A>-\lambda_{i}}\right. \\
\left.-\frac{\left(\left\langle\beta_{i}\right\rangle+\left\langle\beta_{x}\right\rangle-\left\langle\gamma_{x}\right\rangle\right)\left(e^{-\left(\lambda_{i} \tau\right)}-e^{-\left(\lambda_{i}+\langle A>) \tau\right.}\right)}{\langle A\rangle}\right]\left[\left\langle\gamma_{x}>-\left\langle\beta_{x}>\right]-1\right.\right.
\end{gathered}
$$

Equation (2.54) is similar to equation (4.8) in reference 13, except that weighted average quantities appear in equation (2.54), while in arriving at this equation Davison assumed that the coefficients in equations (2.21) and (2.22) were independent of space. By using a perturbation argument, Davison has shown [13] that, in the case where the quantity $\sigma_{x} \phi_{0}$ in equation (2.22) was allowed to depend on position, while retaining the assumption that $\mathrm{y}_{i} \Sigma_{f}, \mathrm{y}_{\mathrm{x}} \Sigma_{f}$ and $\mathrm{N}_{\mathrm{x}_{0}} \sigma_{\mathrm{x}}$ were constant in space, his equation (4.8) was still valid if an average weighted value of $\sigma_{x} \phi_{0}$ was used in the equation. The derivation above, however, shows that for the particular mode of oscillation considered, equation (2.54) is valid for arbitrary spatial dependence of the above quantities, provided properly weighted average quantities are used. Davison [13] has shown that equation (2.54) can be further simplified if the following conditions hold:

$$
\begin{gathered}
\frac{-\lambda_{i}}{\langle A>1} \ll 1 \\
\lambda_{i} \tau \ll 1 \\
|\tau R(\omega)| \ll 1
\end{gathered}
$$


With the assumptions (2.55), equation (2.54) becomes

$$
R(\omega)=R\left(\omega_{0}\right) \frac{\left(1-e^{-<A>\tau}\right)}{<A>\tau}-\frac{\lambda_{1}}{2}\left[1+e^{-<A>\tau}-\frac{2\left(1-e^{-<A>\tau}\right)}{<A>\tau}\right]
$$

Equation (2.56) is in a very useful form, since the observed $R(\omega)$ is related to the true, or extrapolated $R\left(\omega_{0}\right)$ through the dimensionless variable $\langle A\rangle \tau$. Equation (2.56) can therefore be thought of as a universal relationship between $R(\omega)$ and $R\left(\omega_{0}\right)$ in terms of a reduced time-step length $\tau_{r}=\langle A\rangle \tau$. Note that in the more exact equation (2.54), $R(\omega)$ and $R\left(\omega_{0}\right)$ are related not only through the reduced time-step length ${ }^{\tau}{ }_{r}$, but also through the actual time-step length $\tau$ and the quantities $\left\langle\beta_{i}\right\rangle,\left\langle\beta_{x}\right\rangle,\left\langle\gamma_{x}\right\rangle$, and $\langle A\rangle$.

Figure 2-4 compares equations (2.54) and (2.56), where typical parameters for a large PWR were used in equation (2.54). In using equation (2.54), the weighted average quantities were approximated by volume average values. As shown below, this is a good approximation when the oscillation is restricted to the first overtone. It appears from Figure 2-4 that the approximation (2.56) to equation (2.54) is not particularly good for a typical PWR design. However, the suggestion of Barss [9] has been followed and equation (2.56) was used as a correlation between $R(\omega)$ and $R\left(\omega_{0}\right)$, with $\langle A\rangle$ a fitting parameter. This approach is justified on two grounds. First, it appears from Figure 2-4 that equation (2.56) can be a good approximation to equation (2.54) if an effective value of $\langle A\rangle$ is used in the equation. Second, in view of the approximations made in arriving at equation (2.54) or (2.56), these equations, when applied to an actual digital simulation of xenon oscillations, may be expected to yield a good qualitative description of the behavior of the solutions. However, they should not be expected to yield accurate quantitative results. Thus, the use of $\langle A\rangle$ as a fitting parameter seems indicated.

\subsubsection{Development of a Correlation for the Reduced Time-Step Length $\langle A\rangle{ }^{\prime} \tau$}

A series of numerical calculations was performed with program ZEST in order to develop a correlation for the reduced time-step length $\tau_{r}$, or equivalently, the quantity $\langle A>$ in equation (2.56). It is useful, however, to study the 


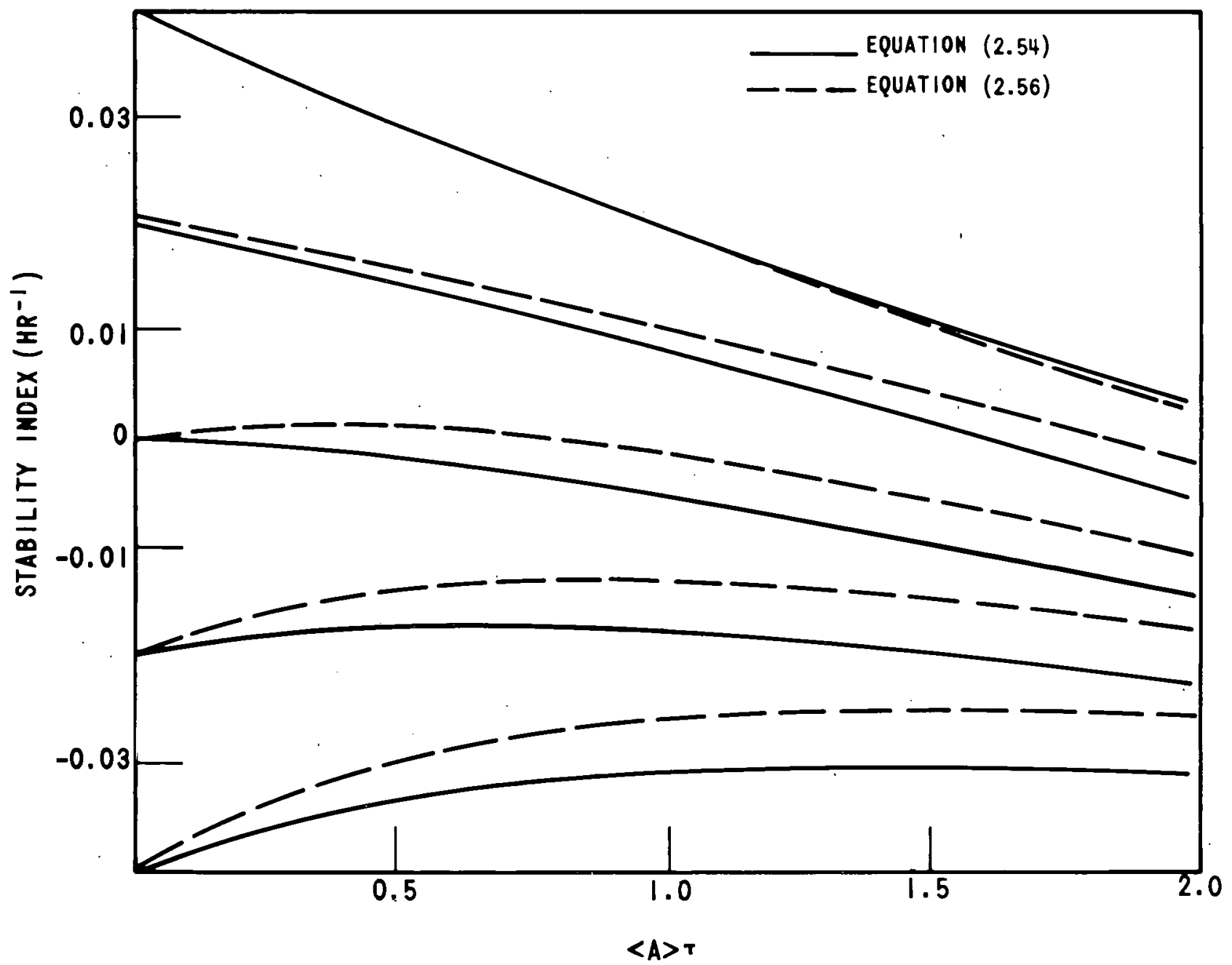

Figure 2-4. Comparison of Exact and Approximate Correlations of Stability Index as a Function of Time-Step Length 
definition of <A> given in equation (2.37) in the derivation of equation (2.56). For oscillations in the first overtone, if one assumes $\delta \mathrm{N}_{x}(\underline{r}, t)$ to be proportional to $e^{\omega t} \psi_{1}(\underline{r})$, then equation (2.37) reduces to

$$
\langle A\rangle=\sigma_{x} \frac{\int d \underline{r} \phi_{0}(\underline{r}) \psi_{1}^{2}(\underline{r})}{\int d \underline{r} \psi_{1}^{2}(\underline{r})}+\lambda_{x}
$$

Now, if the approximation is made

$$
\frac{\int d \underline{r} \phi_{0} \underline{(r)} \psi_{1}^{2}(\underline{r})}{\int d \underline{r} \psi_{1}^{2}(\underline{r})} \simeq \frac{\int d \underline{r} \phi_{0}(\underline{r})}{\int d \underline{r}}=\bar{\phi}_{0}
$$

then equation (2.57) can be written

$$
<A>=\sigma_{x} \bar{\phi}_{0}+\lambda_{x}
$$

Equation (2.58) is exact for a flat flux, while for a cosine distribution with flat fluxes in the other dimensions, and with $\psi_{1}$ the first geometric overtone, the error in equation (2.58) is less than 7 percent. From equation (2.59), it is evident that a correlation for $\angle A>$ of the form

$$
<A>=a \bar{\phi}_{0}+b
$$

where $a$ and $b$ are fitted parameters, should be sought for. In addition to the appealing simplicity of the correlation equation (2.60), it is physically intuitive that the error introduced by a finite time-step length should be a function primarily of the flux level, and only secondarily of flux distribution, core size, etc., since it is the assumption of a constant flux level in equations (2.21) and (2.22) for the duration of tne time-step which introduces an error in the solution. 
In addition to the calculations shown in Figure 2-1 for the standard 11-foot core with a flattened power distribution and fuel temperature feedback, two additional cores were studied which had significantly different characteristics from that of Figure 2-1. The results of the calculations for time-step lengths of one, two, and four hours are shown in Figures 2-5 and 2-6. Both sets of calculations are for a 10-foot core, 2.6 a/o enriched, with uniform loading and no temperature feedback. Figure 2-5 corresponds to an average power density of $84.5 \mathrm{w} / \mathrm{cc}\left(\bar{\phi}_{0}=4.5 \times 10^{13}\right)$, and Figure 2-6 corresponds to an average power density of $106 \mathrm{w} / \mathrm{cc}\left(\bar{\phi}_{0}=5.6 \times 10^{13}\right)$. The former core is stable while the latter is unstable.

Figure $2-7$ is a plot of equation (2.56). The results of the numerical calculations shown on Figures $2-1,2-5$ and 2-6 were graphically fitted to equation (2.56), with the best fits shown in Figure 2-7. In arriving at these results, the stability index used throughout was the one defined in Appendix B.

It can be seen from Figure 2-7 that the numerical results can be very well fitted by an expression of the form (2.56). Moreover, it is important to realize that the 11-foot core and one of the 10-foot cores analyzed were both operated at the same average power density of $84.5 \mathrm{w} / \mathrm{cc}$. For both of these cases, the best fit to equation (2.56) yields the same value for the parameter $\langle A\rangle$, as can be observed from Figure 2-7. The second 10-foot core was operated at $106 \mathrm{w} / \mathrm{cc}$. Therefore it appears, based on the above calculations, that $\langle A\rangle$ can be well represented by an expression of the form (2.60). From the data of Figure $2-7,\langle A\rangle$ is found, using a least squares fit, to be

$$
<A>0.0560+0.0733 \times 10^{-13} \bar{\phi}_{0}
$$

This expression for $\angle A>$ is shown graphically in Figure 2-8, where it is compared to the similar expression for $<A>$ given by the exact formulation of equation (2.59). As can be seen there the agreement is reasonably good, considering the approximations assumed in: a) the basic theory; b) the derivation of $\langle A\rangle$ (refer to Figure 2-4); c) graphically fitting the points on Figure 2-7. This agreement must imply that $\langle A\rangle$ is insensitive 


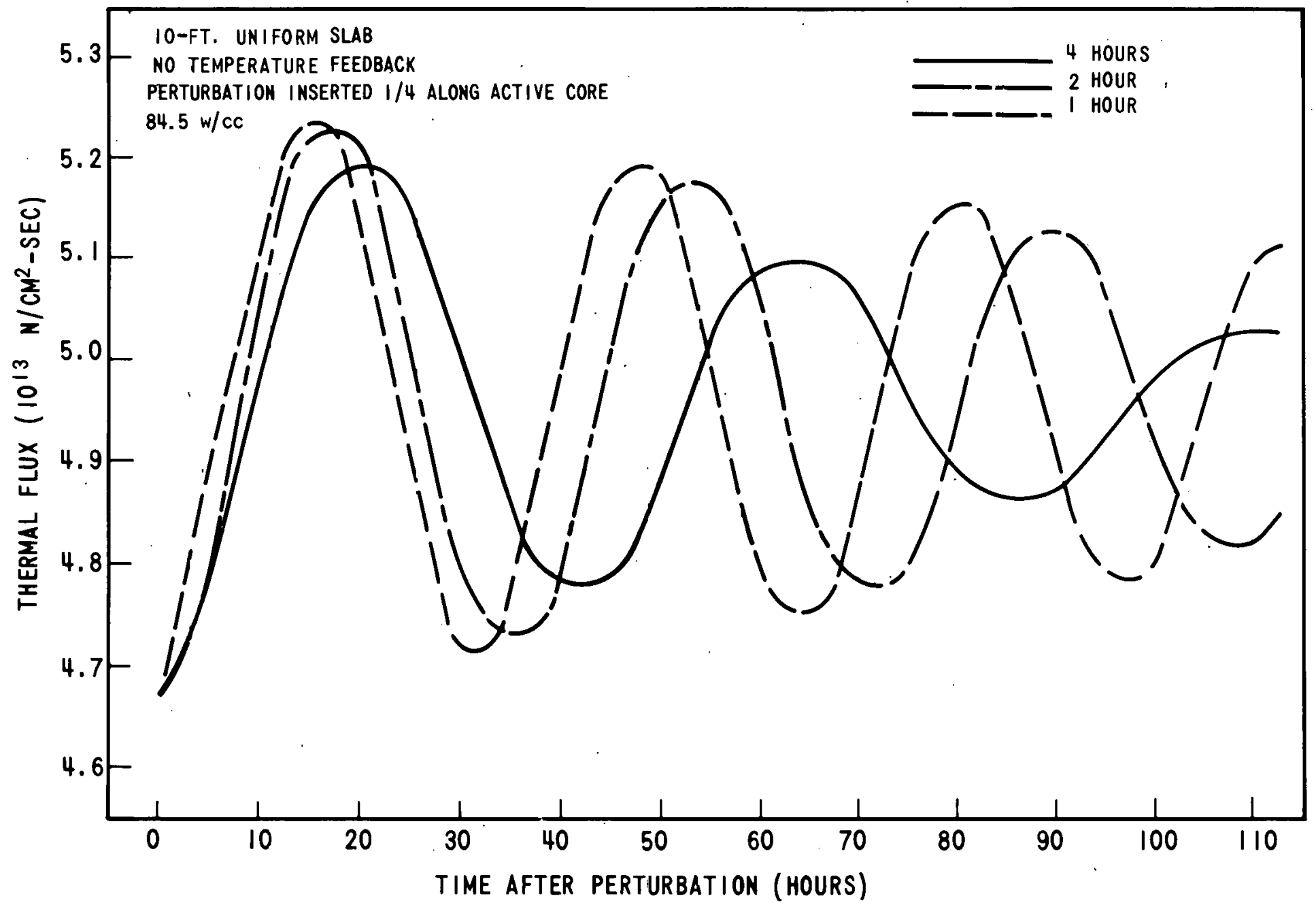

Figure 2-5. Variation of Xenon-Induced Spatial Stability Characteristics with Time-Step Length in Digital Calculations ( $84.5 \mathrm{w} / \mathrm{cc})$ 


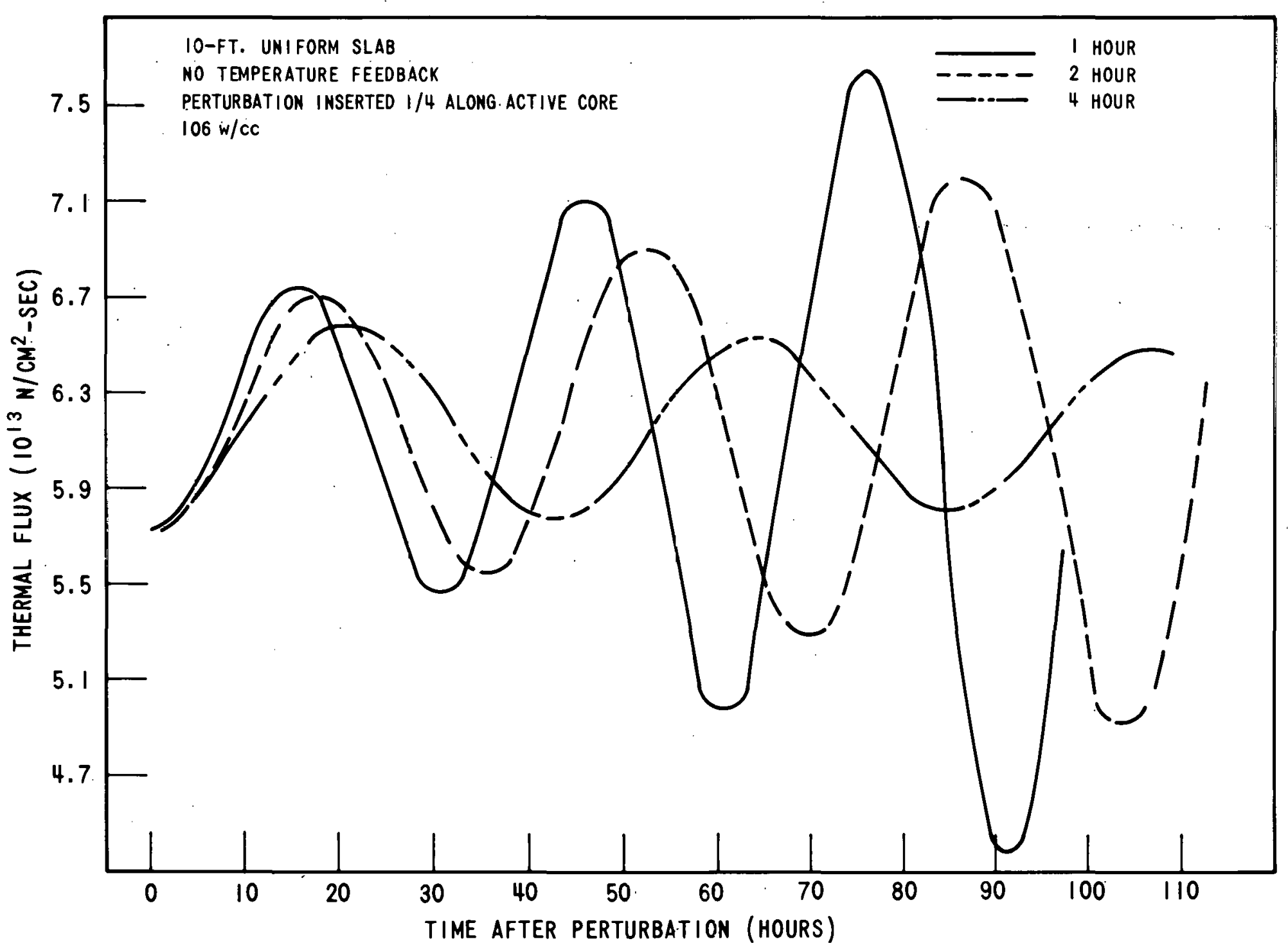

Figure 2-6. Variation of Xenon-Induced Spatial Stability Characteristics with Time-Step Length in Digital Calculations (106 w/cc) 


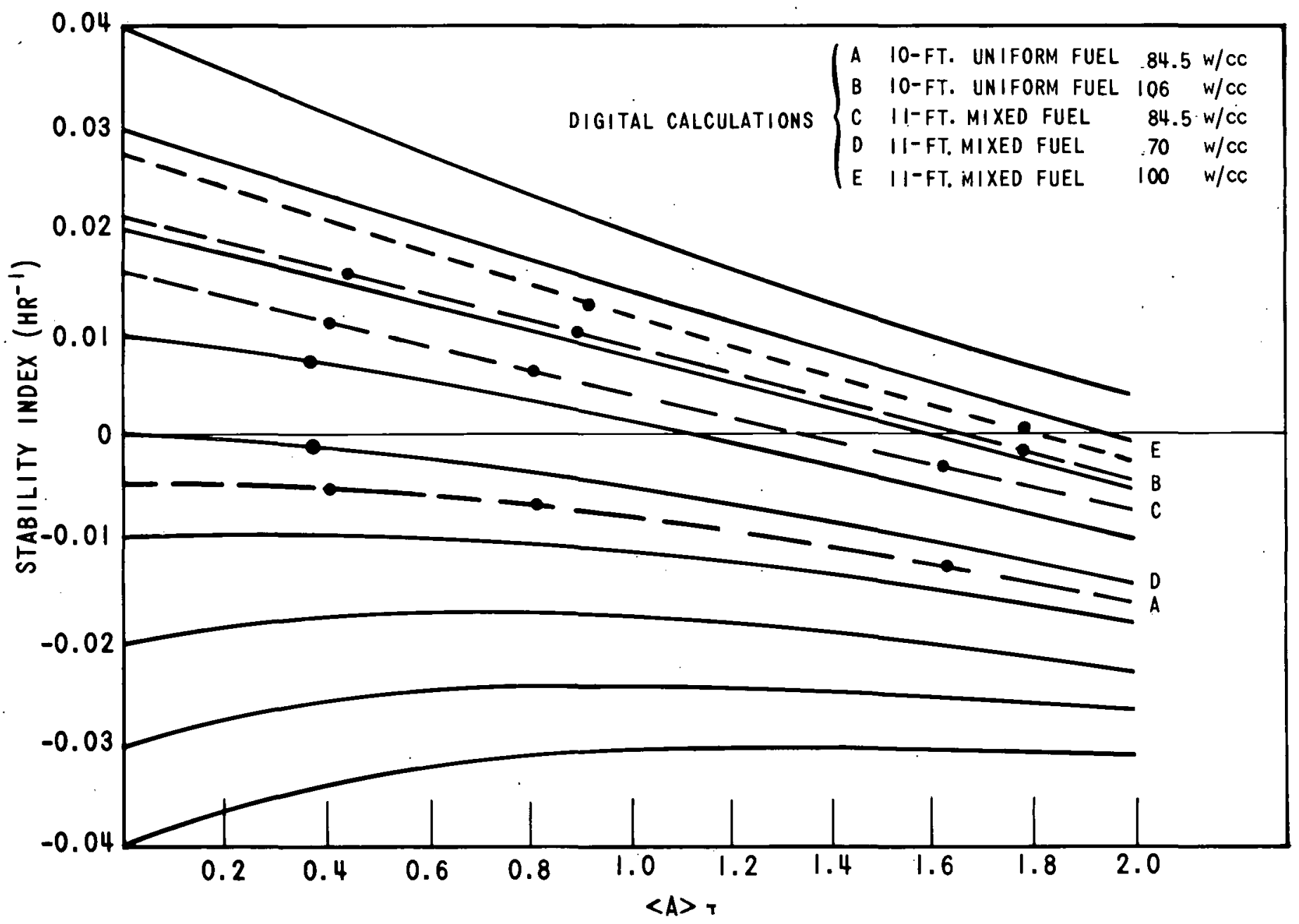

Figure 2-7. Comparison of Correlation from Equation (2.56) and Digital Calculations 


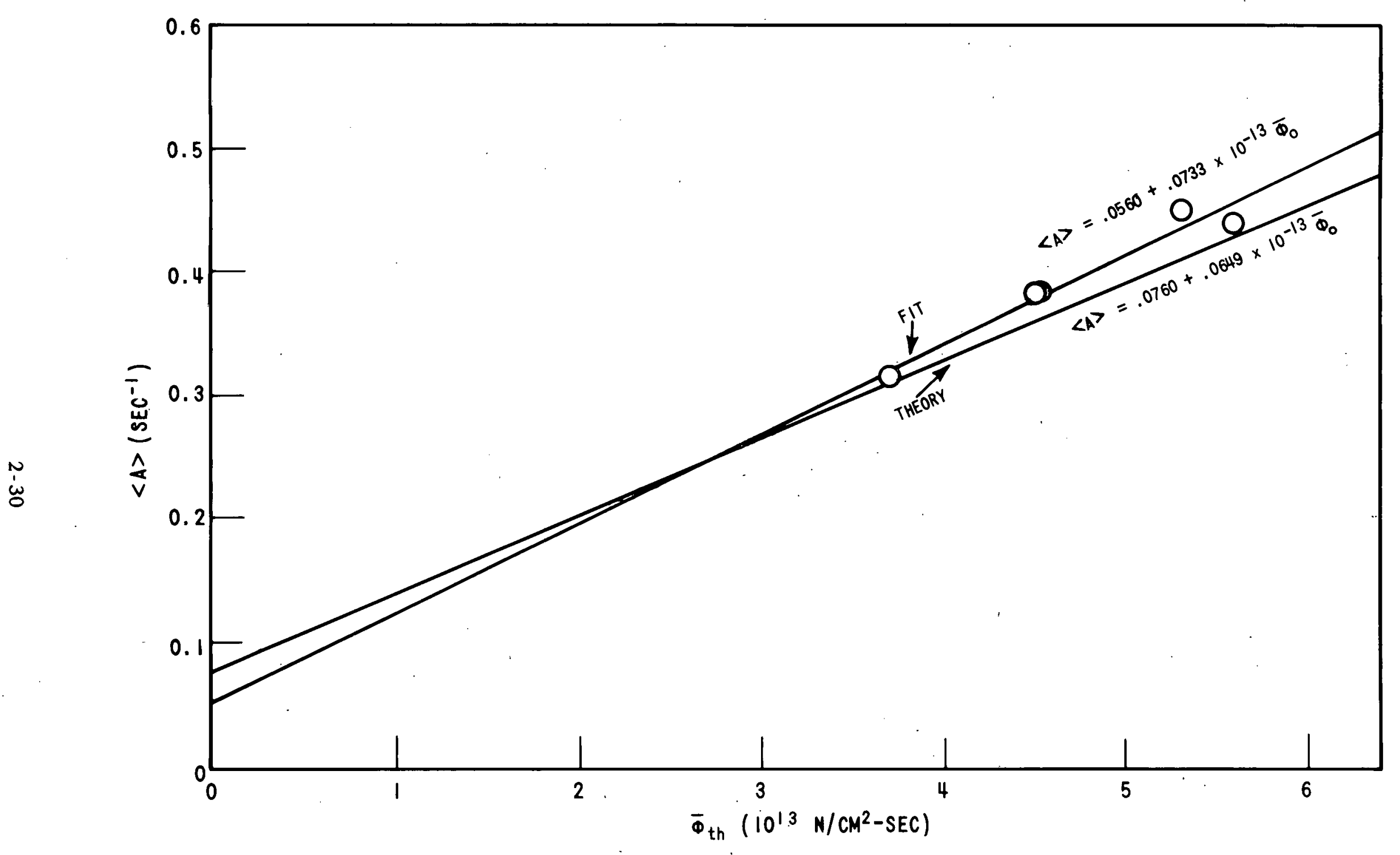

Figure 2-8. Comparison of Theoretical and Calculated Value of $\langle A\rangle$ as a Function of Power Level 
to almost all core parameters except $\bar{\phi}_{0}$. In terms of the average power density, $p$, for an average enrichment of $2.6 \mathrm{a} / \mathrm{o},\langle\mathrm{A}\rangle$ is given by

$$
<A>=0.0560+0.0039 \mathrm{P}
$$

where $p$ is in units of $\mathrm{w} / \mathrm{cc}$.

Using the above correlátion for $\langle A\rangle$, Figure 2-9 was generated, showing the variation of apparent (observed) stability index as a function of time-step length, for an average power density of $84.5 \mathrm{w} / \mathrm{cc}$. For different power densities, Figure 2-7 should be used together with equations (2.61) or (2.62). It is worthwhile to note the functional behavior shown on Figure 2-9. In general, a core which is unstable to the first overtone will be predicted to be relatively more stable by digital calculations with a non-zero time-step length. The error in the observed stability index introduced by the finite time-step length increases as the stability index becomes more positive. There is a region in the stability plane, for stability indices in the neighborhood of -0.02 , where the results are relatively insensitive to the length of the time-step. For large and negative stability indices (very stable cores), the use of a finite time-step length leads to a conservative estimate of stability. Finally, the error introduced by the use of non-zero time-step length becomes relatively larger as the average flux level is increased.

Appendix $C$ shows some numerical results from ZEST in which, among other things, the time-step corrected stability index is shown. A number of these results show that, for a variety of time-step lengths, applying the correction factor produces very good agreement between the resulting stability indices. This can be seen by examining cases 1 through 13.

\subsubsection{Effect of Finite Flux Convergence Criterion}

As in the numerical solution of all initial value problems, the cumulative error introduced both by the finite difference scheme and the convergence criteria can become very important. As just noted in the previous paragraphs, the discussion on time-step length comes under the former category, while in the present discussion the spatial flux convergence comes under the latter. 


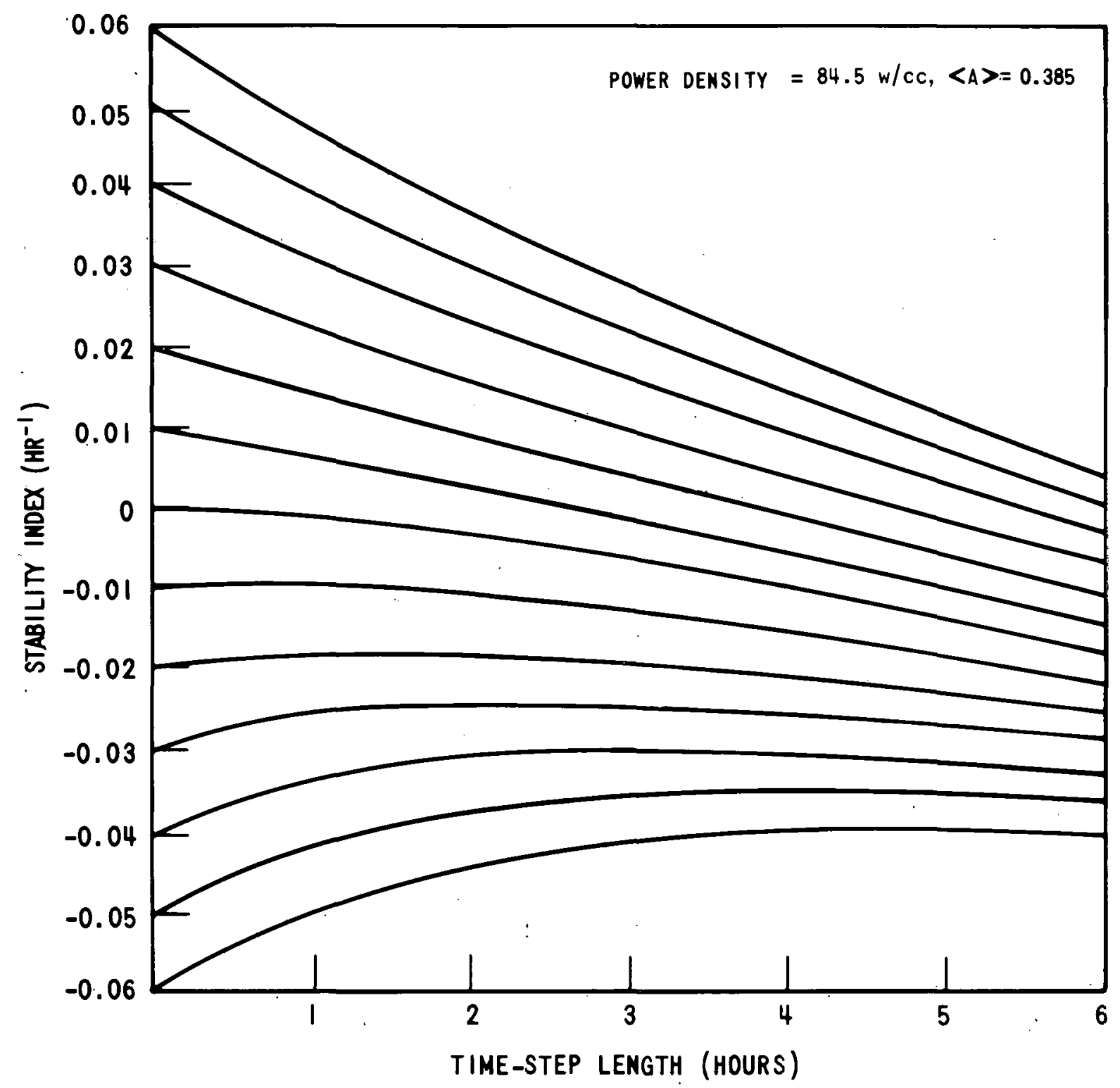

Figure 2-9. Effect of Time-Step Length on Spatial Stability Index 
Since the source guess for each new time-step is its previous converged value, the source should converge from below while the power is increasing, and should converge from above when the flux is decreasing provided the source at each iteration does not oscillate about the converged value. . This was indeed found to be the case both for the normal iteration procedure and the iteration procedure with an extrapolated source (See Appendix D). Thus, the error introduced by the finite convergence criterion is by no means random and could actually constitute a problem.

From the above qualitative argument it would appear that divergent oscillations will be suppressed when too large a convergence condition is applied. Similarly, convergent oscillations will be suppressed. While the effect should be in the same direction as the finite time-step effect when oscillations are divergent, the effect should be in the opposite direction for strongly convergent oscillations.

The results are shown in Appendix C, Cases 31 through 33, where various values of convergence criterion are compared with the corresponding stability indices. It can be seen that the time-step-corrected stability index is indeed quite sensitive to the convergence criterion and, as predicted, it is in a direction such that a finite convergence criterion underestimates the true stability index.

A possible method of suppressing this effect will be discussed in Section 2.3.8, Modified Procedures.

\subsubsection{Effect of a Finite Spatial Mesh Size}

Diffusion-depletion calculations of spatial oscillations have the one advantage that realistic core configurations and perturbations can be analyzed. They also provide space-time flux responses that can be used directly in developing control procedures. Multidimensional calculations are, however, costly, since a relatively large number of time-steps and a tight convergence criterion are required for each calculation. In two-dimensional $x-y$ geometry, half-core or full-core calculations are required to adequately treat azimuthal oscillations. 
To maintain computing time within reasonable limits, it is often necessary to use a coarse spatial mesh description of the core. In both ZEST and TURBO*, the mesh length was varied to determine the sensitivity of the stability characteristics to this parameter. Using ZEST, a 10-foot core without any temperature feedback and with uniform fuel loading, and an 11-foot core with fuel temperature feedback and flattened power distribution were examined for senstivity to mesh length variation. The 10-foot core was investigated with mesh lengths of 2.5 and $5 \mathrm{~cm}$, and the 11-foot core with $2 \mathrm{~cm}$ and $4 \mathrm{~cm}$. For the two-dimensional TURBO* calculations, a $10.8 \mathrm{~cm}$ spatial mesh was chosen for an 11-foot diameter core and the results were compared with those of a core differing only in that it had a $5.4 \mathrm{~cm}$ mesh. Although comparable running times for the ZEST calculations could not be evaluated, those for TURBO* appeared to show that running time was quite sensitive to mesh length. For the two TURBO* cases examined, it appears that by doubling the mesh length, running time is reduced by approximately a factor of four. As in the ZEST cases examined, this was achieved with relatively little loss of computational accuracy; i.e., space-time behavior of the gross flux distribution is well represented by a coarse mesh.

Figure 2-10 compares the ratio of assembly average power to core average power following the introduction of a perturbation in TURBO* for the $10.8 \mathrm{~cm}$ and $5.4 \mathrm{~cm}$ meshes. The figure shows this ratio for two assemblies symmetrically located along a diameter $\left(0^{\circ}\right.$ and $\left.180^{\circ}\right)$. The core selected for comparison has an initial power distribution which is dished toward the center of the core and has an average enrichment of 2.5 percent, resulting in relatively large oscillations. The use of the relatively coarser mesh tends to overestimate the power peak by a few percent, with only a negligible effect on the period of oscillation.

Figure 2-11 shows the steady-state power distributions and the power distributions eight hours following the perturbation, taken along the axis $0^{\circ}-180^{\circ}$. Calculations using both spatial mesh widths predict similar oscillation characteristics, with the relatively coarser mesh tending to overestimate slightly the peak in one side of the core, and to underestimate the depression in the other side of the core. 


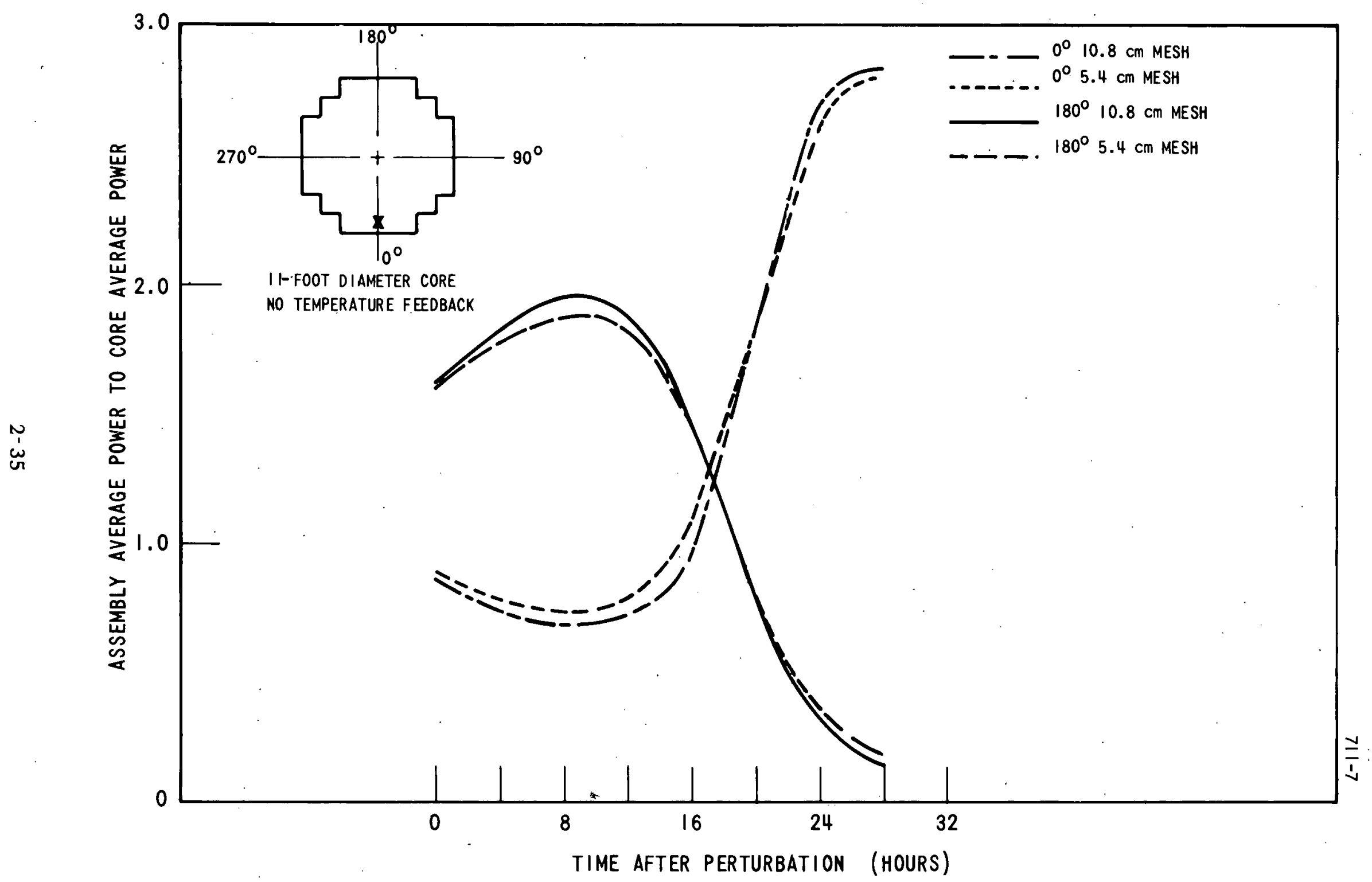

Figure 2-10. Effect of Spatial Mesh Size on Xenon-Induced Oscillations 


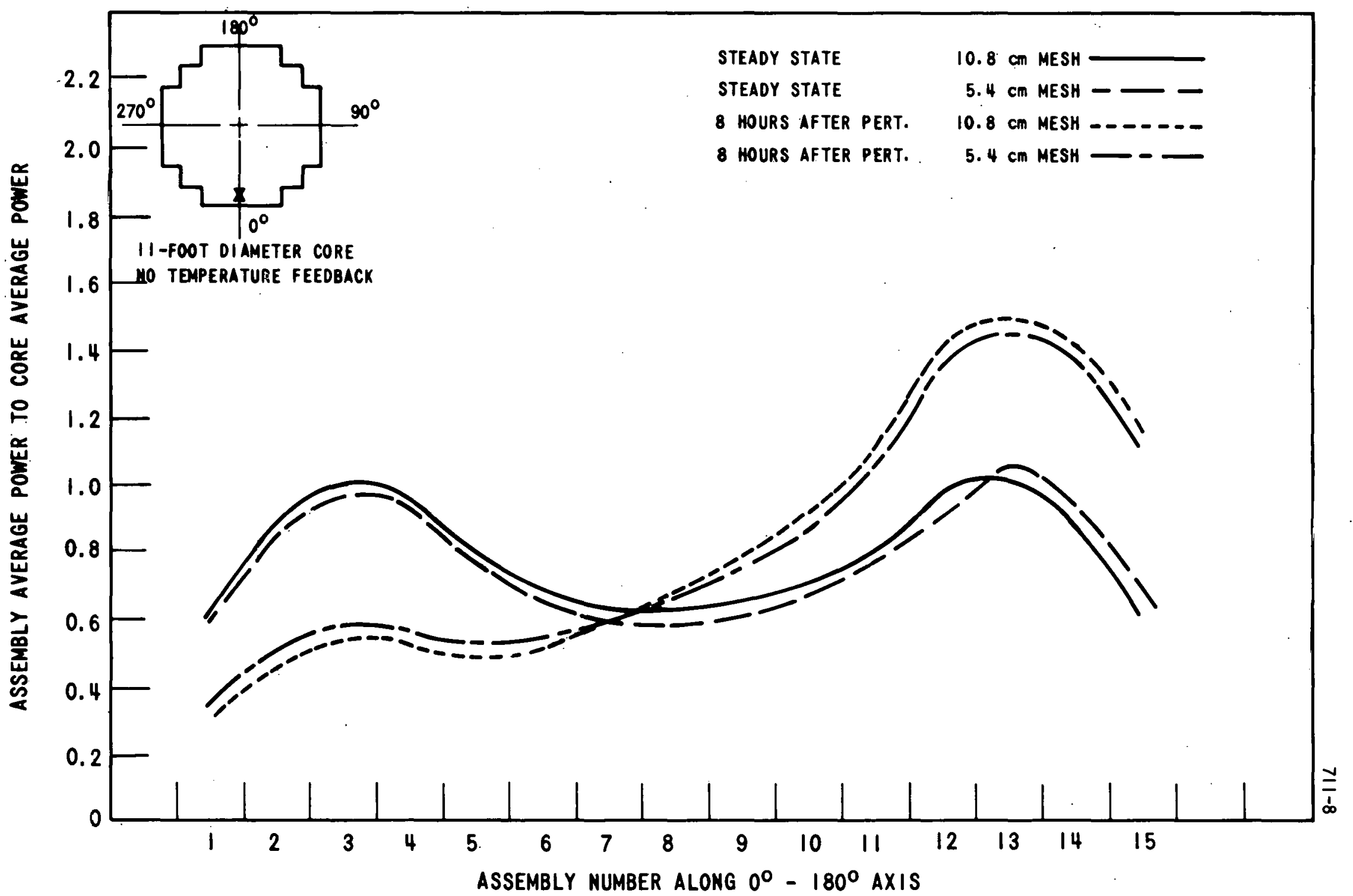

Figure 2-11. Effect of Spatial Mesh Size on Power Distribution 
The results of a similar mesh study using ZEST can be seen in Appendix C, Cases 34 through 37. Although these results tend to support the TURBO* calculations in that a fairly coarse mesh is justified, the effect of mesh size on the stability index for the two codes is in disagreement because TURBO* predicts an increase in the stability index for increased mesh size, while ZEST predicts a decrease. This difference, however, is slight and the effect could be due to the different numerical schemes used in the two codes.

\subsubsection{Effect of a Finite Energy Mesh}

The use of two energy groups in the calculation of spatial xenon instabilities in large PWR's assumes tight energy coupling at all points. Some idea as to the validity of this assumption can be obtained by analyzing the fast and thermal space-dependent transfer functions with xenon feedback. The theory of space-dependent transfer functions and the program SPARTA are briefly discussed in Section 2.5.

The space-dependent transfer function was calculated with SPARTA across a one-dimensional reflected slab which simulated diametral oscillations. An 11-foot core size was employed, and zone enrichments were used to obtain a flattened flux distribution. The fundamental mode was suppressed in the calculations. Energy coupling can be evaluated by comparing the fast and thermal decibel ( $\mathrm{db}$ ) gain and phase shift at the resonant frequency correspondIng to the first overtone. For total energy coupling, the $\mathrm{db}$ gain and phase shift will be identical in the fast and thermal energy groups for all space points. Table 2-1 lists results of calculations for a core with different temperature feedback. The $d b$ gains and phase shifts are taken at a space point corresponding to the peak of the first overtone flux shape. Three cases in Table 2-1 are unstable in the flrst overtone.

It is noted that the difference between the fast and thermal transfer functions is small. The fast-thermal differences given in Table 2-1 were approximately constant across the core. With temperature feedback, the fast-thermal difference is relatively larger than with no temperature feedback. This is probably due to the effect of temperature on resonance absorptions, which is a fast group effect, while the absorption in Xe-135 
TABLE 2-1

COMPARISON OF THE FAST AND THERMAL SPACE-DEPENDENT TRANSFER FUNCTIONS

\begin{tabular}{|c|c|c|c|c|c|c|}
\hline Case & $\begin{array}{c}\text { Temperature } \\
\text { Feedback }\end{array}$ & $\begin{array}{c}\text { First Overtone } \\
\text { Frequency } \\
\text { (cps) }\end{array}$ & $\begin{array}{c}\text { Fast } \\
\text { db Gain }\end{array}$ & $\begin{array}{c}\text { Thermal } \\
\text { db Gain }\end{array}$ & $\begin{array}{c}\text { Fast-Thermal } \\
\text { db Gain } \\
\text { Difference }\end{array}$ & $\begin{array}{c}\text { Fast-Thermal } \\
\text { Phase Shift } \\
\text { Difference } \\
\left({ }^{\circ}\right)\end{array}$ \\
\hline 1 & $\begin{array}{c}\text { None } \\
\text { Fuel } \\
\text { Temperature } \\
\text { Fuel } \\
\text { Temperature } \\
\text { Moderator } \\
\text { Density } \\
\left(0.6 \times 10^{-4} /{ }^{\circ} \mathrm{F}\right)\end{array}$ & $6.6 \times 10^{-6}$ & 16.528 & 16.653 & -0.125 & 0.125 \\
& $5.6 \times 10^{-6}$ & 13.159 & 13.304 & -0.161 & 0.282 \\
\hline
\end{tabular}

is primarily a thermal effect. It should also be noted that in a large PWR, the effect of the reflector is very small, i.e., its presence has little effect on the fast and thermal space-dependent transfer functions in the vicinity of the reflector.

From this brief discussion, it is concluded that the use of two energy groups -- and even one energy group -- is probably adequate for xenon spatial stability studies in large PWR's.

\subsubsection{Treatment of Feedback Effects}

\subsubsection{Non-linear Feedback Effects}

The power defect in a PWR is a marked non-linear function of power. [14] This behavior is primarily a result of the non-linear variation of fuel temperature with power density as a result of the heat transfer characteristics of the fuel rod. The sensitivity of the spatial stability characteristics to this non-linear feedback behavior is briefly investigated here. 
As was noted in Section 2.3.1, the fuel temperature feedback effects in ZEST are accounted for through the use of polynominal representation of fuel temperature versus power density, and fast absorption and removal cross section versus temperature:

$$
\begin{aligned}
& T_{f}=a_{0}+a_{1} P+A_{2} P^{2} \\
& \Sigma_{a_{1}}=b_{0}+b_{1} T_{f}+b_{2} T_{f}^{2} \\
& \Sigma_{R}=c_{0}+c_{1} T_{f}+c_{2} T_{f}^{2}
\end{aligned}
$$

where the $a^{\prime} s, b^{\prime} s$, and $c^{\prime}$ s are regionwise input contents. Equation (2.63) represents the local heat transfer process, while equations (2.64) and (2.65) represent the Doppler effect on resonance absorption. The importance of accounting for the non-linear behavior of the fuel temperature feedback effect in digital simulation was investigated by comparing ZEST calculations with the full quadratic treatment of equations (2.63) to (2.65) with calculations where either the heat transfer or the Doppler effect equations were linearized. In linearizing equations (2.63) to (2.65), the coefficient of the linear term was specified so as to correspond to the slope obtained from equations (2.63) through (2.65) at the average power condition. An 11-foot core with flattened power distribution was selected for the analysis.

The results of the calculations are given in Table 2-2 in terms of the stability index.*. It is observed that the calculated stability index is sensitive to the non-linear properties of the heat transfer effects, but is relatively insensitive to the non-linear characteristics of the Doppler effect. From these results it can be concluded that in a digital simulation,

*For the investigation an 11-foot core with a flattened power distribution and power density of 84.5 watts/cc was used. Further details can be found in cases 17-23 of Appendix $C$. 
it is important to account for the non-linear properties of the fuel temperature feedback effect as related to the heat transfer mechanism. Since, in general, feedback calculations take considerably longer than the equivalent non-feedback calculations, the relative insensitivity to the non-linear properties of the Doppler effect as such may be of use in the development of temperature feedback methods.

TABLE 2-2

VARIATION OF FEEDBACK

\begin{tabular}{|c|c|c|c|}
\hline Case & $\begin{array}{l}\text { Fuel } \\
\text { Temperature } \\
\text { Feedback }\end{array}$ & $\begin{array}{l}\text { Stability } \\
\text { Index } \\
\left(h r^{-1}\right)\end{array}$ & $\begin{array}{l}\text { Corrected } \\
\text { Stability } \\
\text { Index } \\
\left(h r^{-1}\right)\end{array}$ \\
\hline 1 & Nominal & 0.00764 & 0.0160 \\
\hline 2 & $+20 \%$ Nominal & -0.00372 & 0 \\
\hline 3 & $-20 \%$ Nominal & 0.02087 & 0.0332 \\
\hline 4 & $\partial \mathrm{T}_{\mathrm{F}} / \partial \mathrm{P}=$ Constant & 0.0115 & 0.0205 \\
\hline 5 & $\partial \mathrm{T}_{\mathrm{F}} / \partial \mathrm{P}, \partial \Sigma_{\mathrm{a}} / \partial \mathrm{P}, \partial \Sigma_{\mathrm{R}} / \partial \mathrm{P}=\mathrm{C}$ & 0.0114 & 0.0204 \\
\hline 6 . & $\Sigma_{\mathrm{R}}$ Constant & 0.0356 & 0.02277 \\
\hline 7 & $\begin{array}{l}\Sigma_{a_{1}}=\frac{(1-p)}{p} \Sigma_{R} \\
\text { and } \Sigma_{R} \text { Constant }\end{array}$ & -0.0145 & -0.1232 \\
\hline
\end{tabular}

\subsubsection{Variation of the Removal Cross Section with Fuel Temperature}

In a number of diffusion theory programs, including the TURBO* code, the removal cross section in a two-group formulation is assumed to be independent of the fuel temperature. A correct total neutron balance is maintained by specifying a calculated relationship between the resonance escape probability $p$ and the local power density. The local fast absorption cross section $\Sigma_{a_{1}}$ is then obtained from

$$
\Sigma_{\mathrm{a}_{1}}=\frac{1-\mathrm{p}}{\mathrm{p}} \Sigma_{\mathrm{R}}
$$


where $\Sigma_{R}$ is held constant.

The validity of the above assumption in the digital simulation of spatial xenon oscillations was investigated with the ZEST program. The approximations were simulated in equations (2.64) and (2.65) by adjusting the appropriate coefficient $a_{i}$ and $b_{i}$. An 11-foot core with a flattened power distribution was used for the analysis. The results of the calculations are shown in Table 2-2. It is observed that keeping $\Sigma_{R}$ constant while maintaining the correct variation of $\Sigma_{a_{1}}$ with fuel temperature leads to a significant increase in the growth of the oscillations. This sensitivity to the variation of the removal cross section with fuel temperature is to be expected, since the rate of absorption in Xe-135 is dependent on the absolute thermal flux which, in turn, is dependent on the removal cross section.

The use of an effective variation of $\Sigma_{a_{1}}$ with temperature through equation (2.66) yields results which correspond more closely to the original results although, for this particular problem, the growth rate was underestimated. These results point to the importance of accounting for variations in both fast absorption and removal cross sections with fuel temperature in two-group simulations of xenon-induced oscillations.

\subsubsection{Effect of Temperature Time Lags on Xenon-Induced Instability}

As discussed above in Section 2.2.3, in digital simulation of spatial xenon oscillations it is usual to neglect the finite time lag between power and temperature changes and to assume that both the fuel and moderator temperatures vary instantaneously with power density changes. Chernick has investigated this problem for the fundamental xenon instability. [15] For a negative temperature coefficient, he has shown that the presence of a non-zero time lag increases the fundamental instability. Although the effect was small in the cases considered, within the context of a model analysis, Shotkin has shown ${ }^{[16]}$ that the interaction of the xenon and temperature feedbacks could be neglected in the study of spatial instabilities, except for high fluxes, outside the range of operation of a large PWR. 
Program SPARTA was used to investigate this effect in a large PWR in terms of the space-dependent transfer function formulation. A description of the theory behind this code and an explanation of its use is given in Section 2.5. The magnitude of the feedback time-constant necessary to influence the spatial xenon stability characteristic was sought. Since the fuel temperature timeconstant, $T_{f}$, in a PWR is very short, calculations were performed with $\mathrm{T}_{\mathrm{f}}=0$, and for various values of the moderator temperature time-constant $\mathrm{T}_{\mathrm{m}}$. The 11-foot diameter core had a flattened power distribution. The boron concentration was such as to give a net moderator temperature coefficient of $+0.6 \times 10^{-4} /{ }^{\circ} \mathrm{F}$, giving rise to an unstable core. Values of 1000 and 100 seconds were used for $T_{m}$, 10 seconds being representative of a typical PWR design. The fundamental instability was suppressed in the calculations. The results are shown on Figure 2-12 in terms of the frequency response integrated over half of the core. It is observed that the temperature time lag exerts a stabilizing effect (for a positive moderator temperature coefficient), although this effect is very small. It can be concluded that temperature time lags of the order of a few minutes or less can be neglected in the analysis of spatial xenon oscillations in a large PWR.

\subsubsection{Effect of Delayed Neutrons}

The effect of delayed neutrons on fundamental (space-independent) xenon oscillations has been shown by Chernick [17] to be negligible. A similar situation is expected to hold for space-dependent xenon oscillations.

A SPARTA calculation of the space-dependent transfer function was performed for a core with xenon feedback, with and without delayed neutrons. The core consisted of a 11-foot reflected slab, with a flattened power distribution to simulate diametral oscillations. Fuel temperature and moderator density feedback were included. The boron concentration was $1700 \mathrm{ppm}$, giving a moderator temperature coefficient of $+0.6 \times 10^{-4} /{ }^{\circ} \mathrm{F}$. This case is unstable in the first overtone.

In the frequency range of interest, the space-dependent transfer function was practically independent of the delayed neutrons, showing xenon spatial stability to be insensitive to delayed neutrons in a large PWR. The thermal 


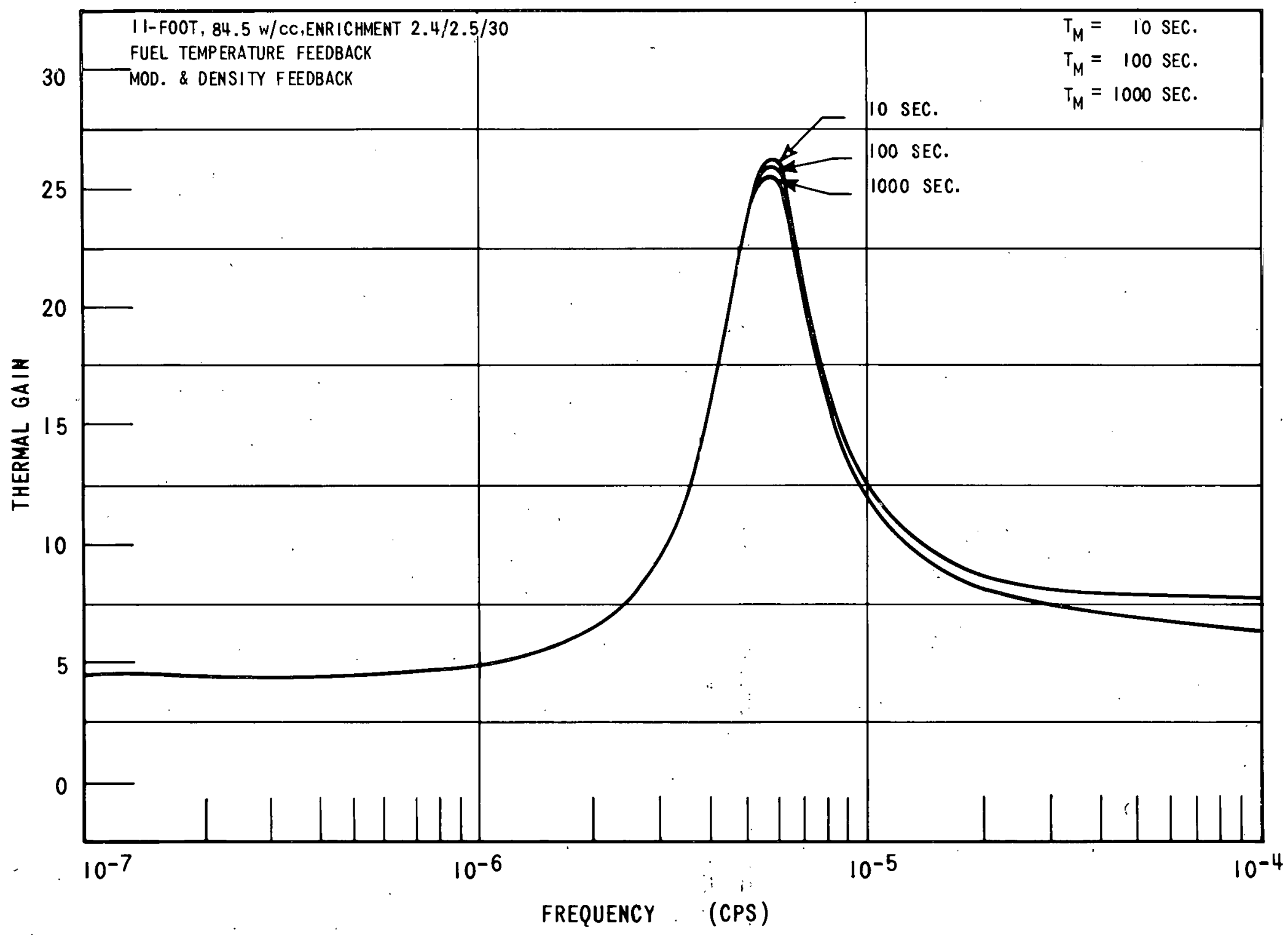

Figure 2-12. Effect of Temperature Time Lags on Xenon-Induced Instability 
gain integrated over half of the core of the resonant. frequency of the first overtone was 2899 without delayed neutrons, and 2897 with delayed neutrons. The corresponding phase shifts were 34.86 and 34.83 , respectively. Although the effect of delayed neutrons is stabilizing, the effect may be entirely neglected.

\subsubsection{Modified Procedures}

Several calculational procedures were briefly investigated. Those described below appear promising but require further analysis. The in-code correction for xenon depletion described in Section 2.3.8.1 is particularly useful because in any investigation of control procedures for xenon oscillations, the time-step can vary and a correction to the stability index after the calculation becomes impossible.

\subsubsection{Modified Depletion Calculations}

The sensitivity of the stability index to the numerical time-step length was discussed in Section 2.3.2. From this it can be gathered that the growth rate of the oscillations is not accurately described when the xenon and iodine equations are solved for a constant flux value during each timestep unless a correction factor is employed to compensate for this fact. The method described in Section 2.3.2 used an analytic type of correction and, as such, is most suitably employed on the results after they have been calculated.

An alternate and more direct approach is to compensate for the variation in flux over the particular time-step by extrapolating the flux and then using this time-dependent flux to deplete the iodine and xenon. This has been done before by Pearce and Roth [10] using a quadratic extrapolation of the form

$$
\phi(x, t)=\phi(x, 0)\left(1+\alpha t+\beta t^{2}\right)
$$

where $\alpha$ and $\beta$ are calculated from the flux of three previous time-steps. Due to the numerical scheme, however, this approach apparently breaks down for a 
combination of too long a time-step with too high a flux. For example, the numerical scheme is unstable when a time-step of two hours is coupled with a flux of more than $10^{14}$ neutrons $/ \mathrm{cm}^{2} / \mathrm{sec}$.

The approach attempted here was to use constant effective flux, $\bar{\phi}(\mathrm{x})$, which was calculated from the integral

$$
\bar{\phi}(x)=\int_{t_{1}}^{t_{2}} \phi(x, t) d t /\left(t_{2}-t_{1}\right)
$$

This was evaluated numerically by using a quadratic fit in the three previous points to integrate the flux through the next time-step, then averaging this over the time-step length. The numerical form of equation (2.68) is then

$$
\bar{\phi}(\mathrm{x})=\left(5 \phi_{\mathrm{N}-2}(\mathrm{x})-16 \phi_{\mathrm{N}-1}(\mathrm{x})+23 \phi_{\mathrm{N}}(\mathrm{x})\right) / 12
$$

where $\phi_{N}(x)$ is the converged flux at the point $x$ for the $N^{\text {th }}$ time-step. This approach has the advantage that because it does not have to perform a Runge-Kutta integration in the xenon calculation, it will be both numerically stable and computationally faster. It does, however, suffer (as does the other method) from the fact that three initial time-steps must be calculated in order to begin the extrapolation. A preliminary analysis tends to show that this method does improve the value of the stability index. However, before this correction could be applied with confidence, a more detailed evaluation would have to be mate.

\subsubsection{Reduction of Number of Iterations Required for Spatial Convergence}

In general, the approximation to the source at the beginning of a new timestep is taken as being the last converged source. As this takes some time to converge, it was decided to try a new source guess. This was derived from a quadratic extrapolation of the converged sources of the last three timesteps. 
The equation used was of the form

$$
S_{N+1}(x)=S_{N}(x)-3 S_{N-1}(x)+3 S_{N-2}(x)
$$

where $\mathrm{S}_{N}(\mathrm{x})$ was the converged source at the point $\mathrm{x}$ for the $\mathrm{N}^{\text {th }}$ time-step. This considerably reduced the number of iterations for some time-steps, although in others no appreciable benefit was obtained. The overall effect, however, was to obtain a considerable reduction in time. On the average, for a 10-foot core with a convergence criterion of $10^{-4}$ in the source, the convergence time was reduced by 40 percent. If the convergence criterion were smaller $\left(10^{-5}\right)$, then this percentage would not be so great, since the number of iterations saved will only be the same as for the $10^{-4}$ criterion.

It was found that the solution using the source extrapolation differed somewhat from that obtained without exercising this option. Part of the reason for the discrepancy will lie in the fact that the solution does not always converge from the same side as that calculated without the extrapolation. As was discussed previously, the solution is quite sensitive to the convergence criterion, hence any modification which affects the parameter will affect the stability of the solution. Although this does not mean that the result is any further from the "true" solution, it does point to the fact that a convergence criterion of $10^{-4}$ is not sufficient. Indeed, to obtain good agreement between two calculations, one with source extrapolation and one without, a convergence criterion of $10^{-5}$ is essential.

\subsubsection{Suppression of Error Due to Finite Convergence}

As mentioned in the last paragraph on extrapolation and discussed earlier in Section 2.3.3; the solution of the diffusion equation coupled to the iodine and xenon equations is quite sensitive to the finite convergence criterion. Also as discussed earlier, the convergence of the source between time-steps in this calculational procedure is always from below when the flux is increasing and from above when it is decreasing. 
One possible way of suppressing this additive effect is by eliminating the consistency of this error. This might be achieved partially in conjunction with the source extrapolation by alternately adding and subtracting sufficient amounts to and from each source guess. In this way, convergence should take place equally from both sides.

\subsection{MODAL ANALYSiS}

Within the framework of linear theory the modal expansion method has received the greatest attention in the solution of the equations of motion, as was described above in section 2.2. The basic elements of the method are now briefly reviewed, and the applicability of modal analysis to PWR stability studies is briefly evaluated and discussed.

In Section 2.3.5 above it was pointed out that the one-group theory offered adequate basis on which to study xenon spatial instabilities in large PWR's. The one-group diffusion will therefore be used as a basis for the modal analysis. As was discussed in Section 2.3.7, delayed neutrons can be neglected in studying spatial xenon oscillations. Finally, as shown by Lellouche, ${ }^{[8]}$ the prompt neutron generation time will be taken as zero. With these assumptions, equations (2.1) and (2.2) reduce to

$$
\nabla \cdot D \nabla \phi-\Sigma_{a}^{*} \phi-N_{x} \sigma_{a}^{\mathrm{x}} \phi+\nu \Sigma_{f} \phi=0
$$

The Xe-135 and I-135 equations in one-group theory are

$$
\begin{gathered}
\frac{\partial N_{x}}{\partial t}=\lambda_{i} \dot{N}_{i}+y_{x} \nu_{f} \phi-\left(\sigma_{a}^{x_{\phi}}+\lambda_{x}\right) N_{x} \\
\frac{\partial N_{i}}{\partial t}=-\lambda_{i} N_{i}+y_{i} \Sigma_{f} \phi
\end{gathered}
$$


The temperature feedback effects are assumed to be prompt and are inciuded in $\Sigma_{a}^{*}$. The validity of this assumption was evaluated in Section 2.3 .6 above. Although the control of spatial instabilities is not the prime concern of this study, it is pointed out that a number of authors have included control rod feedback effects within the content of a modal analysis. $[18,19,20]$

\subsubsection{Geometric and Material Modes}

Ward ${ }^{[1]}$ described a solution of the equations of motion (2.71) through (2.73) in terms of an expansion of the state variables in geometric modes. Randall and St. John ${ }^{[21]}$ extended Ward's analysis to include temperature feedback. These authors expanded the state variables in terms of the eigenvectors of the steady-state diffusion equation -- that is, in terms of the material or clean reactor modes.

For oscillation in the first overtone $\psi_{1}(r)$, the stability index $b$, defined in Appendix $B$, and the period of oscillation $T$, are given according to simple modal analysis, ${ }^{[3]}$ by

$$
\begin{gathered}
b=-\frac{\left(\lambda_{1}+\lambda_{x}+\sigma_{x} \bar{\phi}_{1}\right)}{2}-\frac{a_{x} \sigma_{x} \bar{\phi}_{1}\left(\bar{x}_{1}-Y_{x}\right)}{2\left(\mu_{1}^{2}-a_{T} \bar{\phi}_{1}\right)} \\
T=\frac{2 \pi}{\left[c-\left(\frac{b}{2}\right)^{2}\right]^{1 / 2}} \\
c=\lambda_{i}\left[\lambda_{x}+\sigma_{x} \bar{\phi}_{1}-\frac{a_{x} \sigma_{x} \bar{\phi}_{1}\left(1-\bar{x}_{1}\right)}{\left(\mu_{1}^{2}-a_{T} \bar{\phi}_{1}\right.}\right]
\end{gathered}
$$


where

$$
\begin{aligned}
& \bar{\phi}_{1}=\int \mathrm{d} \underline{r} \phi_{0}(\underline{r}) \psi_{1}^{2}(\underline{r}) \\
& \bar{x}_{1}=\int \mathrm{d} \underline{r} x_{0}(\underline{r}) \psi_{1}^{2}(\underline{r}) \\
& \gamma_{x}=\frac{y_{x}}{y_{i}+y_{x}}=\frac{y_{x}}{y_{T}}
\end{aligned}
$$

In equations (2.77) and (2.78), $\phi_{0}(\underline{r})$ and $x_{0}(r)$ are the steady-state flux and xenon distributions, respectively. The xenon concentration is in units relative to the saturation value. In equation (2.76), $\mu_{1}^{2}$ is the eigenvalue corresponding to the eigenvectur $\psi_{1}$, and $a_{x}$ and $a_{T}$ are coefficients which represent the dependence of $\mathrm{B}^{2}$ on the variation in xenon concentration and flux level, respectively. In the $x-y$ plane, $a_{T}$ represents the effects of fuel and moderator temperature feedback. The remaining notation is standard. Within the framework of linear theory, and for the purpose of uncontrolled calculation, the main disadvantages of the Randall-St. John modal analysis lie in the neglect of modal interaction, and in the difficulty in representing the spatial steady state flux distribution in the analysis. The method is useful for our purposes, however, as it leads to the simple analytical expressions for the spatial stability characteristics in equations (2.74) through (2.76). These equations are used in Section 4 for parametric evaluations of the spatial stability characteristics in large PWR's. In the following paragraphs, the applicability of this sample modal expansion method to large PWR's is briefly evaluated.

\subsubsection{Assumption of Space-Time Separability}

As shown by Pearce, [4] the space shape of the excess xenon distribution changes continuously during a cycle. This results from the local xenon burnup rate, which varies spatially. In the analysis of the stability of the first overtone, however, it is assumed that the excess xenon and flux distributions have the 
same spatial shape. Pearce has shown, [4] however, that the assumptions of space-time separability of the excess xenon distributions as are valid in the limiting case of a small threshold flux could, for example, be associated with a large reactor.

Figures 2-13 through 2-16 show the spatial distribution of the excess thermal flux and xenon concentrations in a one-dimensional reflected slab as calculated with program ZEST (refer to Appendix D). Figures 2-13 and 2-14 are for a 10-foot core, with uniform fuel loading and no temperature feedback. Figures 2-13 and 2-14 are for an 11-foot core with a flattened power distribution and with fuel temperature feedback. Both of these cores are unstable in the first overtone, with stability indices of $0.022 \mathrm{hrs}^{-1}$ and $0.016 \mathrm{hrs}^{-1}$, respectively. The distortion in the excess xenon distribution is apparent in Figures $2-15$ and 2-16. As pointed out by Pearce, [4] the peak of the excess xenon distribution occurs at different times in different regions of the core. The distortion of the excess xenon distribution in a large PWR appears to be small, however.

From Figures 2-13 through 2-16 one can see that in a large PWR, the effect of the reflector is small. The influence of the reflector on xenon instabilities has been studied by Pearce [4] and by Shotkin. [16] Shotkin [16] has shown that the eigenvalues of the perturbed flux in a reflected reactor are lower than those in a bare reactor, leading to the prediction of less stable conditions in a reflected core.

\subsubsection{Moda1 Interaction}

Modal coupling has been discussed by Gyorey, [23,24] Lellouche, [8] and Shotkin. [16] In general, these authors have found that modal interaction is destabilizing in that an analysis which reflects modal coupling will generally overestimate stability. Gyorey and Shotkin $[23,24,16]$ show that as core dimensions increase, modal interaction is enhanced. According to Lellouche, [8] modal interaction is usually small for relatively low flux levels. It should be noted, however, that these authors use for their calculations a Xe-135 absorption cross section, $\sigma_{a}^{\mathrm{X}}$ of $3 \times 10^{-18} \mathrm{~cm}^{2}$. In a PWR, $\sigma_{a}^{\mathrm{X}}$ has an effective value of approximately $1.6 \times 10^{-18} \mathrm{~cm}^{2}$. Since an increase in $\sigma_{a}^{\mathrm{x}}$ leads to a 


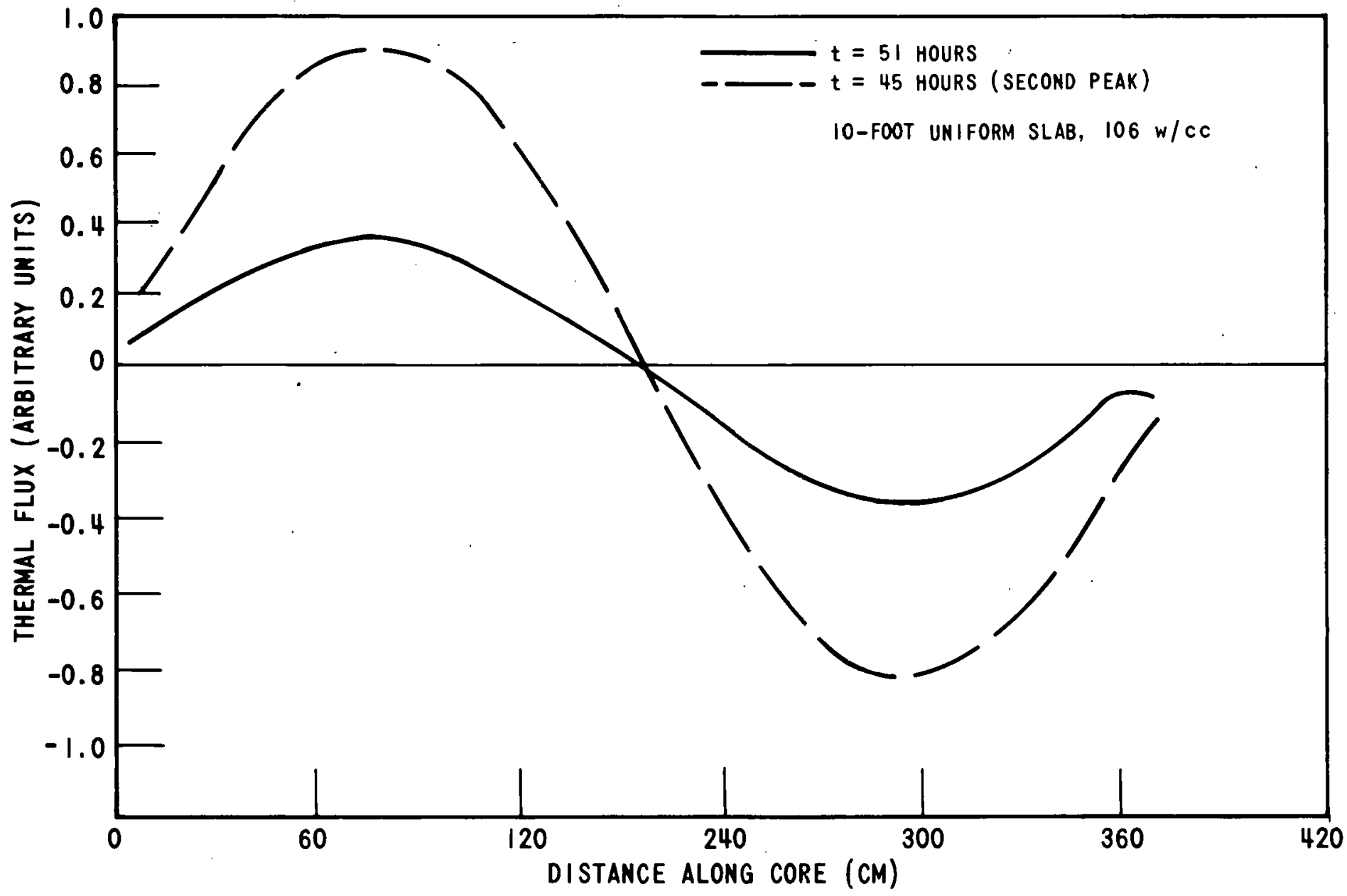

Figure 2-13. Spatial Distribution of Thermal Flux Variations (10-Foot Core) 


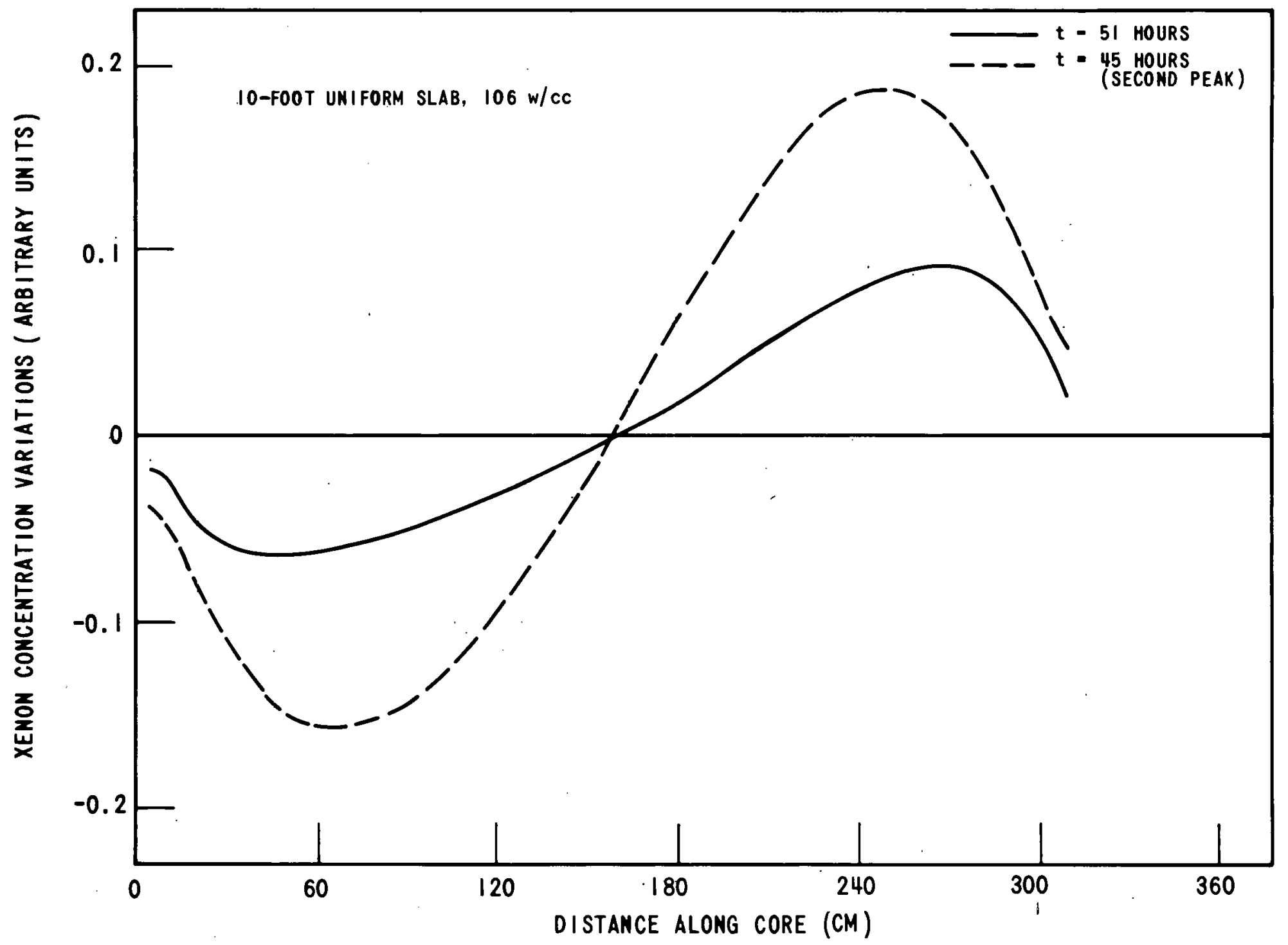

Figure 2-14. Spatial Distribution of Excess Xenon Concentration. (10-Foot Core) 


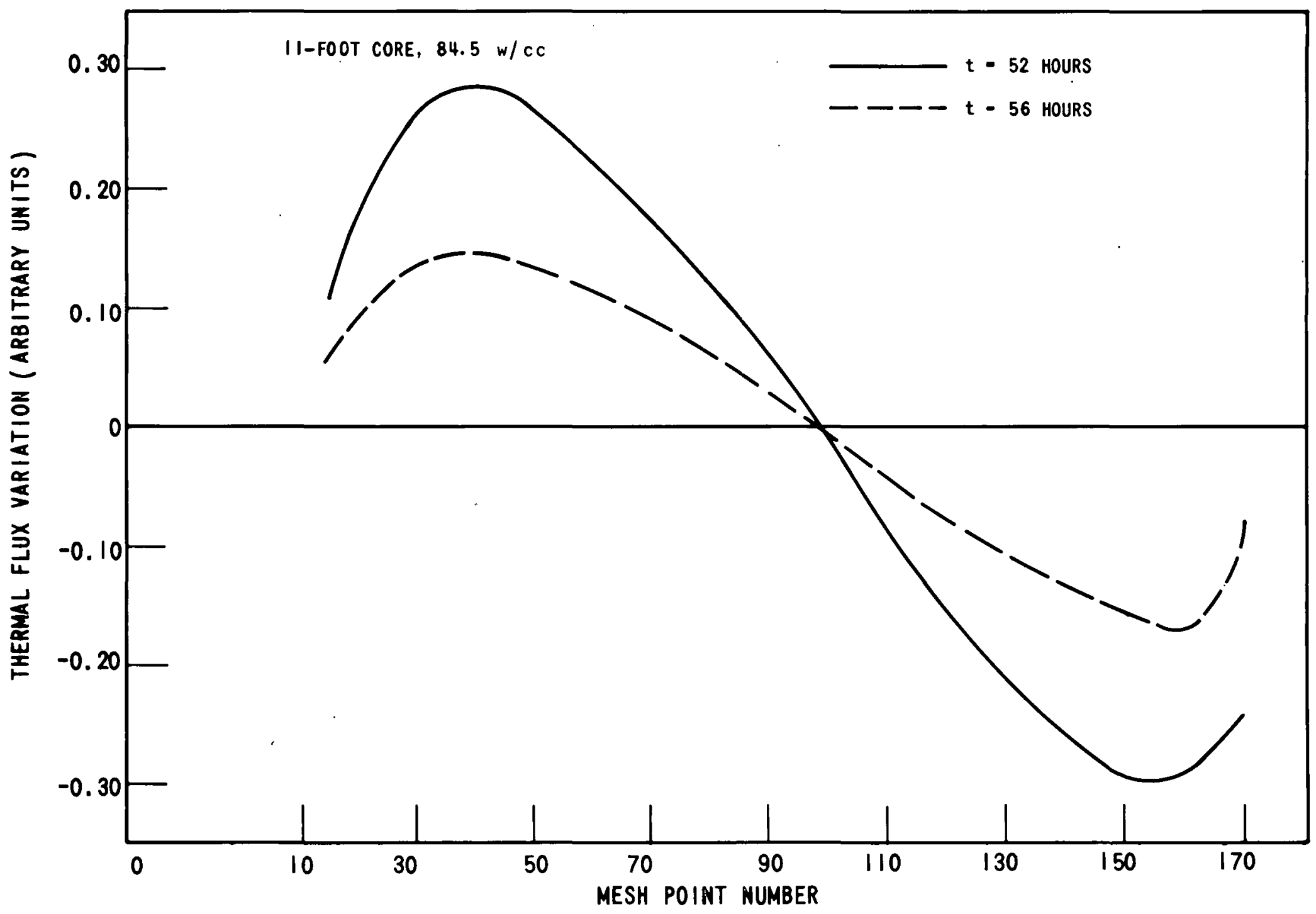

Figure 2-15. Spatial-Distribution of Thermal. Flux (11-Foot Core) 


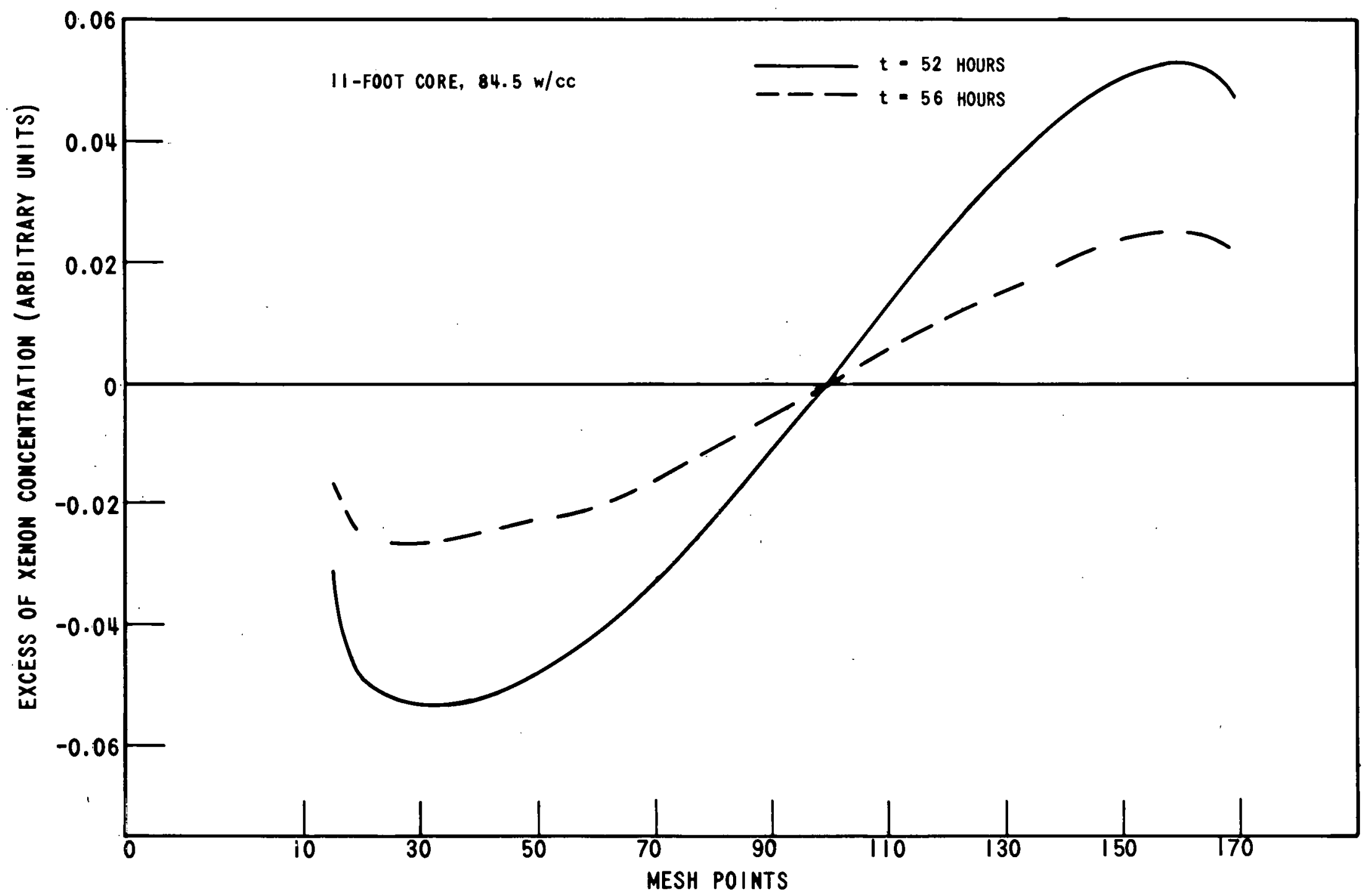

Figure 2-16. Spatial Distribution of Excess Xenon Concentration (11-Foot Core) 
less stable system (refer to Section 4), the cores considered by these authors were much less stable than PWR cores of similar sizes. Therefore it is probable that modal coupling in a large PWR is much less than would be inferred from these calculations.

As pointed out by Gyorey [23] and by Shotkin, [16] flux flattening tends to reduce the amount of modal coupling. For a perfectly flat flux distribution, modal interaction is absent. Due to economic considerations, PWR cores are normally designed to operate with a flattened steady-state power distribution, thus providing some additional justification for neglecting modal interaction.

\subsubsection{Representation of the Steady-State Flux Distribution}

As noted earlier, the spatial stability characteristics are sensitive to the steady-state flux distribution $\phi_{0}(r)$. A major difficulty which arises in using equations (2.74) through $(2.76)$ is the specification of $\bar{\phi}_{1}$ and $\mu_{1}^{2}$. For each new distribution an accurate specification of $\bar{\phi}_{1}$ and $\mu_{1}^{2}$ requires $\phi_{0}(r)$, the calculation of eigenfunction $\phi_{1}(r)$ and the integration in equation (2.78). However, in specifying $\mu_{1}^{2}$, one can define [3] a spatial distribution index $\Lambda$, which gives an indication of the amount of flux flattening in the core.

For a perfectly flat flux, $\Lambda=1$, while for uniform loading in a bare slab, $\Lambda=3$. For the purpose of parametric or sensitivity studies, the use of such a distribution index is adequate. Moreover, as w11l be shown in Section 4.1 the dependence of the stability characteristics on the steady-state flux distribution is very much reduced when the core is operated with a negative net temperature coefficient -- the normal mode of operation. The integral in equation (2.77) can usually be approximated by the average flux $\bar{\phi}_{0}$. It is pointed out here that in analyzing oscillations along a given direction in space, attention should be paid to the flux distribution in the other two directions in specifying a value for $\bar{\phi}_{0}$. The use of a one-dimensional slab to simulate oscillations across an axis assumes that flux is constant in the directions normal to the slab. Pearce ${ }^{[25]}$ has shown that, for simple geometries, the assumption of a flat flux in the directions normal to the slab leads to average flux thresholds higher than would be predicted by a three-dimensional analysis. 


\subsubsection{Spatial Dependence of the Temperature Feedback}

In equations (2.74) and (2.76), the temperature feedback coefficient $a_{T}$, is assumed to be a constant, independent of position in the core. Norinder [20] and Wiberg ${ }^{[18]}$ have raised the question as to the effect of this assumption on the spatial stability characteristics of the core. To evaluate this assumption, the space-dependent transfer function was calculated with SPARTA in a one-dimensional reflected slab with xenon and fuel temperature feedback. The core was 11 feet in length, with zoned enrichments to attain a flattened flux distribution. This core is unstable in the first overtone with a stability index of $0.016 \mathrm{hrs}^{-1}$. In one calculation, the fuel temperature feedback was constant across the core. In a second calculation, the space-dependence of the temperature feedback was explicitly accounted for. In both cases, the fundamental mode of oscillation was suppressed.

Figure 2-17 shows the gain response at the resonant frequency of the first overtone. It is observed that the spatial dependence of the temperature feedback has a small but destabilizing effect on the first overtone.

\subsubsection{Comparison of Modal Theory Results with Diffusion Theory Digital Results}

The modal theory used in Section 4 as a basis for sensitivity and parametric analysis has been compared with diffusion theory calculations. The comparison was made for a 10-foot reflected slab with a uniform fuel loading (2.6 a/० U-235) and no temperature feedback. The diffusion theory calculations were performed with program ZEST, and were corrected for the use of a nonzero time-step length.

The modal calculation and the diffusion theory results are shown in Figure 2-18. For the core studied here, the steady-state distribution is not a true sinusoid because of the flattening effect of the spatial equilibrium xenon distribution and a small reflector effect. Therefore, the distribution index $\Lambda_{1}$ is somewhat less than 3.0, which is the value for a sinusoid. With no temperature feedback the stability index is very sensitive to the flux distribution index $\Lambda_{1}$, as will be shown in Section 4. For the particular core studied here, the value of $\Lambda_{1}$ is estimated to be between 2.8 and 2.9 . 


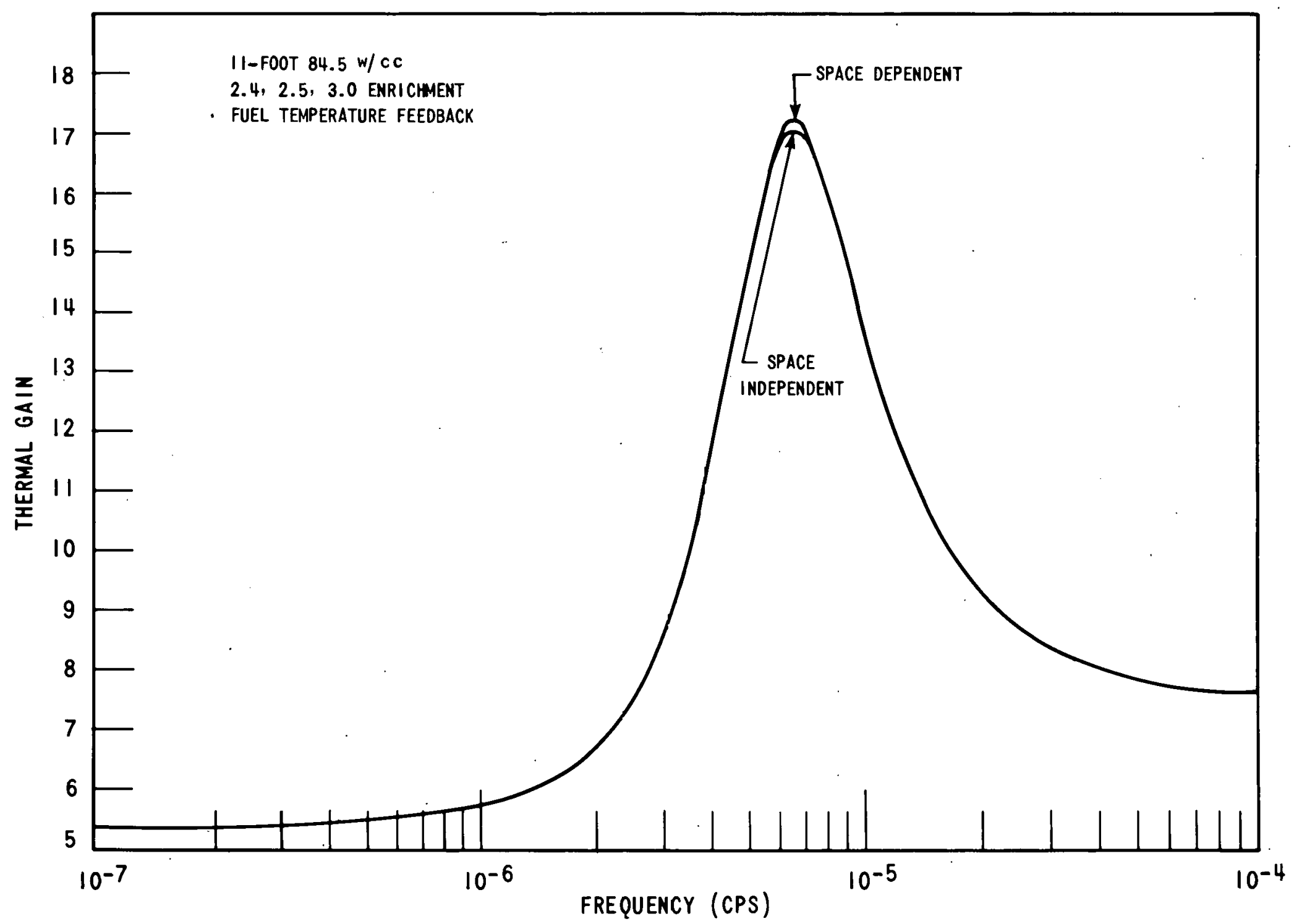

Figure 2-17. Effect of Spatial Dependence of the Temperature Feedback 


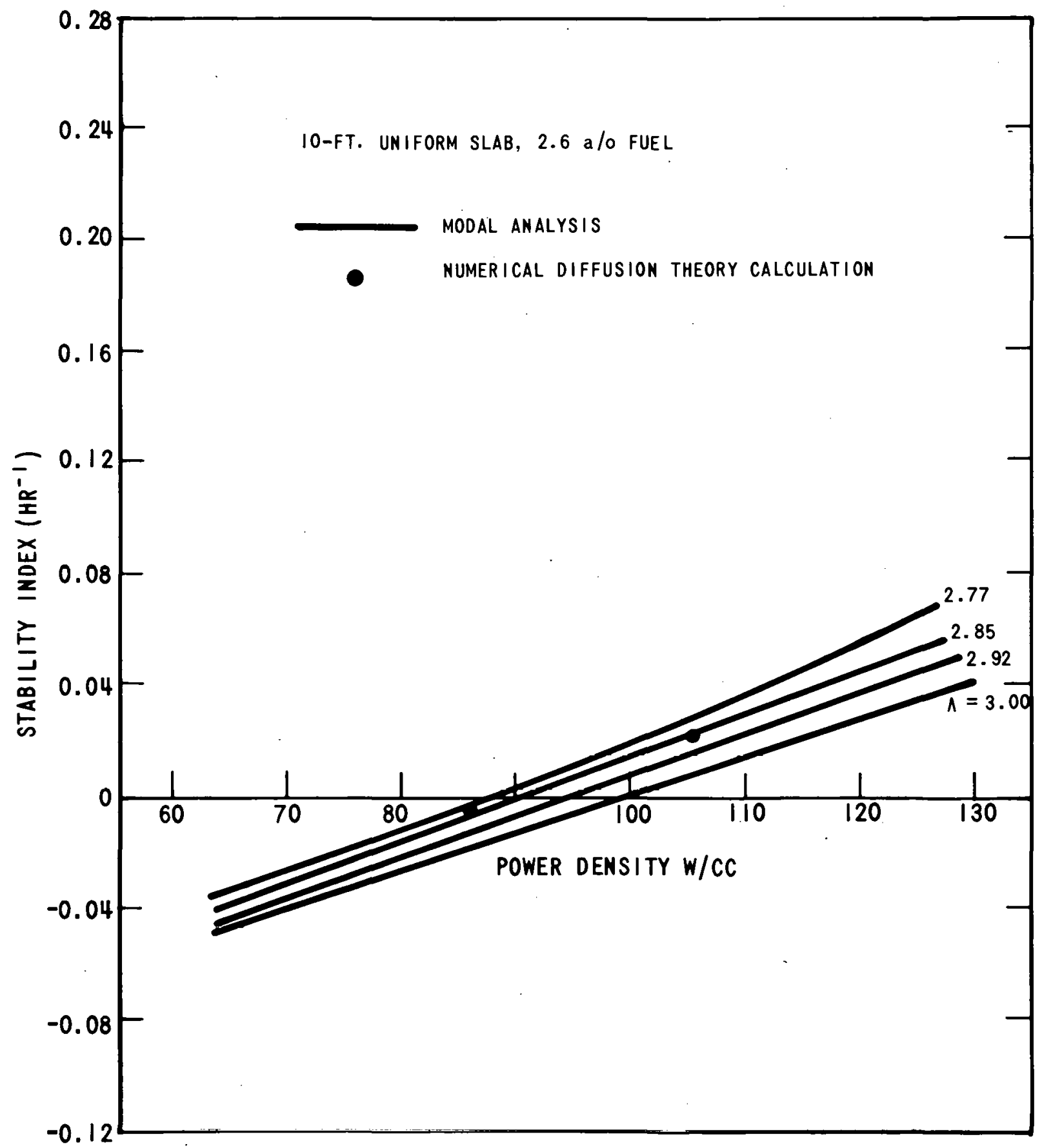

Figure 2-18. Comparison of Numerical Diffusion Theory Calculations and Modal Expansion Analysis 
Some uncertainty should be attached to the diffusion theory results because of the effect of a finite flux convergence criterion. The agreement between the modal analysis and digital calculations exhibited on Figure 2-18 is deemed to be satisfactory. Note, however, that in making such a comparison, it is essential to correct the digital calculations for the effect of a non-zero time-step. This effect was discussed at length in Section 2.3.2. For instance, for a core operating at $106 \mathrm{w} / \mathrm{cc}$, a digital calculation with a four-hour time-step actually predicts a stable condition.

Pearce ${ }^{[4]}$ and Lellouche ${ }^{[8]}$ have found good agreement between modal theory results and analog results. However, the calculations of Barss [9] and Strawbridge ${ }^{[5]}$ revealed some discrepancies between modal results and digital results. Barss ${ }^{[9]}$ noted that digital calculations corrected for a non-zero time-step predicted relatively more stable conditions than did modal calculations. This disagreement may be partly due to the effect of using a finite flux convergence criterion in the digital calculations. As was noted in Section 2.3.3 above, the use of a finite criterion usually leads to more stable conditions. The disagreement noted in reference 5 may be attributed in part to this effect, as well as to the effect of non-zero time-step length.

\subsubsection{Complex Natural Modes}

The expansion in terms of complex natural modes, originally proposed by Kaplan [26] and used extensively by the Bettis Laboratories, ${ }^{[27]}$ possesses the property of finality (no modal interaction) and is generally superior to the above. The basic formulation and the approximation to the complex modes by $\mu$-mode calculation are discussed at length in references 26,27 , and 28 . The analysis, however, is more complex than the simple formulation for geometric modes reported in Section 2.4 .1 and must be implemented numerically. The use of complex natural modes in PWR stability studies is affected by a number of disadvantages. As was noted earlier, in a modal analysis it is awkward to represent realistic multidimensional core configurations and to study realistic control procedures. Within the $\mu$-mode formulation, an additional drawback is the temperature feedback treatment. As is shown in Section 3.5, the fuel temperature feedback provides a very strong 
damping effect on xenon-induced spatial instabilities in large PWR's. The stability characteristics are also very sensitive to the moderator density feedback. The moderator and Doppler feedback effects can be incorporated into a $\mu$-mode calculation by calculating a correction to $\mu$. However, it is felt that digital simulation is probably required to accurately display the complex thermal-hydraulic feedback effects in a large PWR.

\subsection{SPACE-DEPENDENT TRANSFER FUNCTIONS - SPARTA CALCULATIONS}

The theory of space-dependent transfer functions has been described in references 11,29 and 30. Program SPARTA, which calculates the spacedependent transfer function in one-dimensional geometry, is briefly described in reference 11 .

SPARTA solves the linearized form of equations (2.1) through (2.9) with linear feedback. The equations are first Laplace-transformed, and the solution obtained is in the frequency domain through the substitution $s=i \omega$. The solution is given in terms of a groupwise space-dependent transfer function defined as

$$
G_{k}[\underline{r}, M(\underline{r}), s]=A \frac{\psi_{k}(\underline{r}, s)}{f(s)} \quad k=1,2
$$

where $\psi_{k}(r, s)$ is the Laplace-transform of the groupwise flux perturbations; $f(s)$ is the transform of the time-dependence of the perturbation; the function $M(r)$ represents the spatial distribution of the perturbation. In equation (2.80), A is a normalization constant. The delayed neutrons, the time delays in the feedback mechanism and the prompt neutron generation time are all accounted for in the model, the only approximation being that of linear theory.

The application of the space-dependent transfer function concept to spatial stability -- and in particular to xenon instabilities -- has been briefly discussed in reference, 11 . In an unstable core, the frequency response calculated by SPAR'IA can be associated with a particular solution of the equations of motion for a forcing function of the form $e^{i \omega t}$. Oscillatory 
spatial instability is characterized in a Bode or frequency response plot by a series of peaks or resonances in the gain response of the transfer function. Each peak corresponds to a given spatial mode of oscillation (fundamental, first overtone, etc.). At each resonant frequency, the real and imaginary parts of the transfer function exhibit the spatial distribution of the particular mode. Thus, the gain will peak for all spatial points in the core with a different magnitude, except at the node points for that mode, where no peaks appear. In studying spatial instabilities, the fundamental can be explicitly suppressed by specifying a spatial distribution of the perturbation which does not include a fundamental component.* The magnitude of the peak in the gain response is directly related to the degree of instability of a particular mode. An increase in the peak corresponds to a greater instability and vice-versa. In this report, the SPARTA code was used to obtain relative results; that 1s, the variations in the magnitude of the peak with changes in parameters have been used as indications of the sensitivity of the stability characteristics of those parameters.

Although SPARTA solves the diffusion equations directly without formal modal expansions, the space-dependent transfer function displays the time behavior of the modes. These modes are natural modes in that their spatial distribution corresponds to the modes naturally excited in a digital simulation and, presumably, in a real. reactor. These modes, as postulated by Wiberg, correspond also to Kaplan's complex modes. [26]

The space-dependent transfer function approach, as formulated in SPARTA, offers a number of advantages relative to the modal approach outlined above in Section 2.4. Major among these is the spatial treatment, which does not depend on the assumption of a modal expansion of all state variables, but is similar in nature to the spatial treatment in a digital simulation. It also depends on the feedback treatment, which is much more complete and explicit.

\footnotetext{
*In a symmetrical core, this is best achieved by specifying uniform perturbation in both halves of the core which are of the same magnitude, but of opposite signs. The only modes which can be excited are then the first, third, etc. ... overtones.
} 
The main advantages of SPARTA over digital simulation lie in the savings in computer time. A typical calculation, with temperature feedback, which requires about 20 minutes by digital simulation. would require only about one minute with SPARTA. Since SPARTA does not depend upon a discrete time mesh in formulation, the errors noted in digital simulation which arise from finite time-step lengths and flux convergence criteria do not come into play. Finally, short time delays such as temperature delays and delayed neutron production are accounted for in SPARTA, while they are neglected in digital simulation.

In addition to the drawbacks of linear theory, a major disadvantage of the SPARTA approach lies in the difficulty of incorporating feedback control -particularly when dead-band control is involved. SPARTA is therefore best suited to studying the inherent stability characteristics of a core. In view of the explicit feedback treatment and treatment of delayed neutrons, both of which are used by SPARTA, the space-dependent approach has been used in this work primarily to evaluate the sensitivity of the spatial stability characteristics to the treatment of temperature feedbacks and of delayed neutrons (refer to Sections 2.3 .6 and 2.3.7). 


\section{SECTION 3}

SPACE-TIME CHARACTERISTICS OF XENON-INDUCED OSCILLATIONS IN LARGE PWR'S

This section describes the space-time behavior of xenon-induced oscillations in the plane normal to the direction of coolant flow in large PWR's. This plane will be noted as the $x-y$ plane. The analysis is restricted to freerunning oscillations; that is, the problem of control of spatial instabilities is not directly considered here. A knowledge of the spatial behavior of free-running oscillations is, however, a prerequisite to any attempt to design an adequate detection and control system.

Although PWR cores approximate a right circular cylinder, they exhibit a noncircular, staggered boundary in the $x-y$ plane, with non-uniform zoning inside the boundary. The stability characteristics and the nature of spatial oscillations in such a system are best studied through actual simulation, using multidimensional diffusion codes.

Maximum use has been made of two-dimensional, $x-y$ diffusion-theory calculations in the analysis. The spatial progräm TURBO* [7] used in the analysis was described in Section 2.3.1.1. Although TURBO* can account for the spatial fuel temperature feedback, the computer time is excessive when such option is selected. Thus, it has been necessary to limit most of the two-dimensional calculations to core conditions which neglect temperature feedbacks. Also as a result of the relatively large amounts of time required by two-dimensional codes, it has been necessary to take time-steps of four hours in all the TURBO* calculations. However, the results of these have been corrected to zero time-step. A number of one-dimensional calculations, with and without temperature feedback, were performed to supplement (when necessary) the twodimensional calculations. As was noted in Section 2.3.1.2, diametral oscillations in the $x-y$ plane can be relatively well simulated in onedimensional slab geometry. 
The basic geometry and design data used in the calculation are summarized in Appendix A. Except when explicitly stated, a spatial oscillation was excited in all cases by inserting a poison perturbation locally in the core. The perturbation simulated a control rod motion, and was homogenized over the area of one fuel assembly. Except where noted, the perturbation was introduced in the core for one hour. Subsequent oscillations were observed at regular time intervals following removal of the perturbation. In all cases, the perturbations were introduced in a core operating with equilibrium xenon. The emphasis in the following paragraphs is on the spatial distribution of the power after the core has been perturbed, on the effect of the magnitude and the size of the perturbation and on the effect of temperature feedback.

\subsection{SPATIAL CHARACTERISTICS OF OSCILLATION IN THE X-Y PLANE}

The results of a large number of two-dimensional $\mathrm{x}-\mathrm{y}$ calculations have shown that the mode of oscillation which is most easily excited in a PWR core are the oscillations across a diameter. In section 3.2 it is shown that the shifting of a localized perturbation anywhere around the core results merely in a rotation of the nodal diameter. Precessing oscillations along the azimuthal angle can be excited, but require a series of perturbations at different positions and times. The following paragraphs describe the influence of core size, steady-state power distribution and temperature feedback on the spatial behavior of diametral oscillations.* A summary of stability indices and oscillations periods is given in Appendix $C$.

\subsubsection{Diametral Oscillations}

The following figures display the space-time behavior of xenon-induced oscillations in a typical large PWR design. The core has an equivalent diameter of 11 feet. Three-zone enrichments were selected to yield an. approximately flat steady-state distribution. Detailed design characteristics for the core can be found in Appendix A. Temperature feedback was not

*The sensitivity of the stability characteristics, such as stability index and oscillation period to changes in core parameters, is discussed in Section 4. 
included in these calculations because the core is significantly unstable. As will be shown in Section 3.5, the core under its normal operating condition is close to the stability threshold when fuel temperature feedback is accounted for.

A perturbation was introduced along an axis of the equilibrium core $\left(0^{\circ}-180^{\circ}\right)$, approximately $2 / 3$ from the core center. The reactivity worth equivalent for this perturbation was $1 \times 10^{-4}$. After removal of the perturbation, timesteps of four hours each were used to analyze the ensuing oscillations.

Figure 3-1 shows the resulting oscillations or assembly-average-power to core-average-power for two core locations ( 0 and 180 degrees) $2 / 3$ out from the core center. The non-linear saturation effects are apparent when the oscillations become large. (Non-linear effects are further discussed in Section 3.6.) The oscillations are essentially diametral, with a nodal diameter along the $90^{\circ}-270^{\circ}$ axis.

Figures 3-2 through 3-4 show the $x-y$ power distribution as contour maps for 28 hours, 36 hours, and 52 hours, respectively, after removal of the perturbation. The distribution shown on Figure 3-3 is essentially the steady-state distribution. Such plots are very useful in determining the spatial characteristics of the oscillations in time in the two-dimensional plane. As such, they are ultimately useful in specifying the detection and control requirements of the core.

Note that the figures represent the distribution of the local power density. Since different enrichments are used in the three zones, the spatial flux distribution is somewhat different than that in Figures 3-2 to 3-4. The spatial distribution of the power density and the location of the hot-spots in time are, however, the major considerations for the designer.

\subsubsection{Effect of Initial Power Distribution}

The sensitivity of the stability characteristics to the steady-state power distribution of the core is evaluated and discussed in Section 4. The present section displays the spatial power distribution in the $x-y$ plane following a perturbation for a number of different initial power distributions. 


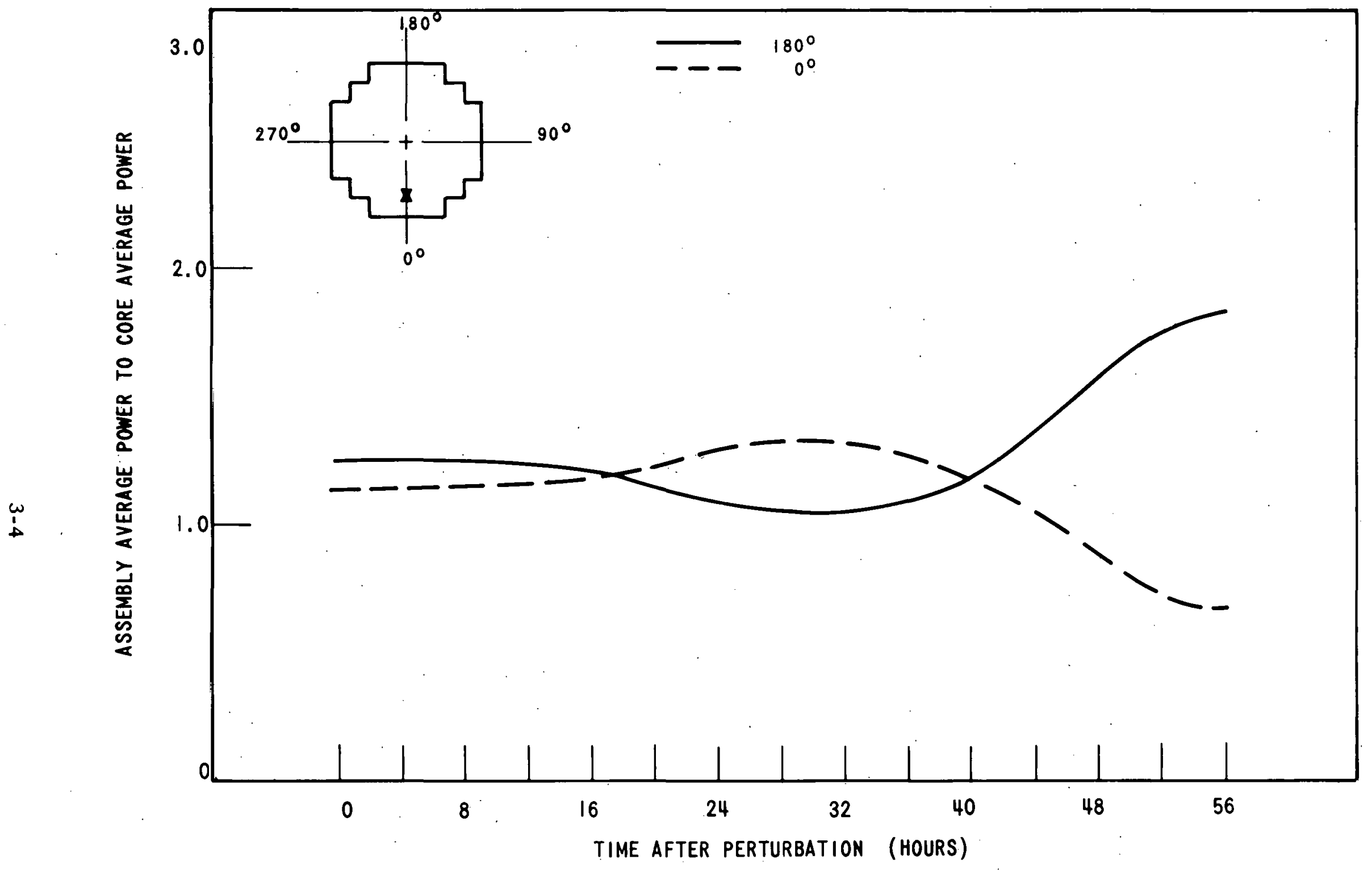

Figure 3-1. Xenon-Induced Oscillations for Poison of Reactivity Worth of $1 \times 10^{-4}$ 


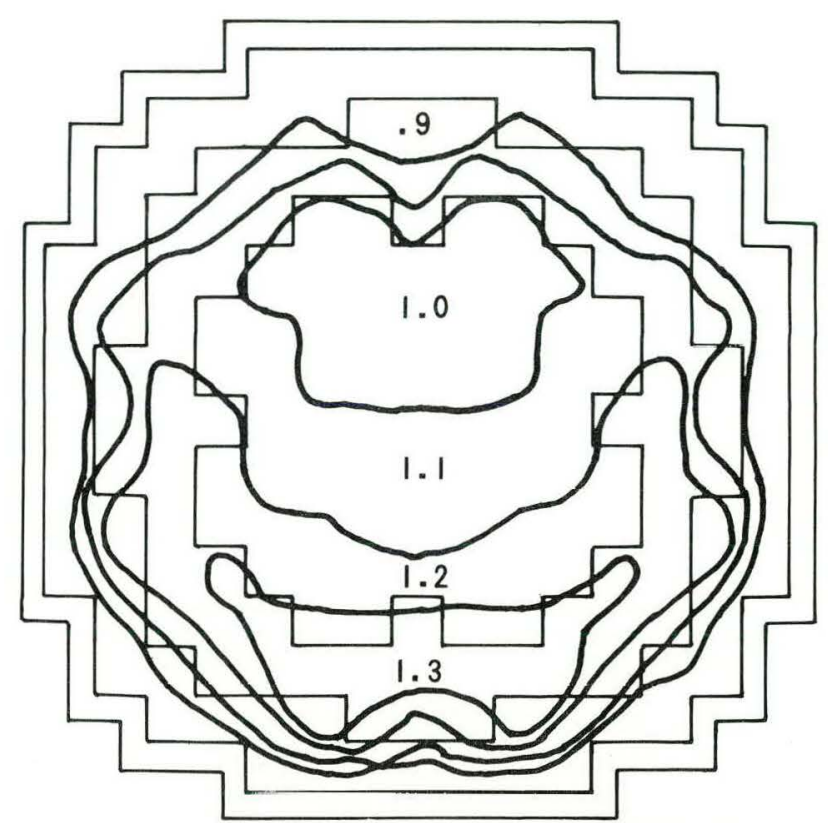

Figure 3-2. Contour Power Distribution for 1/10 Perturbation, 28 Hours after Removal of Perturbation

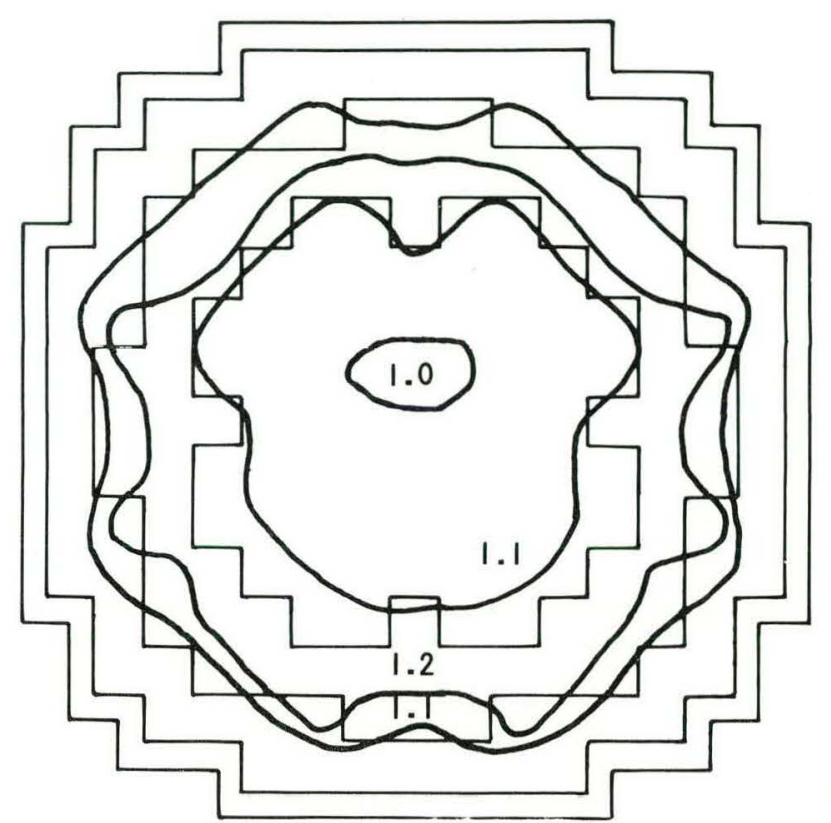

Figure 3-3. Contour Power Distribution for 1/10 Perturbation, 36 Hours after Removal of Perturbation

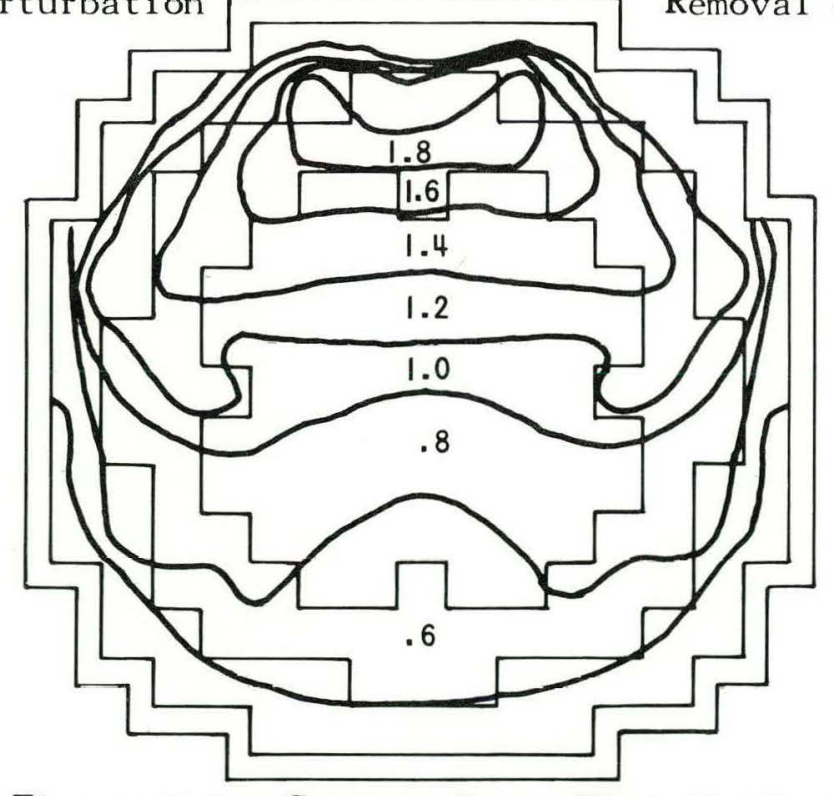

Figure 3-.4. Contour Power Distribution for 1/10 Perturbation, 52 Hours after Removal of Perturbation 
For a given size core, fuel management requirements lead to varying power distributions from the initial core loading to achievement of the equilibrium cycle. Design optimization normally will require flattened power distribution. A core configuration of interest here is one with a checkerboard enrichment pattern. Such a core configuration is shown in Figure 3-5. The steadystate power distribution for this checkerboard core does not differ greatly from that for the three-zone core discussed above except for small discontinuities in power at the assembly interfaces. As will be noted in Section 4.2, the stability characteristics of these two cores are quite similar. Figure 3-6 compares the steady-state power distribution of the checkerboard core with that of the three-zone core. The equivalent curves for the checkerboard core spatial distributions 8 and 32 hours after removal of the perturbation are shown in Figures $3-7$ and $3-8$.

The $x-y$ power distributions for 16 and 28 hours after removal of the perturbation are shown in Figures 3-9 and 3-10 for the checkerboard core. The reactivity equivalent of the perturbation in this case was $9 \times 10^{-4}$, resulting in relatively larger oscillations. Temperature feedback was not accounted for in these calculations. Note that the differences between these curves and those of Figures 3-2 and 3-3 are due to the sharply fluctuating nature of the power distribution.

In recycled cores, power distributions which exhibit triple peaks along a diameter are possible, particularly at the beginning of a given cycle. Two cores with different triple-peak distribution were examined. For these cores, four-zone enrichments were selected to yield the desired power distributions. The steady-state distributions along a diameter of the core are displayed in Figures 3-11 and 3-12. In these calculations, the reactivity equivalent of the perturbation was $9 \times 10^{-4}$ and temperature feedback was neglected. These cores are therefore quite unstable. Contour plots of the power distribution in the two cores at 8,16 , and 28 hours following removal of the perturbation are shown in Figures 3-13 through 3-18. Note that the oscillations are still essentially diametral in nature, although the spatial distributions are markedly different from those described above in Section 3.1.1 for the three-zone core. Additional results for a number of varying initial power distributions can be found in references 31 and 32 . 
$X$ ENRICh-2.1\%

ENR I CH-2.6\%

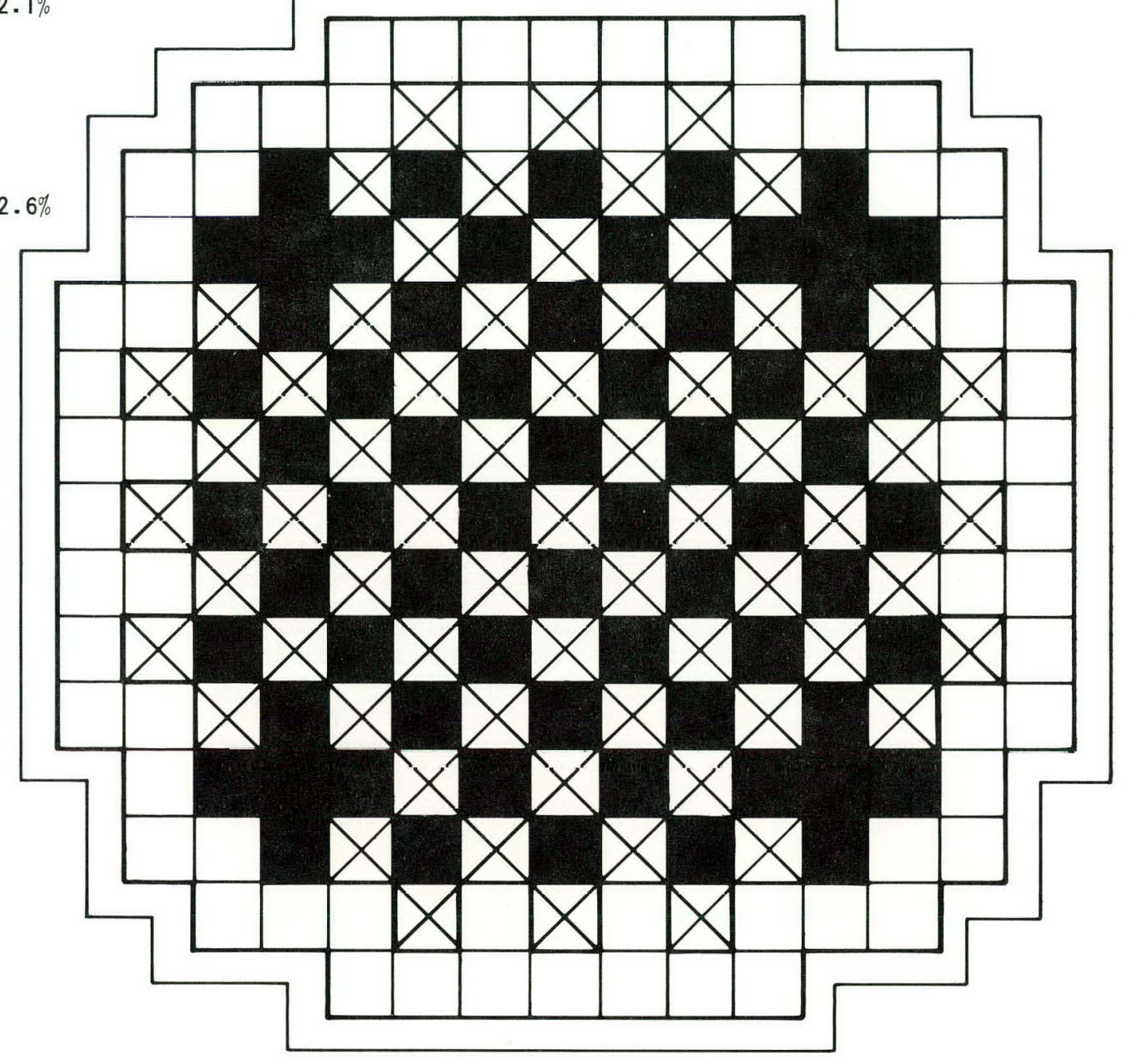

Figure 3-5. Enrichment Pattern for Checkerboard Core 


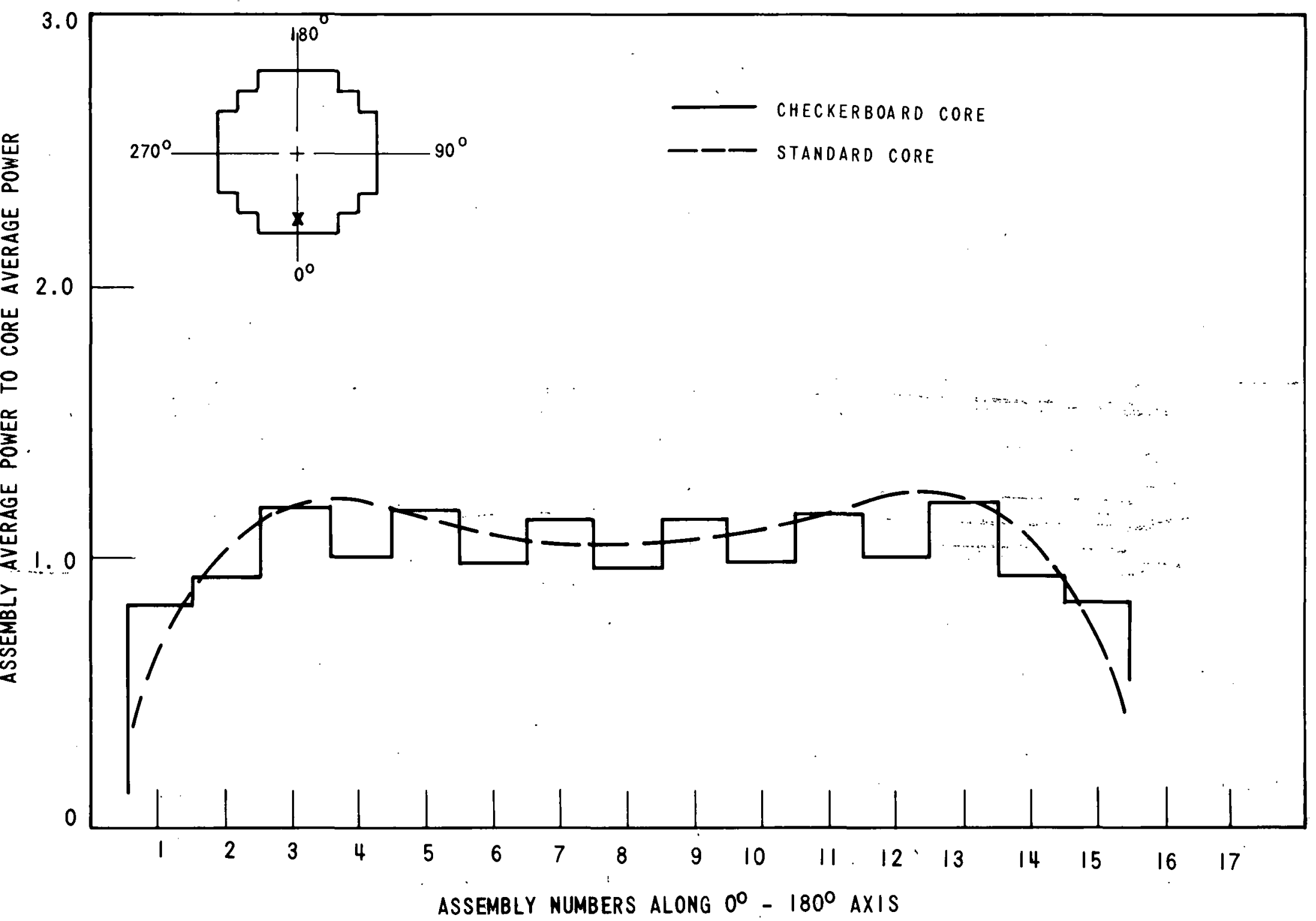

Figure 3-6. Initial Power Distribution for Checkerboard Core 


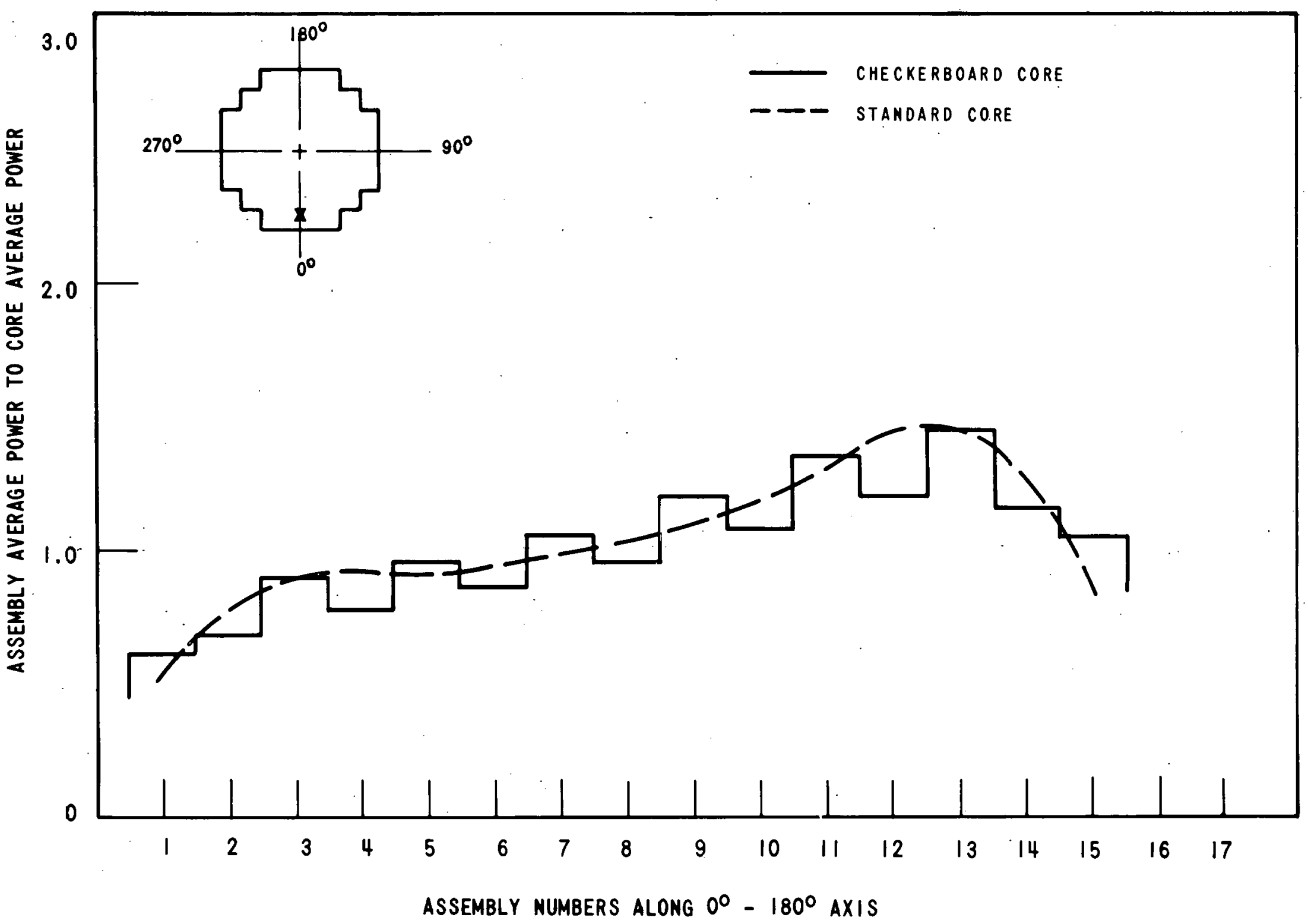

Figure 3-7. Power Distribution for Checkerboard Core, 8 Hours after Removal of Perturbation 


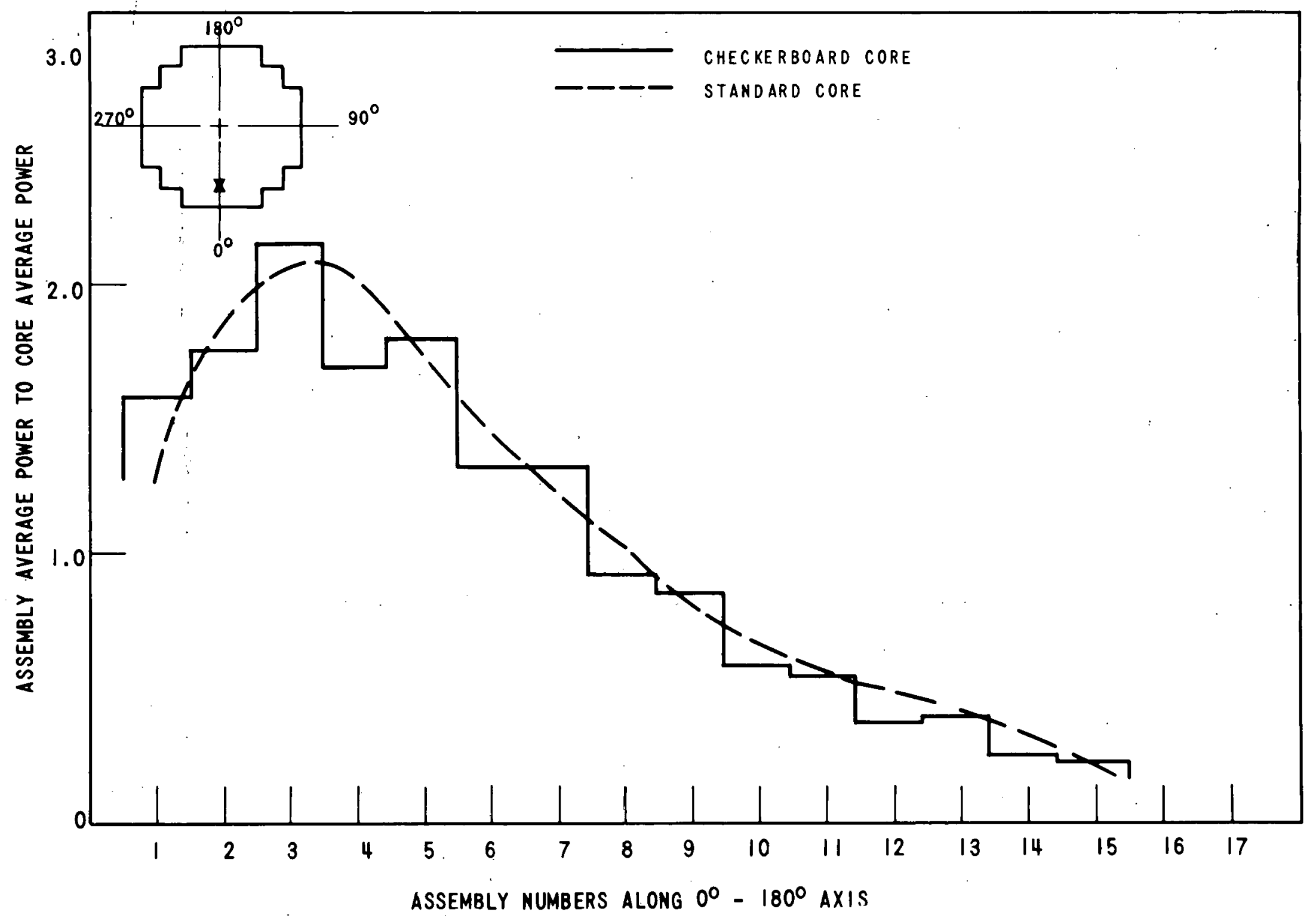

Figure 3-8. Power Distribution for Checkerboard Core,

32 Hours after Removal of Perturbation 


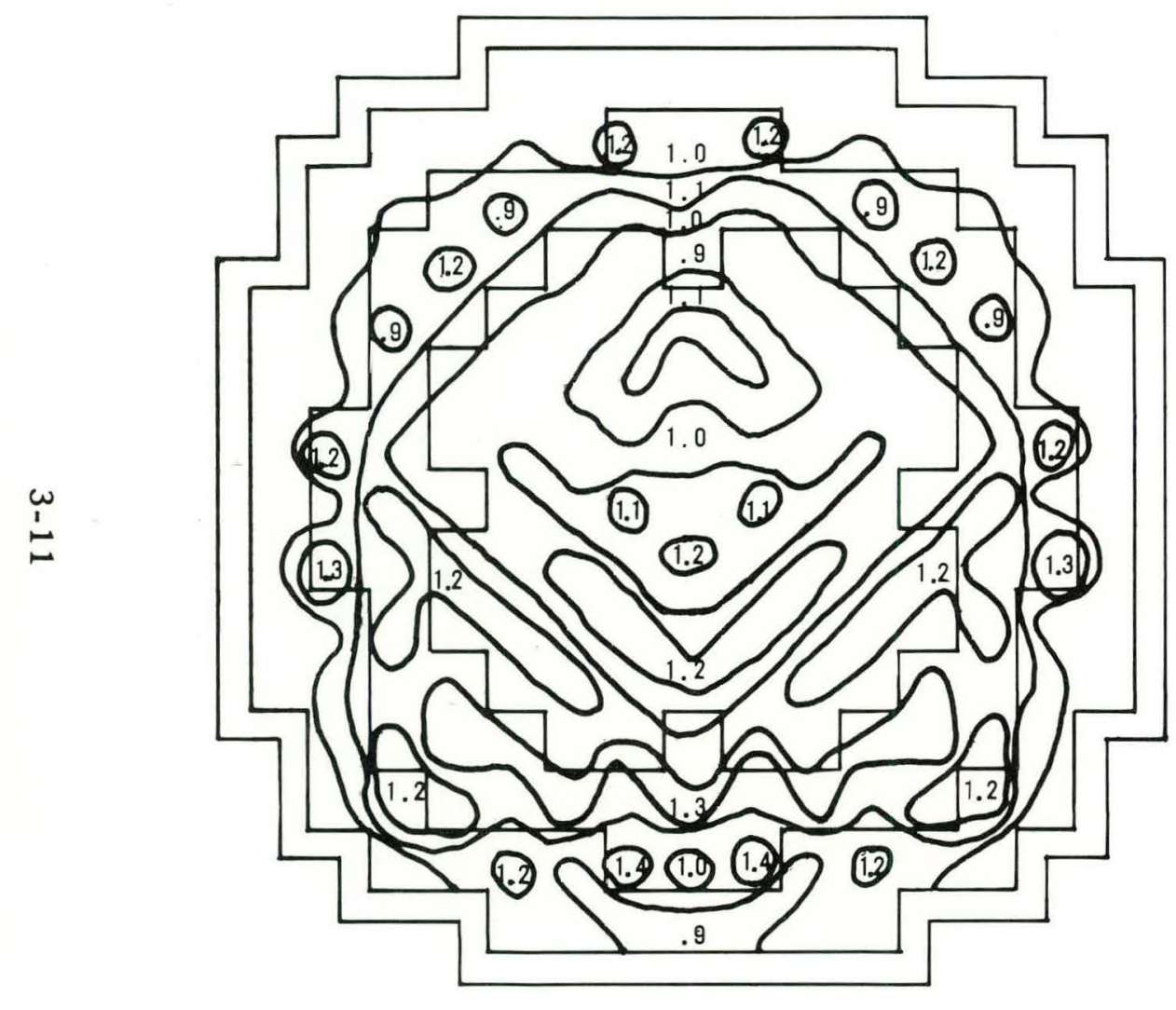

Figure 3-9. Contour Power P1 ot for Checkerboard Core, 16 Hours after Removal of Perturbation

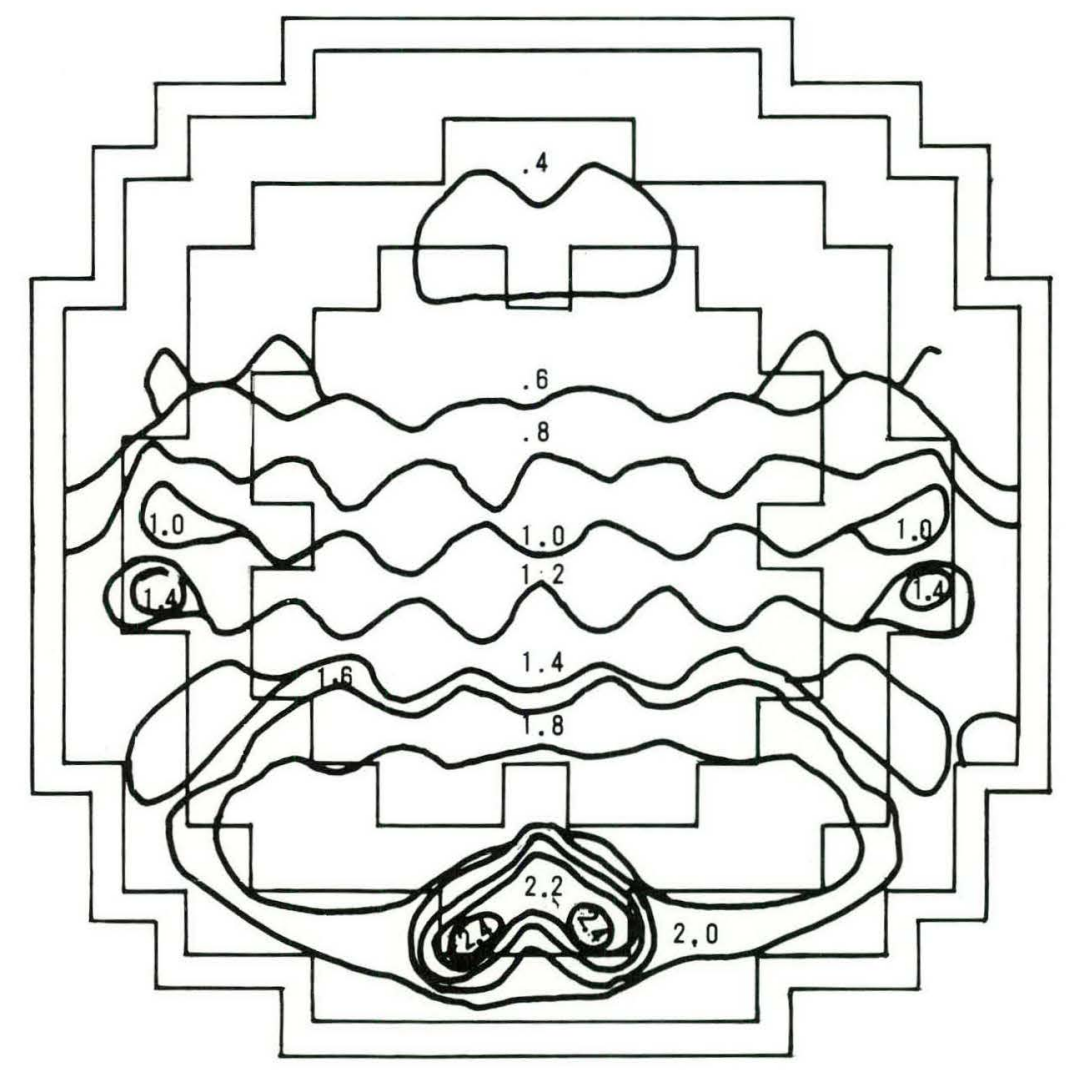

Figure 3-10. Contour Power Plot for Checkerboard Core, 28 Hours after Removal of Perturbation 


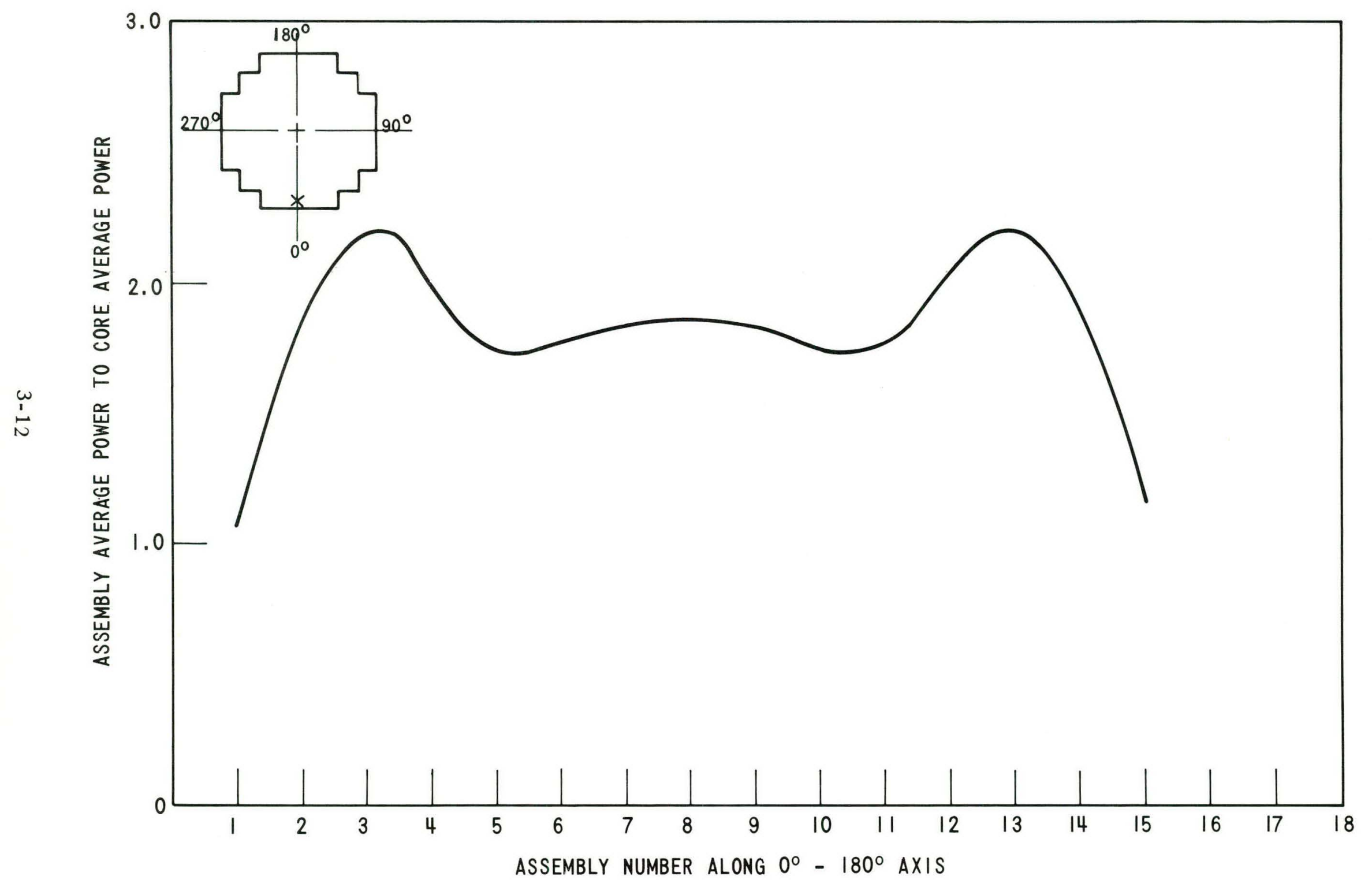

Figure 3-11. Initial Power Distribution for Triple Peak Core Number 1 


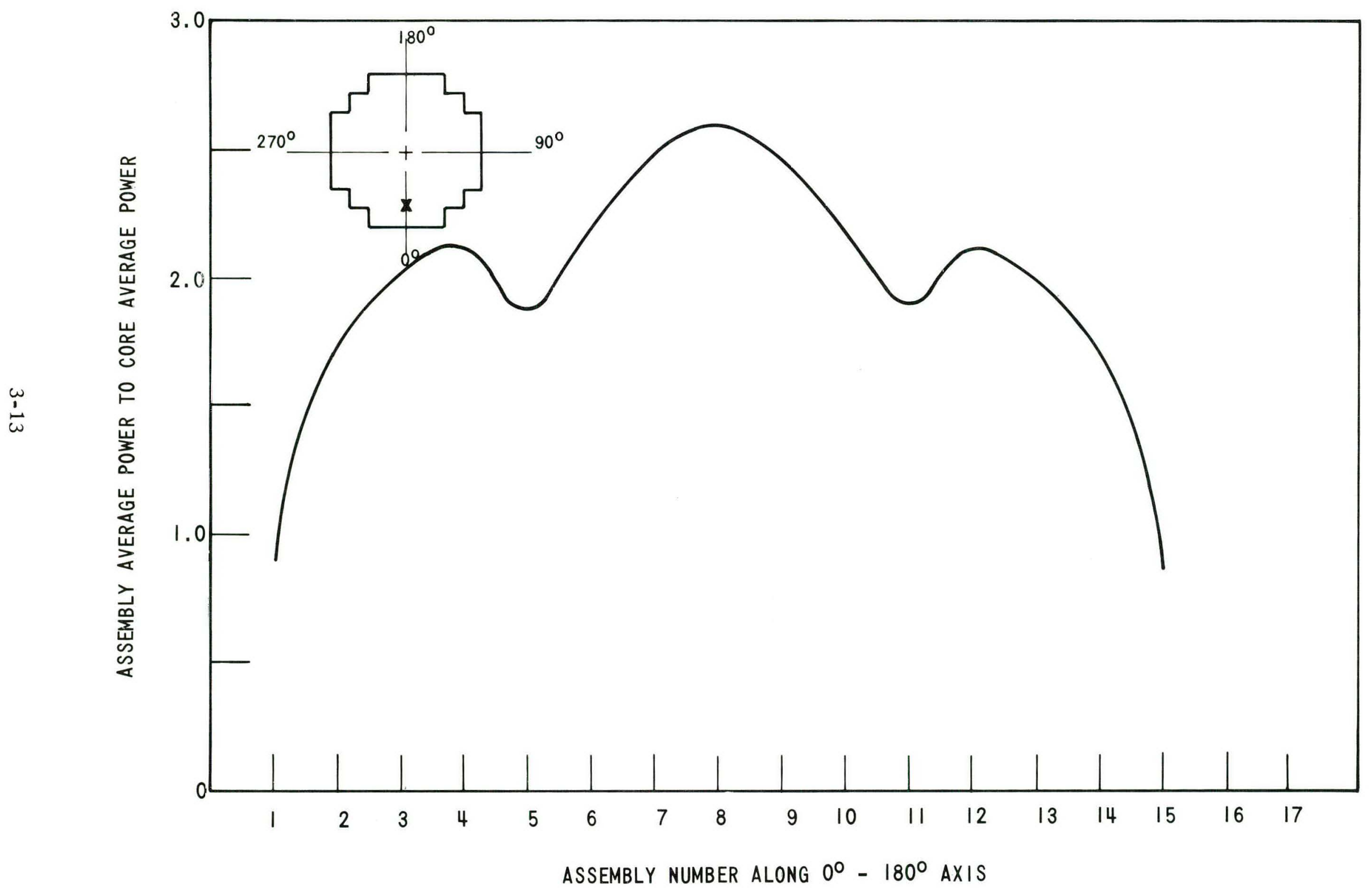

Figure 3-12. Initial Power Distribution for Triple Peak Core Number 2 


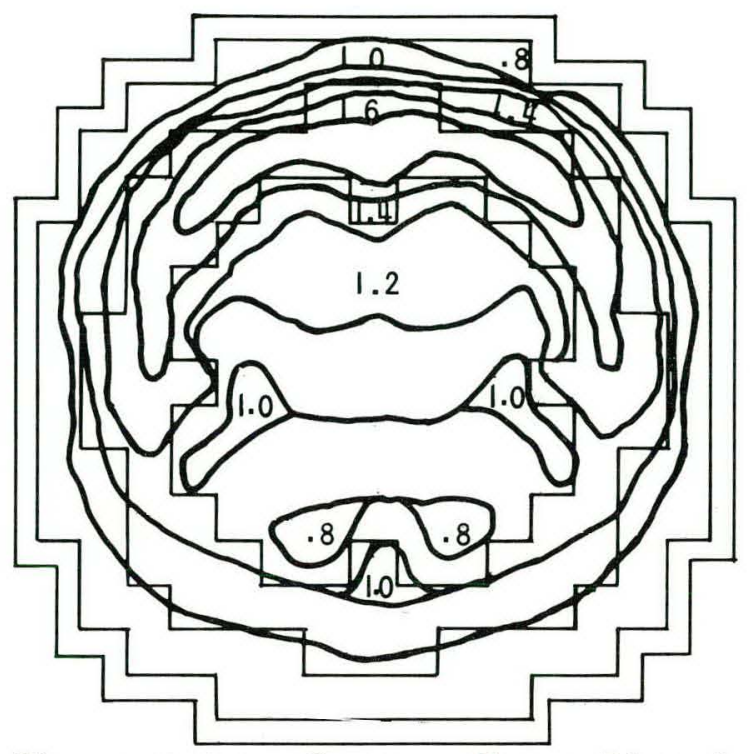

Figure 3-13. Contour Power Plot for Triple Peak Core Number 1, 8 Hours after Removal of Perturbation (All Power Values are Relative)

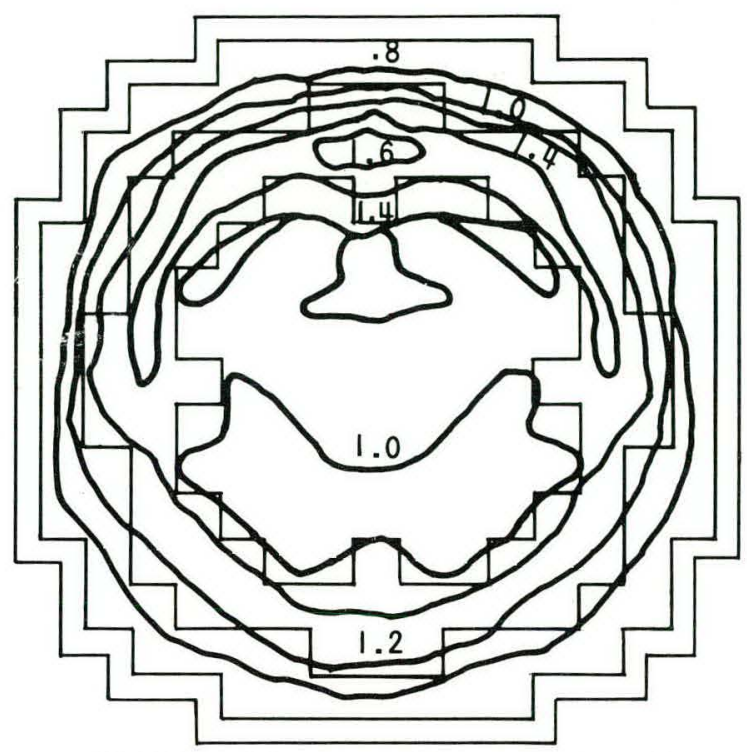

Figure 3-14. Contour Power Plot for Triple Peak Core Number 1, 16 Hours after Removal of Perturbation (A11 Power Values are Relative)

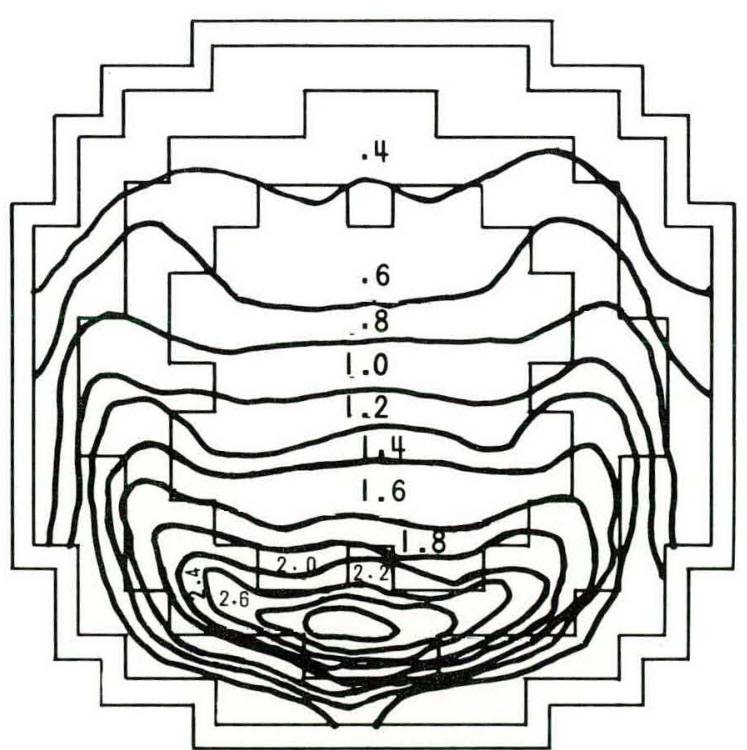

Figure 3-15. Contour Power Plot for Triple Peak Core Number 1, 28 Hours after Removal of Perturbation (Al1

Power Values are Relative) 

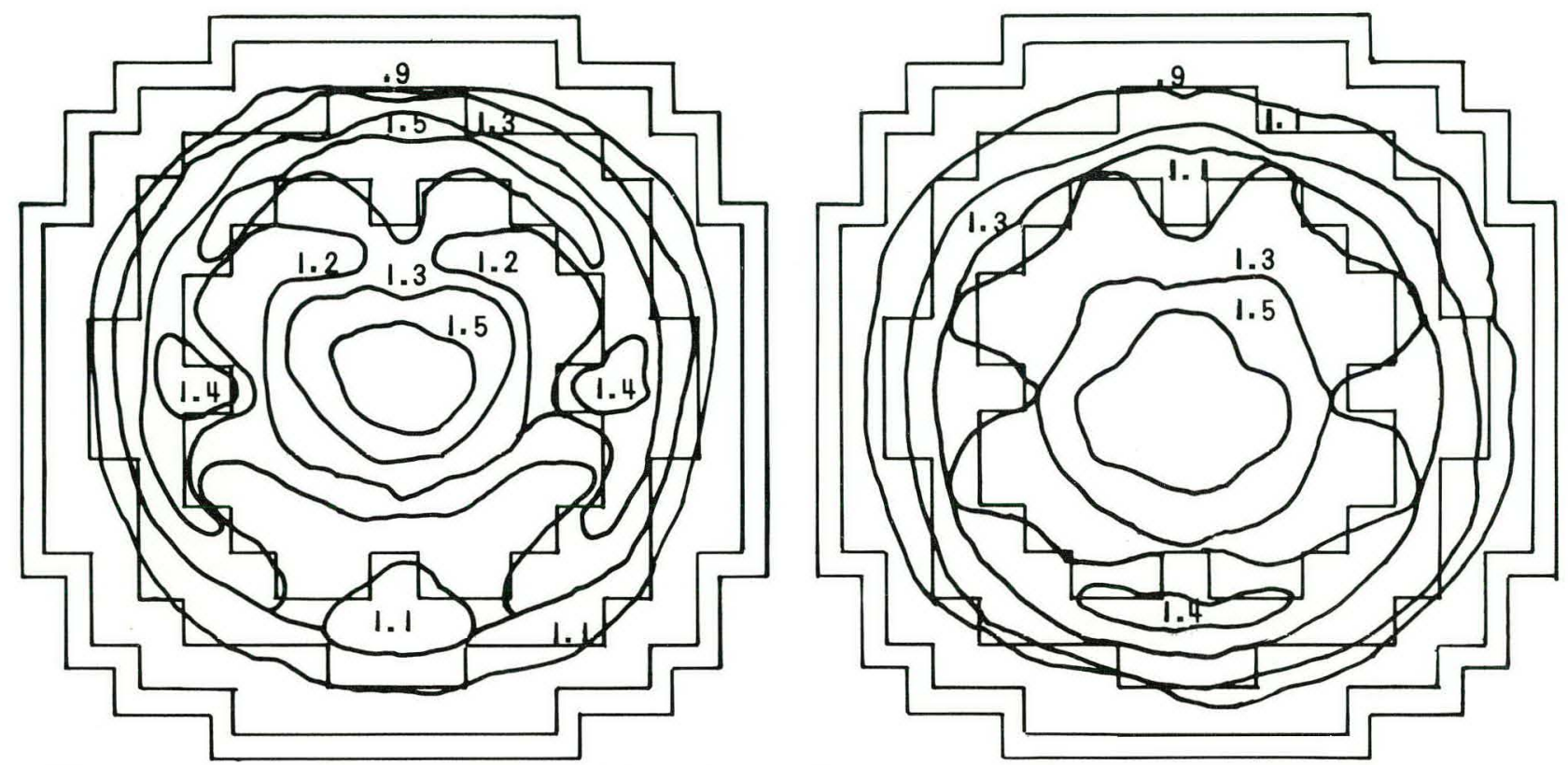

Figure 3-16. Contour Power Plot for Triple Peak Core No. 2, 8 Hours after Removal of Perturbation

Figure 3-17. Contour Power Plot for Triple Peak Core No. 2, 16 Hours after

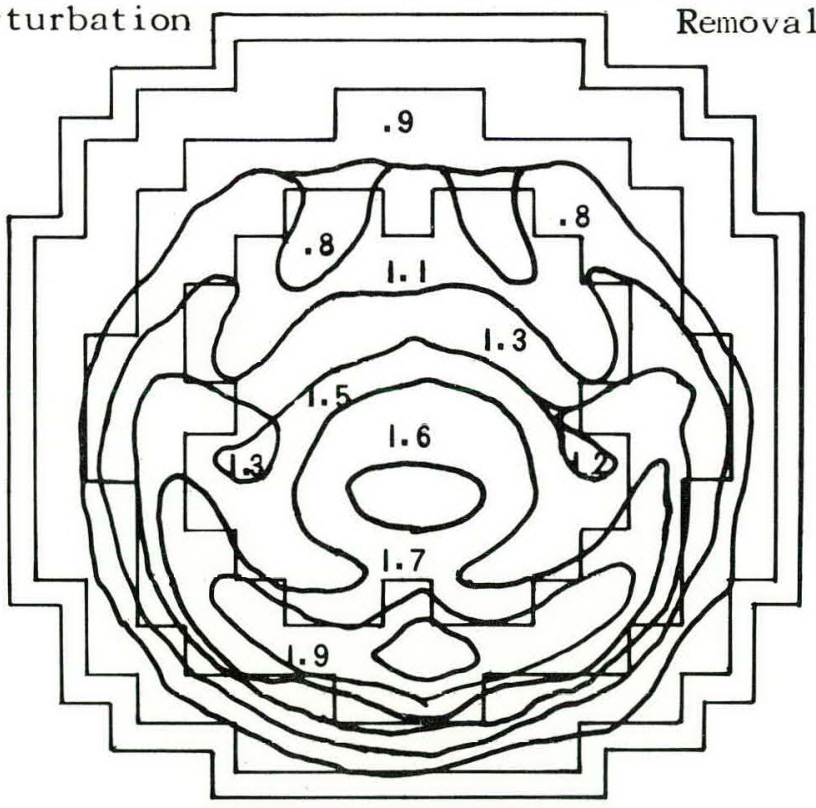

Figure 3-18. Contour Power Plot for Triple Peak Core No. 2, 28 Hours after Removal of Perturbation 


\subsubsection{Effect of Core Size}

The spatial oscillation characteristics described above in Sections 3.1 .1 and 3.1.2 were observed in an 11-foot diameter core. The spatial behavior of the oscillations are also observed for a 9-foot diameter core. Three-zone enrichments were again selected to yield an approximately flat power distribution. Without temperature feedback, this core was approximately on the stability threshold with a time-step length of 4 hours. Although this core is stable at normal operating conditions with temperature feedback, it is still of interest to evaluate the behavior of the spatial oscillations in a smaller core.

Figures 3-19 through 3-21 show contour plots of the power distribution at 20, 32, and 44 hours following removal of the perturbation. The steady-state distribution for this core is approximately that shown in Figure 3-20. Two-dimensional calculations were also performed for 13-foot and 15-foot cores. Machine-time considerations, however, precluded the inclusion of fuel temperature feedback. The resulting oscillations were therefore very large, and were dominated by non-linear effects. Further discussion of the characteristics of these oscillations is given below in Section 3.6. Spatial stability characteristics for a 10-foot core are given in reference 32.

\subsubsection{Effect of Leaving the Perturbation in the Core}

In general, the perturbation was inserted for one hour and then removed, so that the oscillations were propagated in a symmetrical core. In this case, however, the spatial characteristics of the oscillations in the three-zone, 11-foot core with a flattened power distribution were studied under a condition where the perturbation was left in the core permanently after its insertion. Temperature feedback was neglected and the reactivity equivalent of the perturbation was $9 \times 10^{-4}$, thus resulting in relatively large divergent oscillations. Figure 3-23 shows the power oscillations along the $0^{\circ}-180^{\circ}$ axis, while Figures 3-24 through 3-26 show contour plots of the $x-y$ power distribution 13, 21, and 29 hours after insertion of the perturbation. It is observed that the power fluctuations on the side of the perturbations are considerably depressed, while the oscillations on the opposite side were not greatly affected. 


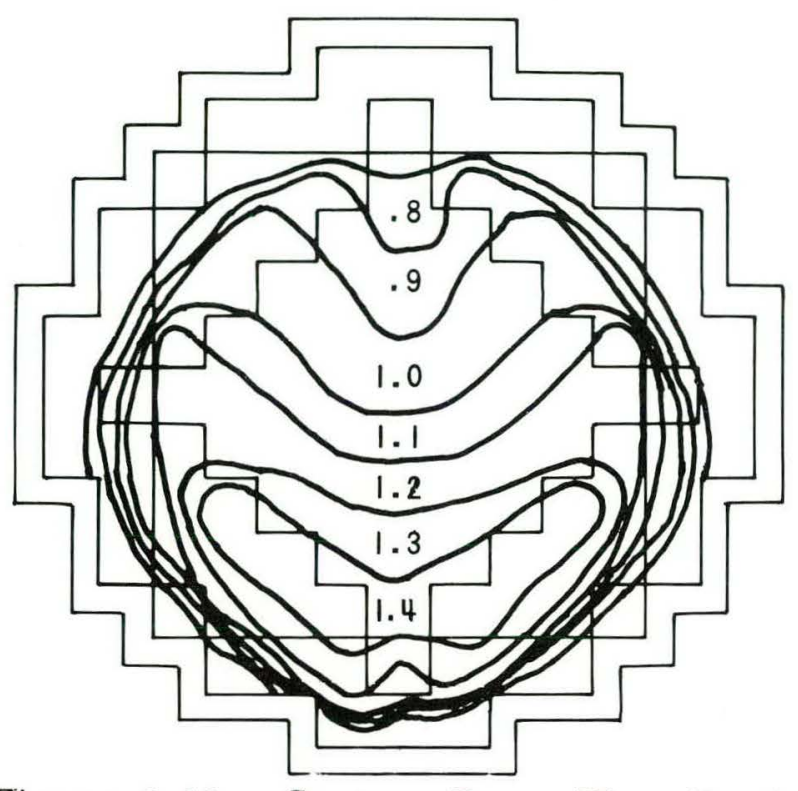

Figure 3-19. Contour Power Distribution for 9-Ft. Core, 20 Hours after Removal of Perturbation

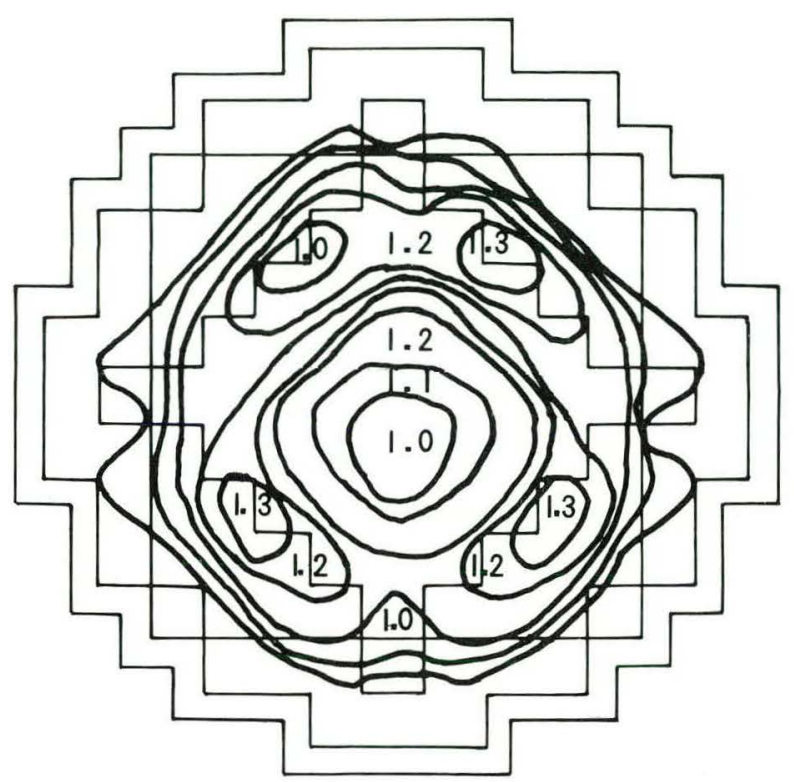

Figurc 3-20. Contour Power Distribution for 9-Ft. Core, 32 Hours after Removal of Perturbation

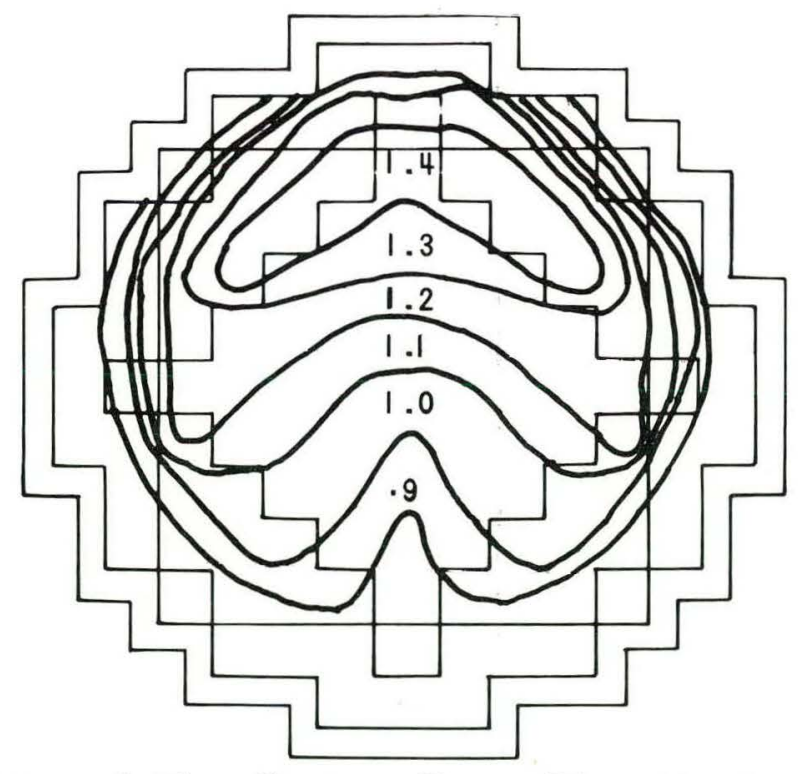

Figure 3-21. Contour Power Distribution for 9-Ft. Core, 44 Hours after Removal of Perturbation 


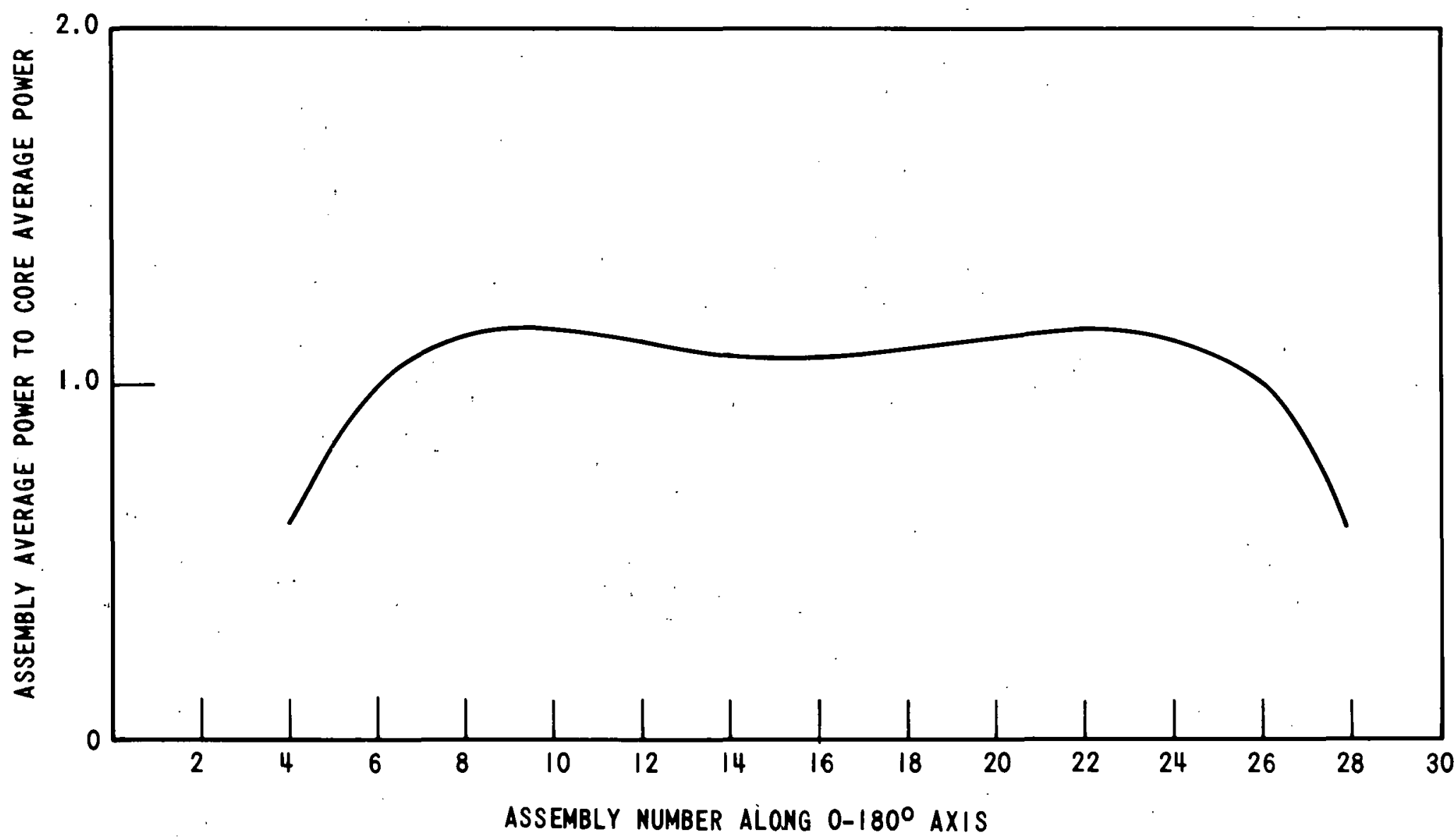

Figure 3-22. Steady-State Power Distribution along $0^{\circ}-180^{\circ}$ Axis for 9-Foot Core 


$$
= \pm
$$




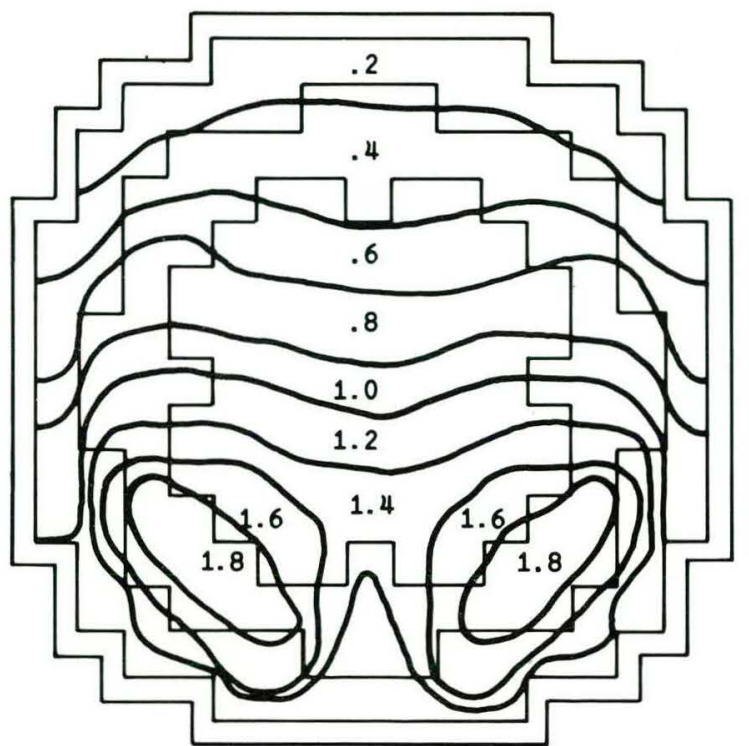

Figure 3-24. Contour Power Distribution for Perturbation Left in 13 Hours After Insertion of Perturbation

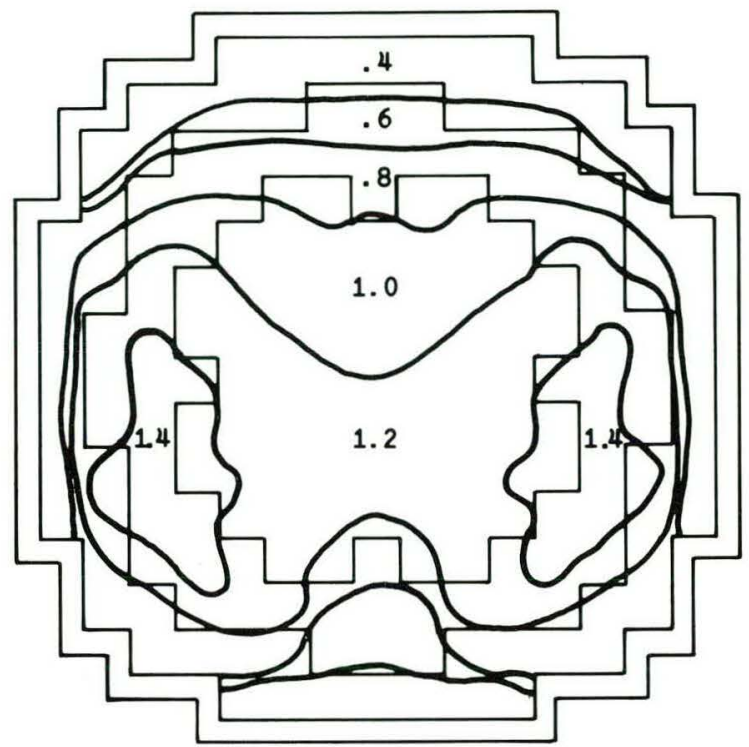

Figure 3-25. Contour Power Distribution for Perturbation Left in 21 Hours After Insertion of Perturbation

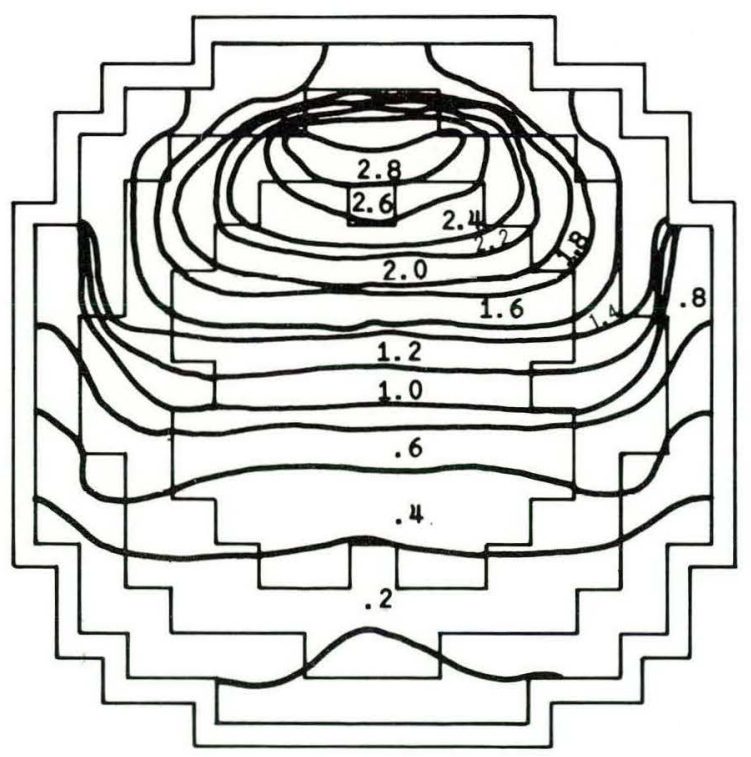

Figure 3-26. Contour Power Distribution for Perturbation Left in 29 Hours After Insertion of Perturbation 
For comparison, the results of the calculation where the perturbation was removed after one hour are shown on Figure 4-18. It is seen from Figure 3-23 that the bulk of the power is produced from the side away from the perturbation.

The contour plots in Figures 3-24 through 3-26 indicate that power peaking tends to propagate from side to side along a circular path on both sides of the $0^{\circ}$ - $180^{\circ}$ axis, rather than along this axis -- as was the case for the oscillations in a symmetrical core.

\subsection{INFLUENCE OF THE PERTURBATION LOCATION}

Knowledge of the steady-state (unperturbed) conditions of a core are important for the determination of the inherent spatial stability characteristics of the core. For a given core, however, the characteristics of the oscillation which follows a perturbation are dependent on the magnitude and location of the perturbation. In terms of a classical modal expansion of the flux shape, only those modes can be excited which are present in a modal expansion of the perturbation. A series of calculations with TURBO* were performed in a given core to evaluate the flux response in space-time to perturbations at different locations.

In the first of these calculations, the perturbation was placed on a 45-degree diagonal relative to the principal axes in order to determine the sensitivity of oscillations of an asymetrically-placed perturbation. A similar calculation was performed with the perturbation placed on a diagonal inclined at 22-1/2 degrees to one of the principal axes. From the results of the above and the case in which the perturbation was placed on a principal axis, it would appear that the stability characteristics of the core are not strongly influenced by the azimuthal location of the perturbation; consequently, other investigations can be made using half-core symmetry with a subsequent reduction in computer storage and running time. Unless otherwise stated, this was what was used for all other calculations.

For the calculation, an 11-foot core with an average enrichment of 2.5 percent and a mesh width of $10.8 \mathrm{~cm}$ was used. The equilibrium power distribution was considerably dished, making the resulting oscillations quite divergent. The 
graphs of the oscillations, however, as shown in Figures 3-27 and 3-28, can be compared with the oscillations described in Figure 4-18 in which the perturbation is placed on a major axis.

The effect of simultaneously placing two perturbations in the reactor -- one on each major axis -- was investigated. These rods were left in an 11-foot core with flattened power distribution for a period of one hour and then removed. The subsequent spatial power distributions were calculated every four hours and some of these are shown in Figure 3-29 through 3-32. Note that the power oscillates along the diagonal bisecting the perturbations. Although, in effect, there is twice as much poison in the core as in the usual calculations, the power oscillations appear to peak only slightly higher.

In addition to investigating azimuthal variations, radial variations in poison position were looked at. The core was again 11 feet in diameter but had a flatter power distribution due to an average fuel enrichment of 2.6 percent. Figure 3-33 shows the power oscillations following a perturbation at a position $2 / 3$ from core center along a radius (Case $i$ ), and at the edge of the core (Case iv). The power oscillations in this and the following figure are those occurring at the symmetric points $2 / 3$ from core center along the axis $0^{\circ}-180^{\circ}$. Note that with the perturbation located at the edge of the core, the magnitude of the oscillations is still relatively large. However, the core was found to be quite insensitive to a perturbation placed in the reflector. Figure 3-34 shows the power oscillations following a perturbation at a position 1/3 from core center (Case iii), and at the core center (Case ii). The core center is located at a nodal point of the first geometric azimuthal mode, and $i t$ is seen that this particular mode is not excited in Case ii. Note also that with the perturbation located at the core center, the core is stable to radial oscillations.

Figure 3-35 shows the power distribution taken along the $0^{\circ}-180^{\circ}$ axis 28 hours after the perturbation for Cases $i$ through iv. Note the dependence of the oscillation magnitude on the location of the perturbation.

The above results, together with the earlier results comparing the power oscillations following perturbations located at different azimuthal positions, show that for this particular core (11-foot, flat power distribution), with 


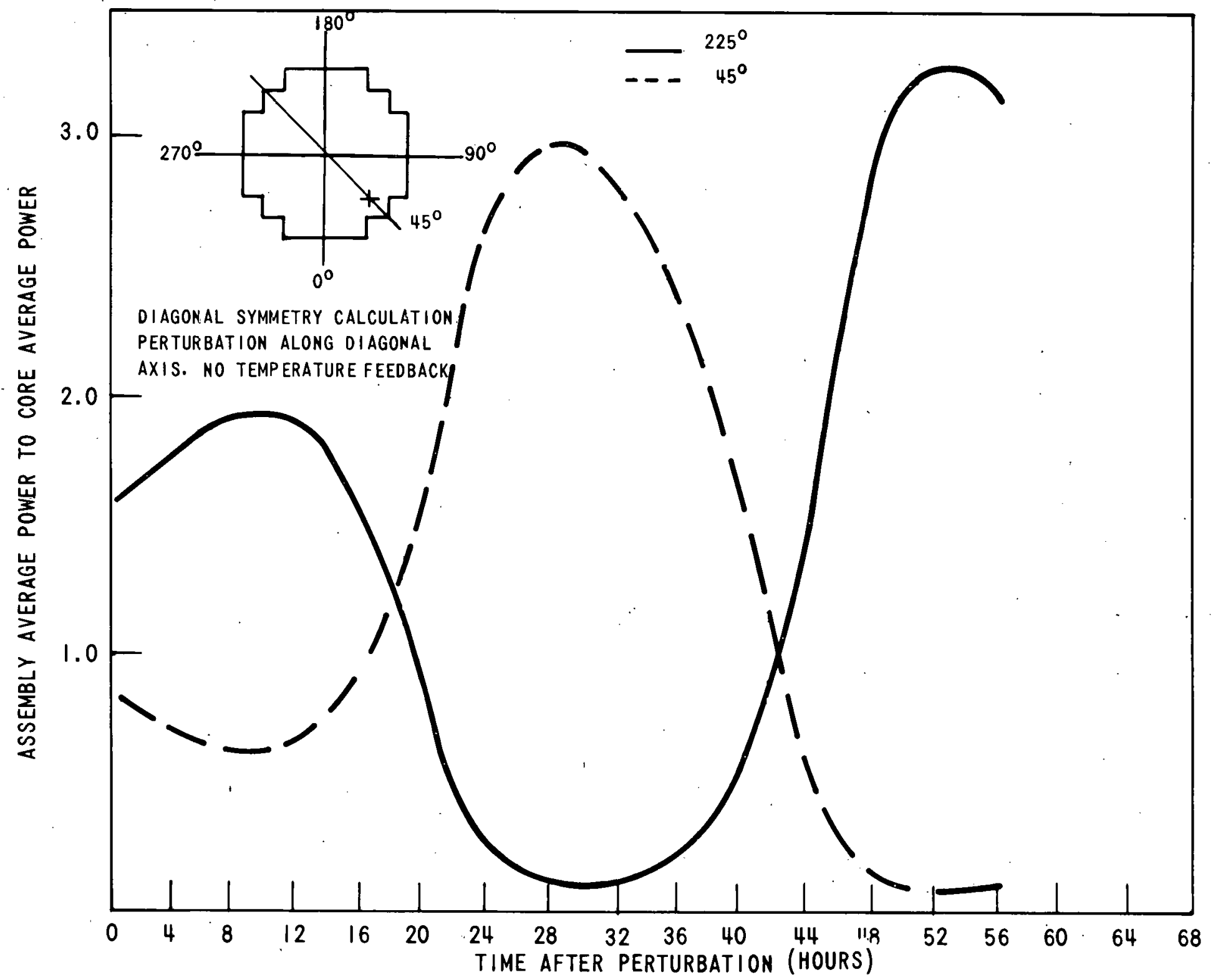

Figure 3-27. Xenon-Induced Oscillations along a $45^{\circ}-225^{\circ}$ Axis 


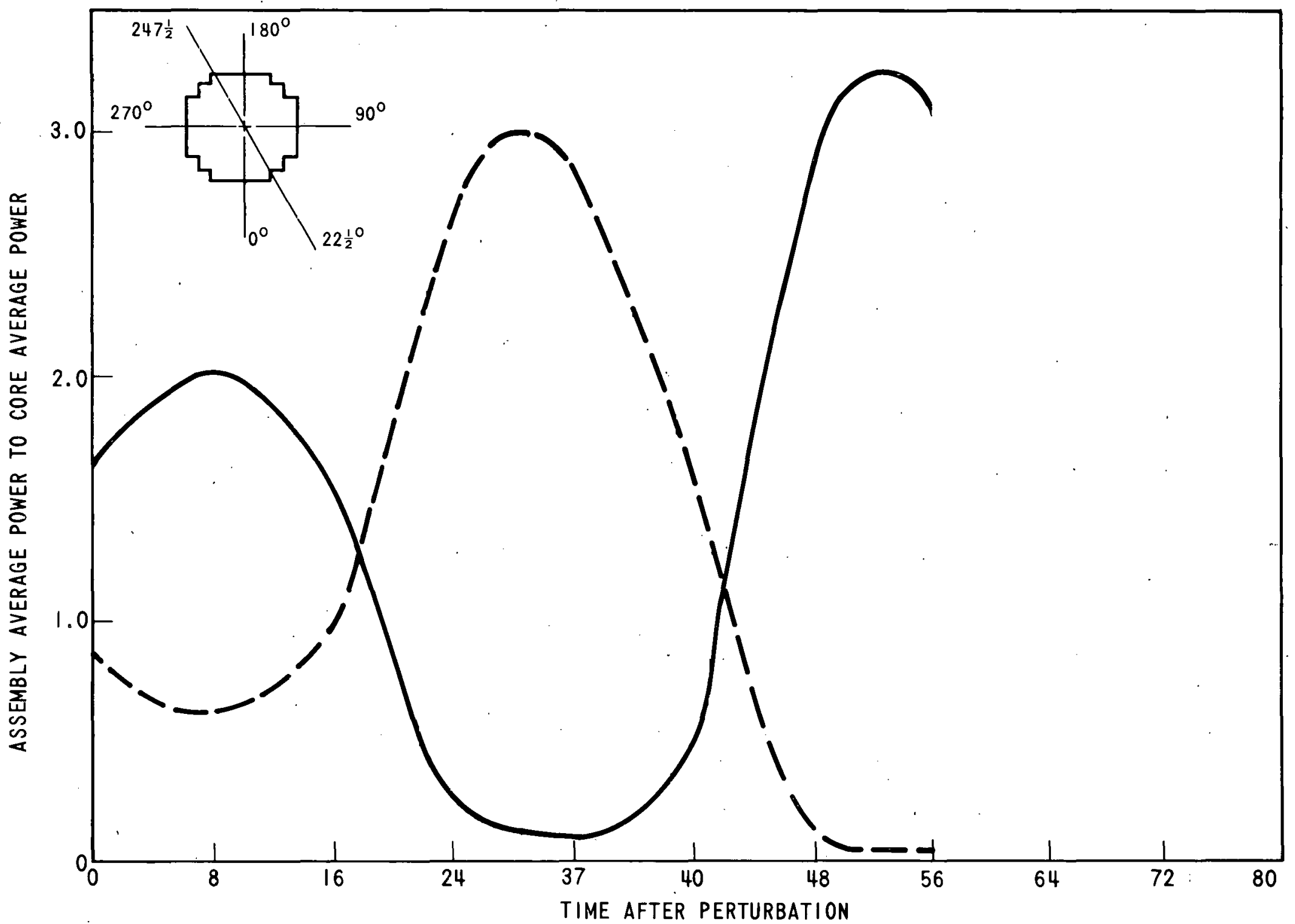

Figure 3-28. Xenon-Induced Oscillations along a $22-1 / 2^{\circ}-2471 / 2^{\circ}$ Axis 


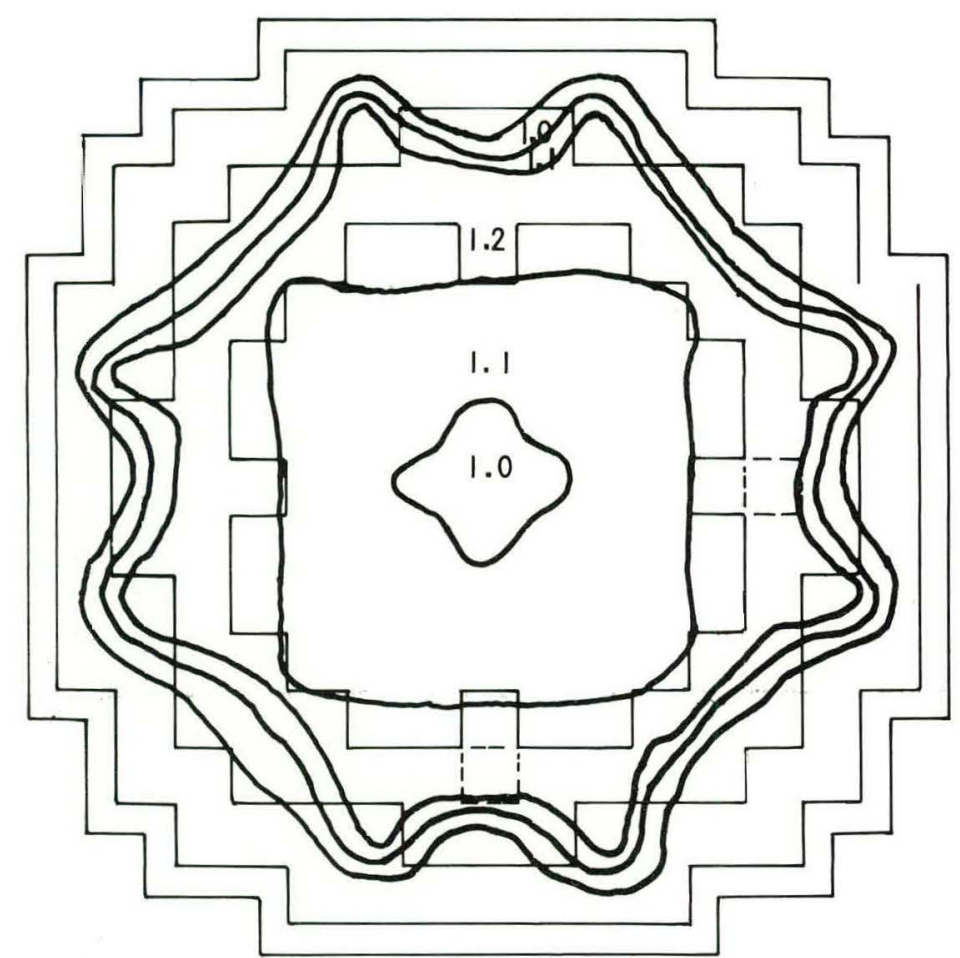

Figure 3-29. Contour Power Plot of Xenon Oscillations--Steady-State Flux Distribution for Full Core Calculation

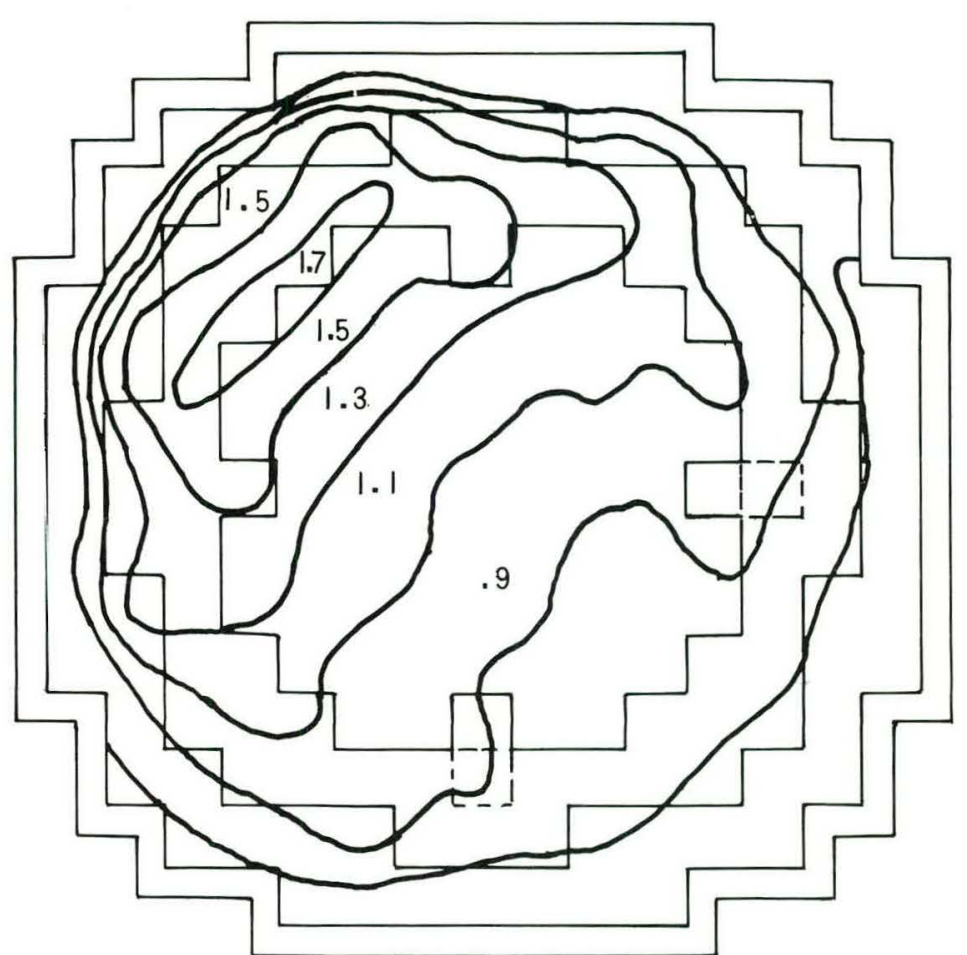

Figure 3-30 Contour Power Plot of Xenon Oscillations-Power Distribution 4 Hours after Removal of Perturbations

(Perturbation Positions shown by Dotted Squares) 


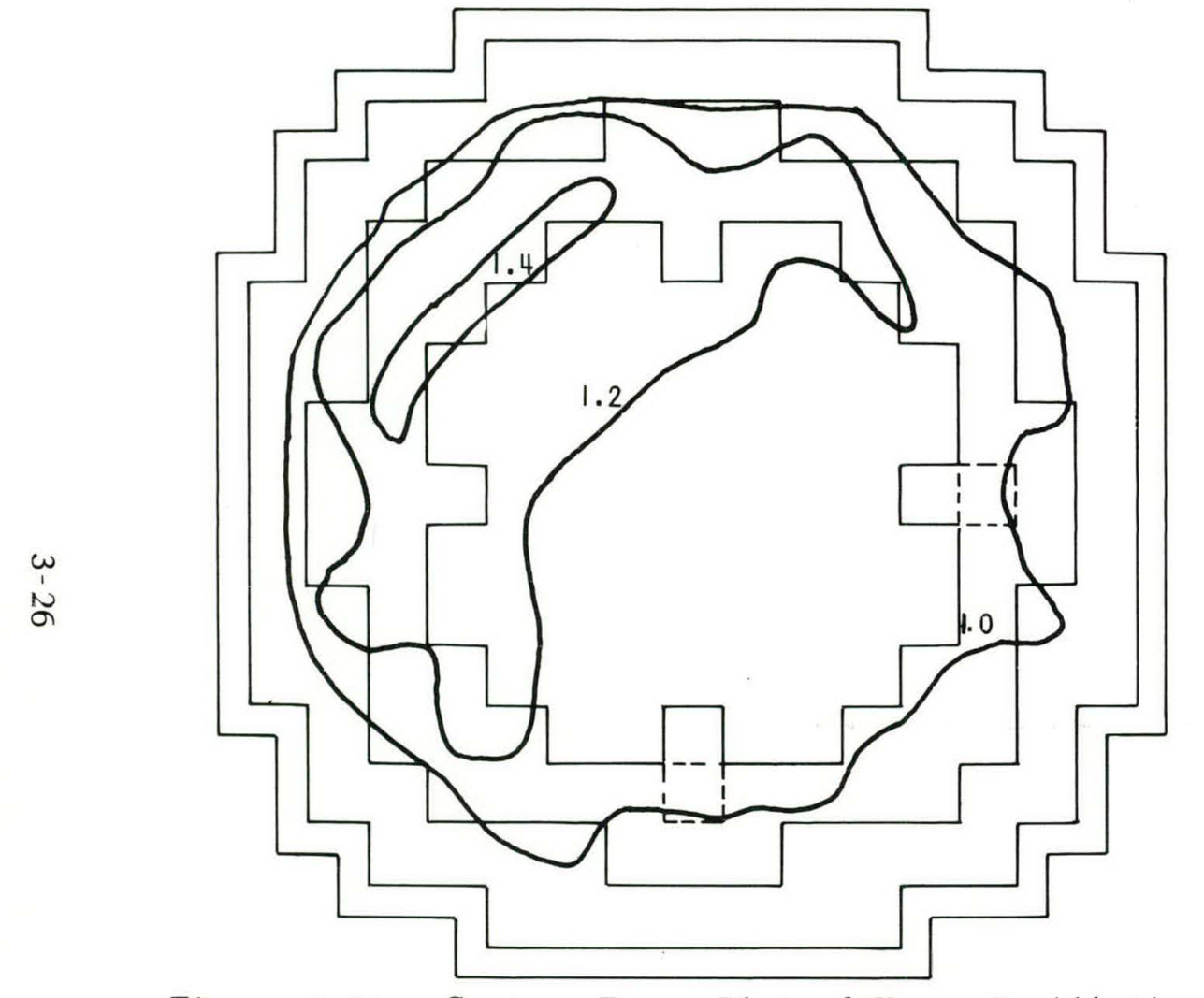

Figure 3-31 Contour Power Plot of Xenon Oscillations -. Power Distribution 12 Hours after Removal of Perturbation

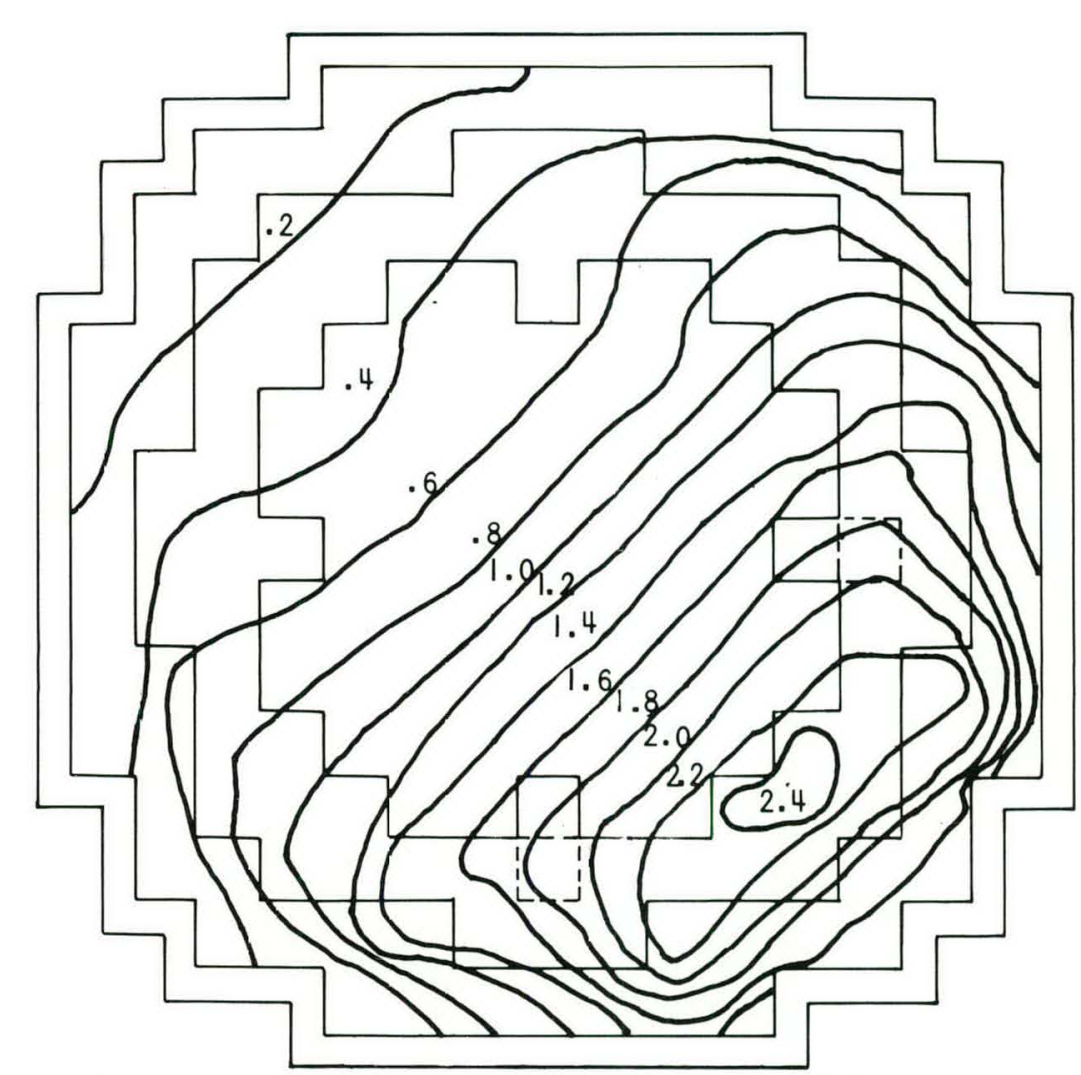

Figure 3-32. Contour Power Plot of Xenon Oscillations -- Power Distribution 24 Hours after Removal of Perturbations

(Perturbation Positions shown by Dotted Squares) 


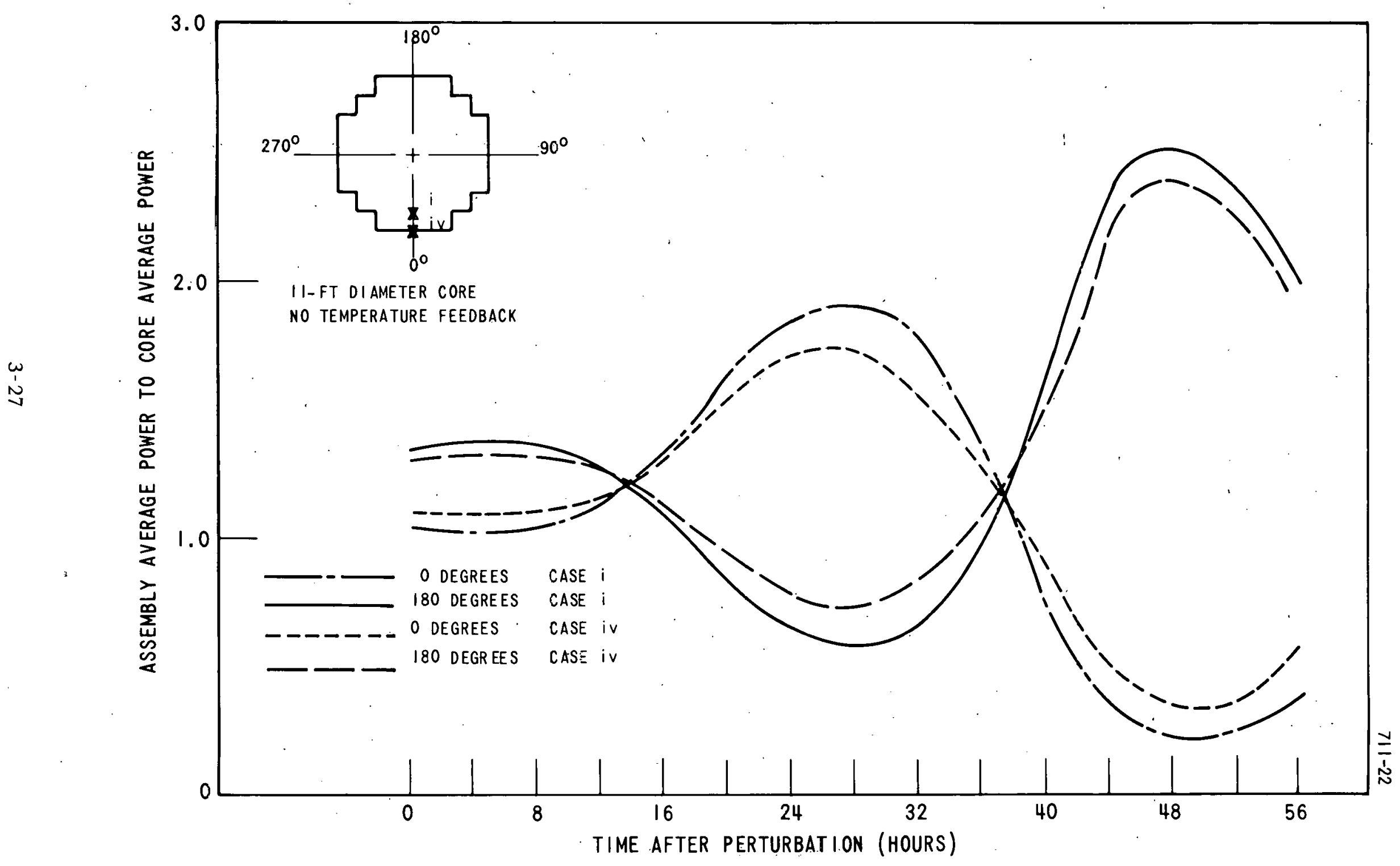

Figure 3-33. Effect of Perturbation Location on Xenon-Induced Oscillations (Cases $i$ and iv) 


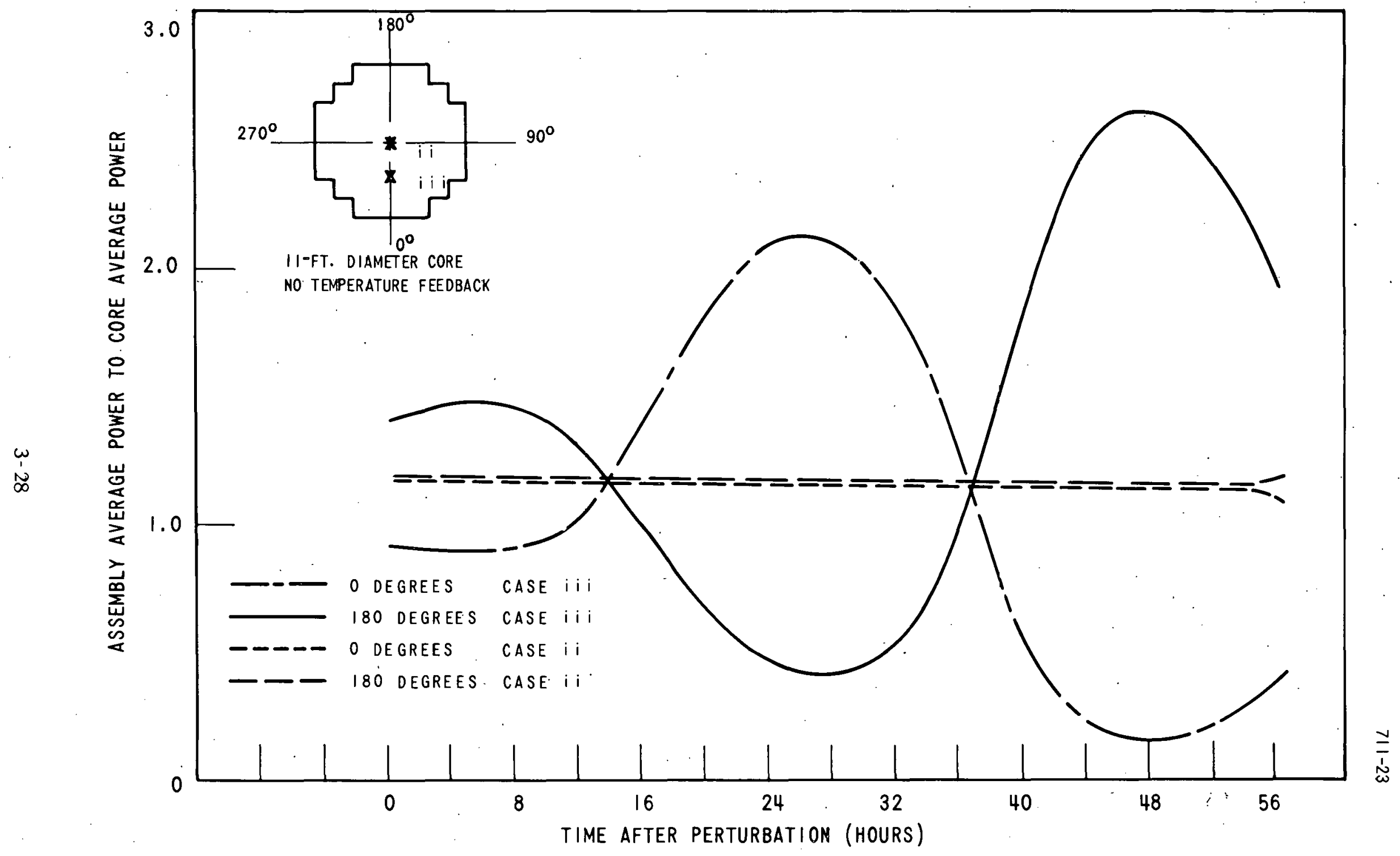

Figure 3-34. Effect of Perturbation Location of Xenon-Induced Oscillations (Cases i and ii i) 


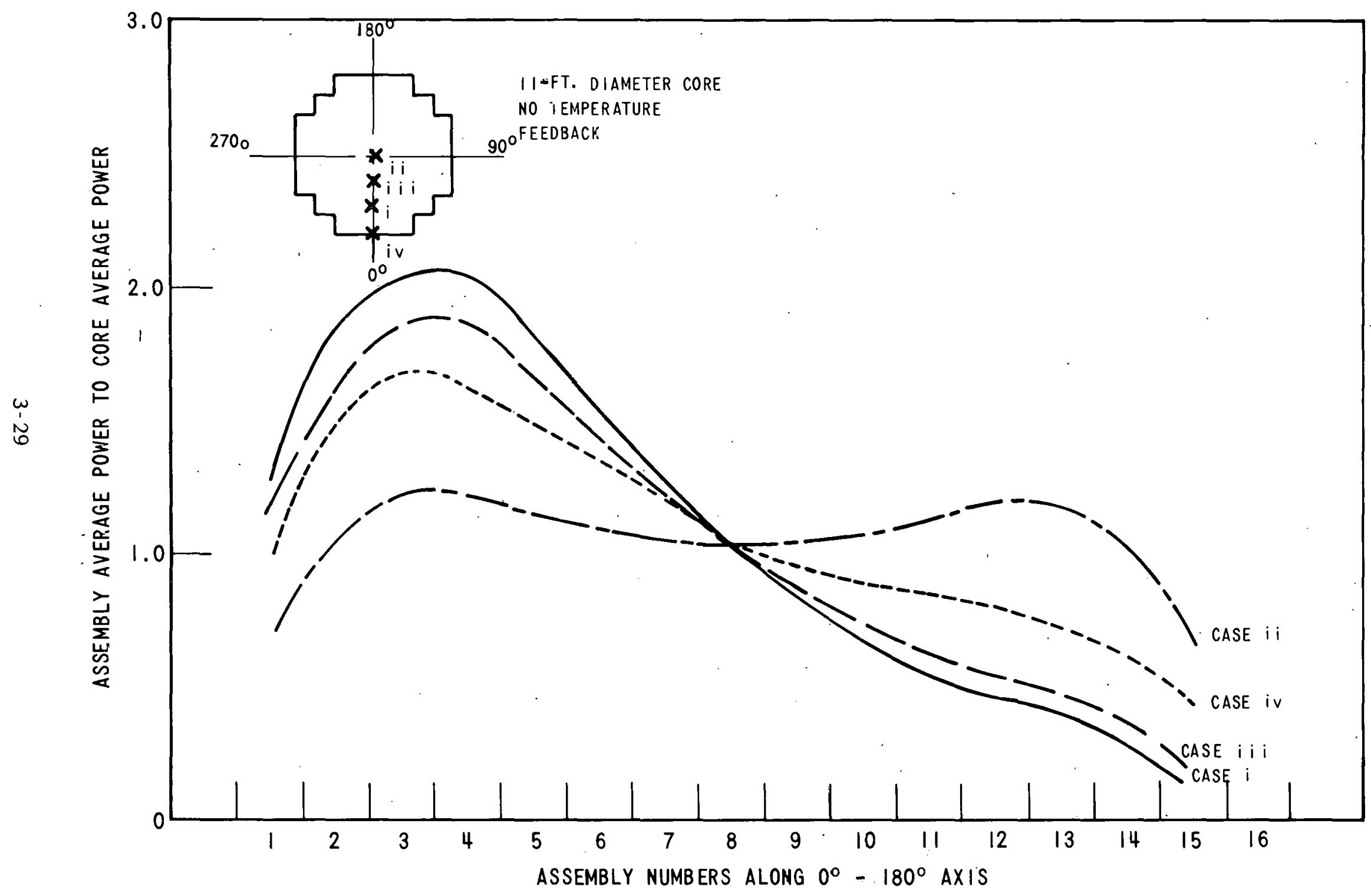

Figure 3-35. Effect of Perturbation Location on Power Distribution, 28 Hours after Perturbation 
the perturbation inserted for only a short time, xenon-induced azimuthal oscillations can be described in terms of essentially the same spatial mode, independent of the location of the perturbation.

Finally, an attempt was made to induce the oscillations to precess around the core. Since existing control systems are radially symmetric, it would not be possible to produce precessional oscillations in the manner described below. It was thought desirable, however, to determine if, under unusual circumstances (e.g., a jammed control rod) they could be excited at all, since the possibility of such oscillations is important for detection and control considerations.

The approach taken was to insert two poisons which were out of phase in time and at right angles to each other in space. One perturbation was introduced in the 11-foot core for an hour on one of the major axes. Sixteen hours after its removal the second was introduced for one hour. This was sufficient to produce quite strong precessional oscillations, as can be seen in Figures $3-36$ to $3-41$.

In the above, precessing oscillations produced by two perturbations were discussed and, for that particular power level, the precession was shown as quite marked. It appears, however, that the tendency to precess is related to power level in that the greater the power level the more easily a core will go into a precessional mode. Figures $3-42$ and $3-43$ show how the peaks move around on 11-foot core for a nominal full power and half power, respectively. It can be seen that as the power is increased, there is a tendency for the peak to shift from a diametral mode of oscillation to one of precession, given the initial conditions under which precessional oscillations are possible. This effect will become more prominent as reactor power and size increase, and control procedures should be developed accordingly.

\subsection{INFLUENCE OF MAGNITUDE OF THE PERTURBATION}

Both ZEST and TURBO* calculations were performed to ascertain the effect of perturbation strength on the oscillations. 


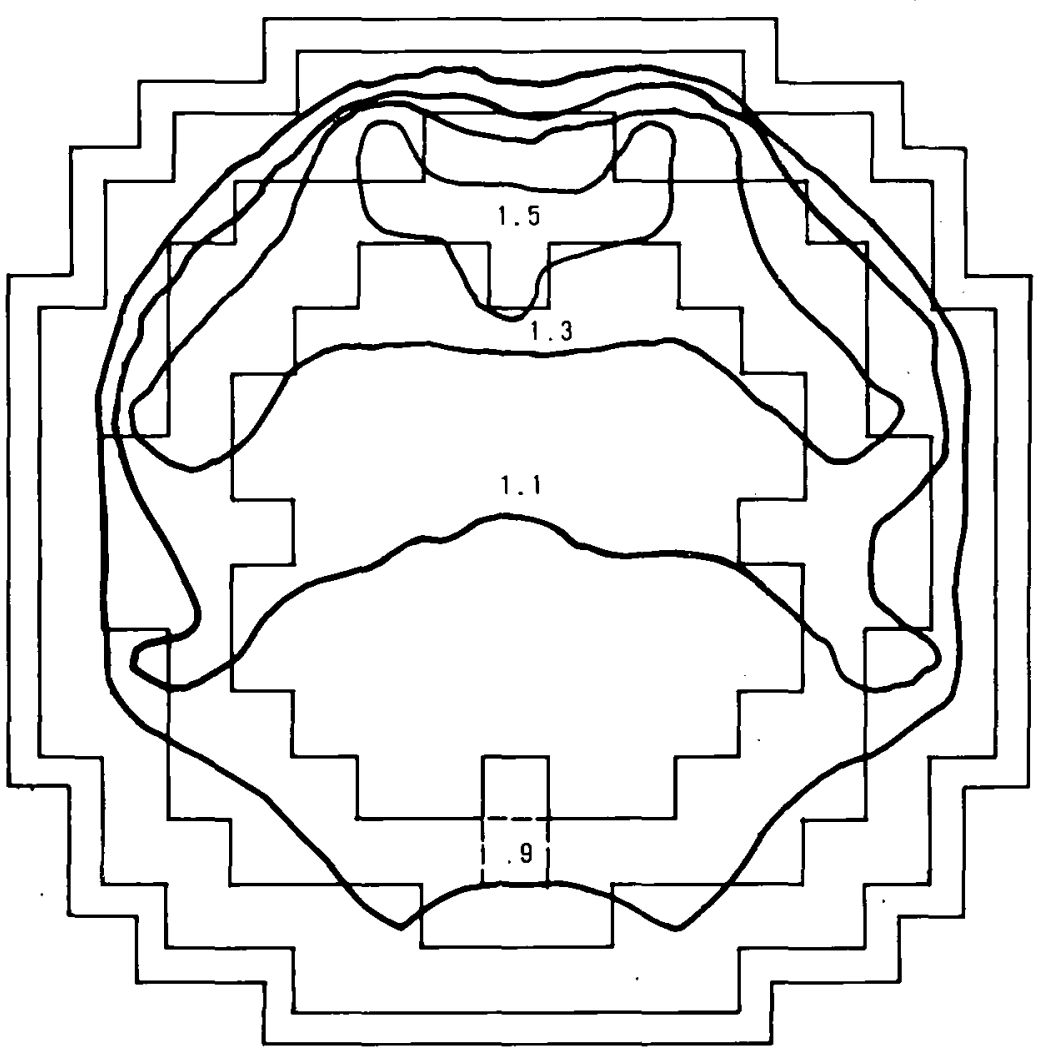

Figure 3-36. Contour Power Plot for Xenon-Induced Oscillations after Removal of First Perturbation

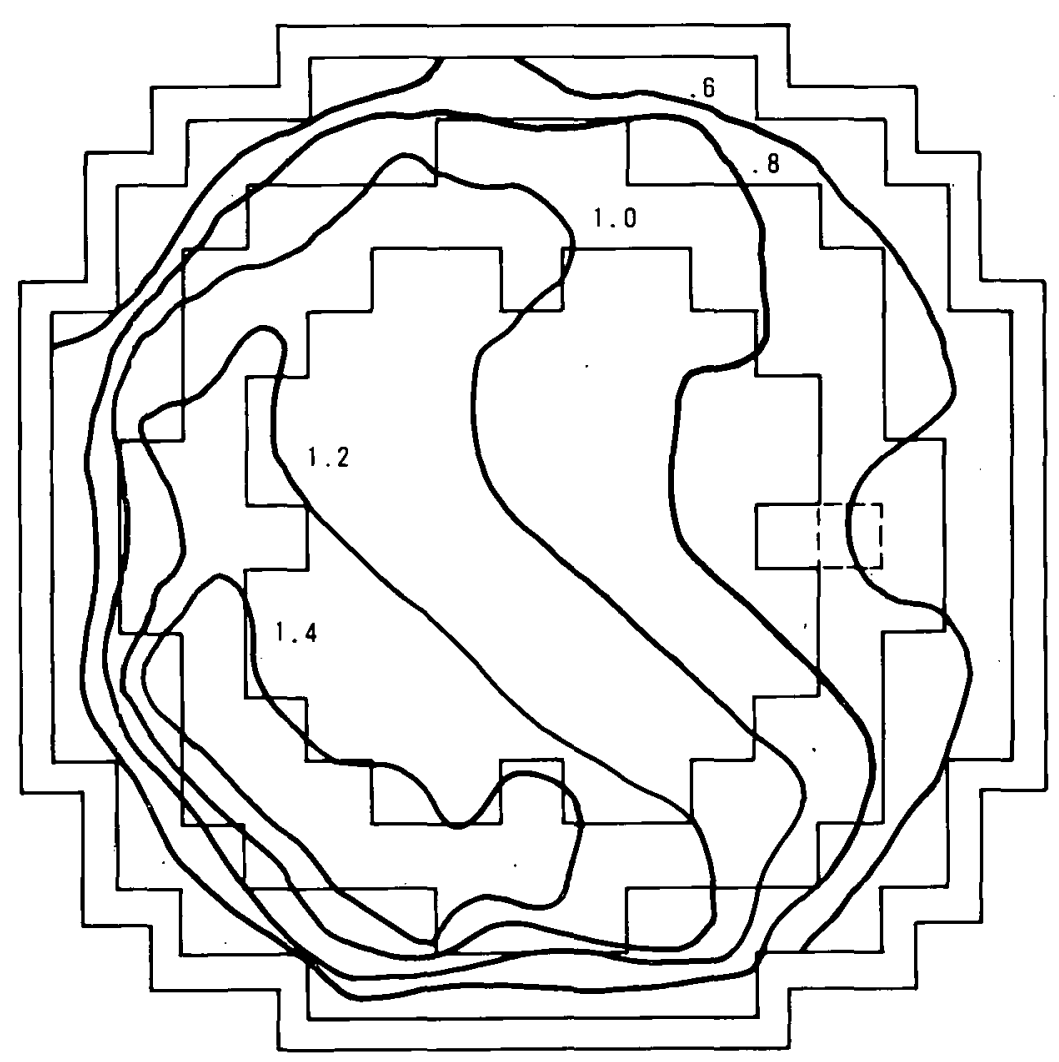

Figure 3-37. Contour Power Plot for Xenon-Induced Oscillations after Removal of Second Perturbation 


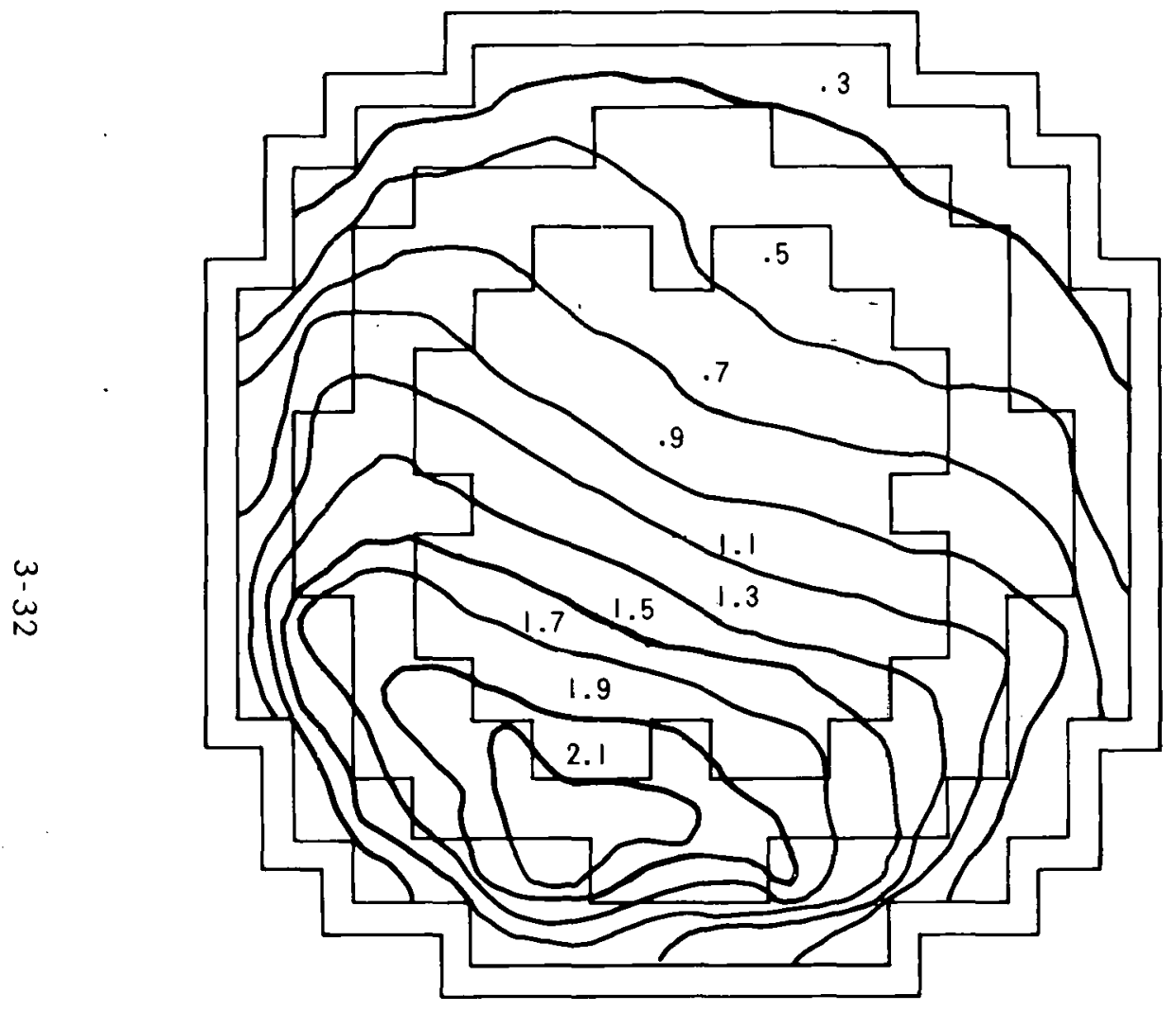

Figure 3-38. Contour Power Plot for Xenon-Induced Oscillations, 12 Hours after Removal of Second Perturbation

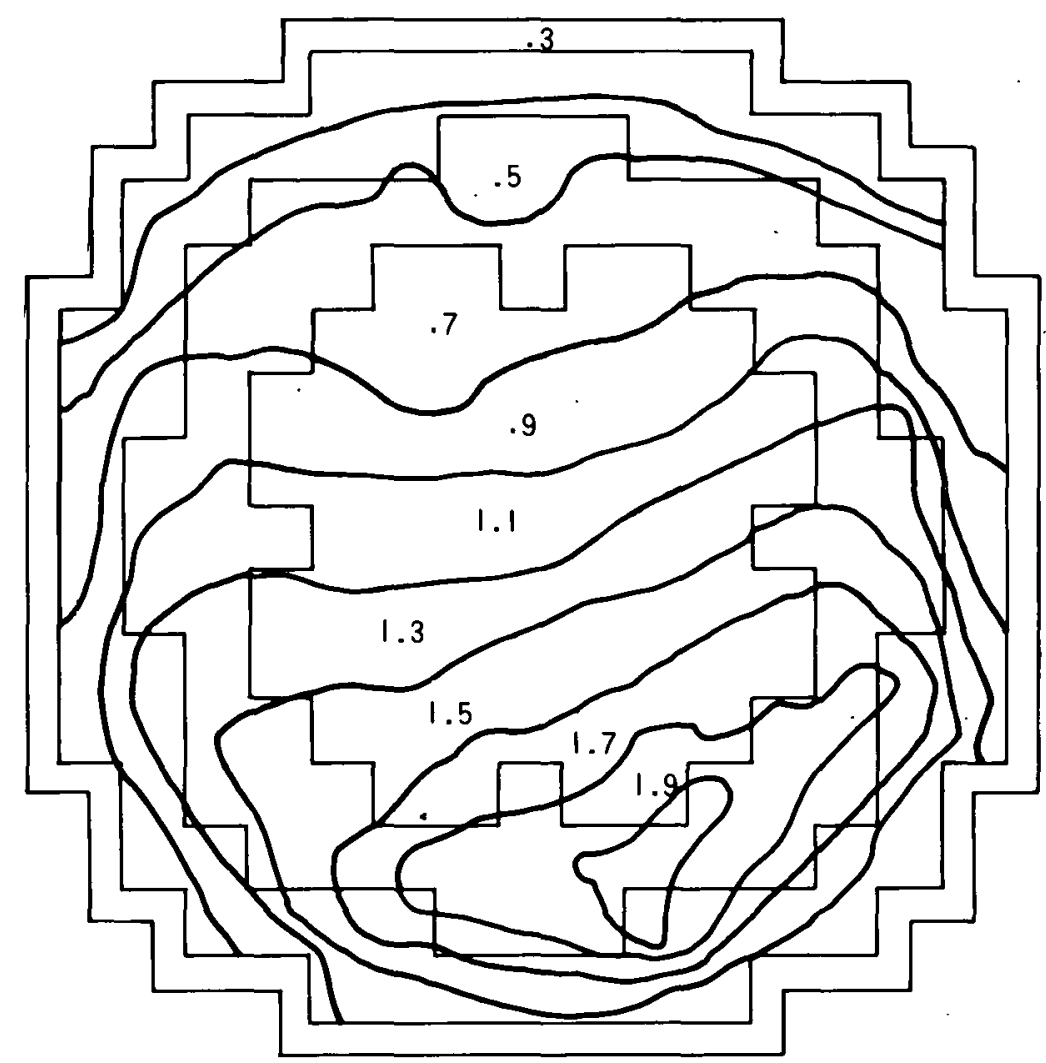

Figure 3-39. Contour Power Plot for Xenon-Induced Oscillations, 20 Hours after Removal of Second Perturbation 


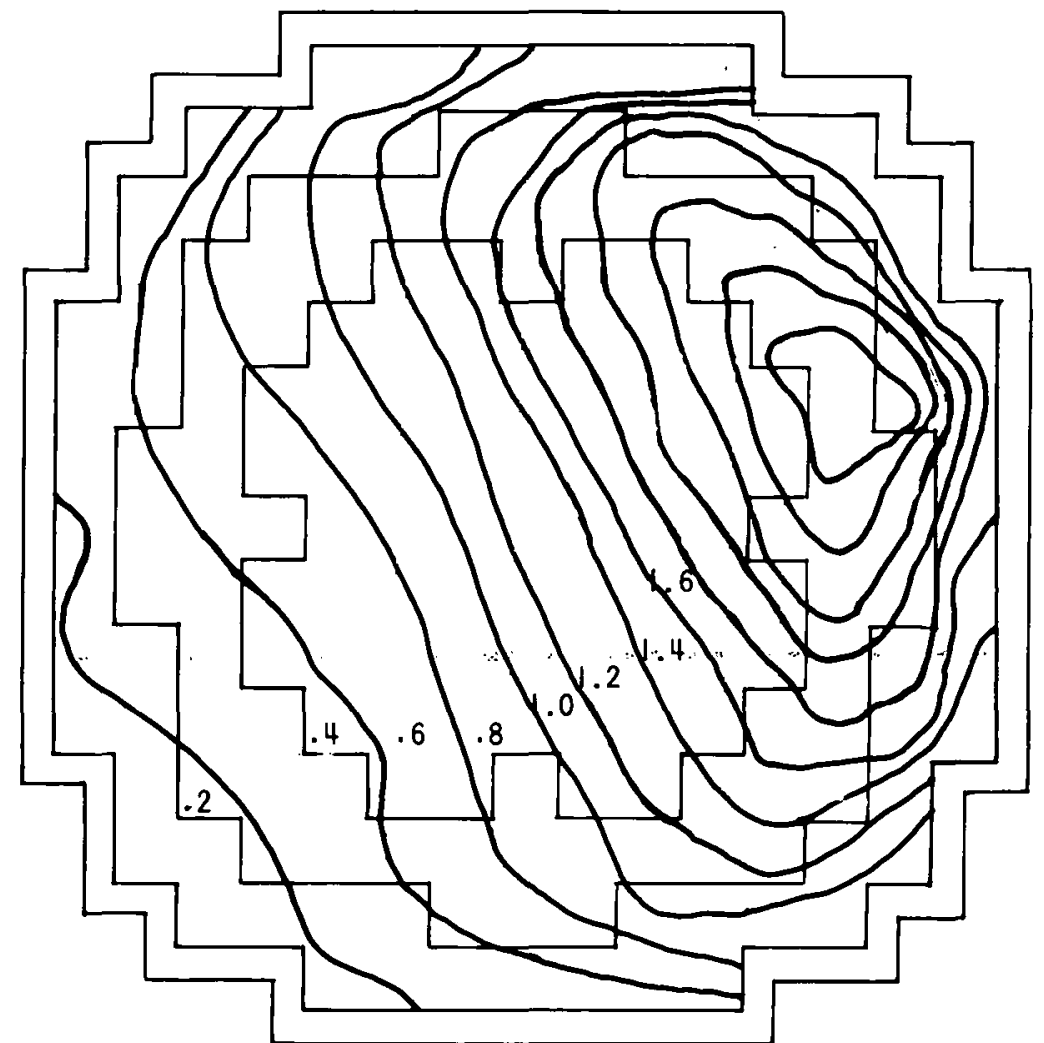

Figure 3-40. Contour-Power Plot for Xenon-Induced Oscillations, 28 Hours after Removal of Second Perturbation

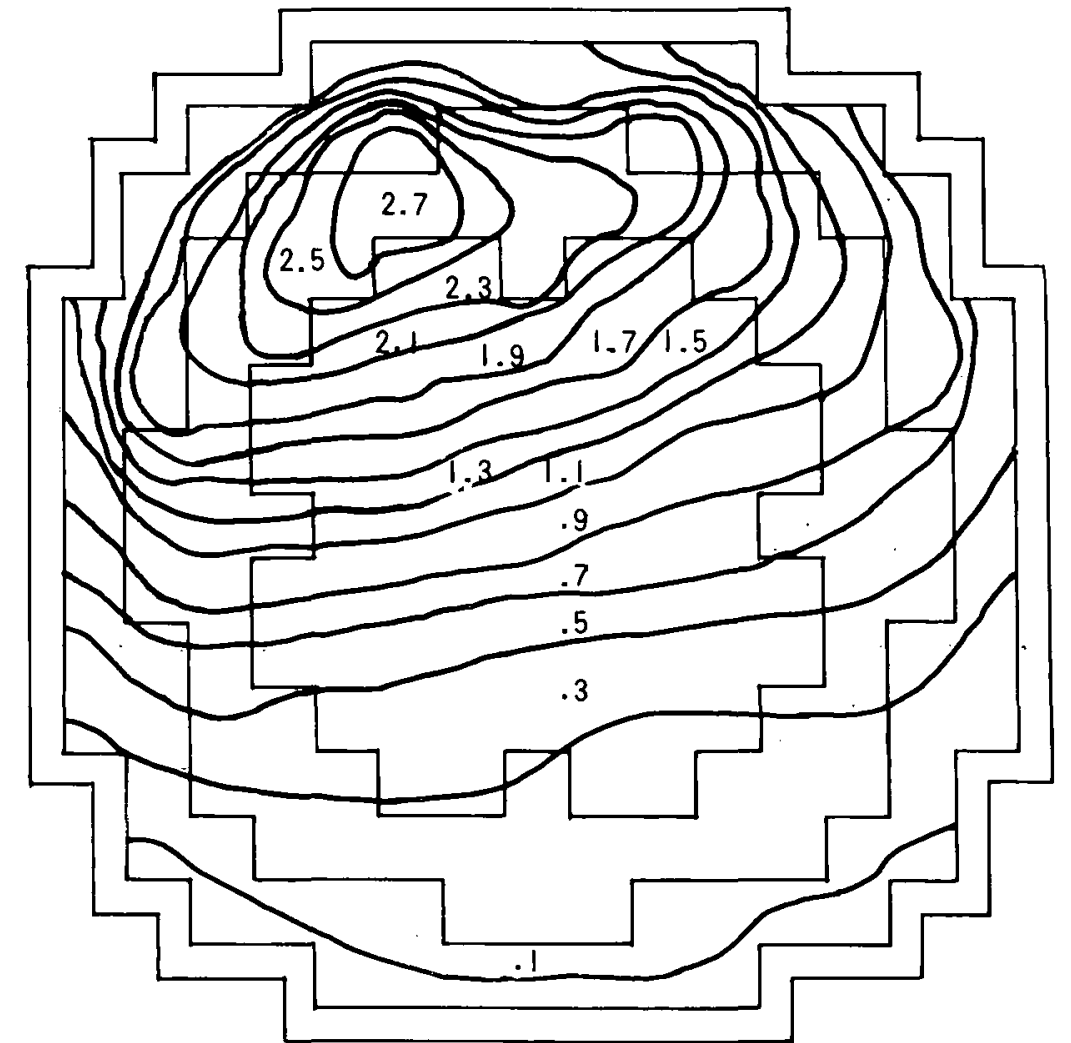

Figure 3-41. Contour Power Plot for Xenon-Induced Oscillations, 40 Hours after Removal of Second Perturbation 


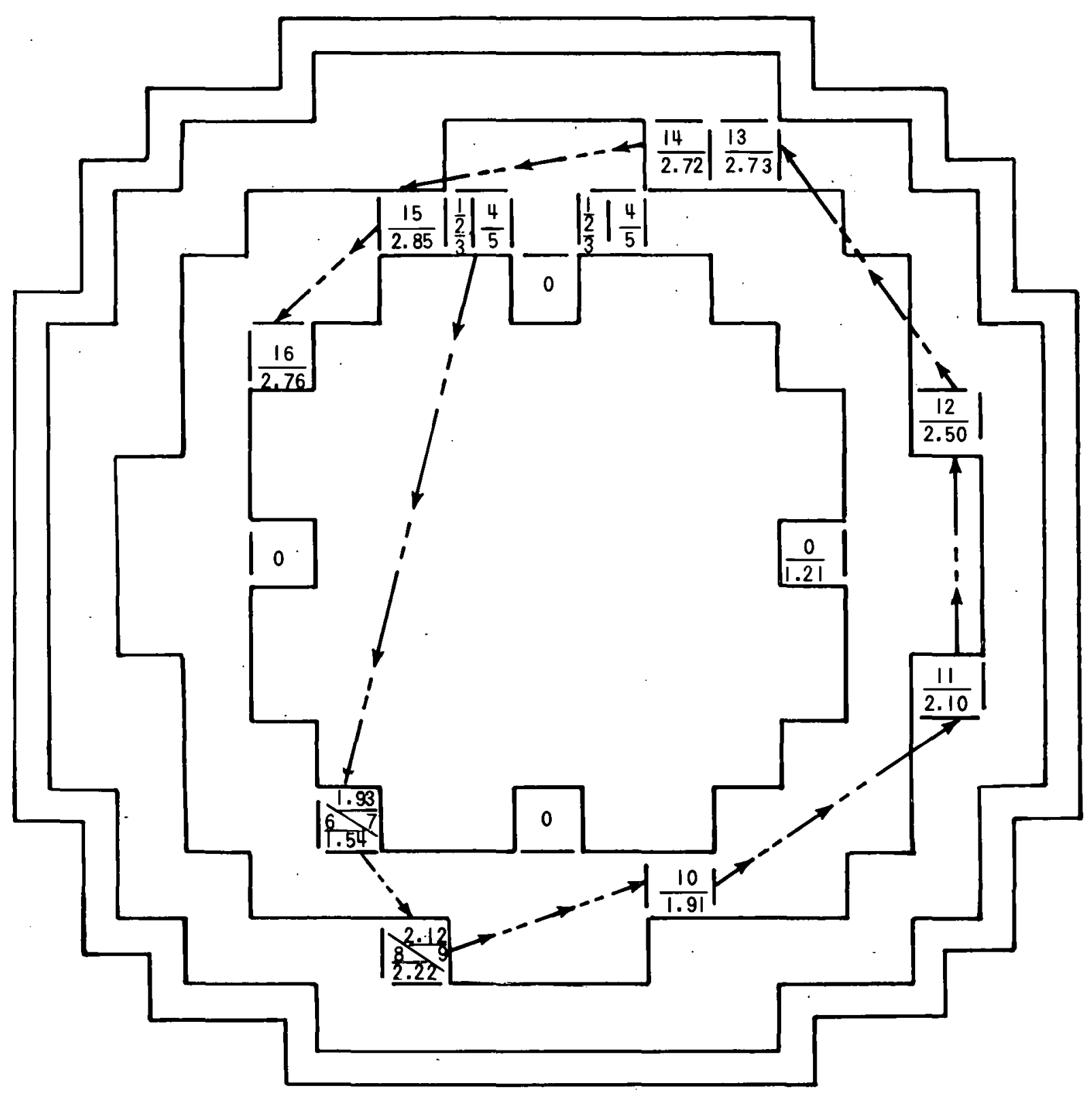

Figure 3-42. Precession of Xenon-Induced Power Peak in Full Core at Full Pówer 


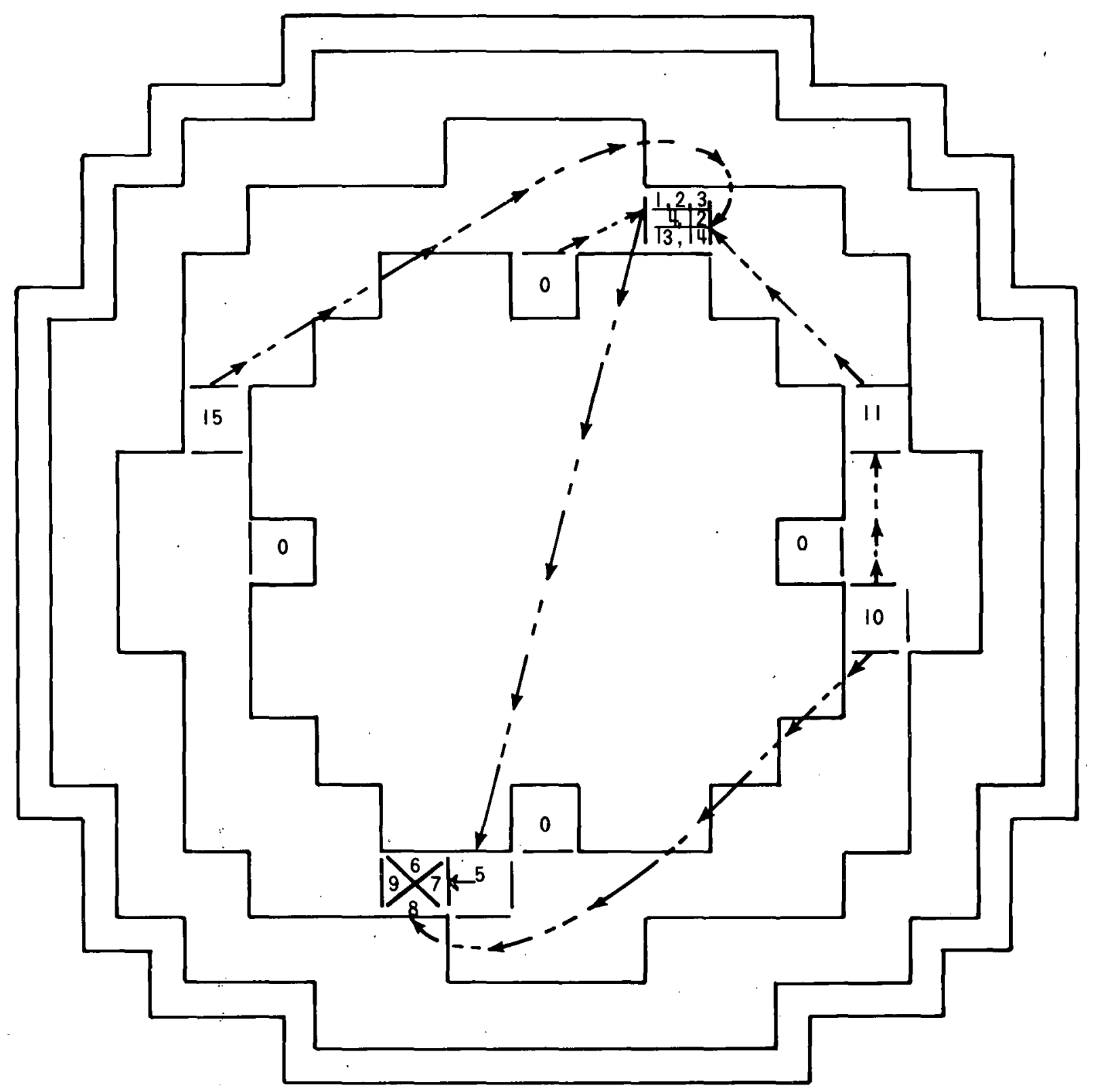

Figure 3-43. Precession of Xenon-Induced Power Peak in Full Core at Half Power 
With the size of perturbation used in most of the TURBO* calculations, the oscillations diverged quite rapidly and soon were in the non-linear range. In order to evaluate the sensitivity in the $x-y$ plane of the core to perturbation size, three TURBO* calculations were performed which had poisons that were reduced in strength to $1 / 2,1 / 5$ and $1 / 10$ of the original. The reactivity worth equivalents for these perturbations were $6 \times 10^{-4}$, $3 \times 10^{-4}$, and $1 \times 10^{-4}$, respectively -- that for the full perturbation being $9 \times 10^{-4}$ The results of these calculations were also useful in determining a suitable poison to produce linear oscillations in the investigation of certain parameters.

From the results shown in Figures $3-1,3-44$ and $3-45$ it can be seen that: 1) the period is not greatly affected by perturbation size, and 2) it requires a reactivity worth of $1 \times 10^{-4}$ or less to keep the first few oscillations in the linear range.

The calculations performed with ZEST were made in order to determine: 1) if the stability index were affected to any extent by the perturbation size, and 2) if this were the case, to find the reasons for this apparent variation. The effect was found to be present and its explanation came from several different areas. A large percent of this variation, however, came not from any physical reason but from the approximations or interpretations in the numerical model.

The stability index is derived from modal theory and, to have any meaning, must be, in effect, the index of the first overtone. However, this is contaminated with higher modes which make its evaluation inaccurate. This can be partially overcome by calculating the stability index at the nodal point of the second overtone, effectively eliminating the error caused by this mode. The effect of higher overtones can most probably be ignored as they decay quite rapidly: An alternative approach would be to insert a sma11 perturbation and wait long enough until modes other than the first had effectively died out. This would probably take a disproportionate amount of time and, due to the effectiveness of the first method, was not tried. 


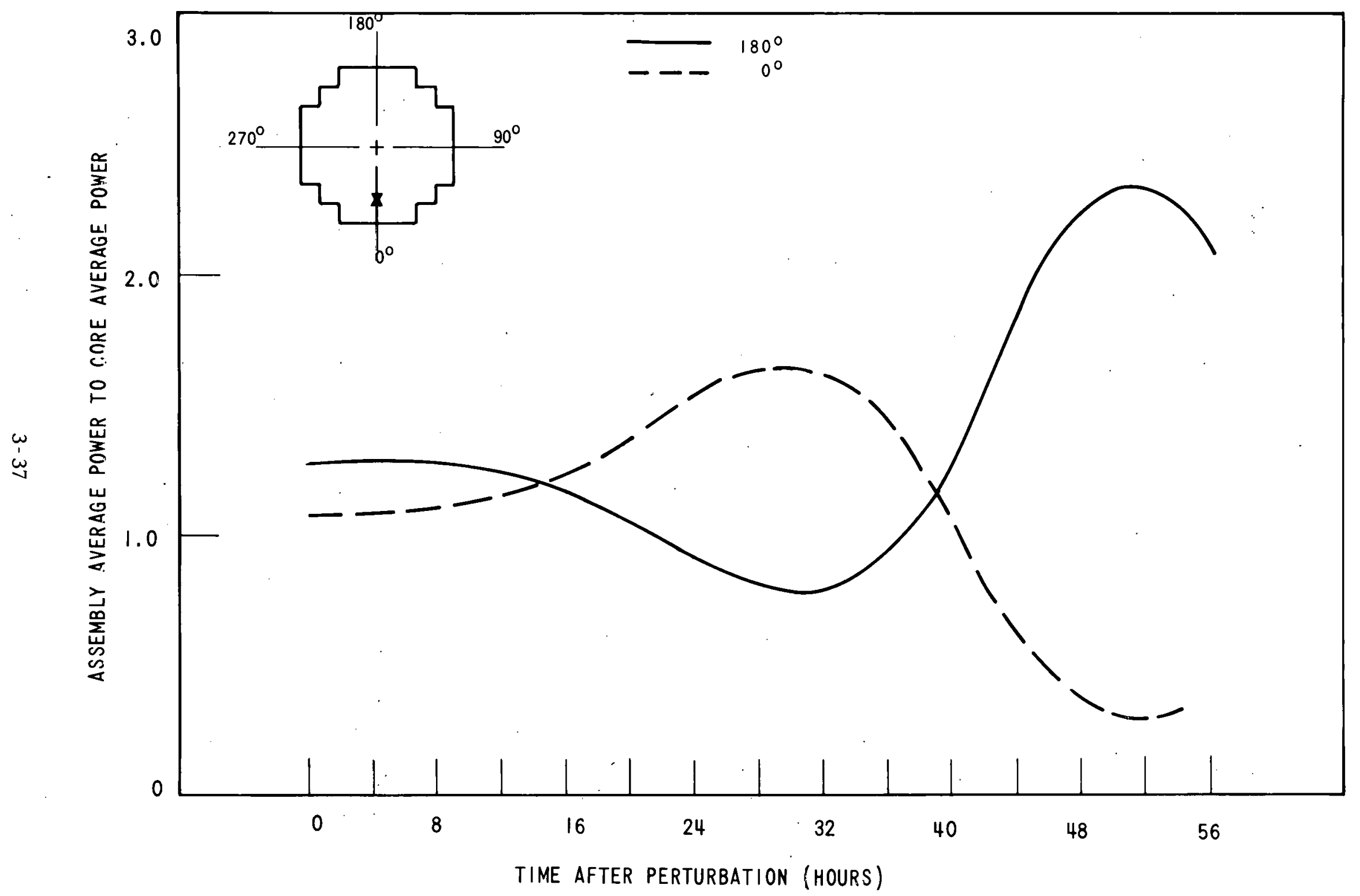

Figure 3-44. Xenon-Induced Oscillations for a Poison Reactivity Worth of $3 \times 10^{-4}$ 


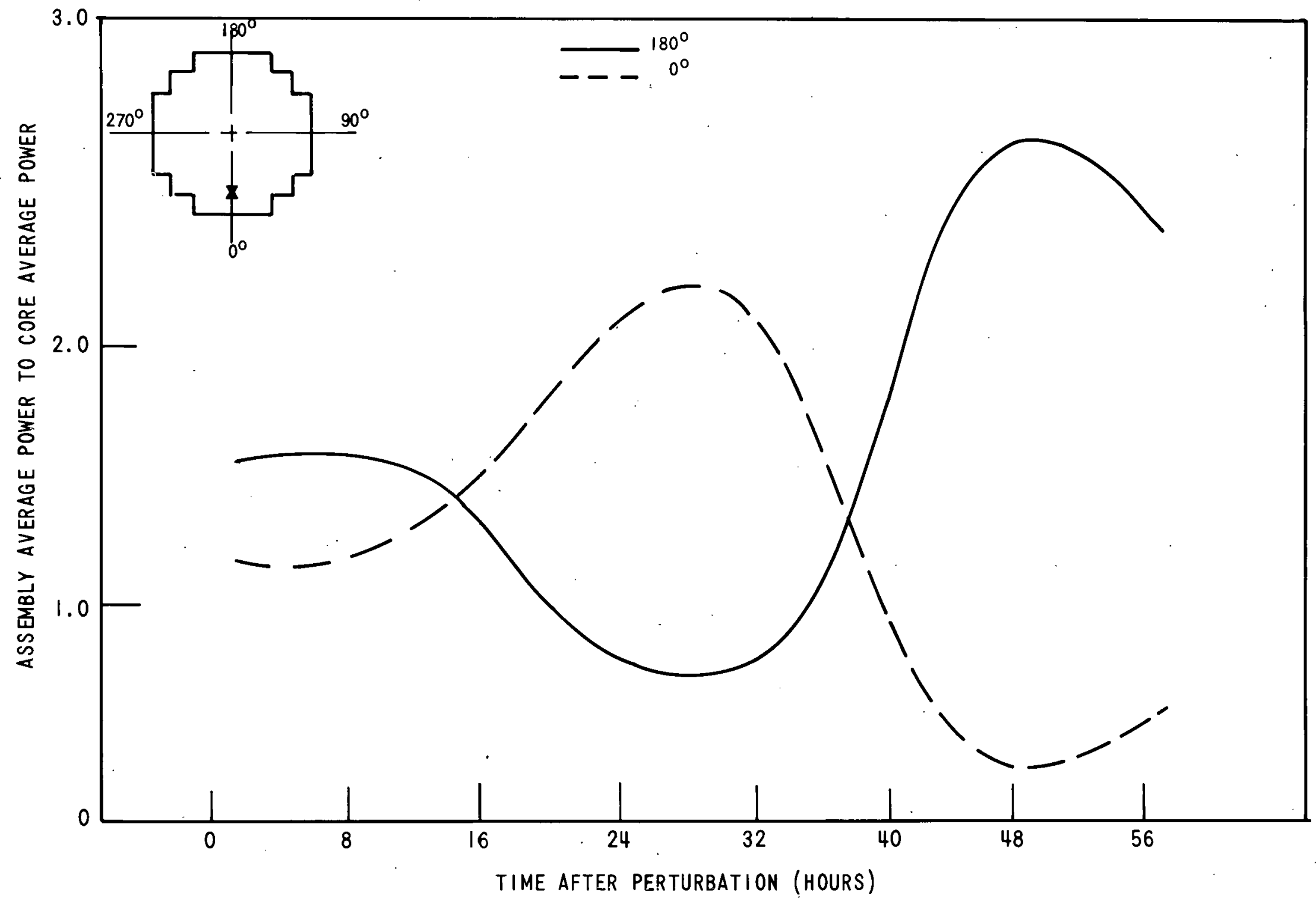

Figure 3-45. Xenon-Induced Oscillations for a Poison Reactivity Worth of $6 \times 10^{-4}$ 
Another source of error in the stability index was found to come from the interaction of the perturbation size and finite convergence criterion. As can be seen in Table 3-1, the stability index for a larger convergence criterion $\left(\varepsilon=10^{-4}\right.$ ) increases in size as the perturbation strength increases while for a tighter criterion $\left(\varepsilon=10^{-5}\right)$, the reverse trend is observed. A brief mathematical analysis of the effect of the convergence criterion and perturbation strength on the stability index was performed and it would seem that such an effect is indeed possible.

An investigation.was also carried out into the coupled effect of the fuel temperature feedback characteristics and the perturbation size on the stability index. In general, larger perturbations produce higher local flux levels and this, in turn, will lead to higher local fuel temperatures. Since the nonlinear characteristics of the fuel-temperature feedback lead to a decrease in the Doppler coefficient at higher temperatures, large osclllations should be effectively less stabilized than small oscillations. This effect would not be observed in a linear treatment of the fuel temperature feedback.

Of these three effects, the first two appeared to be the most important. Table 3-1 shows the stability indices for different perturbation sizes against fuel temperature and convergence criterion. These stability indices have been determined from the node of the second overtone and have been corrected for zero time-step length Section 2.3.2. In all cases the core under consideration was 11-foot in size and had a flattened power distribution. Two types of feedback were used. In the first, the fuel temperature was a linear function of power and, in this case, no effect of perturbation size should be observed. In the second, the proper mon-linear feedback was used. In case (3) in Table 3-1 it can be seen that the stability index is almost independent of perturbation size. This is because the convergence criterion has effectively been eliminated as a source of error [compare with case (2)]. Since the effect of the convergence criterion has been effectively eliminated in cases (3) and (4), the effect of the non-linear fuel temperature can be observed in these two cases. Both from cases 1 and 2 and cases 3 and 4, it can be seen that the non-linear nature of the fuel temperature feedback has, in this case, a negligible effect on stability. 
TABLE 3-1

CORRECTED STABILITY INDICES

Corrected Stability Indices For

\begin{tabular}{|c|c|c|c|c|c|}
\hline Case & Feedback & Convergence & $1 / 2$ Pert. & Nominal Pert. & $3 / 2$ Pert. \\
\hline 1 & non-linear & $10^{-4}$ & 0.01 & 0.017 & 0.020 \\
2 & linear & $10^{-4}$ & -- & 0.023 & 0.027 \\
3 & linear & $10^{-5}$ & -- & 0.029 & 0.028 \\
4 & non-linear & $10^{-5}$ & -- & 0.025 & 0.023 \\
\hline
\end{tabular}

\subsection{EFFECT OF VERY SMALL PERTURBATIONS ON CORE STABILITY}

The linearized modal expansion theory of spatial xenon stability with linear feedback predicts no threshold which is related to perturbation size. In other words, a core which is predicted to be unstable, no matter how close to the stability threshold, will undergo divergent (if small) flux oscillations following any perturbation, regardless of how small in magnitude. In an operating reactor, the presence of inherent fluctuations in the neutron flux (noise) may somewhat alter this conclusion. Indeed, an input perturbation of a magnitude smaller than the magnitude of such fluctuations would not be expected to cause any change in the behavior of the system.

A series of one-dimensional ZEST calculations was performed in an effort to obtain some quantitative results related to the effect of very small perturbations. In a digital simulation with a diffusion theory code, pseudofluctuations or a pseudo-noise field are introduced by round-off errors in the machine as well as by the nature of the eigenvalue calculation itself, which is usually terminated when the pointwise fluxes are converged to within a criterion $\varepsilon$. The use of a non-zero time-step also introduces errors in the calculations, therefore causes pseudo-flux fluctuations. This last effect was discussed in Section 3.2.

A 12-foot uniform slab with an average enrichment of 2.6 a/o and operated at an average power density of $84.5 \mathrm{w} / \mathrm{cc}$ was selected for analysis. Temperature feedback was neglected. This core, as shown on Figure 3-46, is spatially 


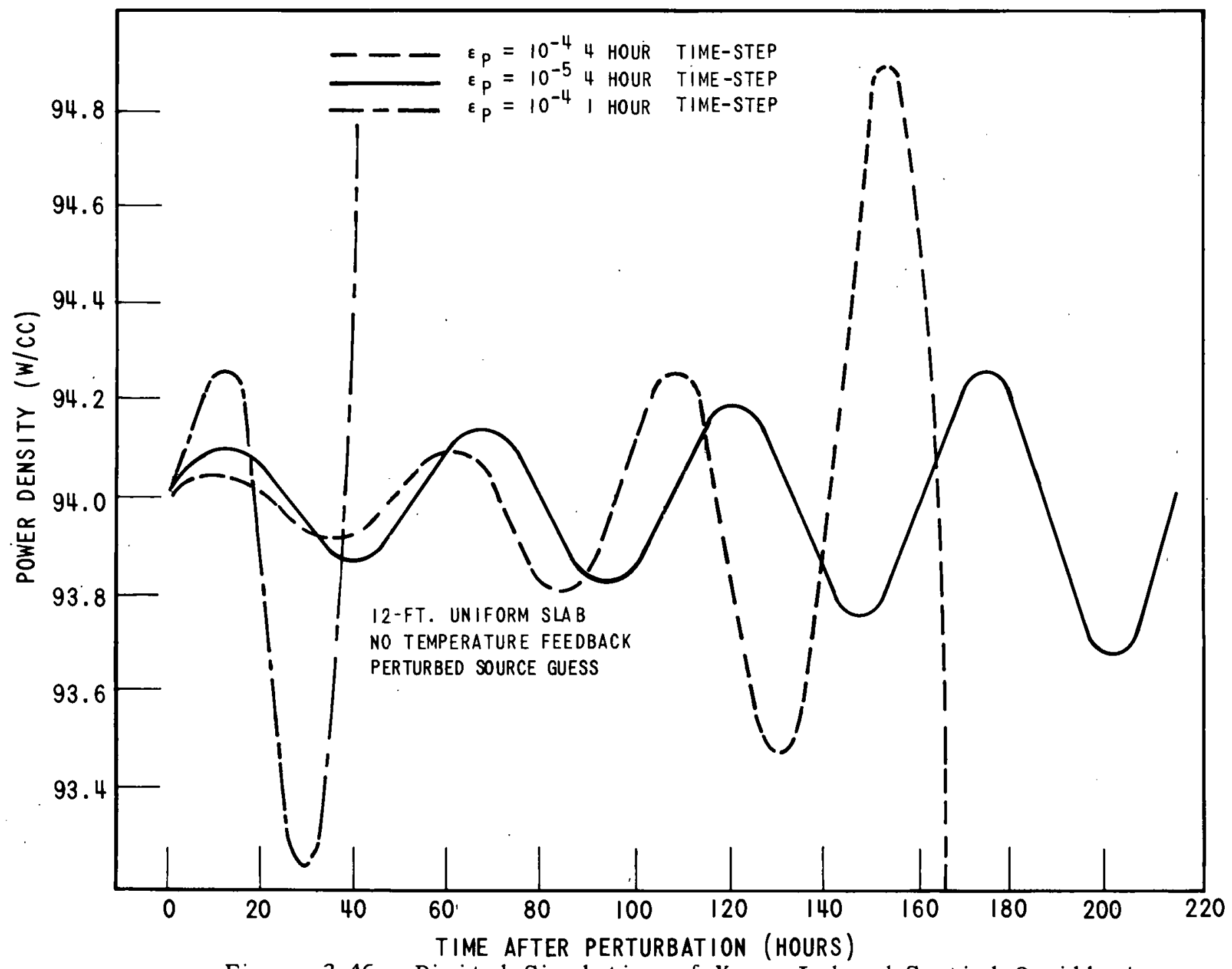

Figure 3-46. Digital Simulation of Xenon-Induced Spatial Oscillations 
unstable. A first series of calculations was performed for which the following procedure was followed.

Following the establishment of equilibrium conditions, a perturbation of zero magnitude was inserted into the core. In the eigenvalue calculations of the flux distribution following insertion of the perturbation, and in subsequent time-steps, the source guess, which normally would be the converged source distribution from the previous eigenvalue calculation (Appendix D), was uniformly perturbed by about 3 percent. The new converged flux distribution was thus somewhat different from the initial eigenvalue calculation, although within the convergence criterion $\varepsilon$. This difference in converged flux distribution can be seen on Figure 3-46. Following the removal of the zero magnitude perturbation, the core was followed at regular intervals for a period of 220 hours. In so doing, different time-step lengths (two hours and one hour, respectively) and different values of the pointwise flux convergence criterion ( $\varepsilon=10^{-4}$ and $\varepsilon=10^{-5}$, respectively) were used. The results of these calculations are shown on Figure 3-46. In every case, divergent flux oscillations were excited, even though the size of the input perturbation was zero. Moreover, both the growth rate and the period of oscillations are very sensitive to the value of the convergence criterion and of the time-step length. Figure 3-47 shows, on an extended scale, the resulting oscillation for a time-step length of one hour and a convergence criterion of $10^{-4}$.

A second series of calculations was performed where the source guess for each eigenvalue calculation was not perturbed and thus corresponded to the converged-source distribution from the previous eigenvalue calculation. Again both time-step length and pointwise convergence criterion were varied. The results of the calculations are displayed on Figure 3-48. Since the source guess to the eigenvalue calculations was not perturbed, the pseudofluctuations arising from insertion of a zero perturbation in this set of calculations were much smaller in magnitude than for the calculations of Figure 3-46. Figure 3-48 shows that all calculations tend to converge to the same limit, apparently the true solution, and that with time-step lengths of one hour, the flux does display an oscillatory behavior -- although convergent. 


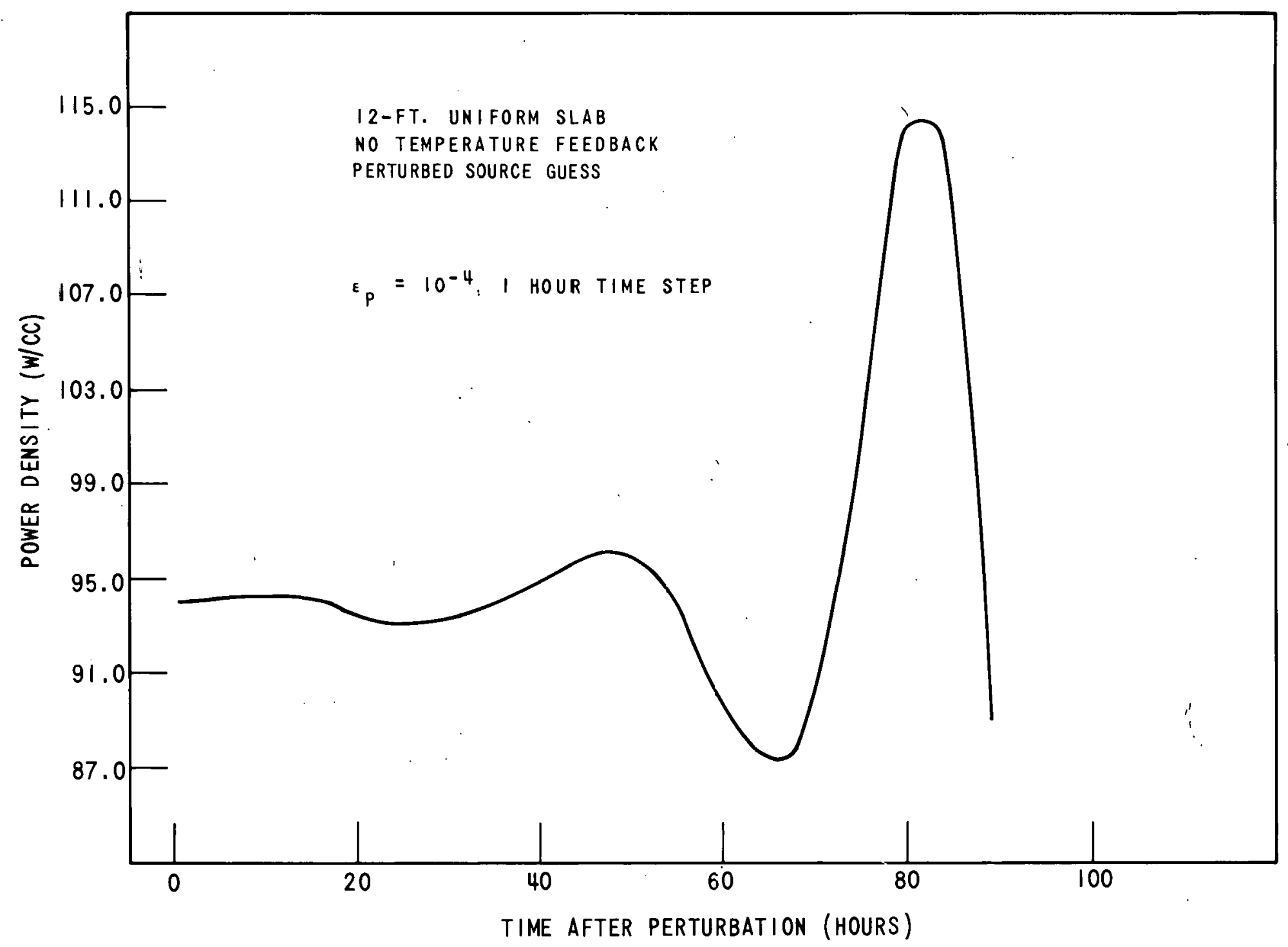

Figure 3-47. Digital Simulation of Xenon-Induced Spatial Oscillations with Very Smal1 Perturbation (Perturbed Source Guess :- Condensed Power Density Scale) 


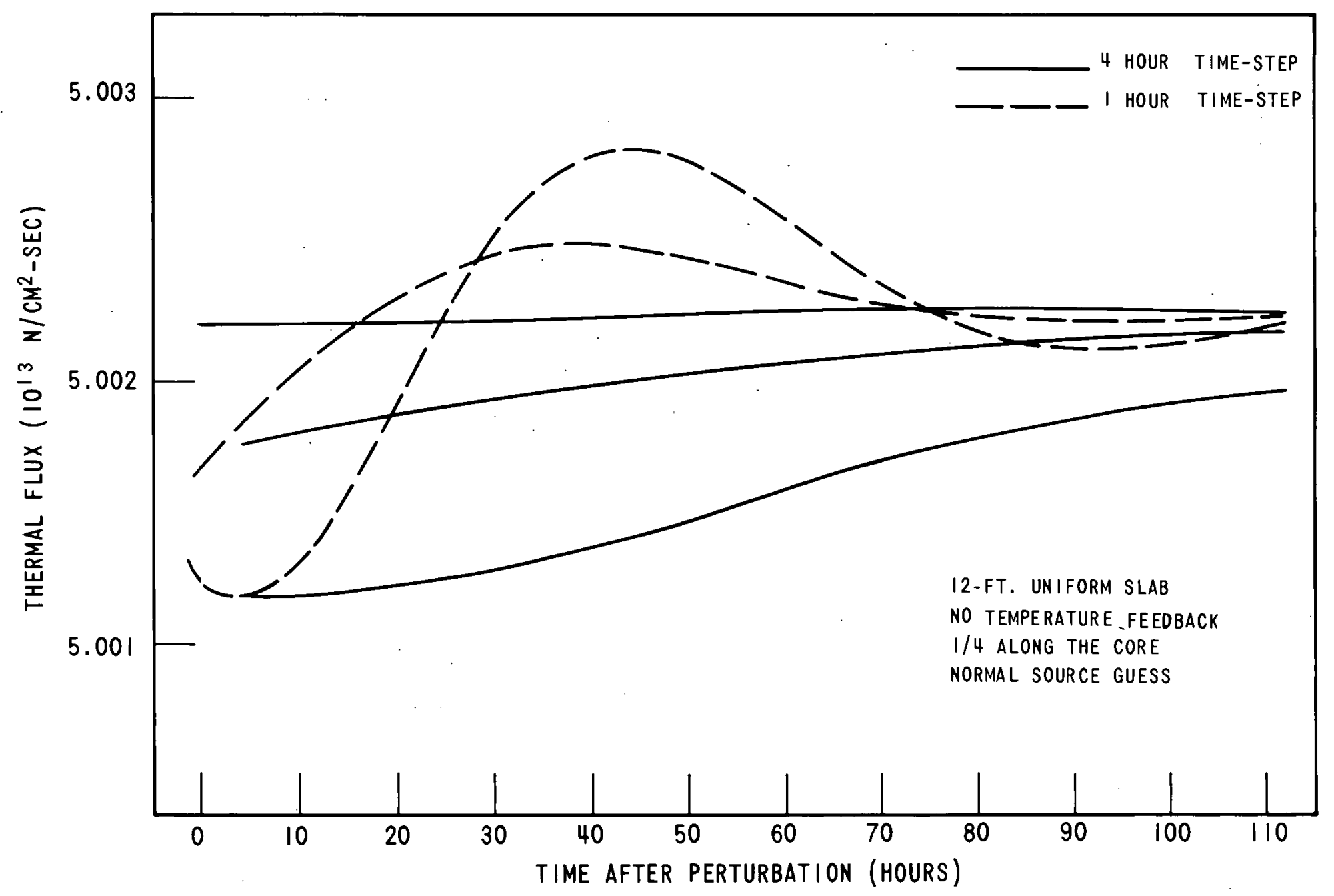

Figure 3-48. Digital Simulation of Xenon-Induced Spatial Oscillations with Very Small Perturbation (Normal Source Guess) 
From the limited number of numerical calculations described herein, it is possible to deduce at least one conclusion: namely, that very small perturbations of the flux in an otherwise unstable core can excite divergent oscillations. Moreover, it appears that the pseudo-fluctuations introduced by the use of a non-zero convergence criterion and a non-zero time-step length significantly affect the observed stability characteristics. It should be noted, however, that the numerical noise is unlikely to simulate the reactor noise accurately. This can be seen from the fact that the type of oscillations excited is very sensitive to the nature of the numerical noise introduced.

\subsection{EFFECT OF TEMPERATURE FEEDBACKS}

The effect of fuel and moderator temperature feedbacks was examined to determine the extent of their stabilizing effect on xenon oscillations. An analysis of only the Doppler feedback was made in one dimension with ZEST and in two dimensions with TURBO*. The effect of principally moderator feedback was examined qualitatively using SPARTA.

In the TURBO* calculations, an 11-foot core with an average enrichment of 2.6 percent was used. Two calculations with this core were run -- one with a flat equilibrium power distribution, and one considerably dished. The steady-state power distributions for these conditions are compared in Figure 3-49 and the subsequent oscillations in time are shown in Figures 3-50 and 3-51 (curves $B$ and $B^{\prime}$ ). These are shown against the equivalent curves when no Doppler feedback is present (curves $A$ and $A^{\prime}$ ). It can be seen that the stabilizing influence of the feedback is important, both in the fact that the initial transients following the perturbation are significantly reduced in magnitude, and that stability is considerably enhanced.

In interpreting the above results, the following important considerations must be borne in mind:

1. The TURBO* calculations were performed with a four-hour timestep. As was shown in Section 2.3 .2 a correction.for the finite time-step results in the core actually being unstable. The same comment applies to the results for the case with a 


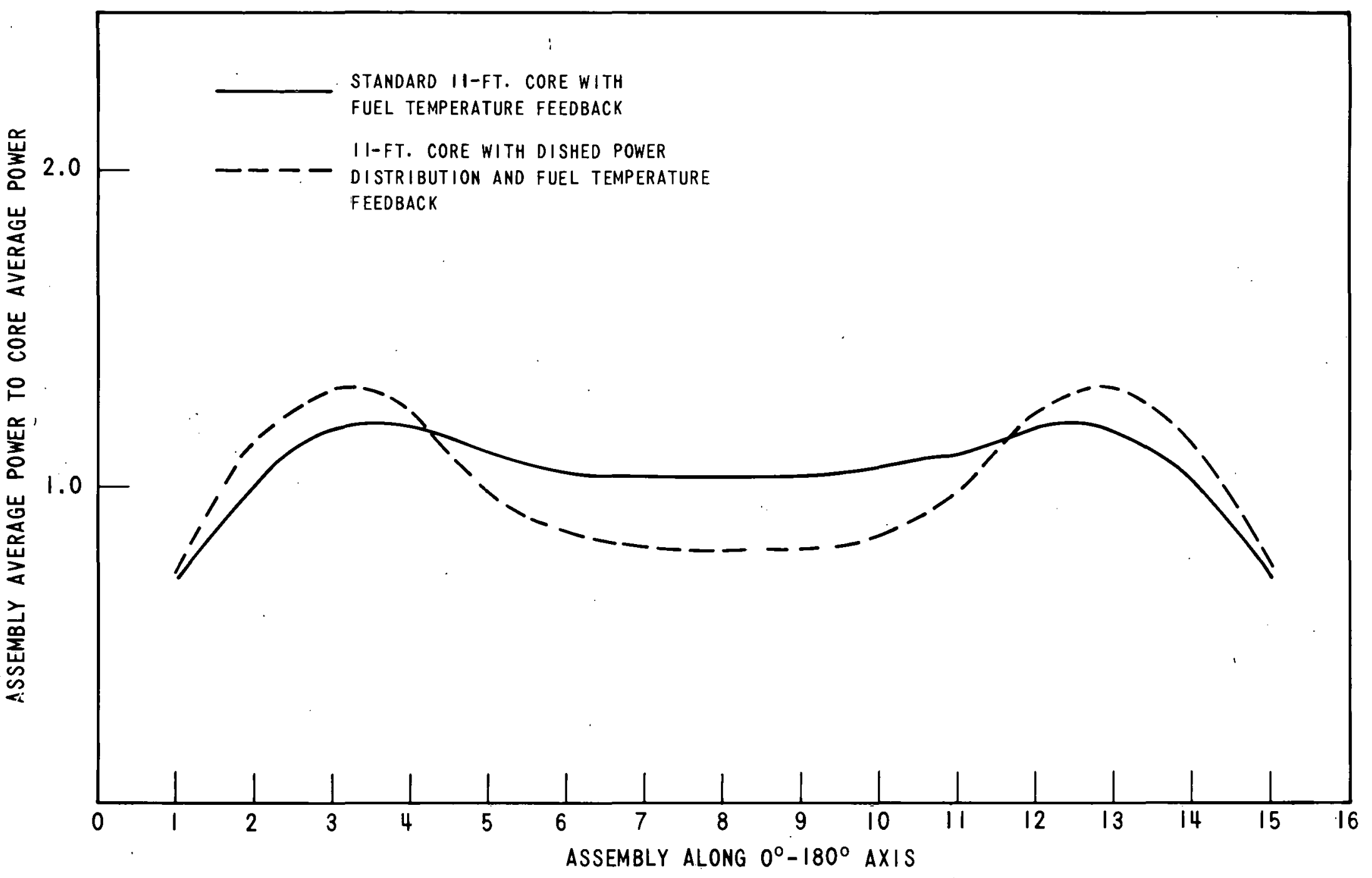

Figure 3-49. Steady-State Power Distribution for Dished and Normal 11-Foot Core with Fuel Temperature Feedback 


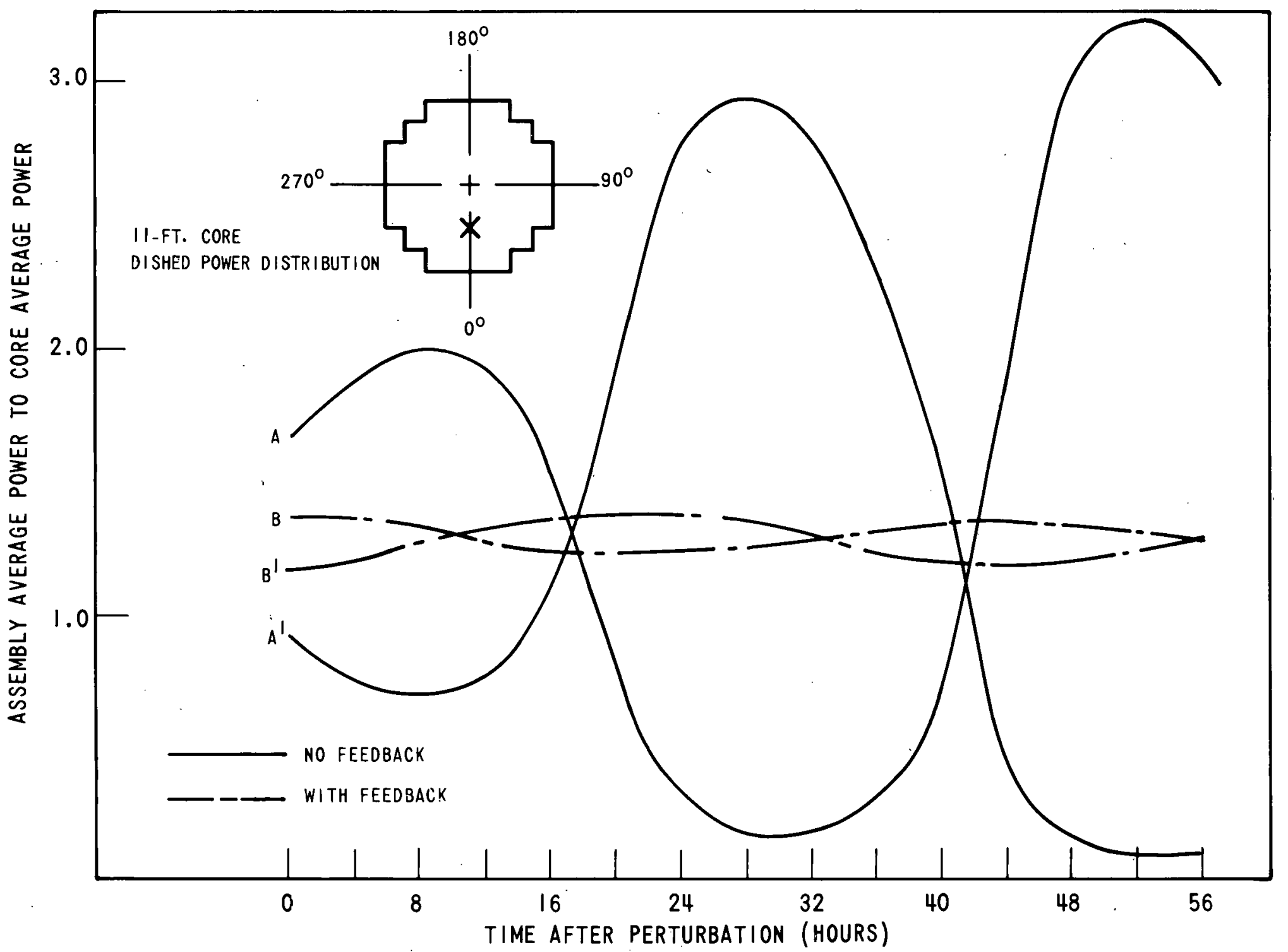

Figure 3-50. Xenon-Induced Oscillations with and without Fuel Temperature Feedback 


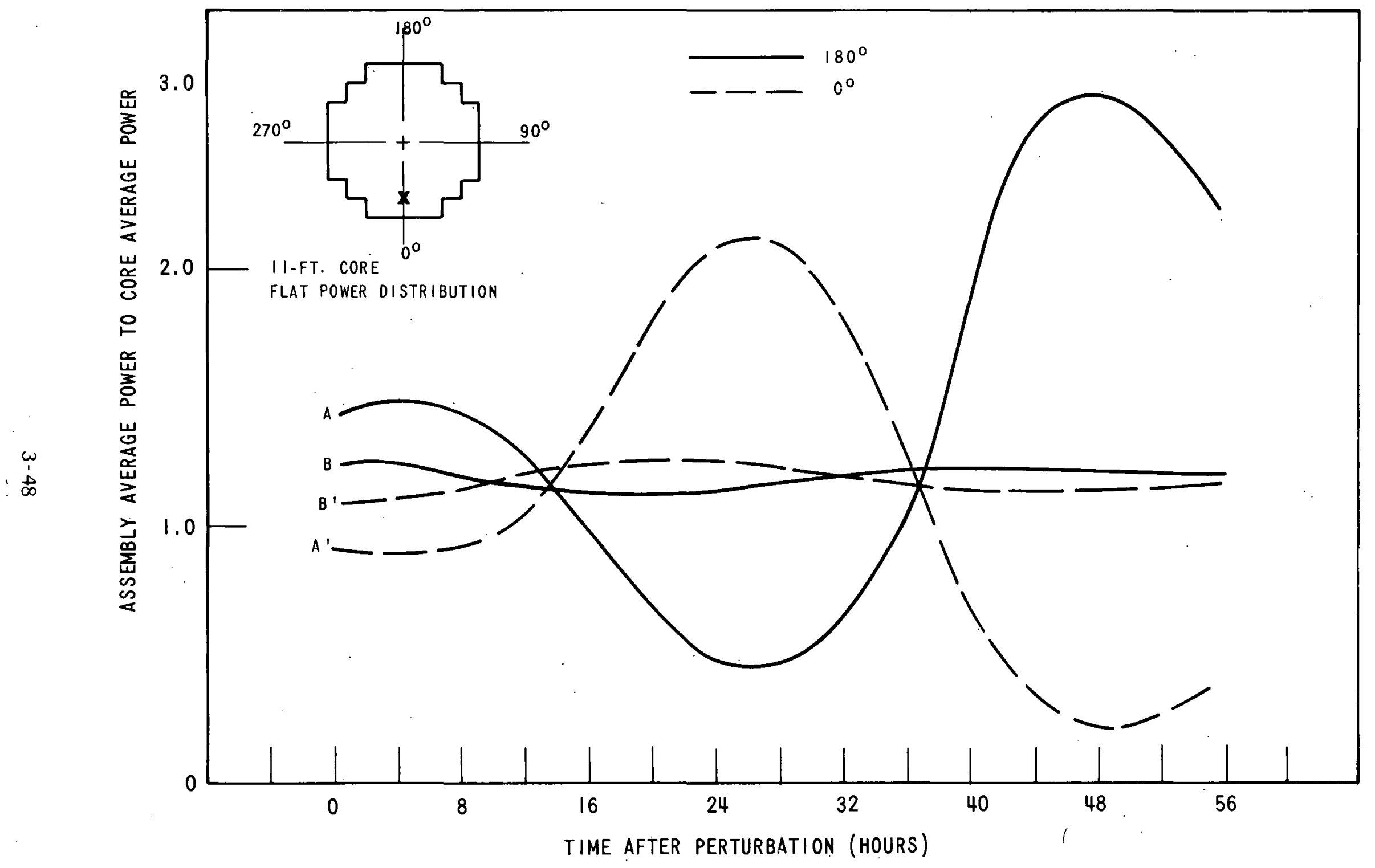

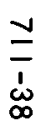

Figure 3-51. Xenon-Induced Oscillations with and without Doppler Feedback (Standard Core) 
flattened power distribution. This situation brings out a major difficulty in the use of multidimensional diffusion-theory codes for the analysis and control studies of xenon-induced oscillations. Although TURBO* is not representative of present-day capability in multidimensional calculations, it has been until now the only code which allowed the explicit representation of fuel temperature feedback in the $x-y$ plane. Running time with the temperature feedback included is, however, excessive -- being of the order of 15-30 minutes per time-step (on an IBM-7090). This factor precludes the use of shorter time-steps.

2. The cases studied here include only fuel temperature feedback, and thus correspond to a situation where the moderator temperature coefficient is zero. In the $x-y$ plane, the effects of fuel temperature and moderator density feedback are, to a first approximation, additive (as opposed to the axial plane, where the moderator density variations are dependent on an integral of the flux distribution). Therefore, any negative value of the moderator temperature coefficient will tend to stabilize the core against azimuthal or diametral oscillations. An estimate of the magnitude of this effect can be had from Figures 4-1 through 4-8 and equation (4.13).

The sensitivity of the stability characteristics to the fuel temperature feedback was studied with the one-dimensional program ZEST (Appendix D). The standard 11-foot core with a flattened power distribution was used as a basis for the calculations. Oscillations across a diameter in the $x-y$ plane were simulated in one-dimensional slab geometry. Two-hour time-steps were used in the calculations which, for the nominal fuel temperature feedback, result in slightly divergent oscillations.

ZEST includes a fairly complete treatment of fuel temperature feedback in that explicit correlations between fuel temperature and power density, fast absorption cross section and fuel temperature, and fast removal cross section 
and fuel temperature are used by the program. The sensitivity to the magnitude of fuel temperature feedback was studied by modifying the correlation between fuel temperature and power density. Calculations were performed where the slope $\partial \mathrm{T}_{f} / \partial \mathrm{p}$ was varied uniformly by \pm 20 percent from its nominal value. The resulting oscillations are shown in Figure 3-52, compared to the nominal case. Note that the effect of a -20 percent change in the magnitude of the power coefficient is relatively greater than +20 percent change in magnitude. This non-linear behavior is evident in the modal expansion results of Figure 4-13.

The space-dependent transfer function code SPARTA was employed in evaluating the effect of moderator density in core stability. Figure 3-53 shows the thermal gain response over the range of frequencies which are important for xenon feedback. These curves all include a nominal value for the Doppler coefficient. It is clear from the curves that to obtain an accurate picture of the magnitude of the oscillations, a moderator feedback treatment must be included in the analysis.

\subsection{EFFECT OF NON-LINEARITIES IN CORE STABILITY}

Certain nan-linear effects were noticed when xenon oscillations were allowed to grow in an uncontrolled manner. These effects are not of direct interest to the xenon study as they appear in a range outside that which would occur in a reactor. They are, however, of interest from the point of view of completeness of the study and could also be of interest in performance of a safety analysis.

The most important non-linear effect was found to be the saturation of the envelope surrounding the sinusoidal oscillations. This can be seen from Figure 3-54. Since the total core power remains constant by the suppression of the fundamental mode, it is obvious that the peak power on one side of the reactor is limited by the fact that the power cannot go below zero on the 




Figure 3-52. Effect of Variation of Fuel Temperature Feedback on Diametral Xenon-Induced Oscillations 


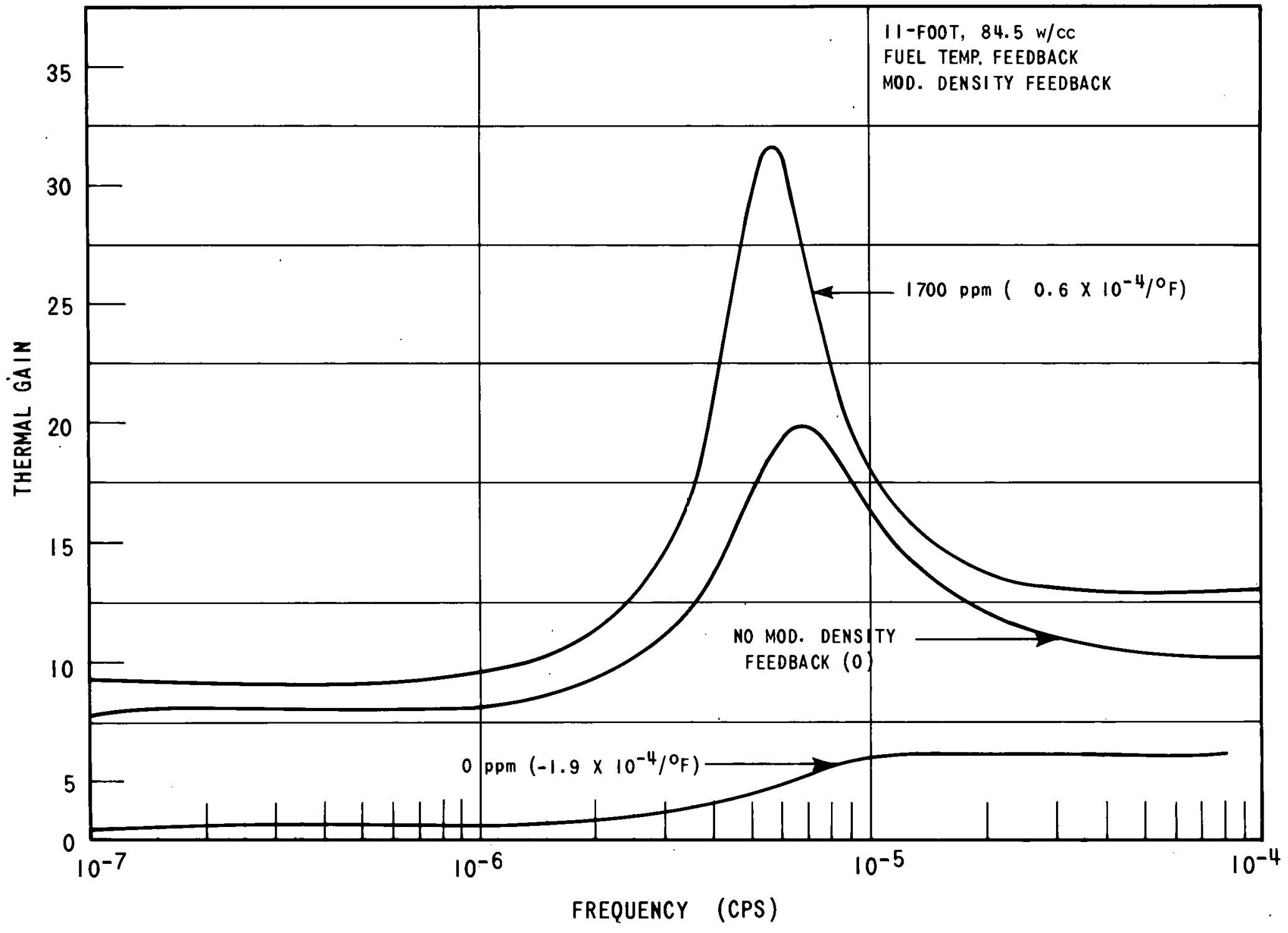

Figure 3-53. Effect of Moderator Density Feedback on Thermal Gain 


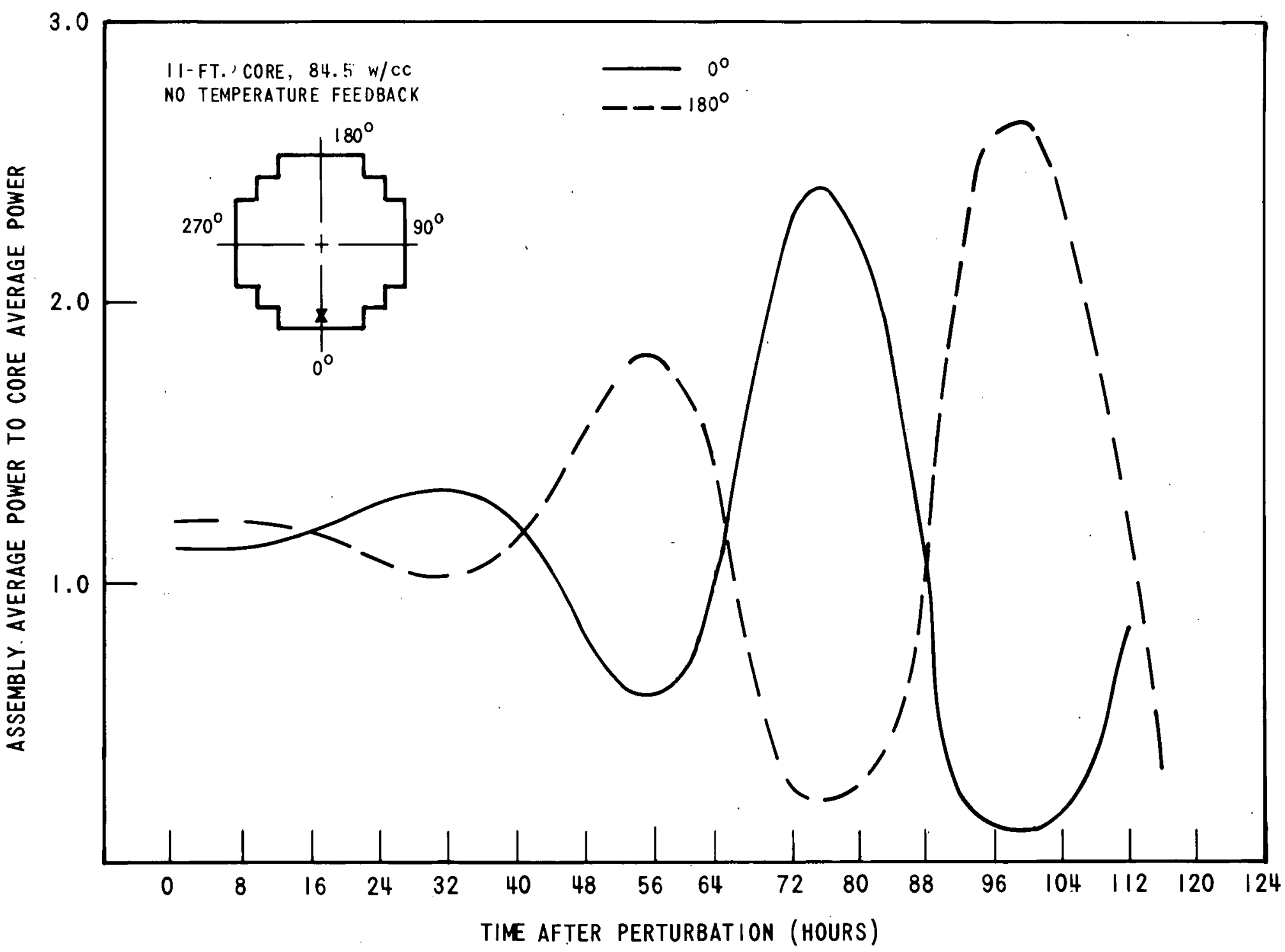

Figure 3-54. One-Dimensional Simulation of Azimuthal (Diametral.) Oscillations 
other. It is also of interest to note that for a period of some hours the total core power is produced almost solely from one side of the reactor. This is even more noticeable in the corresponding curve for the 15-foot core shown in Figure 3-55.

It was also found that the spatial flux distribution appeared to be a function of power level. This non-linear effect can be seen by examining Figures 3-56 and 3-57 which show the spatial power distributions for two 11-foot cores of average enrichment (2.6 percent) and flat equilibrium power distributions 24 hours after the perturbation has been removed. These distributions are for power levels of $2 / 3$ and $4 / 3$ the nominal value, respectively. It is observed that the higher the power level, the more the power contours become flattened into bands across the core. 


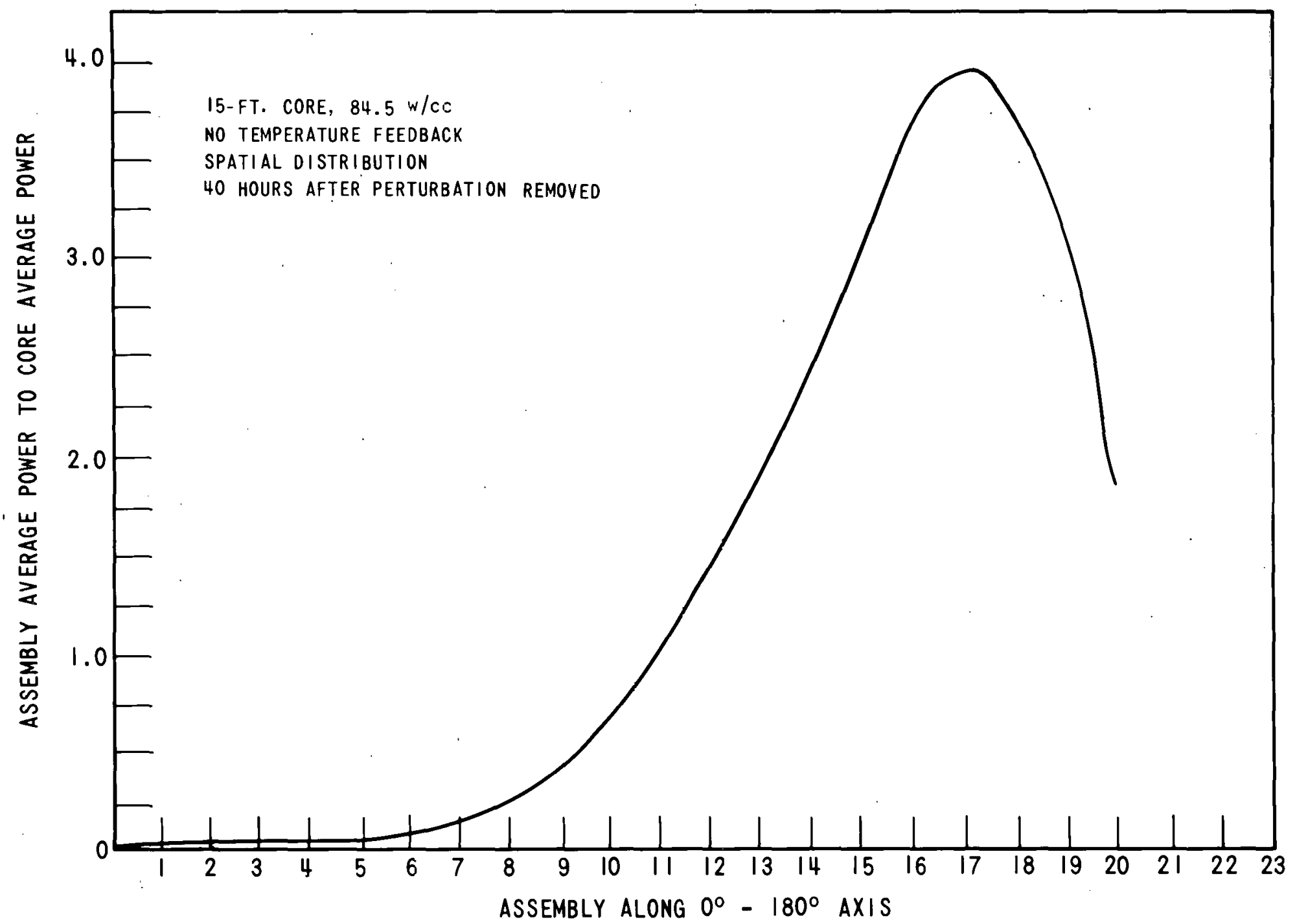

Figure 3-55. Spatial Power Distribution, 40 Hours after Removal of Perturbation for 15-Feot Core 


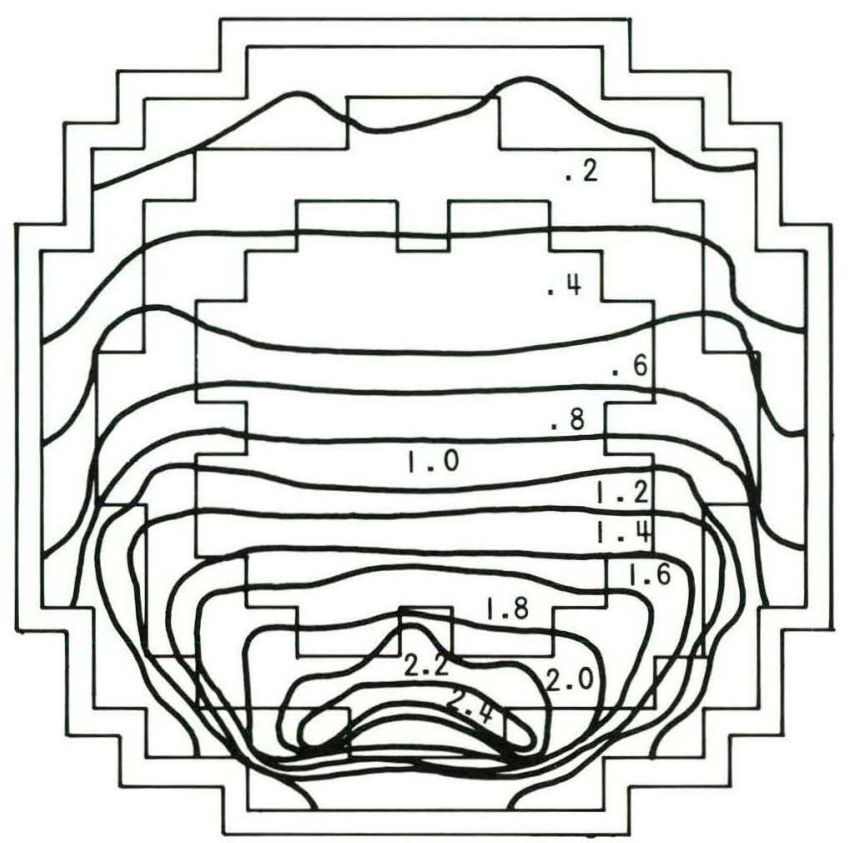

Figure 3-56. Contour Power Plot, 24 Hours after Perturbation for $2 / 3$ Power

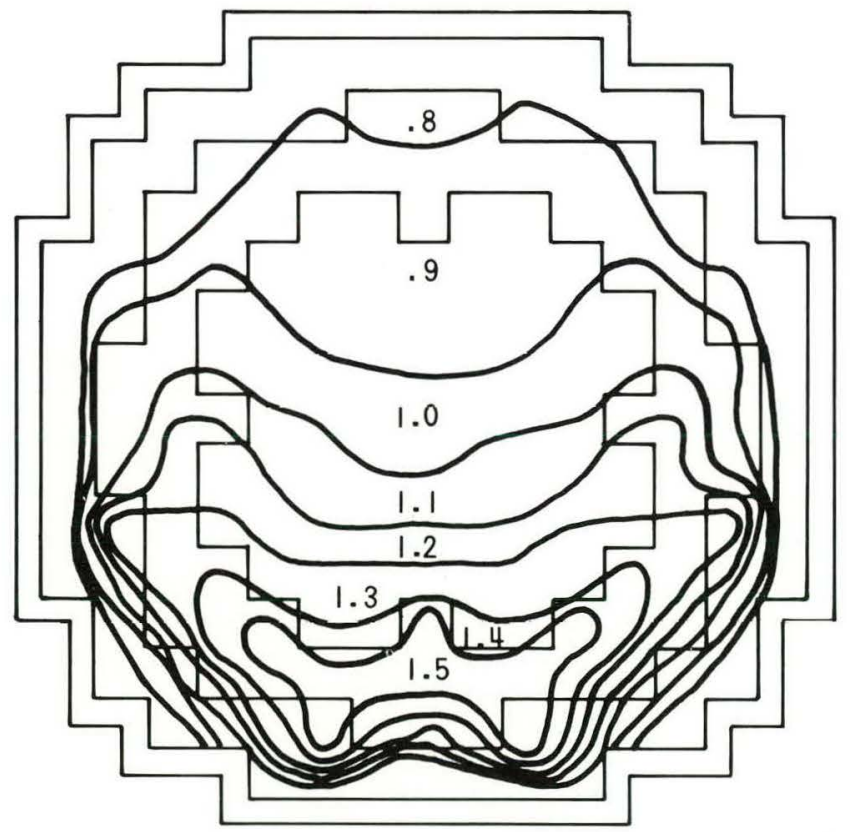

Figure 3-57. Contour Power Plot, 24 Hours after Perturbation for 4/3 Power 
SECTION 4

SENSITIVITY AND PARAMETRIC ANALYSES

Parametric analyses are useful in the evaluation of the stability characteristics of different core designs. Once a specific core design has been defined, such analyses are also essential in that the core parameters exhibit relatively large variations over the plant lifetime as a result of fuel burnup and control and fuel management actions. Thus, the spatial stability characteristics of a reactor may vary from cycle to cycle. It is necessary therefore, to design a detection and control system capable of coping with this variety of stability conditions.

In this section, the effect of changes in core parameters and physical data on spatial stability characteristics in large PWR's is evaluated. The analysis is on the effect on the stability index* and the oscillation period. The spatial characteristics in the two-dimensional plane were discussed in Section 3.

Section 4.1 presents a simplified modal theory to generate analytical functional relationships between the stability index and various core parameters. Comprehensive parametric curves relating stability index and oscillation period to core parameters are shown. These indicate the sensitivity of the core to the parameters in question, and provide a complete and continuous mapping of these variables through the ranges of interest. They may also be useful in evaluating numerical results. The limitations of modal theory were discussed in Section 2.4.

*The stability index used in this context is defined in Appendix $B$. 


\subsection{MODAL ANALYSES}

Equations (2.74), (2.75) and (2.76) have been used to calculate the stability index and the period for diametral oscillations in the $x-y$ plane as a function of various core parameters. Variations in core enrichment, core diameter, power density, power distribution, power coefficient, iodine yield, xenon yield and xenon cross section were studied. Simple functional relationships between the stability index and the various parameters listed above can be derived if the following simplifying approximations are made. The volumeaveraged value of $\bar{\phi}_{0}$ is used in lieu of $\bar{\phi}_{1}$ in equation (2.74). For a flat flux distribution this is exact, while for a sinusoidal distribution in an one-dimensional slab, $\bar{\phi}_{1} / \bar{\phi}_{0}=1.0667 .{ }^{[3]}$ We further assume

$$
\bar{\phi}_{0}=\frac{p}{\Sigma_{f} k}
$$

where $p$ is the average core power density, $k$ is a conversion factor converting fissions/sec to watts, and $\Sigma_{f}$ is an effective fission cross section for the core-averaged fuel enrichment:

$$
\Sigma_{f}=\Sigma_{f}+\Sigma_{t h} \frac{\phi_{f}}{\phi_{t h}}
$$

where subscripts th and $f$ refer to the thermal and fast groups, respectively, in a two-group scheme. The average equilibrium xenon concentration (relative to the saturation value) is calculated from

$$
\bar{x}_{0}=\frac{\bar{\phi}_{0} \sigma_{x}}{\lambda_{x}+\bar{\phi}_{0} \sigma_{x}}
$$

The error introduced by the use of (4.3) has been shown by Randall and St. John ${ }^{[3]}$ to be small. In reference (3), a flux distribution index, $\Lambda_{1}$, is introduced, such that the eigenvalue $\mu_{1}^{2}$ is defined as

$$
\mu_{1}^{2}=\Lambda_{1} B_{0}^{2}
$$


where $B_{0}^{2}$ is the fundamental geometric buckling. Here a reduced index $\Gamma$ is introduced, which is defined as

$$
\Gamma=\frac{\mathrm{B}_{1}^{2}-\mathrm{B}_{\mathrm{O}}^{2}}{\Lambda_{1} \mathrm{~B}_{\mathrm{O}}^{2}}
$$

where $B_{1}^{2}$ is the eigenvalue of the first overtone of the geometric modes. If $L$ is a characteristic extrapolated dimension (core height or diameter), $\mu_{1}^{2}$ can be written as

$$
\mu_{1}^{2}=\frac{\xi}{\Gamma L}
$$

where $\xi=0.031871$ for axial oscillations, $\xi=0.038319$ for azimuthal oscillations, and $L$ is in units of feet. For a slab, $\Gamma$ is related to $\Lambda_{1}$ by

$$
\Gamma=\frac{3}{\Lambda_{1}}
$$

For a sinusoidal distribution, $\Lambda_{1}=3(\Gamma=1)$, and for a flat distribution, $\Lambda_{1}=1(\Gamma=3)$. If the flux is flattened in all directions, the values of $\Gamma$ for diametral oscillations can be approximated by those for axial oscillations.

The coefficient $a_{x}$ is calculated from

$$
a_{x}=\frac{\rho x_{,} \infty}{M^{2}}
$$

where $\rho_{x, \infty}$ is the reactivity effect of saturated xenon and $M^{2}$ is the migration area. To a good approximation,

$$
\rho_{\mathrm{x}, \infty}=-\frac{\mathrm{k}_{\mathrm{th}}}{\sum_{\mathrm{a}_{\mathrm{th}}}} \sum_{\mathrm{f}} \mathrm{y}_{\mathrm{T}}=-\mathrm{c} \sum_{\mathrm{f}} \mathrm{y}_{\mathrm{T}}
$$

where $k_{t h}$ is the contribution of the thermal group to $k_{\text {eff }}$ of the core, and $\Sigma_{f}$ is defined in equation (4.2). 
The coefficient $a_{T}$ can be written in terms of a net power coefficient $(\partial \rho / \partial P):$

$$
a_{T}=\frac{(\partial \rho / \partial P) V \Sigma_{f} \kappa\left(10^{-6}\right)}{M^{2}}
$$

where $\mathrm{V}$ is the core volume in $\mathrm{cm}^{3}$ and $(\partial \rho / \partial P)$ is in units of reactivity change per MWT. Note that with fuel temperature and moderator density feedback in the $x-y$ plane, $\partial \rho / \partial P$ is defined as

$$
\partial \rho / \partial P=\frac{\partial \rho}{\partial T_{f}} \frac{\partial T_{f}}{\partial P}+\frac{\partial \rho}{\partial T_{m}} \frac{\partial T_{m}}{\partial P}
$$

where $T_{f}$ and $T_{m}$ are the fuel and moderator temperatures, respectively. When the average core enrichment is varied, we introduce the following approximation

$$
\Sigma_{f}=\Sigma_{f}^{*} \frac{\varepsilon}{\varepsilon}
$$

where $\varepsilon$ is the U-235 enrichment, and superscript * refers to a nominal value. We also assume that $\rho_{x, \infty}$ is independent of emrichment.

With the above approximations, the dependence of the stability index $b$ on the various core parameters, each taken individually, can be represented generally as

$$
b=\frac{a_{0} x^{3}+a_{1} x^{2}+a_{2} x+a_{3}}{a_{4} x^{2}+a_{5} x+a_{6}}
$$

where $x$ represents the core parameter of interest. The constants $a_{i}$ in equation (4.13) are defined as follows:

1. $\mathrm{x}=\mathrm{U}-235$ enrichment, $\varepsilon$ :

$$
a_{1}=\left(\lambda_{1}+\lambda_{x}\right) \lambda_{x}
$$




$$
\begin{aligned}
& a_{2}=\left(\lambda_{i}+2 \lambda_{x}\right) A-D \\
& a_{3}=A^{2}+C \\
& a_{4}=\lambda_{x} \\
& \mathrm{a}_{5}=\mathrm{A} \\
& a_{0}=a_{6}=0 \\
& A=\frac{\sigma_{x} p^{*}}{\sum_{f}^{N} \kappa} \\
& D=B \gamma_{i}{ }^{\lambda} x \\
& C=B A\left(1-\gamma_{x}\right) \\
& B=-\frac{c \sigma_{x} p t^{*} y_{T}}{k M^{2}\left[\mu_{1}^{2}-(\partial \rho / \partial P) V \rho\left(10^{-6}\right)\right]}
\end{aligned}
$$

2. $x=$ core size, L:

$$
\begin{aligned}
& a_{1}=\frac{A D \Gamma}{\xi}-C \\
& a_{3}=A \\
& a_{4}=\frac{D \Gamma}{\xi} \\
& a_{6}=1 \\
& a_{0}=a_{2}=a_{5}=0 \\
& A=\lambda_{i}+\lambda_{x}+\sigma_{x} \bar{\phi}
\end{aligned}
$$

4-5 
4. $x=$ power distribution, $\Gamma$ :

$$
\begin{aligned}
& a_{2}=\frac{A D L^{2}}{\xi}-C \\
& \mathrm{a}_{3}=\mathrm{A} \\
& a_{5}=\frac{D L^{2}}{\xi} \\
& a_{6}=1 \\
& a_{0}=a_{1}=a_{4}=0 \\
& A=\lambda_{i}+\lambda_{x}+\sigma_{x} \bar{\phi} \\
& D=-\frac{(\partial \rho / \partial P) V \rho\left(10^{-6}\right)}{M^{2}} \\
& C=\frac{c \sigma_{x} y_{T} \rho L^{2}}{\kappa M^{2} \xi}\left[\frac{\bar{\phi} \sigma_{x}}{\lambda_{x}+\bar{\phi} \sigma_{x}}-\gamma_{x}\right]
\end{aligned}
$$

5. $x=$ power coefficient, $\partial \rho / \partial P$ :

$$
\begin{aligned}
& a_{2}=-A C \\
& a_{3}=A \mu_{1}^{2}-B \\
& a_{5}=-C \\
& a_{6}=\mu_{1}^{2} \\
& a_{0}=a_{1}=a_{4}=0 \\
& A=\lambda_{i}+\lambda_{x}+\sigma_{x} \bar{\phi}
\end{aligned}
$$




$$
\begin{aligned}
& D=-\frac{(\partial \rho / \partial P) V \rho\left(10^{-6}\right)}{M^{2}} \\
& C=\frac{c \sigma_{x} y_{T} \rho \Gamma}{k M^{2} \xi}\left[\frac{\bar{\phi} \sigma_{x}}{\lambda_{x}+\bar{\phi} \sigma_{x}}-\gamma_{x}\right]
\end{aligned}
$$

3. $x=$ flux level, $\bar{\phi}$ :

$$
\begin{aligned}
& a_{0}=-E \sigma_{x}^{2} \\
& a_{1}=-\left(\lambda_{i}+\lambda_{x}\right) E \sigma_{x}+\sigma_{x}\left(\mu_{1}^{2} \sigma_{x}-E \lambda_{x}\right)+B \sigma_{x}\left(1-\gamma_{x}\right) \\
& a_{2}=\left(\lambda_{i}+\lambda_{x}\right)\left(\mu_{1}^{2} \sigma_{x}-E \lambda_{x}\right)+\mu_{1}^{2} \lambda_{x} \sigma_{x}-B \gamma_{x} \lambda_{x} \\
& a_{3}=\left(\lambda_{i}+\lambda_{x}\right) \tilde{\lambda}_{x} \mu_{1}^{2} \\
& a_{4}=-E \sigma_{x} \\
& a_{5}=\mu_{1}^{2} \sigma_{x}-E \lambda_{x} \\
& a_{6}=\mu_{1}^{2} \lambda_{x} \\
& E=\frac{(\partial \rho / \partial P) V \Sigma_{f^{k}}\left(10^{-6}\right)}{M^{2}} \\
& B=-\frac{c \sigma_{x} \Sigma_{f} y_{T}}{M^{2}}
\end{aligned}
$$

Note that equation (4.1) can be used with the above to find the dependence on power density. 


$$
\begin{aligned}
& C=\frac{V \rho\left(10^{-6}\right)}{M^{2}} \\
& B=\frac{c \sigma_{x} y_{T} \rho}{k M^{2}}\left[\frac{\bar{\phi} \sigma_{x}}{\lambda x+\bar{\phi} \sigma_{x}}-\gamma_{x}\right] .
\end{aligned}
$$

6. $x=$ total xenon yield, $y_{T}:\left(\gamma_{x}\right.$ constant $)$

$$
\begin{aligned}
& a_{2}=-B \\
& a_{3}=A \\
& a_{6}=1 \\
& a_{0}=a_{1}=a_{4}=a_{5}=0 \\
& A=\lambda_{i}+\lambda_{x}+\sigma_{x} \bar{\phi} \\
& B=\frac{c \sigma_{x} \rho}{k M^{2}}\left[\frac{\bar{\phi} \sigma_{x}}{\mu_{1}^{2}-\frac{(\partial \rho / \partial P)}{\phi} \bar{\phi}_{x}\left(10^{-6}\right)}\right.
\end{aligned}
$$

7. $x=$ xenon cross section, $\sigma_{x}$ :

$$
\begin{aligned}
& a_{1}=\bar{\phi}\left[\bar{\phi}+B\left(1-\gamma_{x}\right)\right] \\
& a_{2}=\bar{\phi}\left(A-\lambda_{x}\right)-B \gamma_{x} \lambda_{x} \\
& a_{3}=A \lambda_{x} \\
& a_{5}=\bar{\phi}
\end{aligned}
$$

$4-8$ 


$$
\begin{aligned}
& a_{6}=\lambda_{x} \\
& a_{0}=a_{4}=0 \\
& A=\lambda_{i}+\lambda_{x} \\
& B=\frac{c y_{T} \rho}{M^{2}\left[\mu_{1}^{2}-\frac{(\partial \rho / \partial P) V_{0}\left(10^{-6}\right)}{M^{2}}\right]}
\end{aligned}
$$

8. $x=$ fractional xenon yield, $\gamma_{x}:\left(y_{T}\right.$ constant $)$

$$
\begin{aligned}
a_{2} & =B \\
a_{3} & =A-B C \\
a_{6} & =1 \\
a_{0} & =a_{1}=a_{4}=a_{5}=0 \\
A & =\lambda_{1}+\lambda_{x}+\sigma_{x} \bar{\phi} \\
B & =\frac{c \sigma_{x} y_{T} \rho}{k M^{2}\left[\mu_{1}^{2}-\frac{(\partial \rho / \partial P) V \rho\left(1 \cdot 0^{-6}\right)}{M^{2}}\right]} \\
C & =\frac{\lambda_{x}+\bar{\phi}_{x} \sigma_{x}}{.}
\end{aligned}
$$

Figures 4-1 through 4-8 show plots of the stability index for diametral oscillations as a function of core parameters. The standard parameters used as a basis for the calculations are those of the 11-foot diameter core (12-foot axial length) with an average $U-235$ enrichment of 2.6 a/o, average power density $84.5 \mathrm{w} / \mathrm{cc}$ and a flat flux distribution. Other parameters 


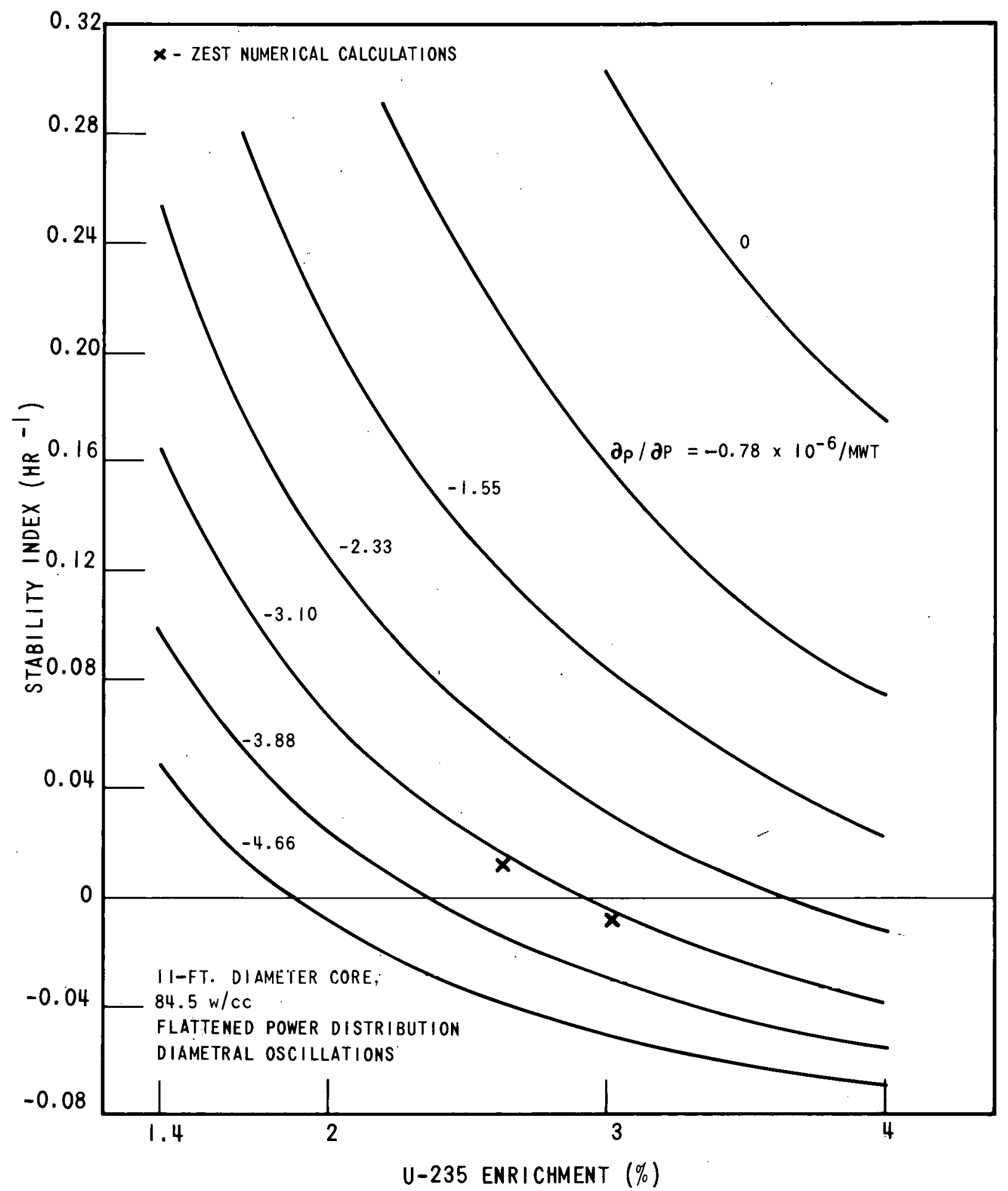

Figure 4-1. Variation of Stability Index with U-235 Enrichnent - Modal Analysis 


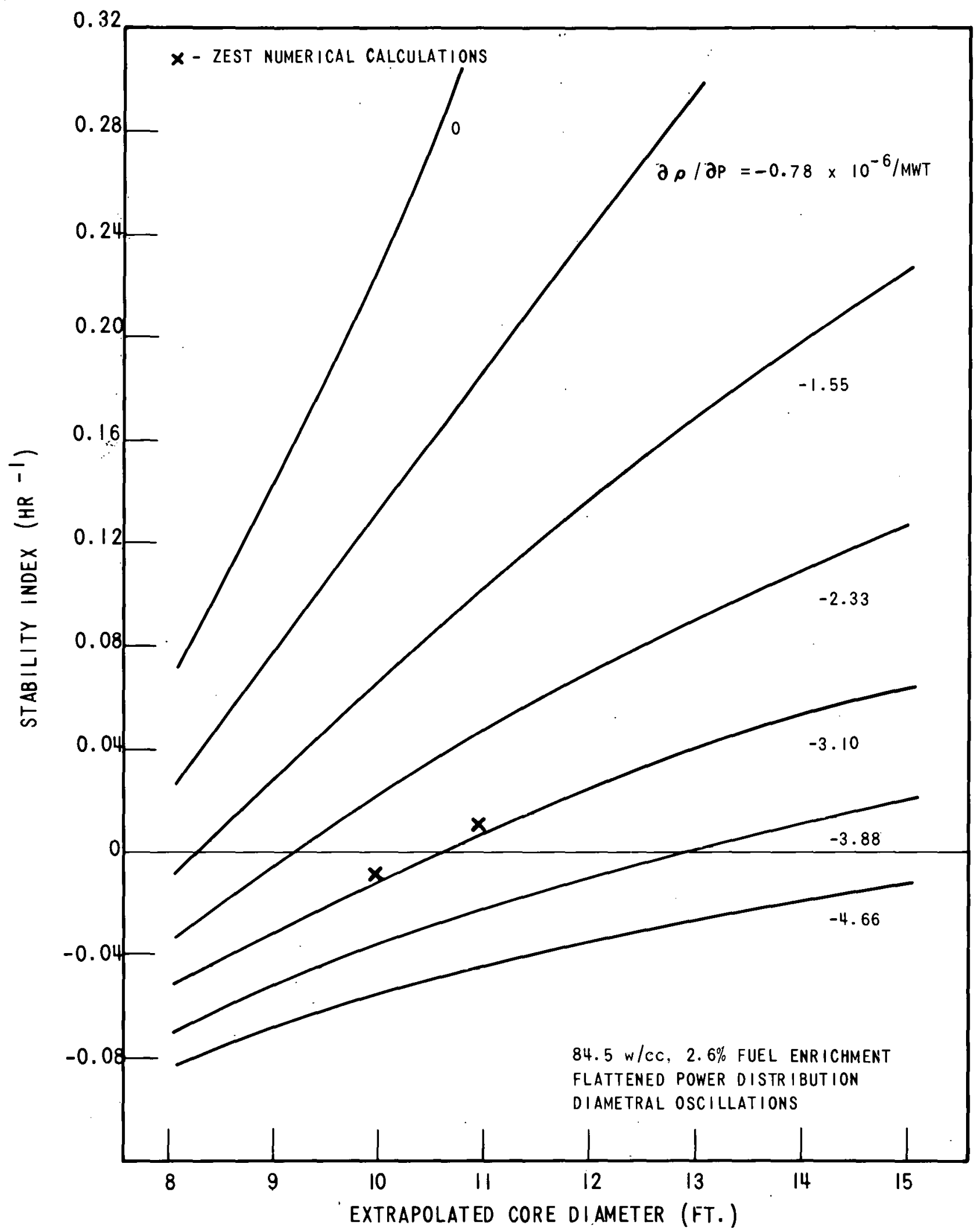

Figure 4-2. Variation of Stability with Core Diameter - Modal Analysis 


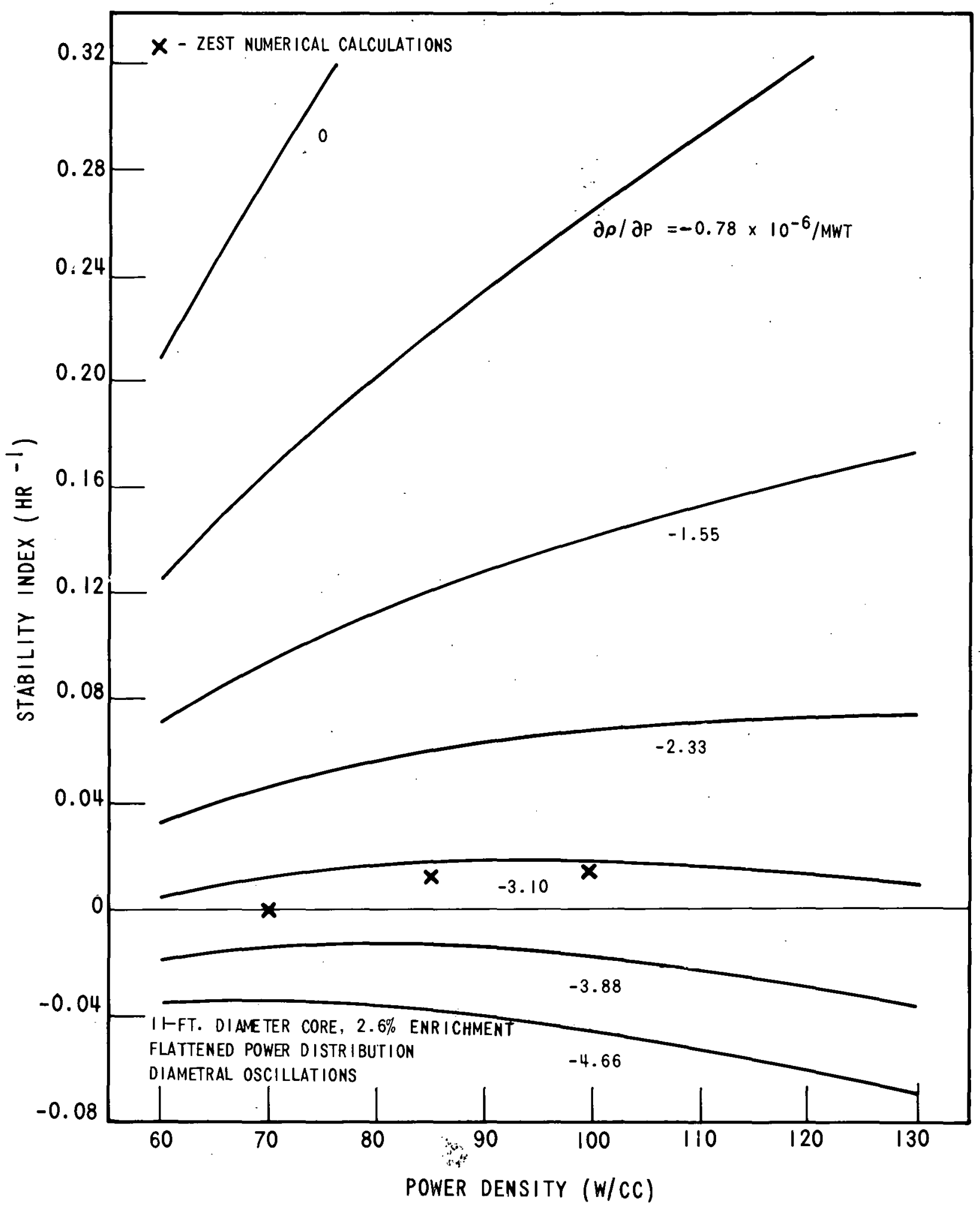

Figure 4-3. Variation of Stability Index with Average Fower Density - Modal Analys is 


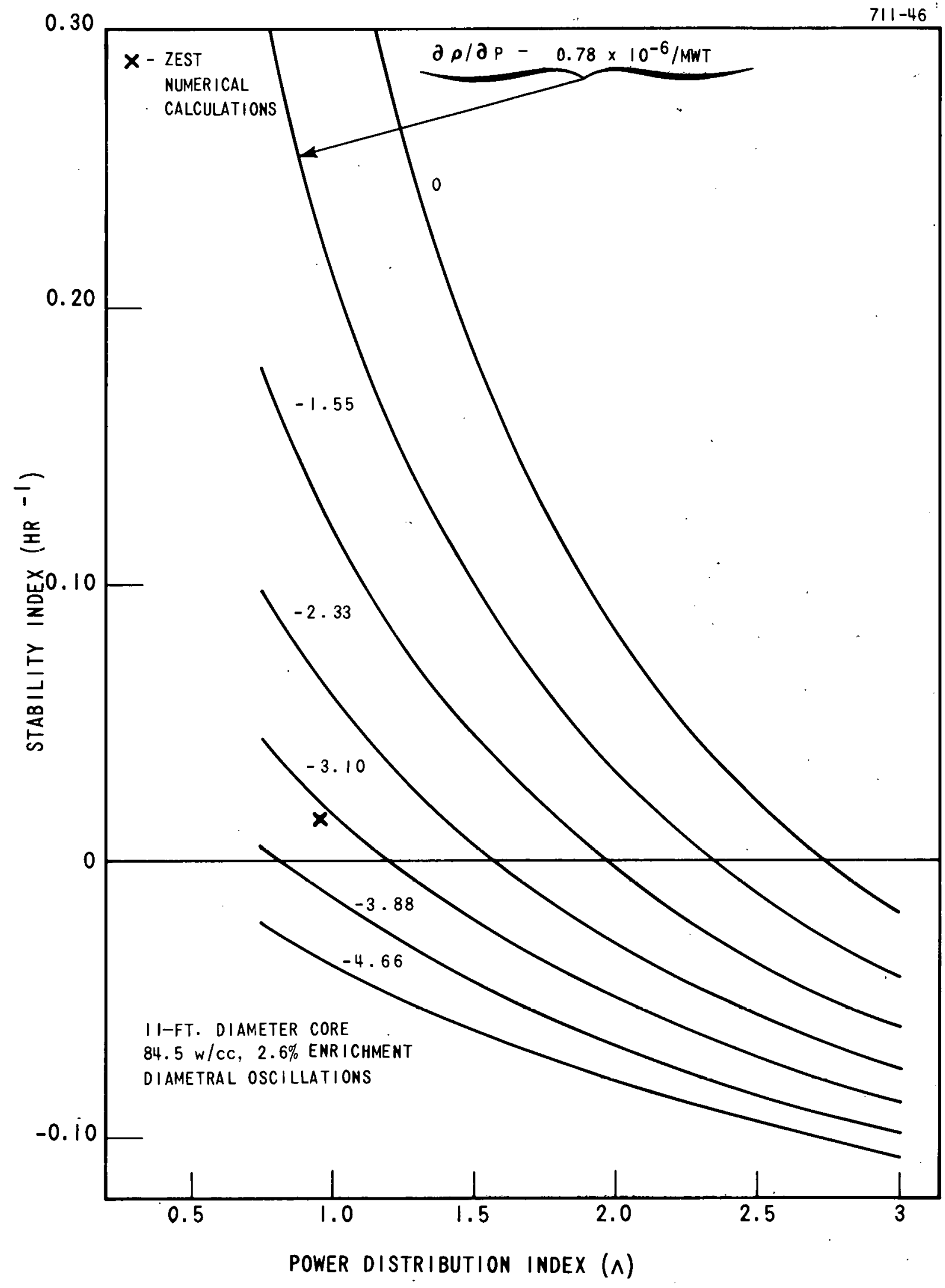

Figure 4-4. Variation of Stability Index with Power Distribution - Modal Analysis 


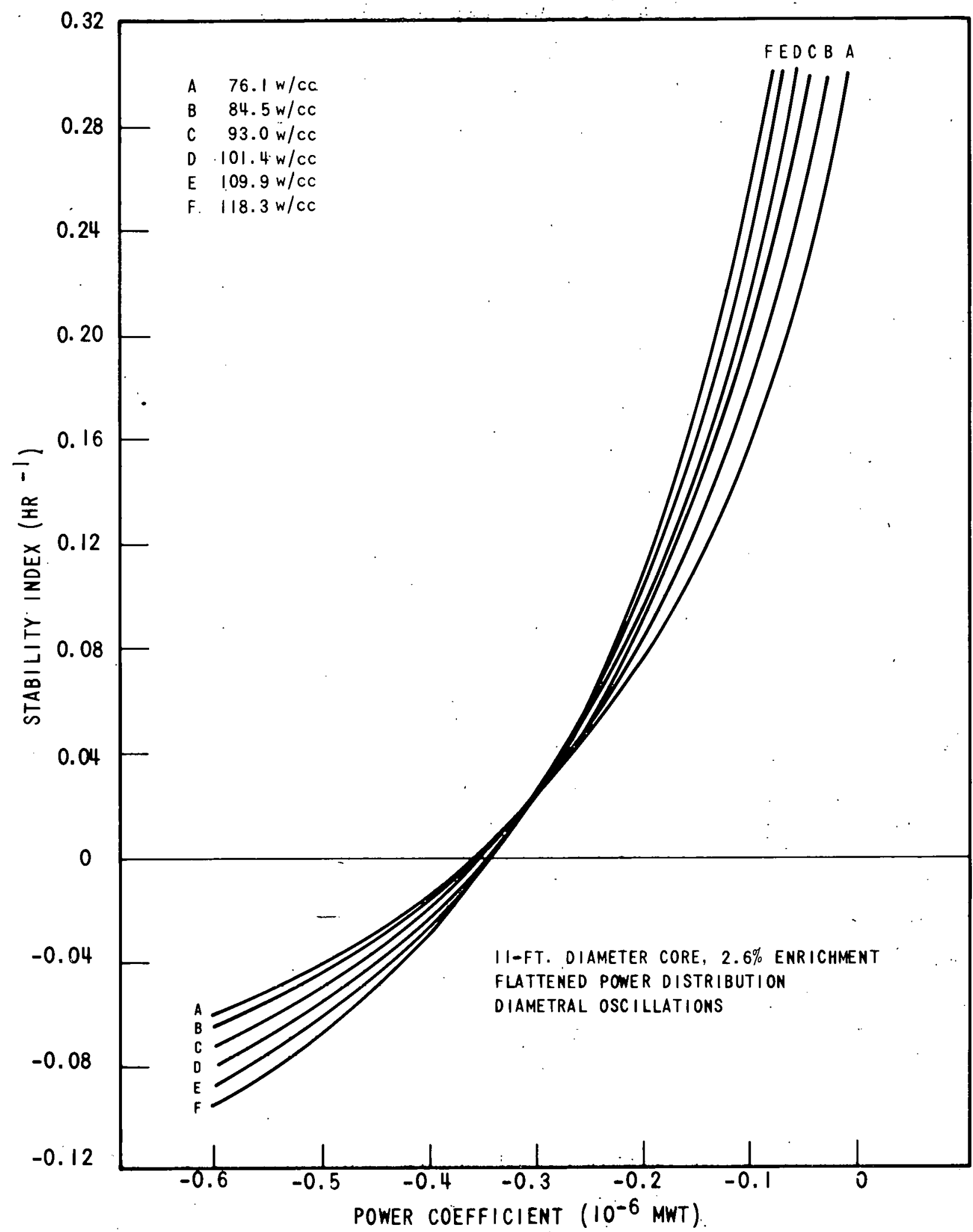

Figure 4-5. Variation of Stability Index with Net Power Coefficient - Modal Analysis 


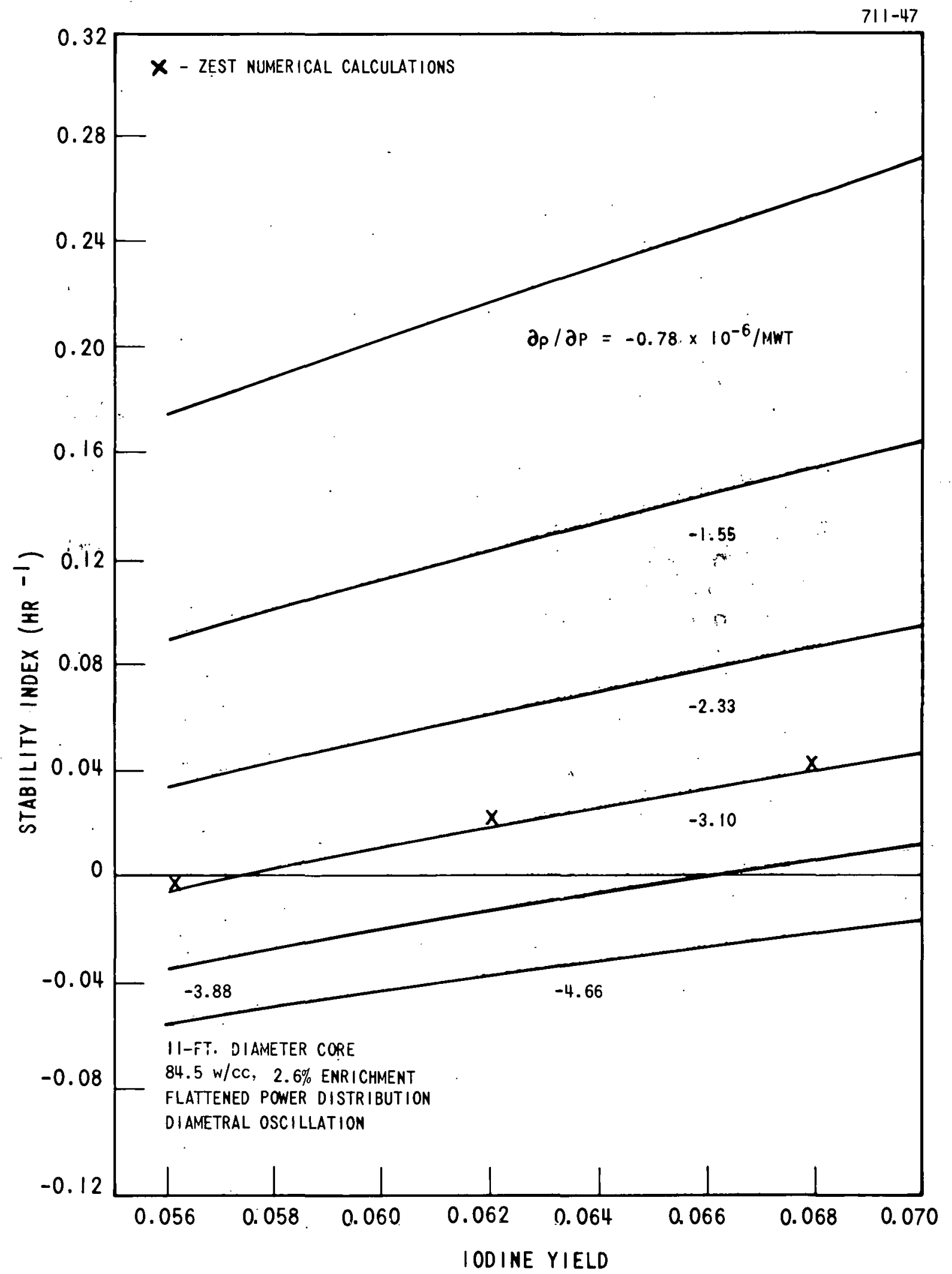

Figure 4-6. Variation of Stability Index with I-135 Yield - Modal Analysis 


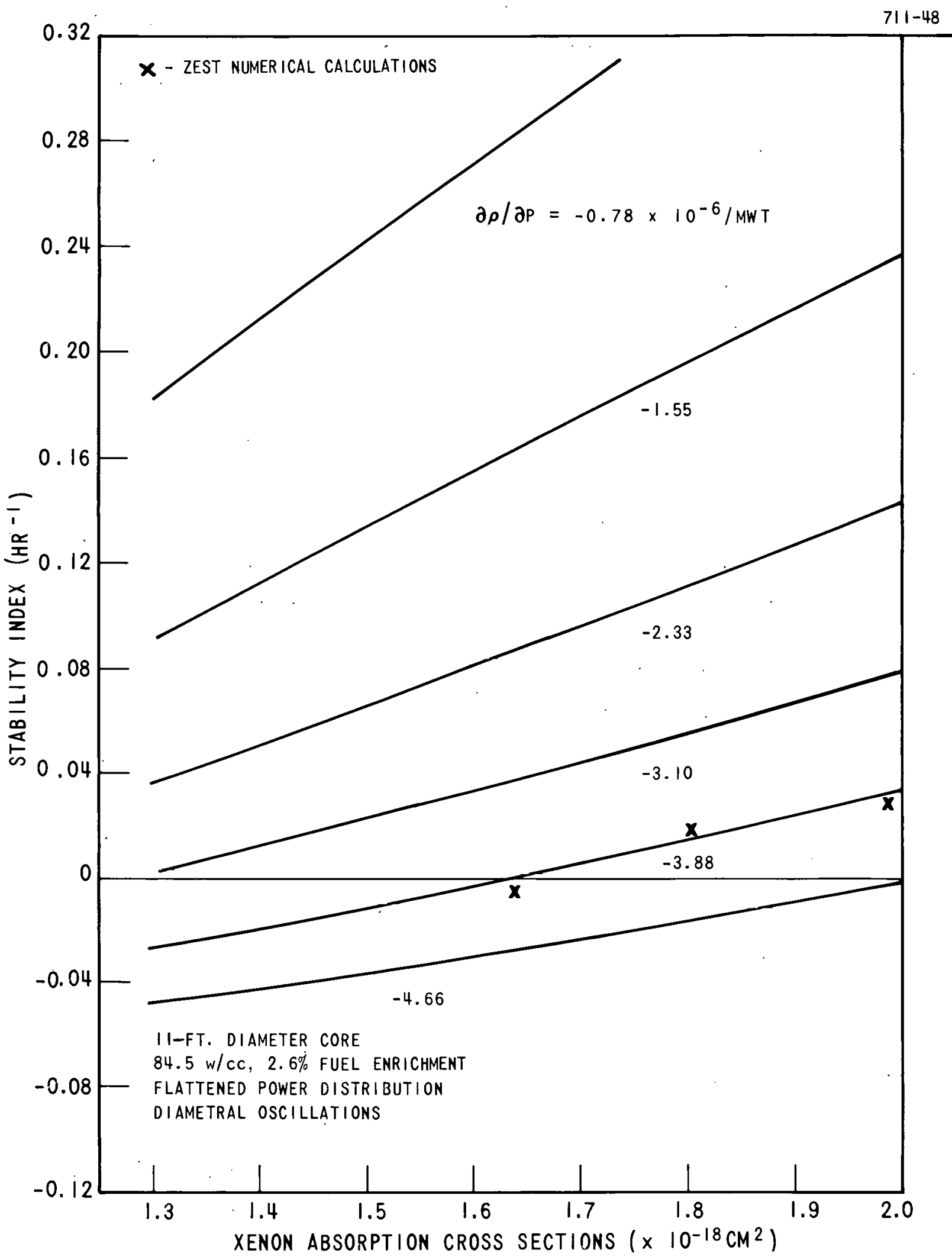

Figure 4-7. Variation of Stability Index with Xenon Absorption Cross Section-Modal Analysis 


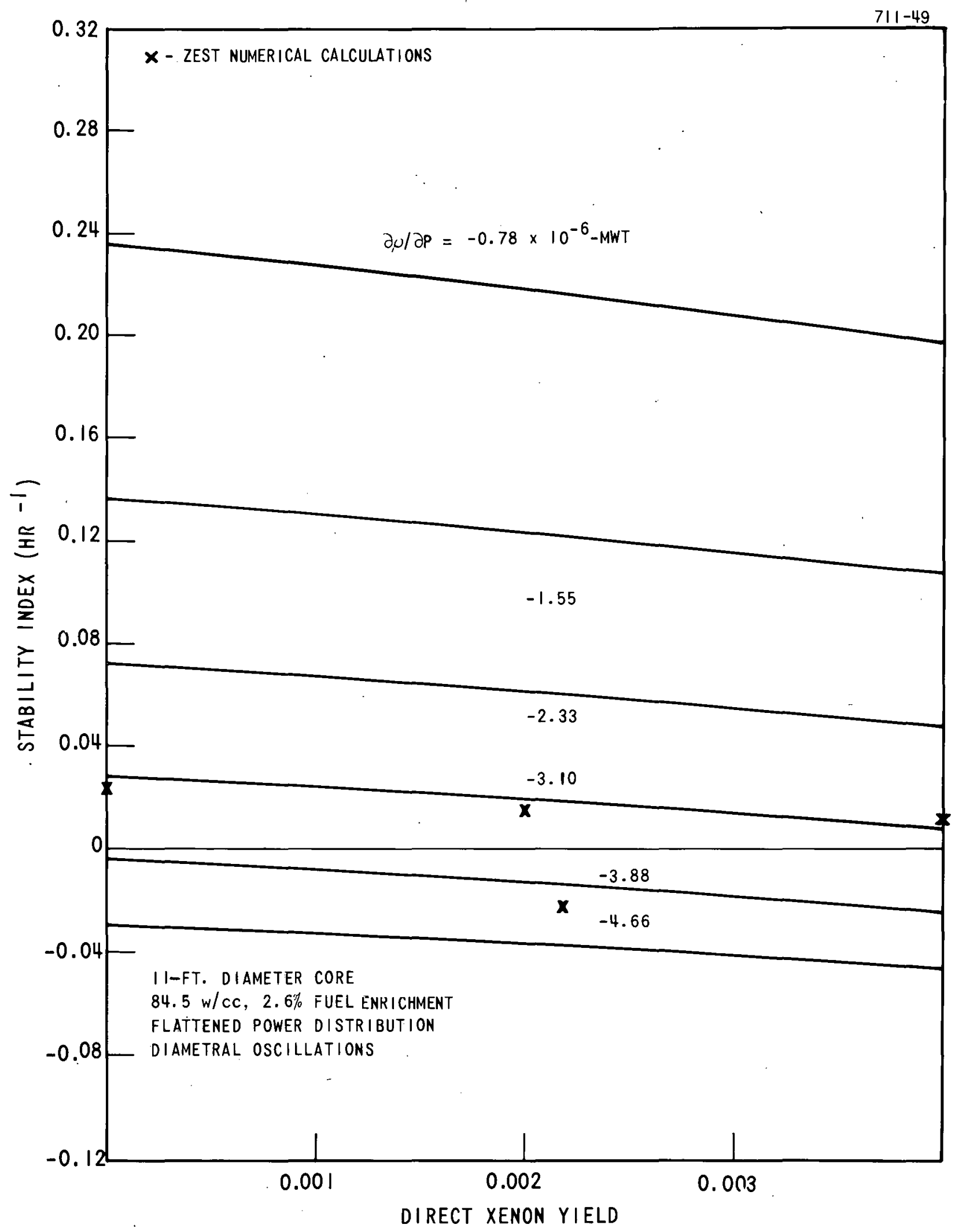

Figure 4-8. Variation of Stability Index with Direct Xe-135 Yield. Modal Analysis 
used in the calculations are:

$$
\begin{aligned}
\lambda_{i} & =2.88 \times 10^{-5} \mathrm{sec}^{-1} \\
\lambda_{x} & =2.11 \times 10^{-5} \mathrm{sec}^{-1} \\
\sigma_{x} & =1.622 \times 10^{-18} \mathrm{~cm}^{2} \\
y_{i} & =0.062 \\
y_{x} & =0.002
\end{aligned}
$$

The nominal power coefficient due to fuel temperature change is $-3.10 \times 10^{-6} / \mathrm{MWT}$. The total power coefficient for different values of moderator temperature coefficient can be obtained from equation (4.11). For example, taking $\partial \mathrm{T}_{\mathrm{m}} / \partial \mathrm{p}=0.3^{\circ} \mathrm{F} / \mathrm{w} / \mathrm{cc}, \partial \rho / \partial \mathrm{p}$ takes on values of $-4.5 \times 10^{-6} / \mathrm{MWt}$ and $-1.7 \times 10^{-6} / \mathrm{MWt}$ for moderator temperature coefficients of $-1.5 \times 10^{-4} /{ }^{\circ} \mathrm{F}$ and $+1.5 \times 10^{-4} /{ }^{\circ} \mathrm{F}$, respectively.

A number of general observations can be made from Figures 4-1 through 4-8. It is noted that the more negative the power coefficient, the less sensitive. is the stability index to variations in core parameters. It is also observed that sensitivity to core parameters, and in particular to the temperature feedback, is increased as the flux distribution is flattened. The effect of a change in power coefficient on the stability index is seen to be relatively decreased as the power coefficient becomes more negative. It is also observed that the production of plutonium, with a relatively larger value of I-135 yield than U-235, is a destabilizing factor.

Similar curves for the variation of the oscillation period with core parameters are given in Figures 4-9 through 4-16. The stability thresholds obtained from Figures 4-1 through 4-8 are shown in the figures. It is observed that the critical period of the stability threshold is independent of the extrapolated core size and the steady-state power distribution. This behavior has been noted by Randall and St. John [3] and by Lellouche. [8] The critical period, however, appears to be a function of enrichment, power level, power coefficient and Xe-135 absorption cross section. 


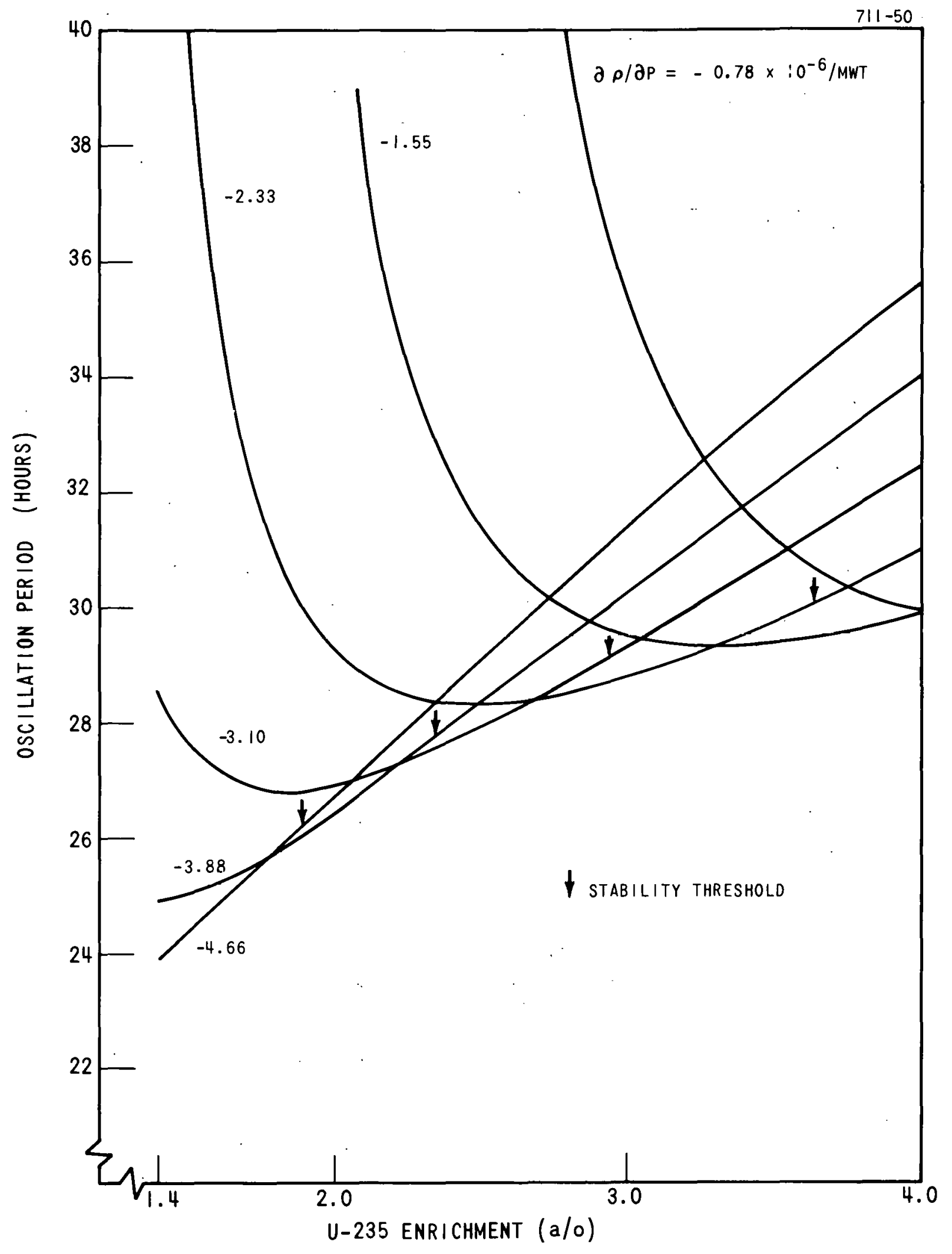

Figure 4-9. Variation of Oscillation Period with U-235 Enrichment - Modal Analysis 


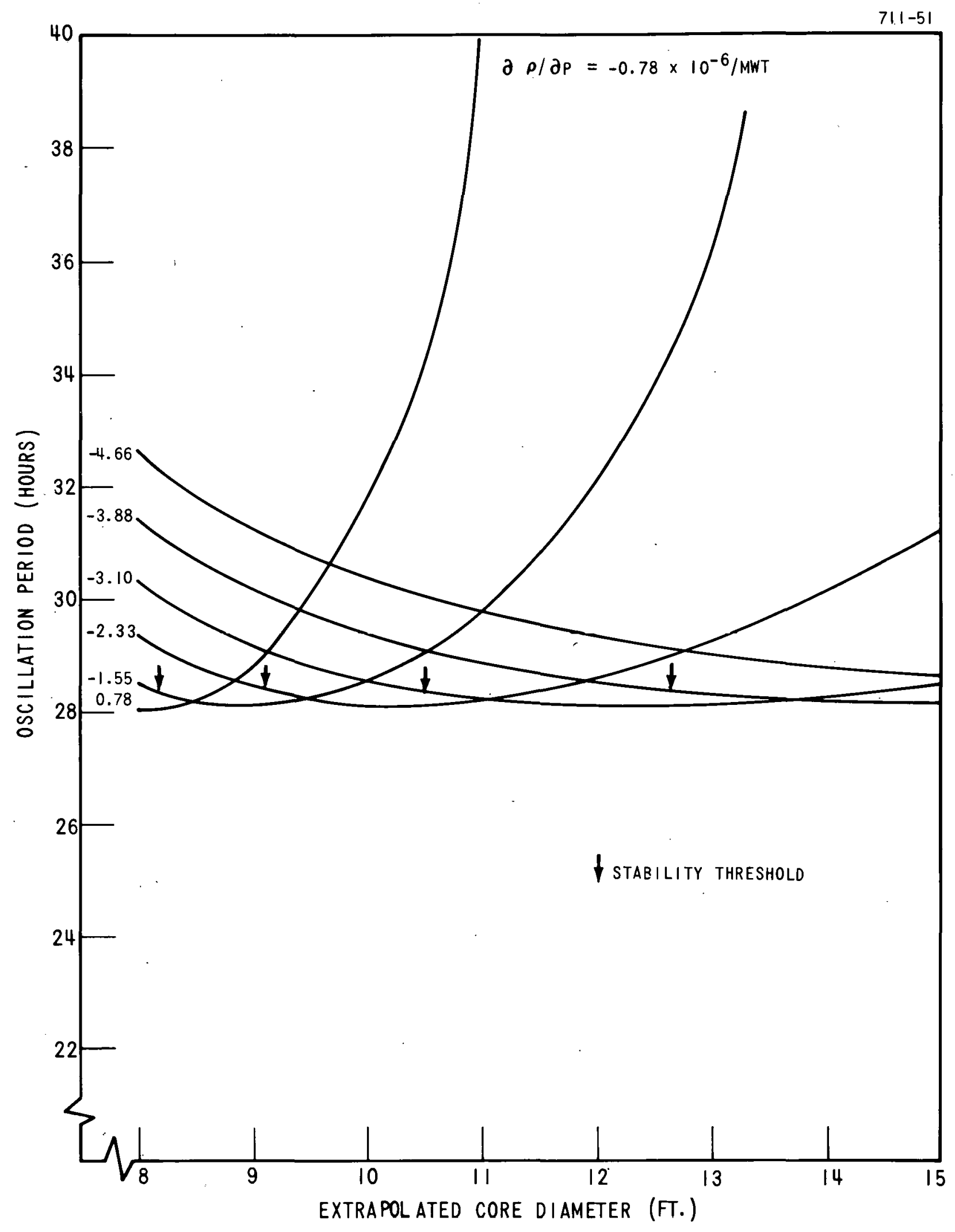

Figure 4-10. Variation of Oscillation Period with Core Diameter - Modal Analys is 


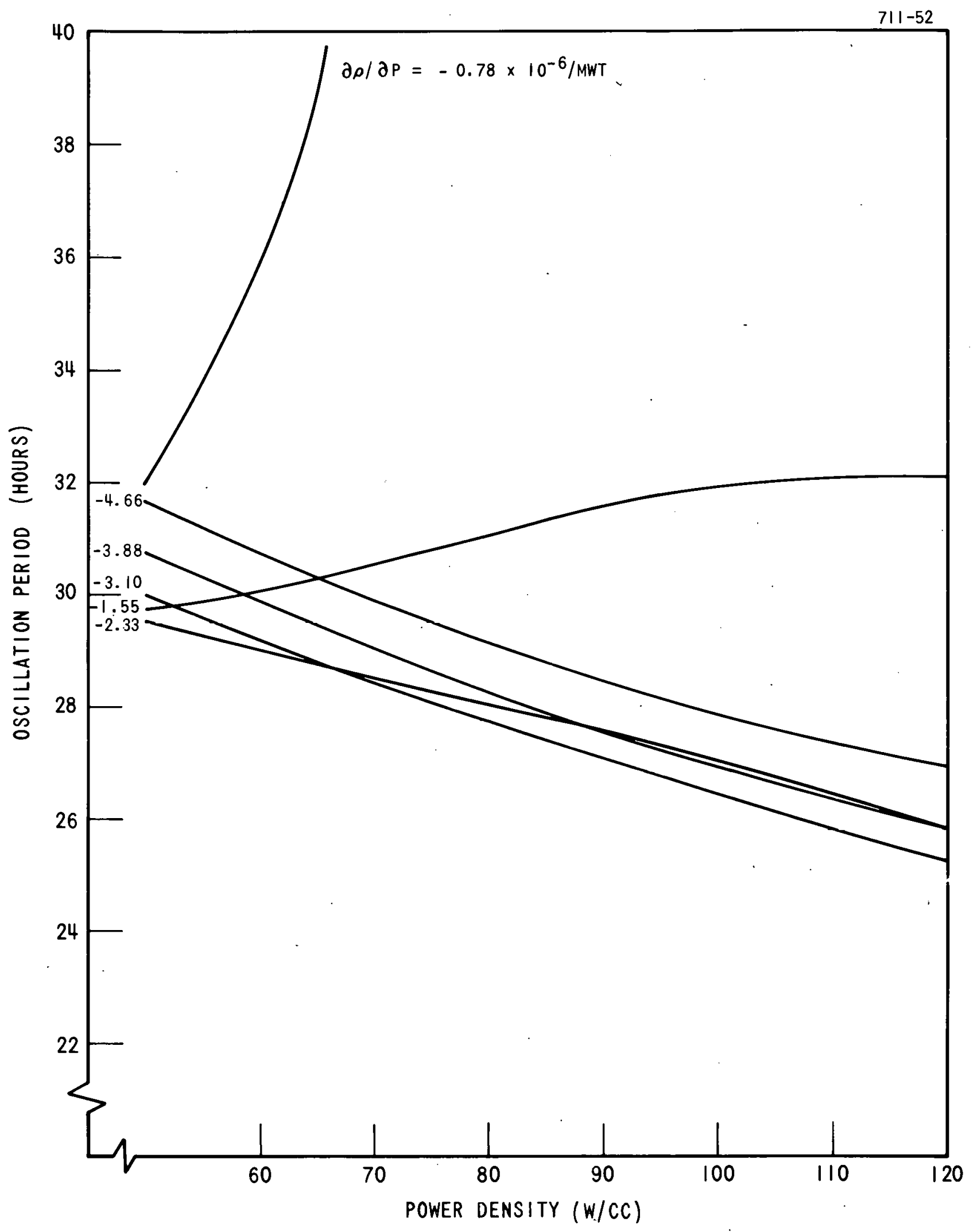

Figure 4-11. Variation of Oscillation Period with Power Density - Modal Analysis 


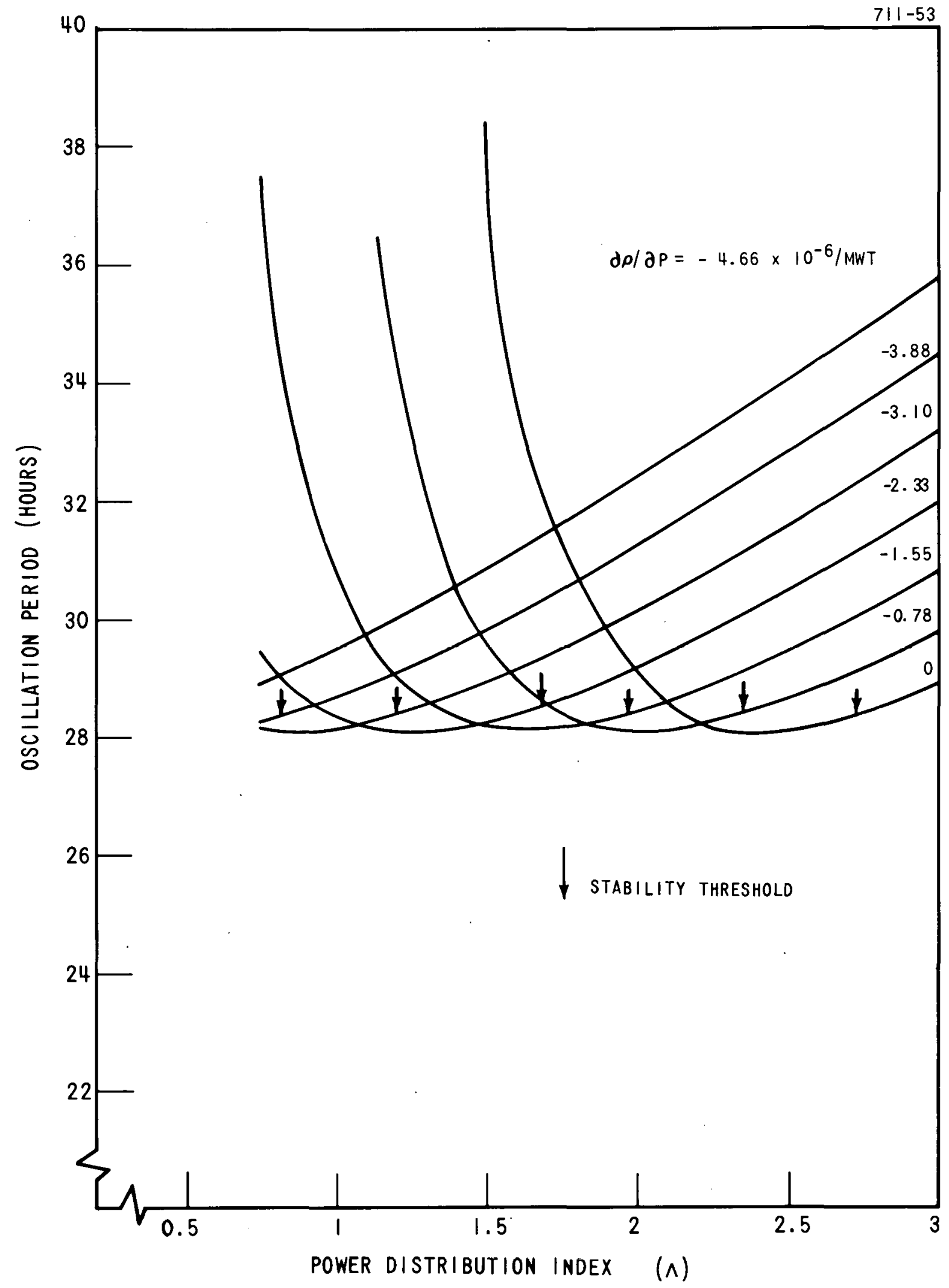

Figure 4-12. Variation of Oscillation Period with Power Distribution - Modal Analys is 


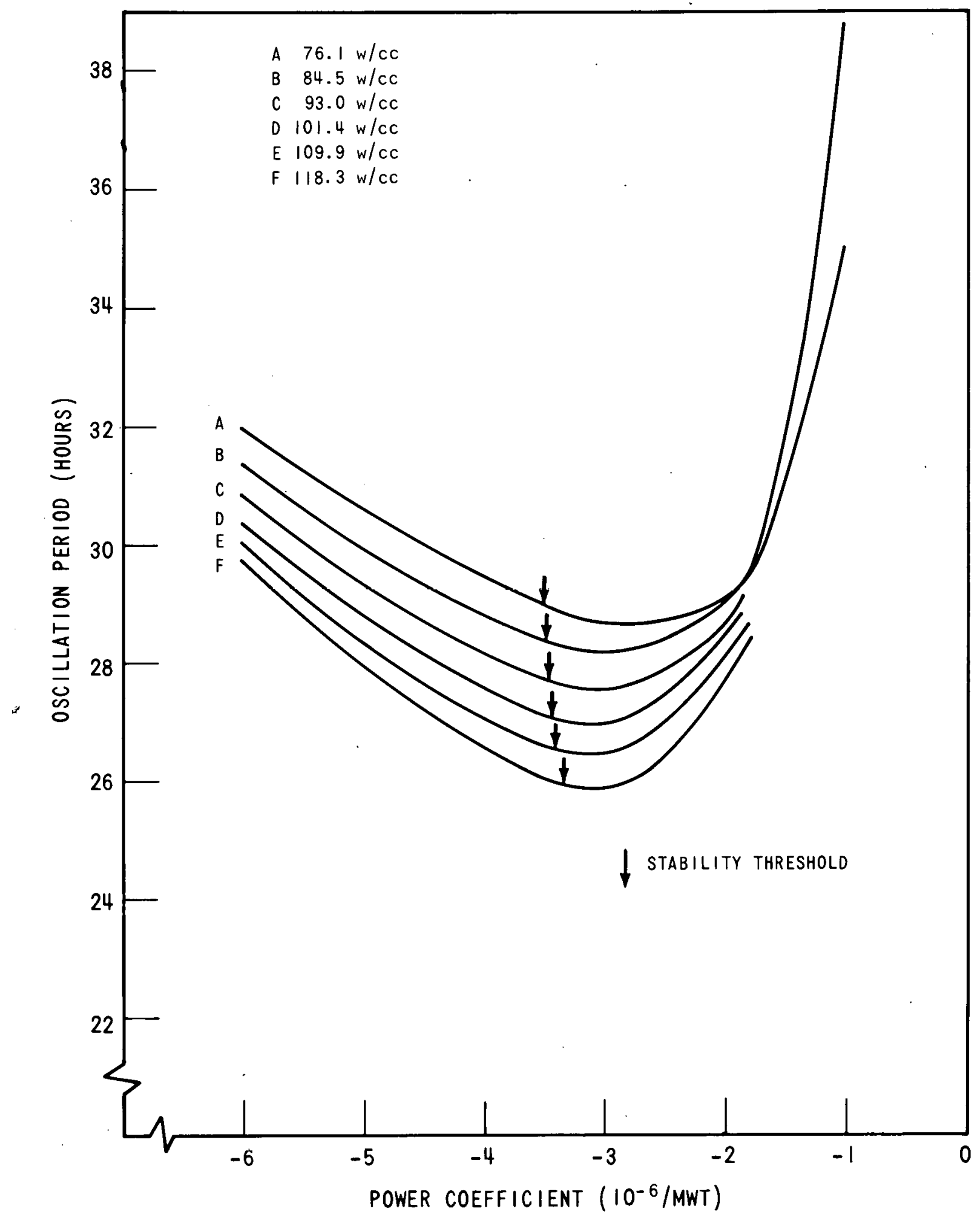

Figure 4-13. Variation of Oscillation Period with Power Coefficient -- Modal Analysis 


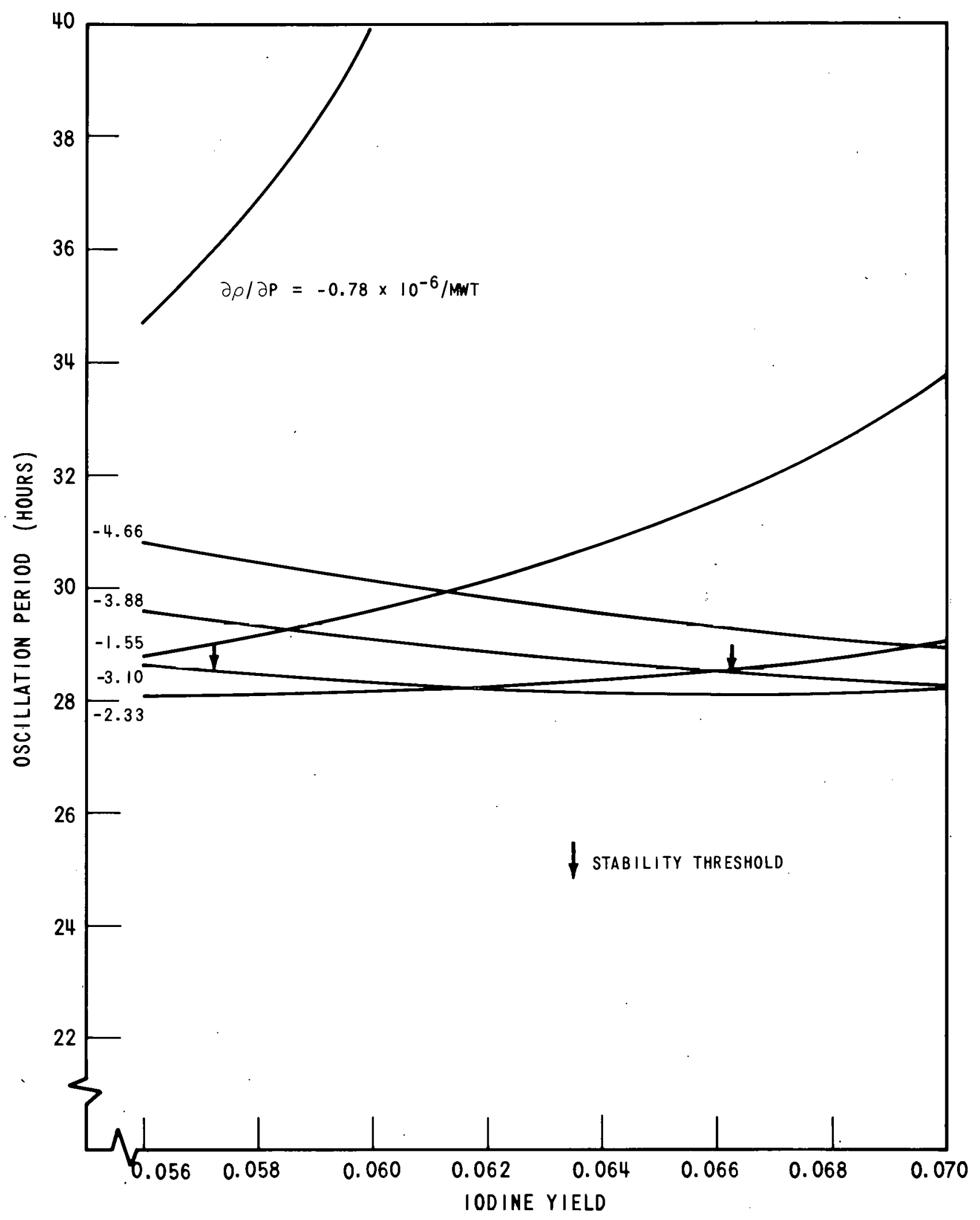

Figure 4-14. Variation of Oscillation Period with Iodine Yield - Modal Analysis 


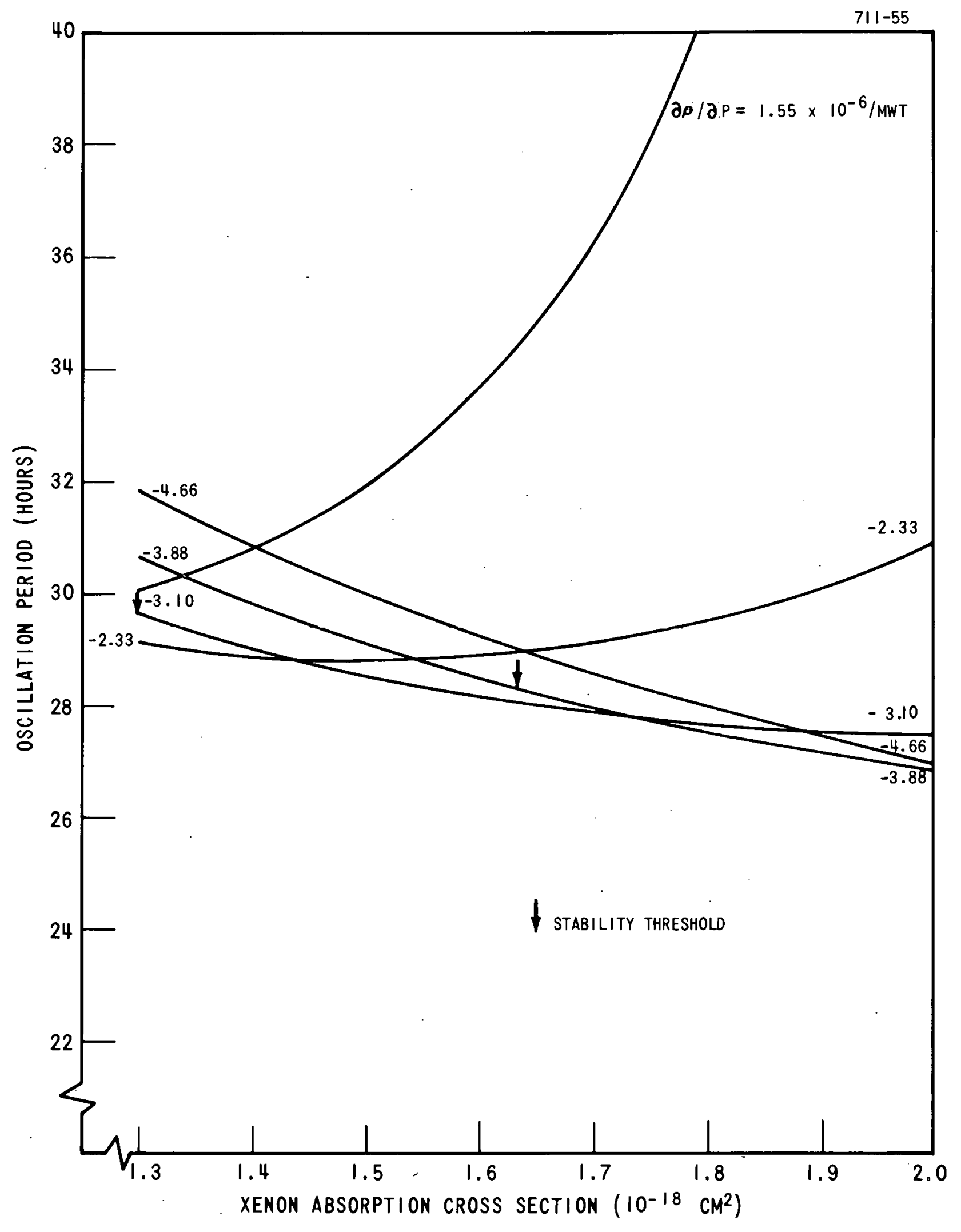

Figure 4-15. Variation of Oscillation Period with Xenon-Absorption Cross Section - Modal Analysis 


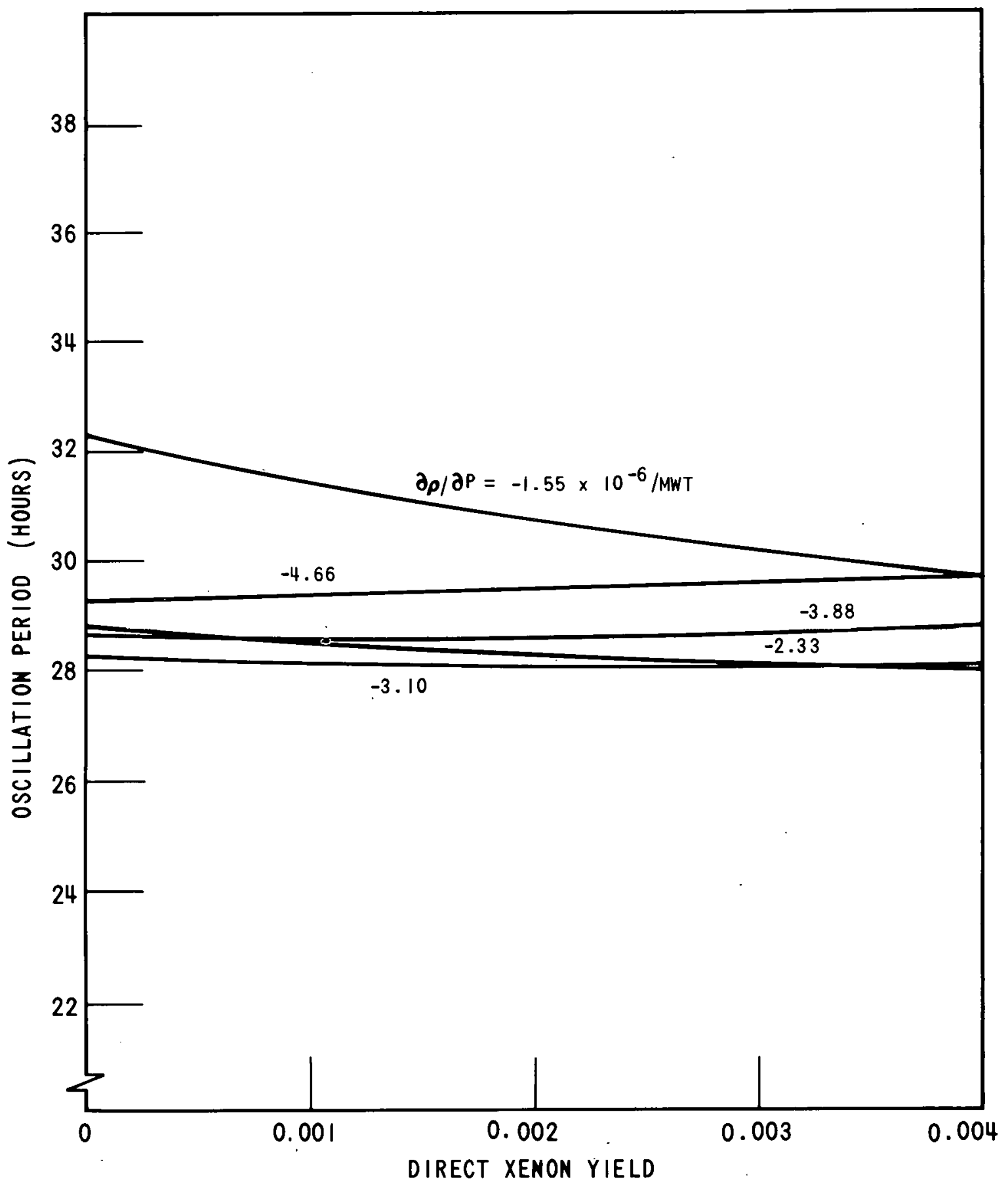

Figure 4-16. Variation of Oscillation Period with Direct Xenon Yield - Modal Analysis 


\subsection{NUMERICAL CALCULATIONS}

In Section 4.1 above, a modal analysis was performed in order to determine the sensitivity of core stability to a variety of physical parameters. From an analysis of that type, it is possible to obtain a broad picture of how a reactor will behave. Due to the approximate nature of the model employed, however, these results should be used more to provide a picture of trends than to give detailed results. In order to obtain a more exact picture of how a specific reactor will behave with variation of the above parameters and to obtain some measure of correlation between the modal and digital results, some parametric studies were performed with TURBO* and ZEST. Where possible, the value of the stability indices obtained from these calculations have been plotted beside the relevant curves (Figures 4-1 through 4-8) derived from the modal theory presented in Section 4.1.

\subsubsection{Effect of Variation of Power Density}

One of the known major influences affecting spatial stability is the reactor flux or power level. Calculations were made both with ZEST and TURBO* to numerically determine its effect.

In the two-dimensional TURBO* calculations, the power of an 11-foot core with an average enrichment of 2.6 percent and flattened power distribution was varied about its standard value by $\pm 1 / 3$ of this power. The size of the initial oscillations and, to a lesser extent, the subsequent growth rate were found to be sensitive to this parameter. Since the cores at full power ( 84.5 watts/ cc) and at $4 / 3$ full power were quite divergent, it was not possible to calculate reasonable stability indices. The curves, however, are shown in Figures 4-17 through 4-19.

For the one-dimensional ZEST analysis, an 11-foot core of average enrichment 2.6 percent and flattened power distribution was used. The power densitities for the calculations were $70,84.5$ and 100 watts/cc. The time-step corrected stability indices for those curves are compared in Figure 4-3 to the results of the modal analysis. As can be seen from the figure, the points do not fit any curve accurately. One reason for the discrepancy lies in the fact that since the power coefficient is a function of power level, each point should lie on a slightly different parametric curve. 


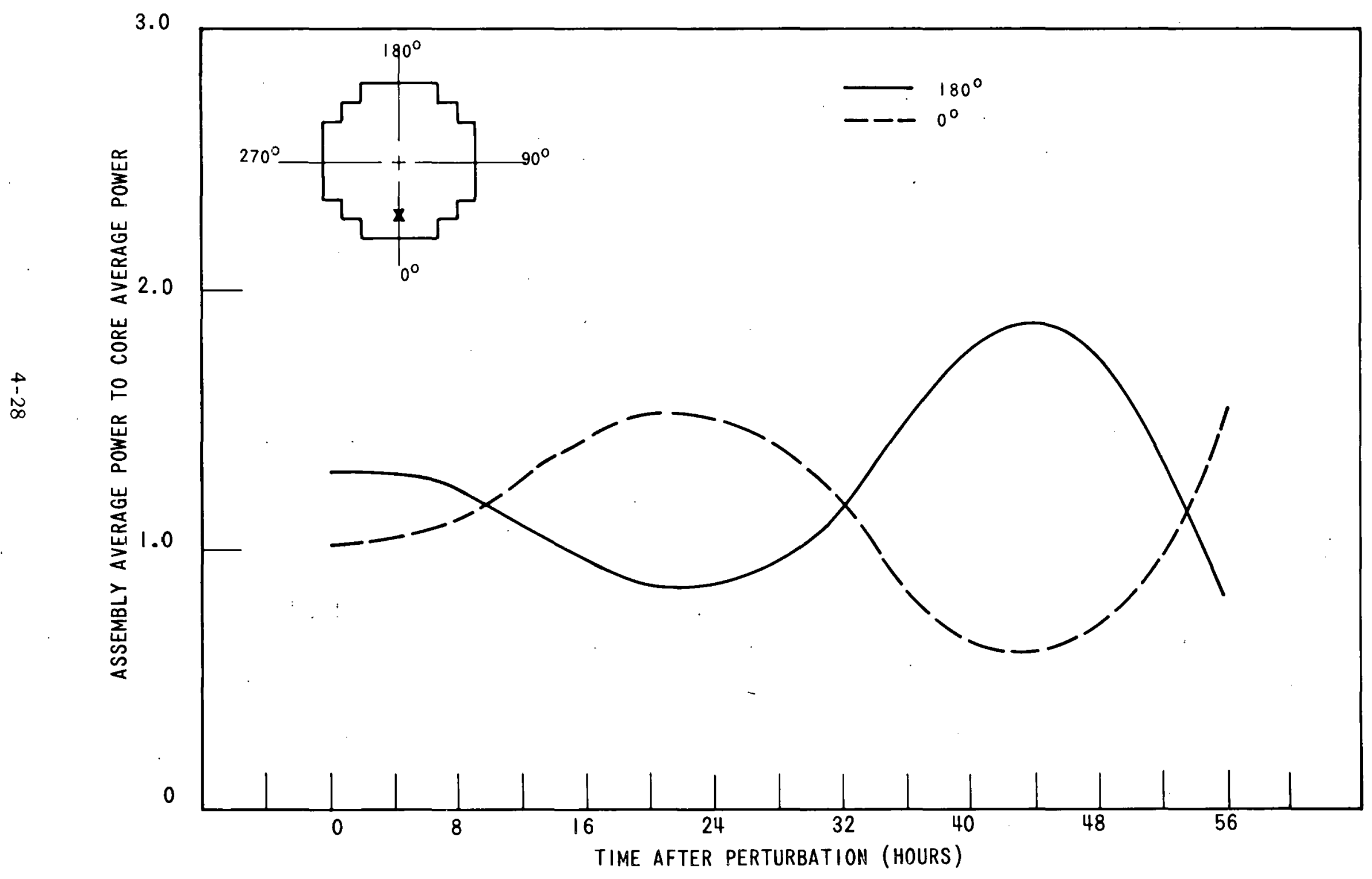

Figure 4-17. Xenon-Induced Oscillations at, 2/3 Power 


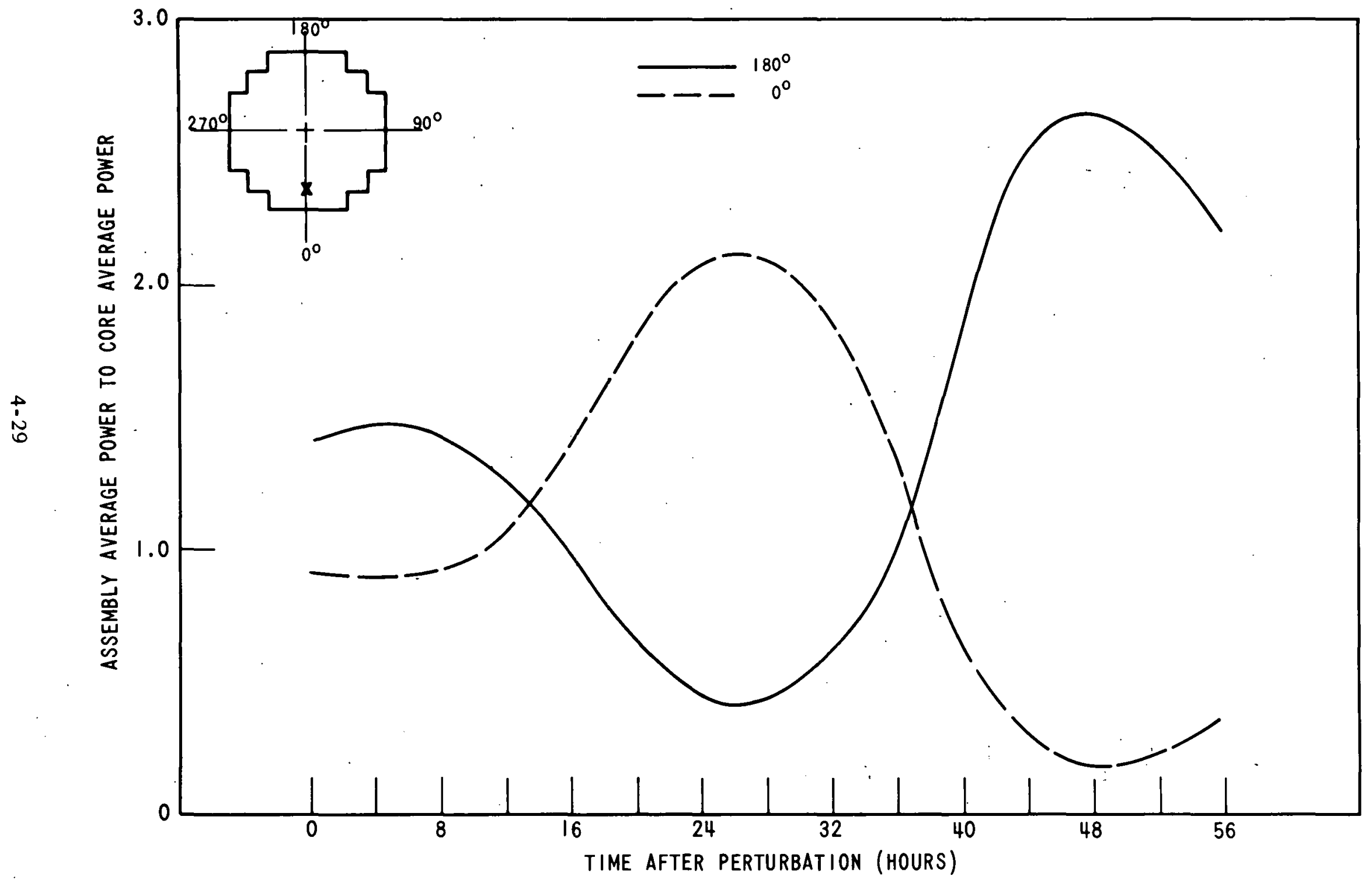

Figure 4-18. Xenon-Induced Oscillations at Full Power 


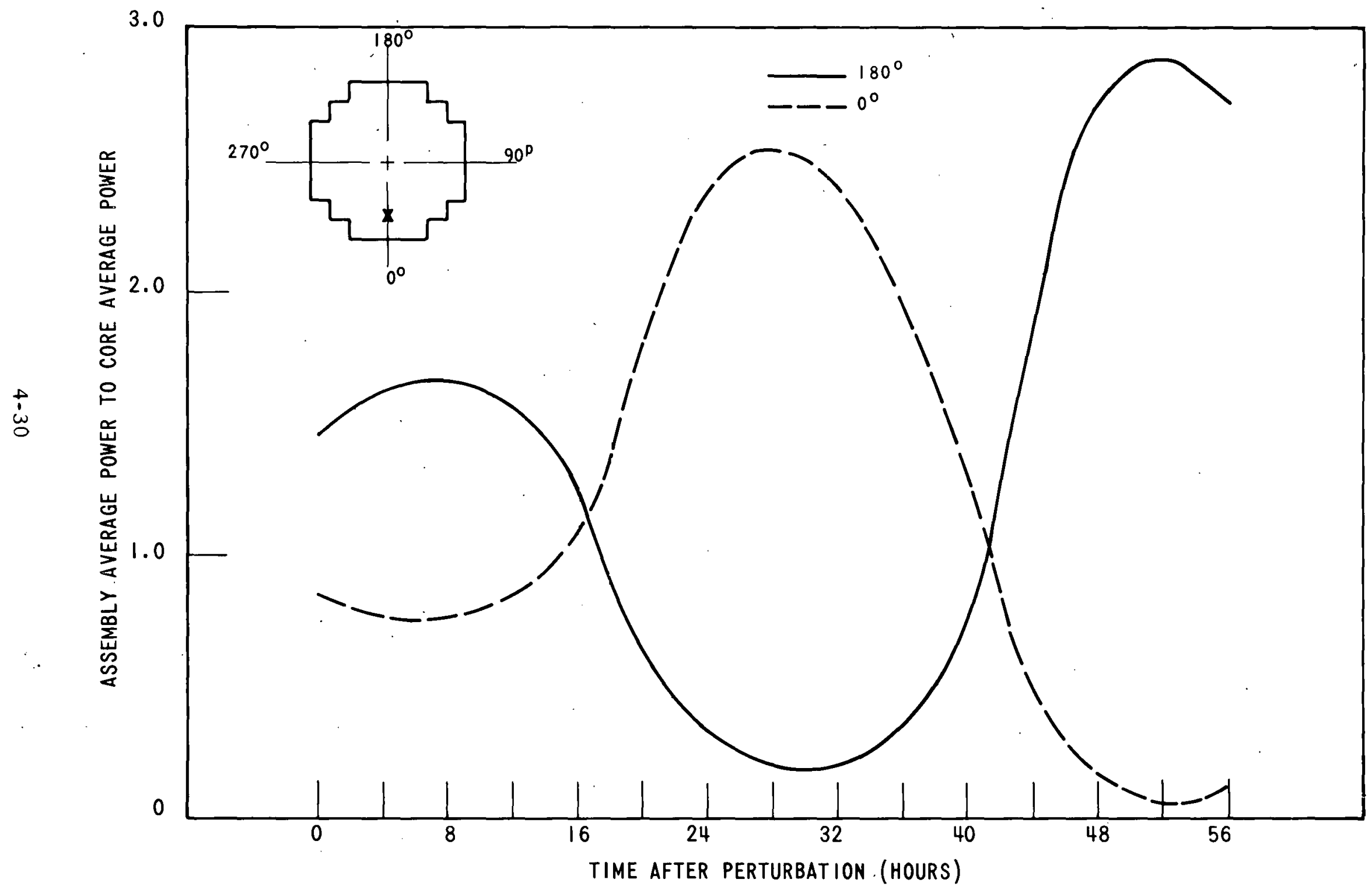

Figure 4-19. Xenon-Induced Oscillations at 4/3 Power 


\subsubsection{Effect of the Equilibrium Power Distribution}

The spatial stability characteristics of the core are very sensitive to the initial unperturbed power distribution since leakage and coupling between regions are strongly dependent on the initial flux shape. In a given core size fuel management requirements lead to varying power distributions from the initial core loading to achievement of the equilibrium cycle. Moreover, design optimizations naturally require flattened power distributions, which tend to decrease the stability characteristics of a core. It is important to evaluate the sensitivity of xenon-induced azimuthal oscillations to power distributions in the $x-y$ plane.

Three types of initial power distribution were examined with reference to spatial oscillations. These were:

1. dished, flattened and cosine distributions;

2. triple-peak distributions (i.e., a dished distribution with an added peak in the center);

3. checkerboard distribution.

For all of these, an 11-foot core was used. Approximately the same average enrichment ( 2.6 percent) was maintained in all cases and the cores were operated at the same power density ( 84.5 watts/cc).

In case (1) above three-zone enrichments were selected to yield $x-y$ power distributions ranging from a distribution peaked at the center (batch loading) to a power distribution dished in the center of the core. These initial power distributions can be seen in Figure 4-20. All cores were perturbed in the same fashion as described in Section 3.1 and the resulting oscillations for the equilibrium power distribution A, B, C and D of Figure 4-20 are shown in Figures 4-21, 4-22, 4-18 and 4-23. These figures show the power oscillations at the location of the perturbation and at the point symmetrically opposite along the $0^{\circ}-180^{\circ}$ axis. Temperature feedbacks were not accounted for in these calculations. Figure 4-21 shows that in the batch-loaded core, the oscillations are convergent and of relatively small magnitude. The oscillations are divergent for the other three cases and increase in magnitude from case $B$ to case D. For case D, non-1inear effects introduce large distortions in the oscillations. 


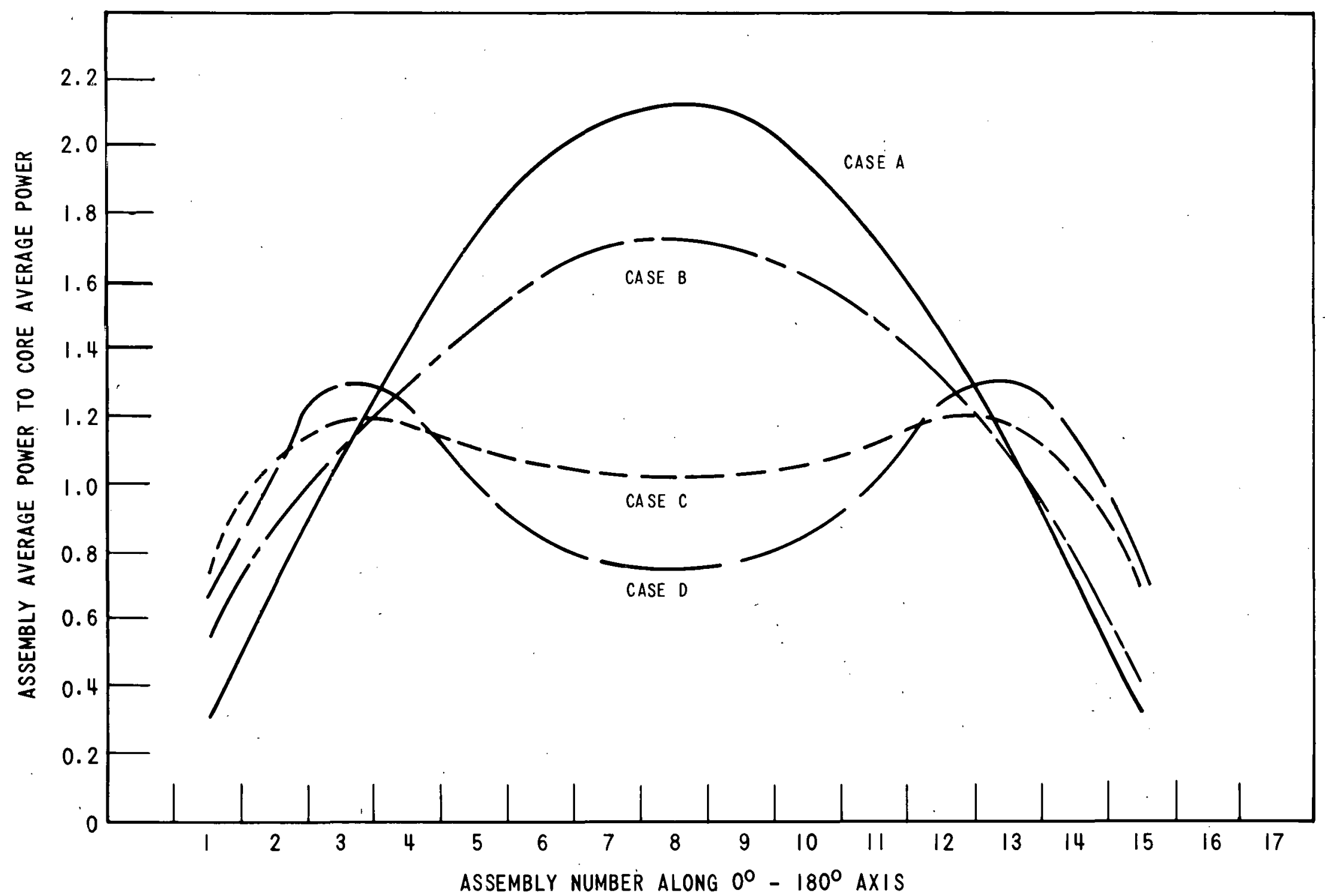

Figure 4-20. Steady-State Poner Distribution in 11-Foot Diameter Core 


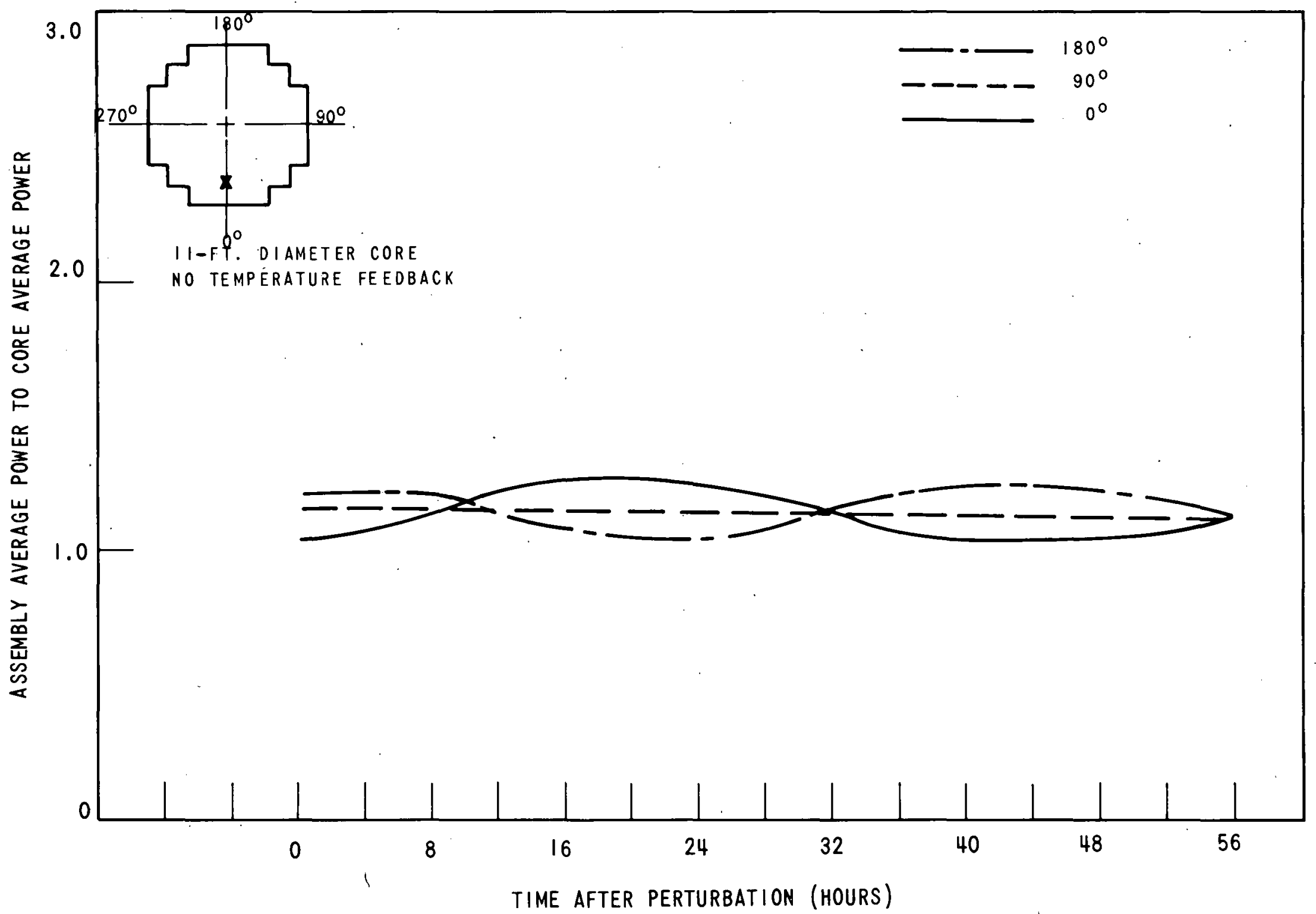

Figure 4-21. Effect of Initial Power Distribution on Xenon-Induced Oscillations - Case A 


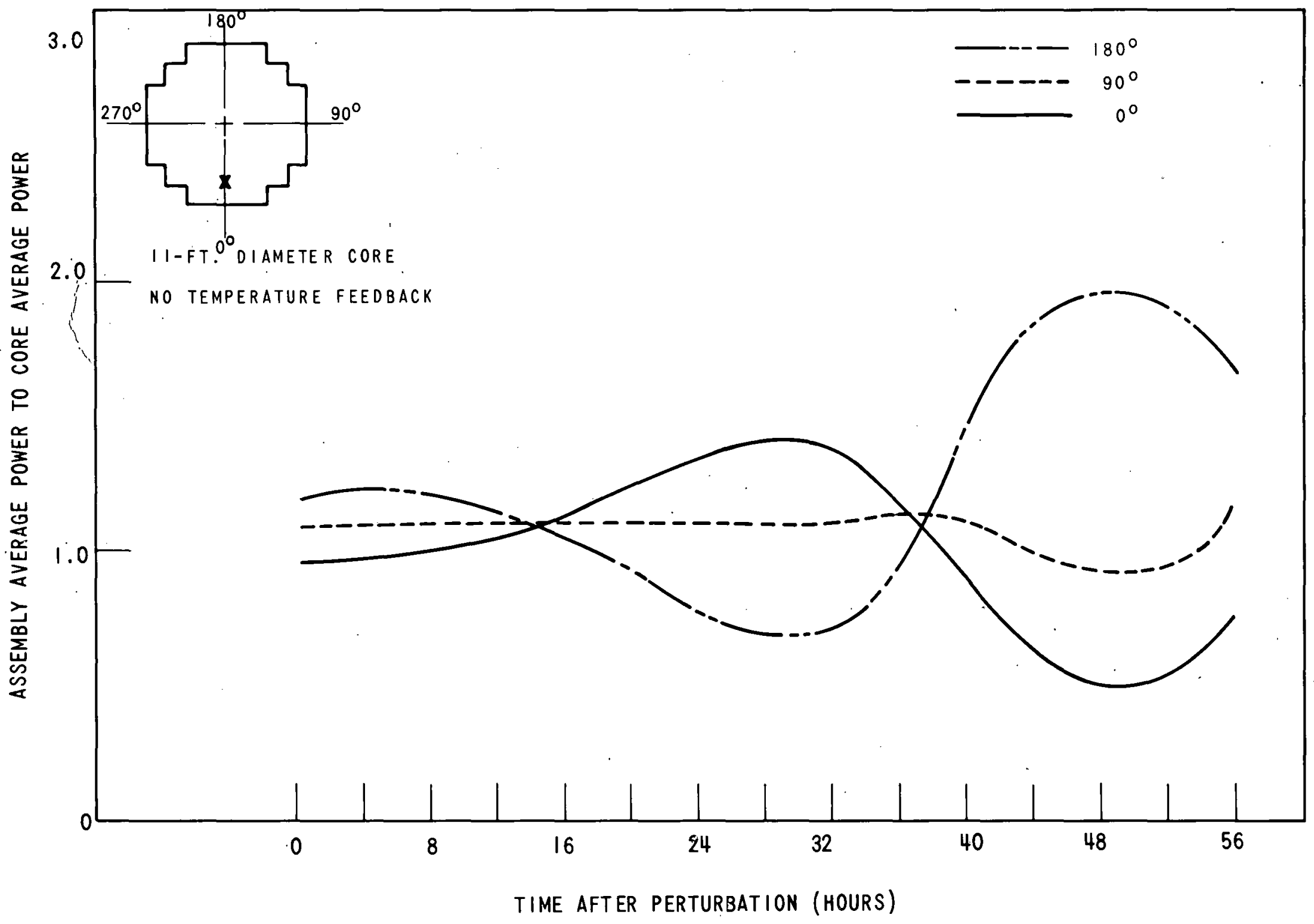

Figure 4-22. Effect of Initial Power Distribution on Xenon-Induced Oscillations - Case B 


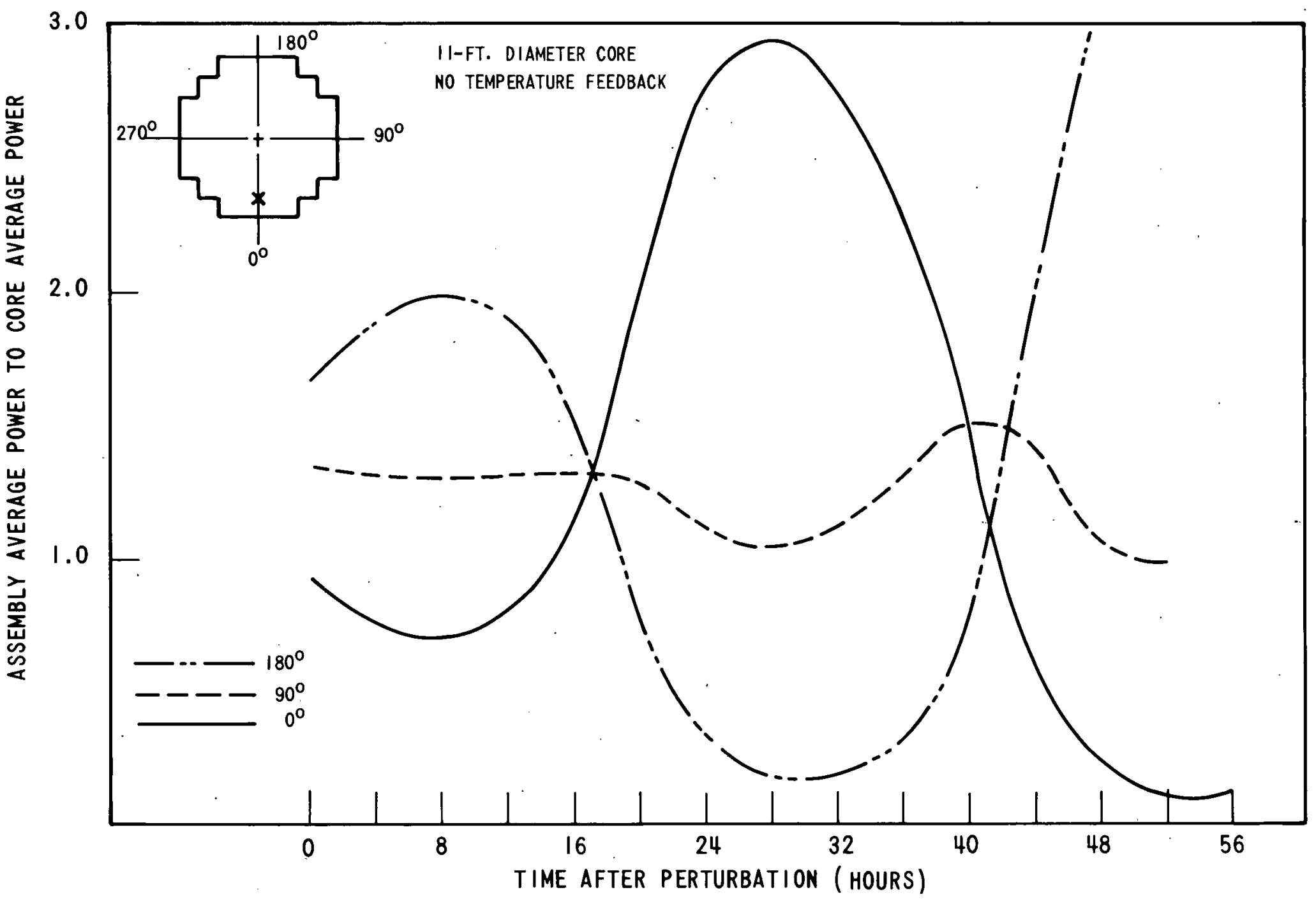

Figure 4-23. The Effect of Initial Power Distribution on Xenon-Induced Oscilliations - Case D 
The unperturbed power distributions for the two 11-foot triple peak cores (case 2 above) can be seen in Figures 3-12 and 3-13. The subsequent oscillations for the first of these are considerably weaker than the latter, as its initial power distribution is much closer to a cosine curve. These responses of the power in time can be seen in Figures 4-24 and 4-25.

As with the triple peak cores, the spatial distribution of the checkerboard core (case 3 above) was described in section 3.1.2. The resulting power distribution from the checkerboard core does not differ greatly from the corresponding conventionally-enriched core although it exhibits discontinuities in power at the assembly interfaces. Figure 4-26 shows the power oscillations in two adjacent assemblies, graphs $A$ and $A^{\prime}$ corresponding to assemblies of enrichment 2.6 percent while graphs $B$ and $B$ ' correspond to assemblies of enrichment 2.1. These can be compared with the equivalent plot of the oscillations for the conventionally-zoned core [Figure (4-18)] and it would appear that stability is not noticeably affected by using a checkerboard arrangement.

The above results show that the spatial stability characteristics of a given size core are, in general, sensitive to the unperturbed power distribution in the $x-y$ plane. Thus, one would expect the stability characteristics of a core to vary with plant lifetime, with the most unstable conditions occurring at the beginning of each fuel cycle. Because of the relatively long periods associated with xenon-induced oscillations, and in view of the success achieved in controlling axial xenon oscillations, [5] no major problem is anticipated in the control of azimuthal xenon oscillations.

\subsubsection{Effect of Core Size}

As the linear dimensions of a nuclear system increase, leakage from the core decreases and coupling between the regions of the core is also decreased. The efficiency of neutron leakage to counteract a local change in multiplication due to a local perturbation decreases as core size increases, thus the inherent instability to spatial xenon oscillations is enhanced. Linearized perturbation analyses lead to threshold criteria when the oscillations are small. Quantitative data, however, can only be obtained for 


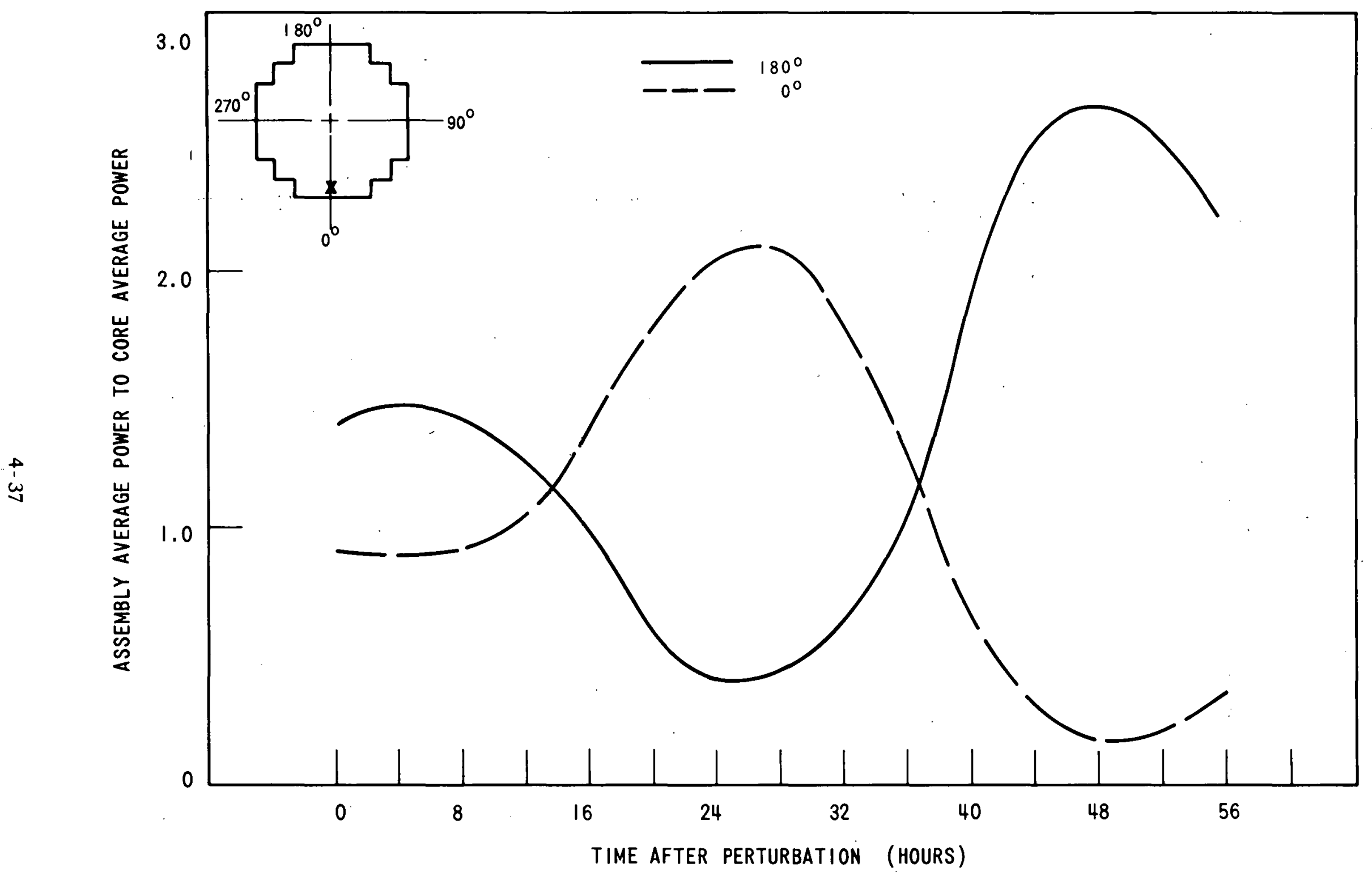

Figure 4-24. Xenon-Induced Oscillations for Triple Peak Core Number 1 


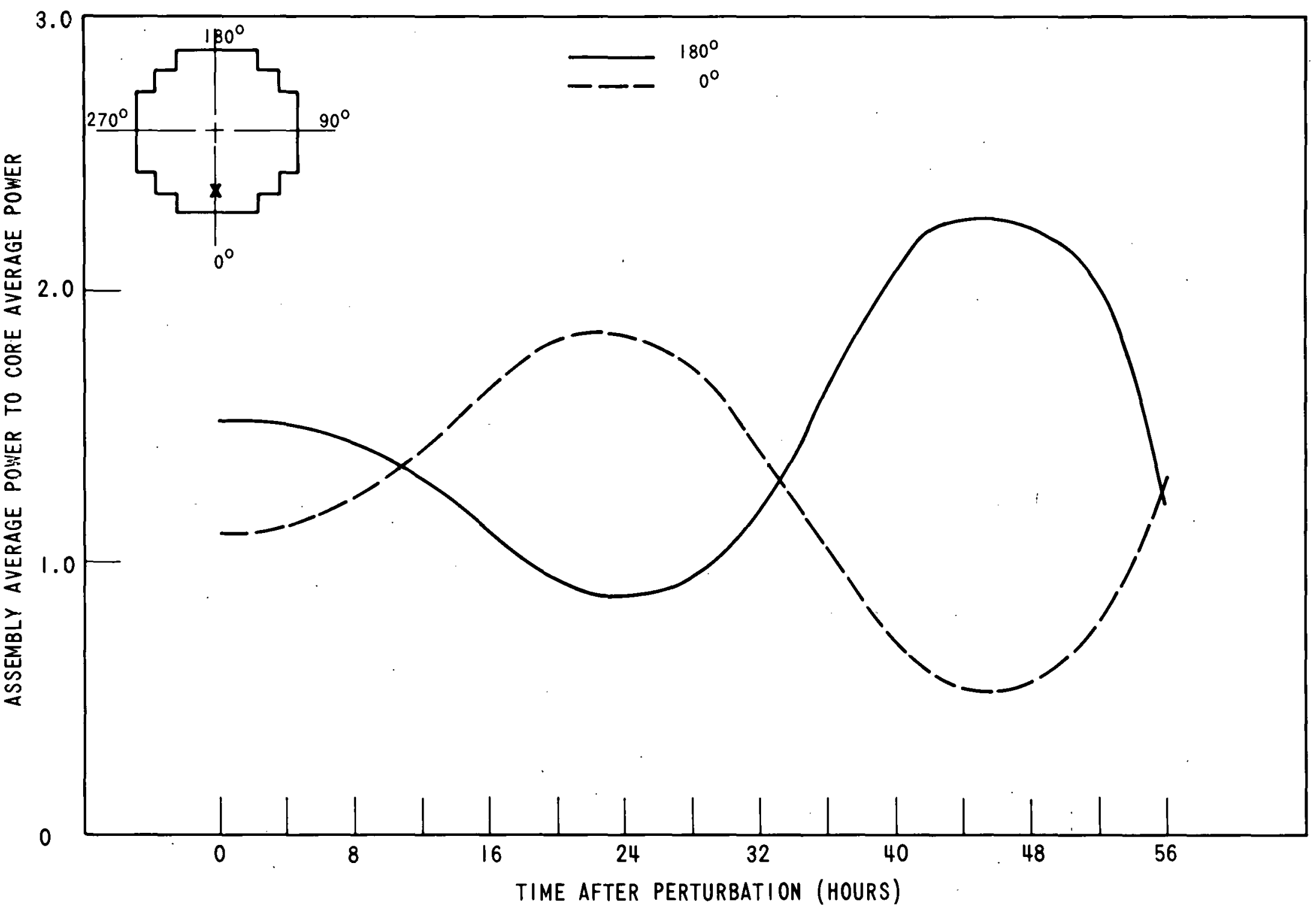

Figure 4-25. Xenon-Induced Oscillations for Triple Peak Core Number 2 


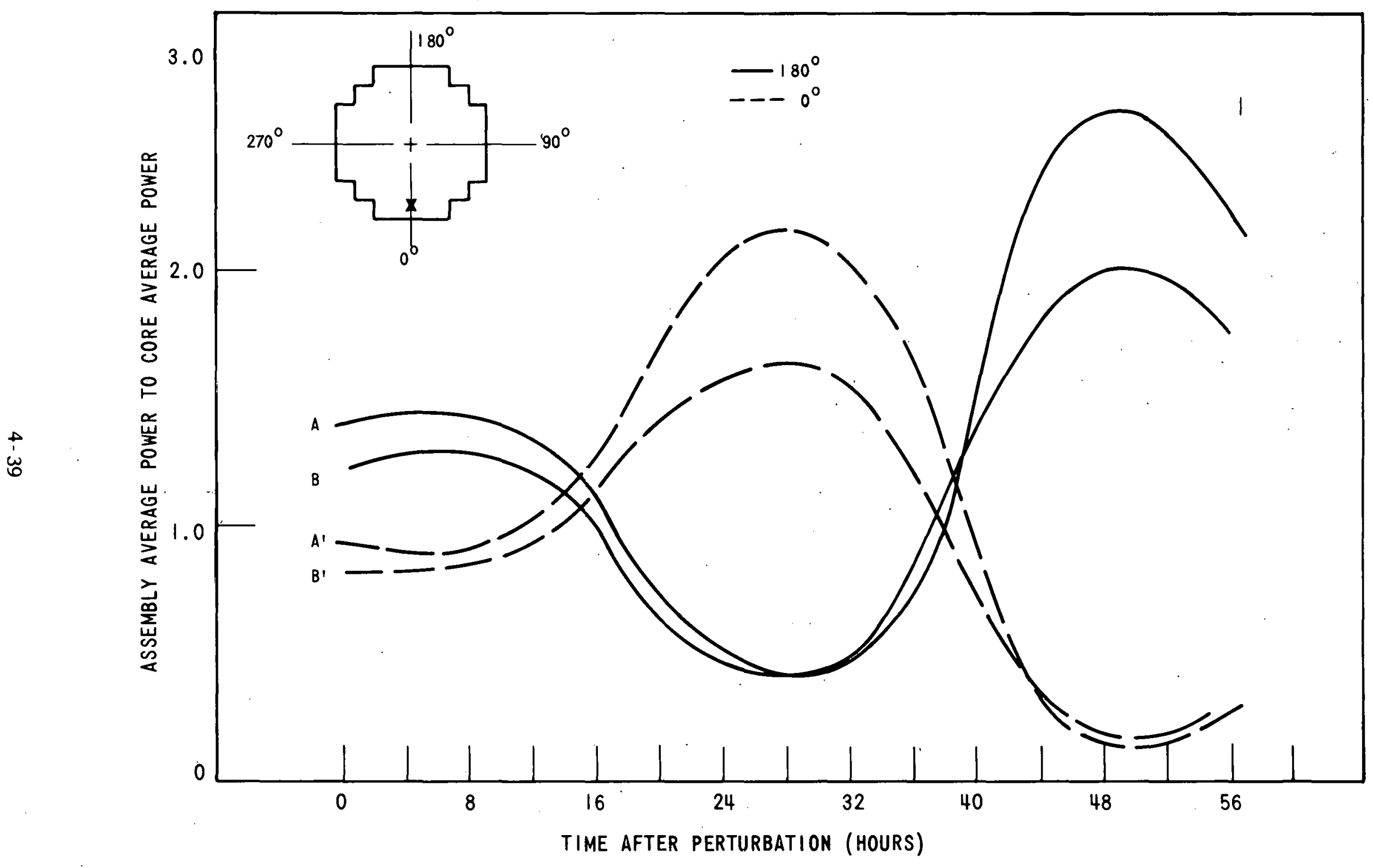

Figure 4-26. Xenon-Induced Oscillations for Checkerboard Core 
idealized geometries and fuel loadings. Although PWR cores approximate a right circular cylinder, they exhibit a non-circular, staggered boundary in the $x-y$ plane, with non-uniform zoning inside the boundary. The stability characteristics and the nature of spatial oscillations in such a system are best studied through actual simulation using multidimensional diffusion codes. Calculations were performed with TURBO* in $x-y$ geometry for a number of core sizes varying from approximately 8 to 15 feet in equivalent diameter. Core configurations were selected to correspond to actual PWR designs and, in the case of the very large.cores, to anticipated designs. Since the spatial stability characteristics of a core are sensitive to the steady-state, unperturbed flux distribution (refer to Section 4.2.2), three-zone enrichments were selected to yield an approximately flat distribution in all cores, so that the effect of size only on xenon-induced azimuthal oscillations could be studied. The average enrichment for all cores was approximately 2.6 percent, and the same power density was used $(84.5 \mathrm{w} / \mathrm{cc})$ in all cases, thus resulting in approximately the same flux level for all sizes.

Table 4-1 lists various core data used in the analysis. All cores had the same size of assembly ( 8.5 in. $x 8.5$ in.). The last column in the table lists the maximum ratio of assembly-average power to core-average power for the steady-state unperturbed distribution. This column gives a measure of how much the distribution deviates from flatness.

Figures 4-27 through 4-30 show the resulting oscillations as assembly-average power to core-average power for three core locations (0,90, and 180 degrees) for four of the cores described in Table 4-1. It is to be stressed that the effects of temperature feedback were not included in these calculations and consequently the spatial oscillations which were observed do not correspond to actual core operation. It should also be noted that because of the timestep effect (refer to Section 2.3.2), the oscillations which were particularly divergent will, in this context, have their stability index substantially underestimated. Nevertheless, these calculations clearly exhibit the effect of core size on xenon-induced azimuthal instabilities.

Figure 4-27 shows that the 8.8-foot diameter core with a flat power equilibrium power distribution is close to the stability threshold for azimuthal 


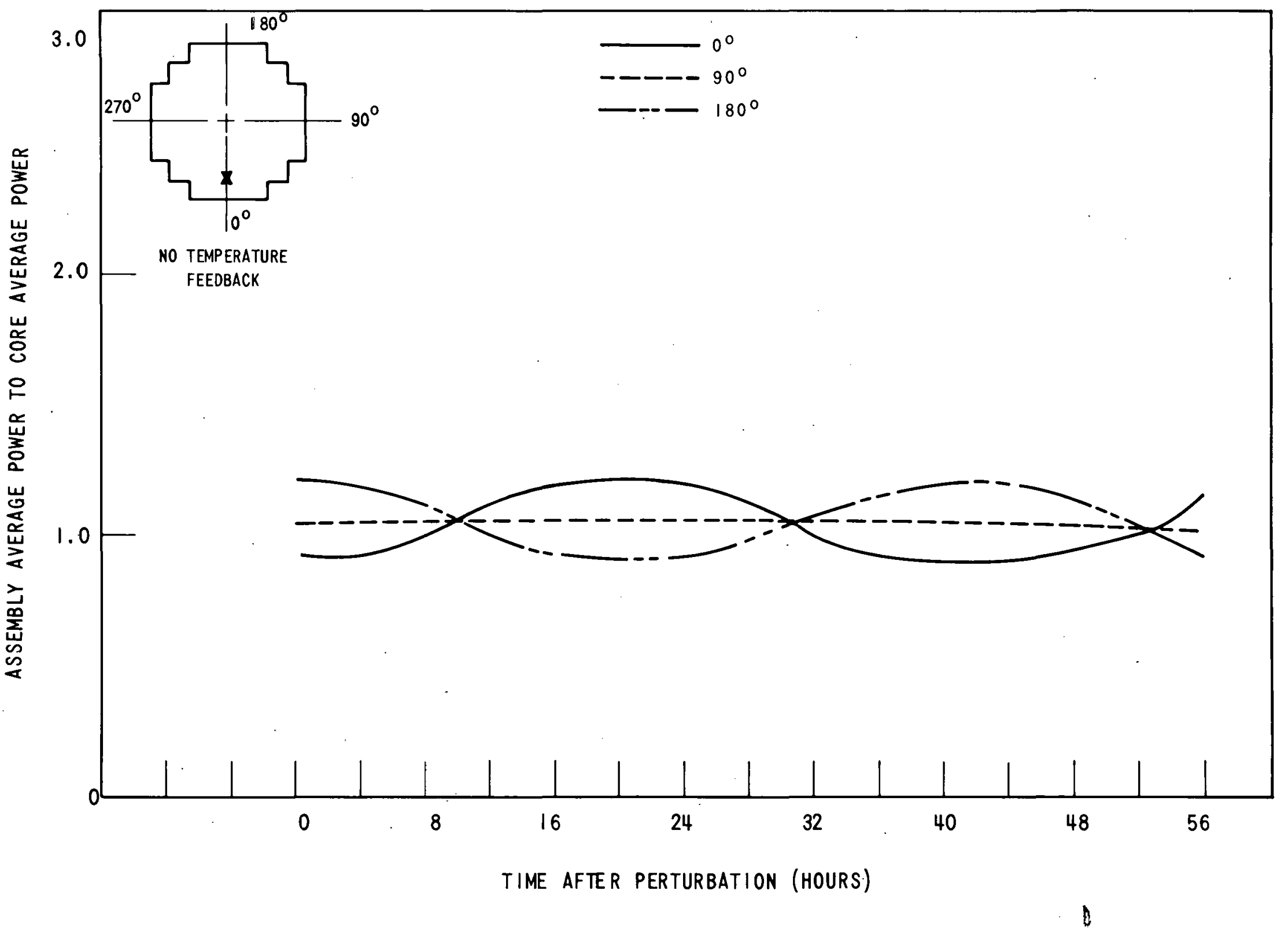

Figure 4-27. Xenon-Induced Oscillations for 9-Foot Core 


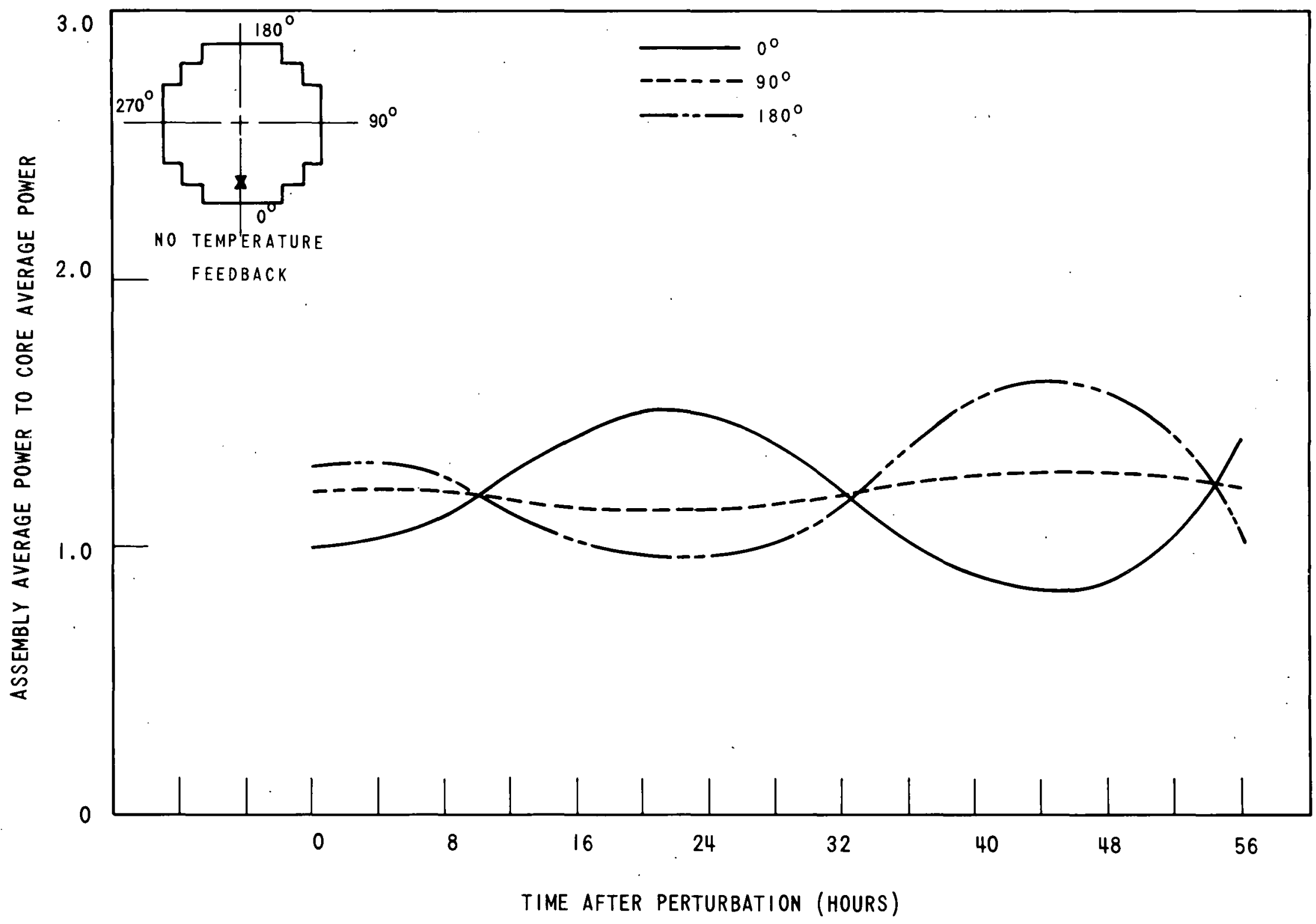

Figure 4-28. Xenon-Induced Oscillations -for 10-Foot . Core 


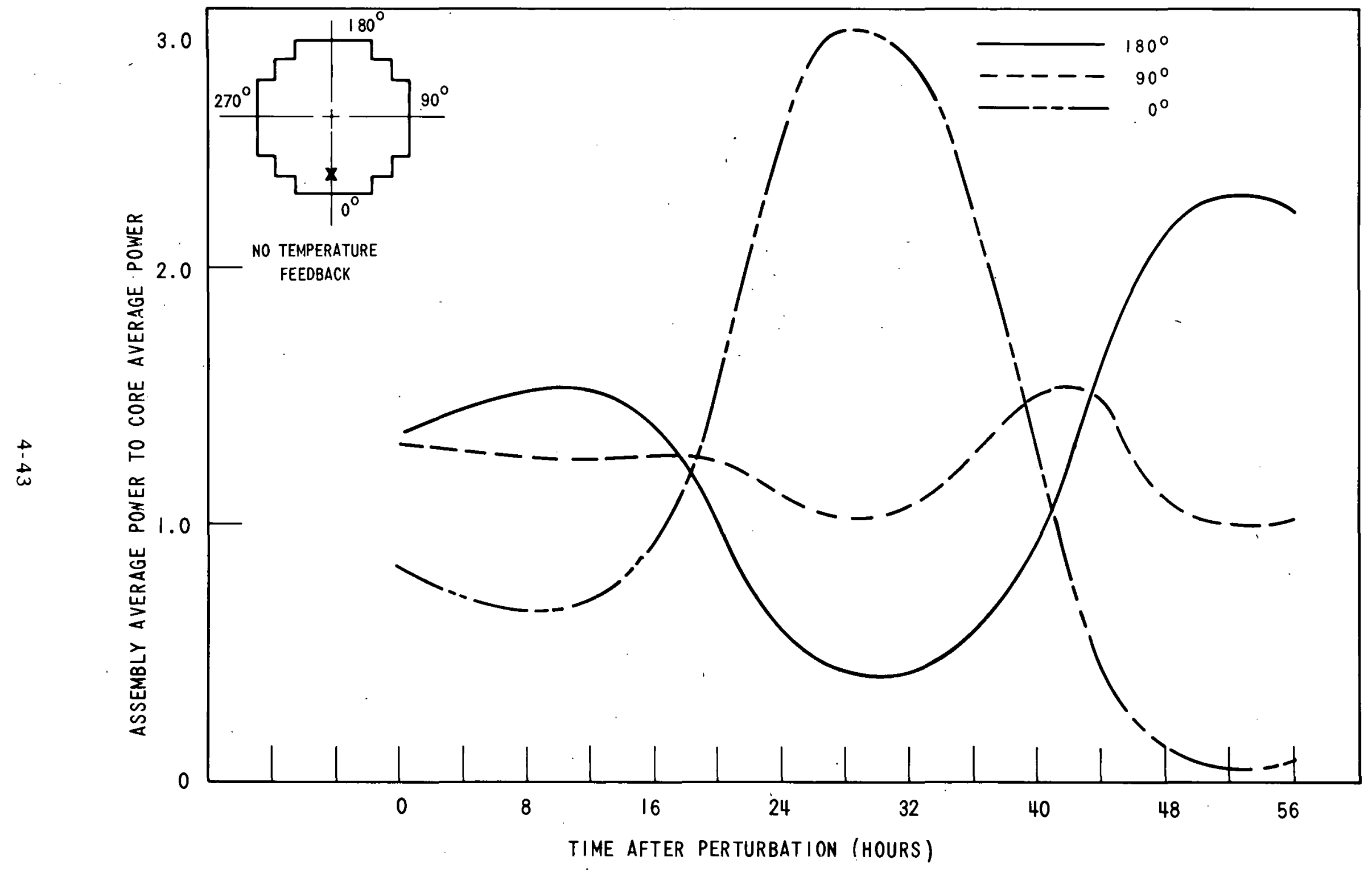

Figure 4-29 Xenon-Induced Oscillations for 13-Foot Core 


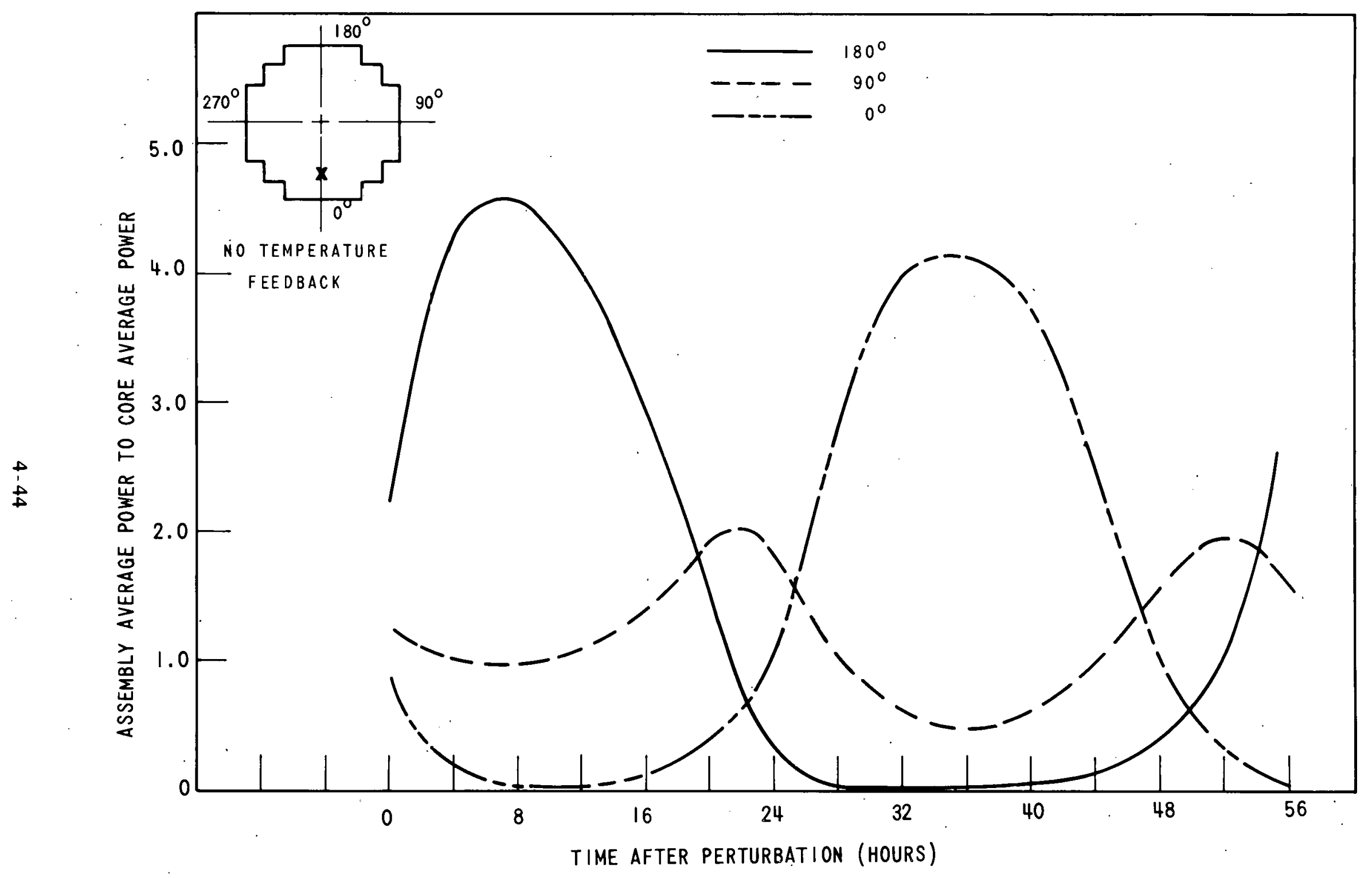

Figure 4-30. Xenon-Induced Oscillations for 15-Foot Core 
oscillations. However, if this threshold is modified in light of the time-step length error (Section 2.3.2) the core on the threshold is now found to be 8.5 feet in diameter. Again it should be remembered that the strongly stabilizing effect of temperature feedback is not included here.

TABLE 4-1

CORE ANALYSIS DATA

\begin{tabular}{|c|c|c|c|c|}
\hline Case & $\begin{array}{c}\text { Number of } \\
\text { Assemblies }\end{array}$ & $\begin{array}{c}\text { Equivalent Core } \\
\text { Diameter (ft) }\end{array}$ & $\begin{array}{c}\text { Three-Zone } \\
\text { Enrichment }\end{array}$ & $\left(\begin{array}{l}\overline{\mathrm{P}}_{\text {assembly }} \\
\overline{\mathrm{P}}_{\text {core }}\end{array}\right)_{\max }$ \\
\hline 1 & 121 & 8.8 & $2.3 / 2.6 / 3.0$ & 1.30 \\
2 & 157 & 10.0 & $2.4 / 2.6 / 2.9$ & 1.32 \\
3 & 193 & 11.0 & $2.4 / 2.5 / 3.0$ & 1.22 \\
4 & 269 & 13.1 & $2.5 / 2.6 / 2.9$ & 1.30 \\
5 & 349 & 14.9 & $2.5 / 2.6 / 2.7$ & 1.39 \\
\hline
\end{tabular}

Stability threshold calculations based upon the Randall and St. John perturbation modal analysis [21] predict that, with a power coefficient of $-3.1 \times 10^{-6} /$ MWt a 10.5 -foot core would be on the threshold for azimuthal oscillations in the first harmonic. This was obtained by associating a factor (1/1.5) with the difference in eigenvalue between the first and fundamental mode to account for flux flattening. The threshold can be seen on Figure 4-2 which shows the sensitivity of the stability index to core length according to modal theory.

Two calculations were also performed with ZEST, one with a 10-foot core and one with an 11-foot core. The time-step-corrected stability indices for these two cases are plotted for comparison with modal. curves on Figure 4-2 and it appears that they are in substantial agreement. It is pointed out, however, that there is a large degree of uncertainty in the stability indices, particularly so in those derived from the modal analysis, because of the inherent approximations. This is borne out in the fact that the TURBO* predicts a noticeably smaller core for the stability threshold than does the modal theory. 
Table 4-2 lists the periods and time-step corrected stability indices for various sizes of core. The slight increase in oscillation period with core size agrees qualitatively with simple modal analysis. This effect can be observed by examining Figure 4-10 where it can be seen that up to about a 12-foot core, increasing the core size decreases the period. For the 11-foot core, the oscillations quickly become large and non-linear effects, such as flux saturation, become important. This saturation precludes the use of the stability index as a meaningful quantity. Thus, in Table 4-2 below, it is omitted where saturation is appreciable.

TABLE $4-2$

EFFECT OF CORE SIZE ON PERIOD AND STABILITY INDEX

\begin{tabular}{|l|c|c|c|c|}
\hline Case & $\begin{array}{c}\text { Equivalent Core } \\
\text { Diameter (ft) }\end{array}$ & $\begin{array}{c}\text { Oscillation } \\
\text { Period (hrs) }\end{array}$ & $\begin{array}{c}\text { Stability } \\
\text { Index (hrs }\end{array}$ & Code \\
\hline 1 & 8.8 & 41 & +0.041 & TURBO* \\
2 & 10.0 & 43 & -- & TURBO* \\
3 & 11.0 & 46 & -- & TURBO* \\
4 & 10.0 & 35 & -0.004 & ZEST \\
5 & 10.6 & 35 & +0.015 & ZEST \\
\hline
\end{tabular}

\subsubsection{Effect of Varying Certain Core Parameters}

Due to approximations in theory and inaccuracies in basic physical data, results of calculations may have some degree of uncertainty in their value. To determine the sensitivity of results to changes in the relevant parameters, calculations were performed with ZEST and TURBO* in which these parameters were varied about their mean value and the solutions compared. The parameters investigated were:

1. thermal absorption cross section of Xe-135;

2. total yield from fission;

3. direct xenon yield from fission.

The Xe-135 absorption cross section and the iodine yield were varied by \pm 10 percent and the direct xenon yield was changed from 0.002 to 0.00 and to 0.004 . 
Cases (1) and (2) above were investigated particularly because the values given to these quantities will vary over the lifetime of the core. In the first case, the microscopic absorption cross section will change because of spectrum variations in the core with time. The total yield will also change with time because of the buildup of plutonium.

The direct xenon yield has a stablizing influence, since it contributes to the direct formation of xenon, and therefore to increased parasitic absorption following a local increase in flux.

Figures 4-31 and 4-32 show the power oscillations for \pm 10 percent changes in thermal absorption cross section when TURBO* was used. These changes were made at $1 / 10$ of the normal perturbation in order to reduce the size of the oscillations. These can be compared with the graph of the same core with no absorption change (refer to Figure 3-1).

Using ZEST, similar calculations were made except that in these a smaller poison was inserted. The periods and stability indices are shown in cases 27 and 28 of Appendix C. The time-step-corrected stability indices (Section 2.3.2) are also plotted in Figure 4-7 where they are compared with the parametric curves from modal analysis. As these points were produced using a power coefficient of $-3.1 \times 10^{-6} / \mathrm{MWt}$, it appears that they agree quite well with modal theory. This gives some measure of confidence in the validity of the modal curves as a means of determining core characteristics. However, the equivalent points for the oscillation period were not plotted since no effective formula for correction of period with regard to finite time-step length has as yet been developed.

The power oscillations for changes of \pm 10 percent in the total yield produced using TURBO* are shown in Figures 4-33 and 4-34. From these data and the corresponding results from ZEST as shown in cases 25 and 26 of Appendix C, it is evident that stability is more dependent on this factor than on changes in Xe-135 absorption cross section. Again, good agreement is obtained between modal theory and numerical computations. As above, the stability indices obtained from ZEST were plotted beside the corresponding modal curves and the discrepancy between the two was slight (see Figure 4-6). 


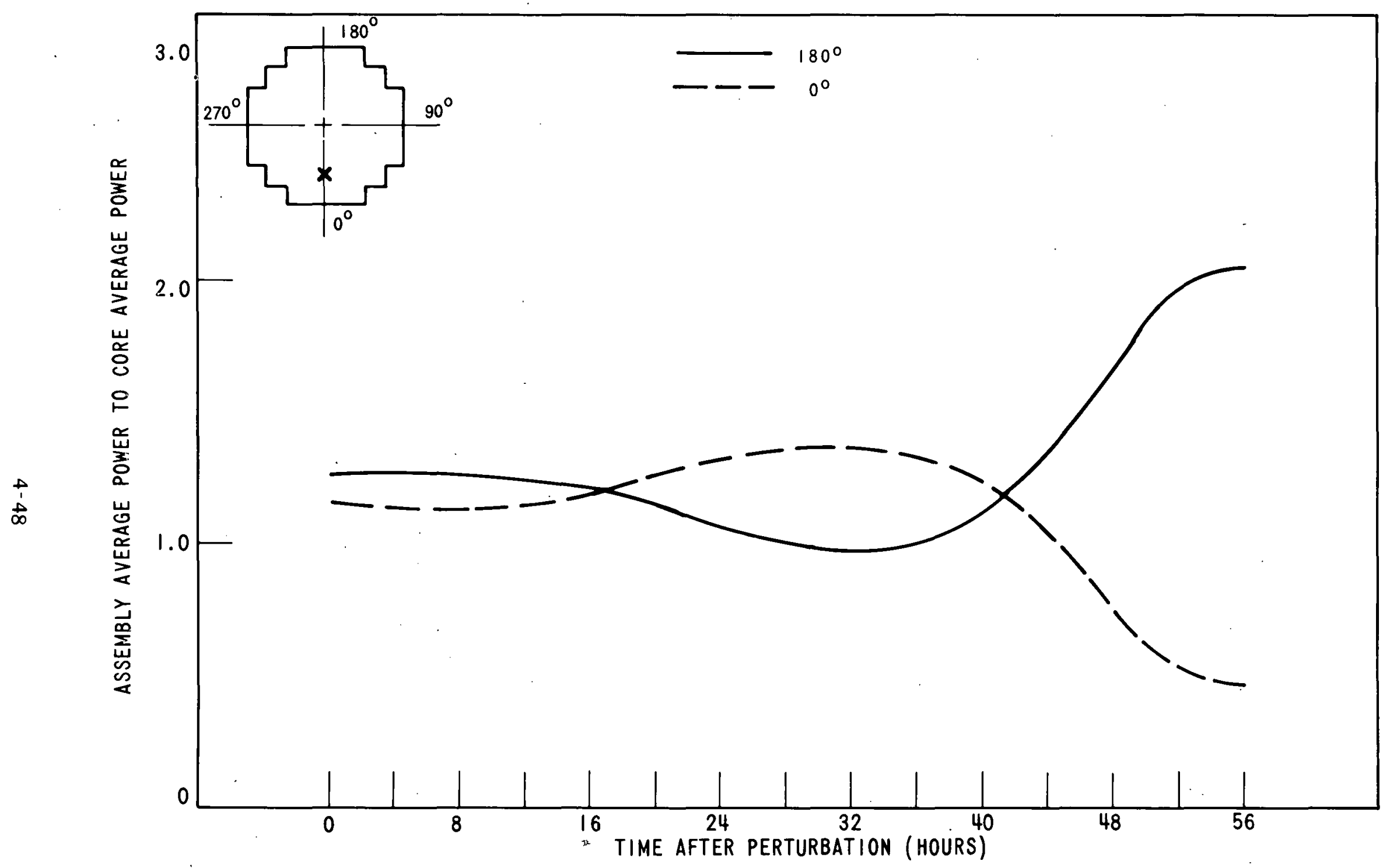

Figure 4-31. Xenon-Induced Oscillations for 10 Percent Increase in Absorption Cross-Section at a Poison Reactivity Worth of $1 \times 10^{-4}$ 


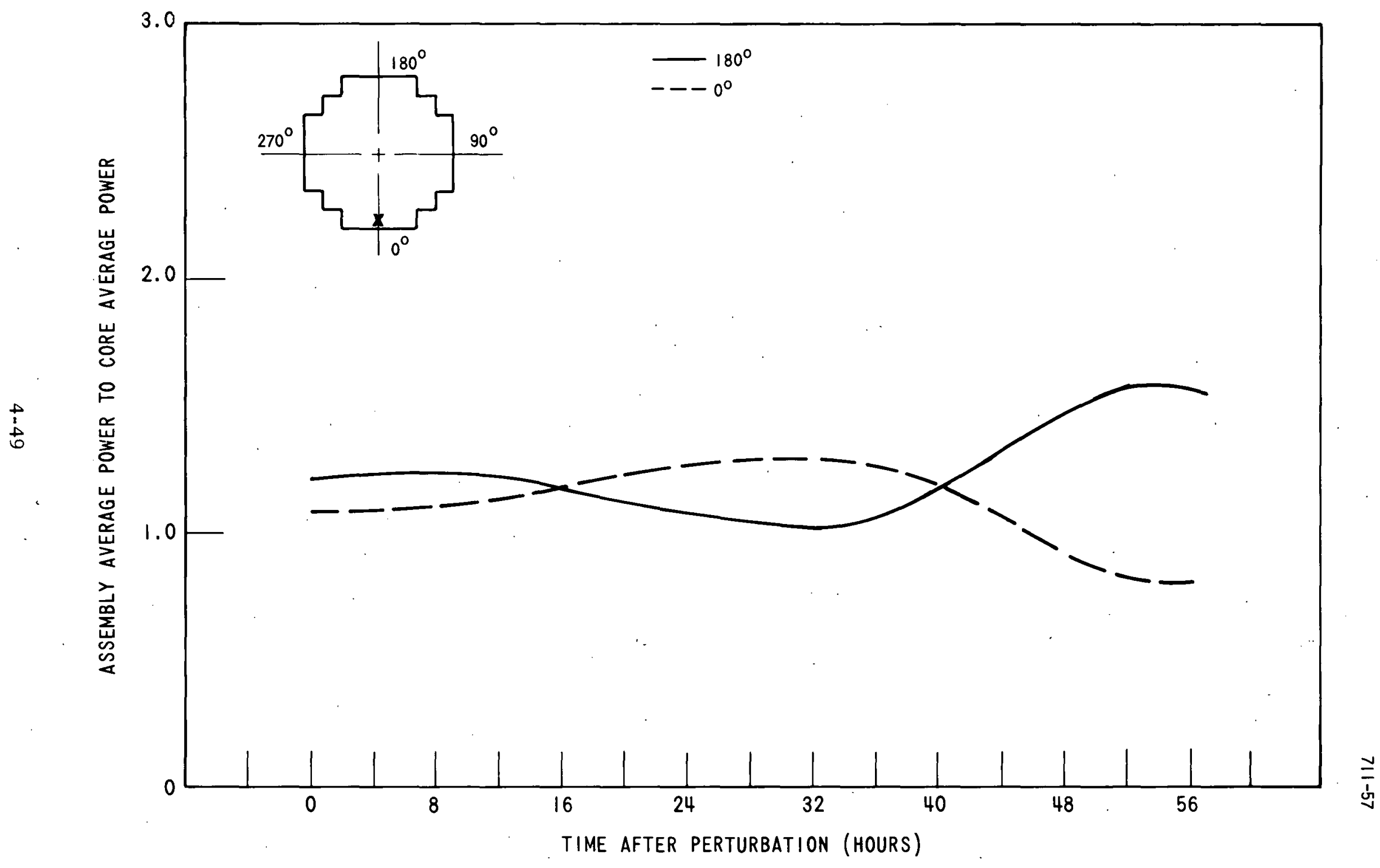

Figure 4-32. Xenon-Induced Oscillations for 10 Percent Reduction in Absorption Cross-Section at a Poison Reactivity Worth of $1 \times 10^{-4}$ 


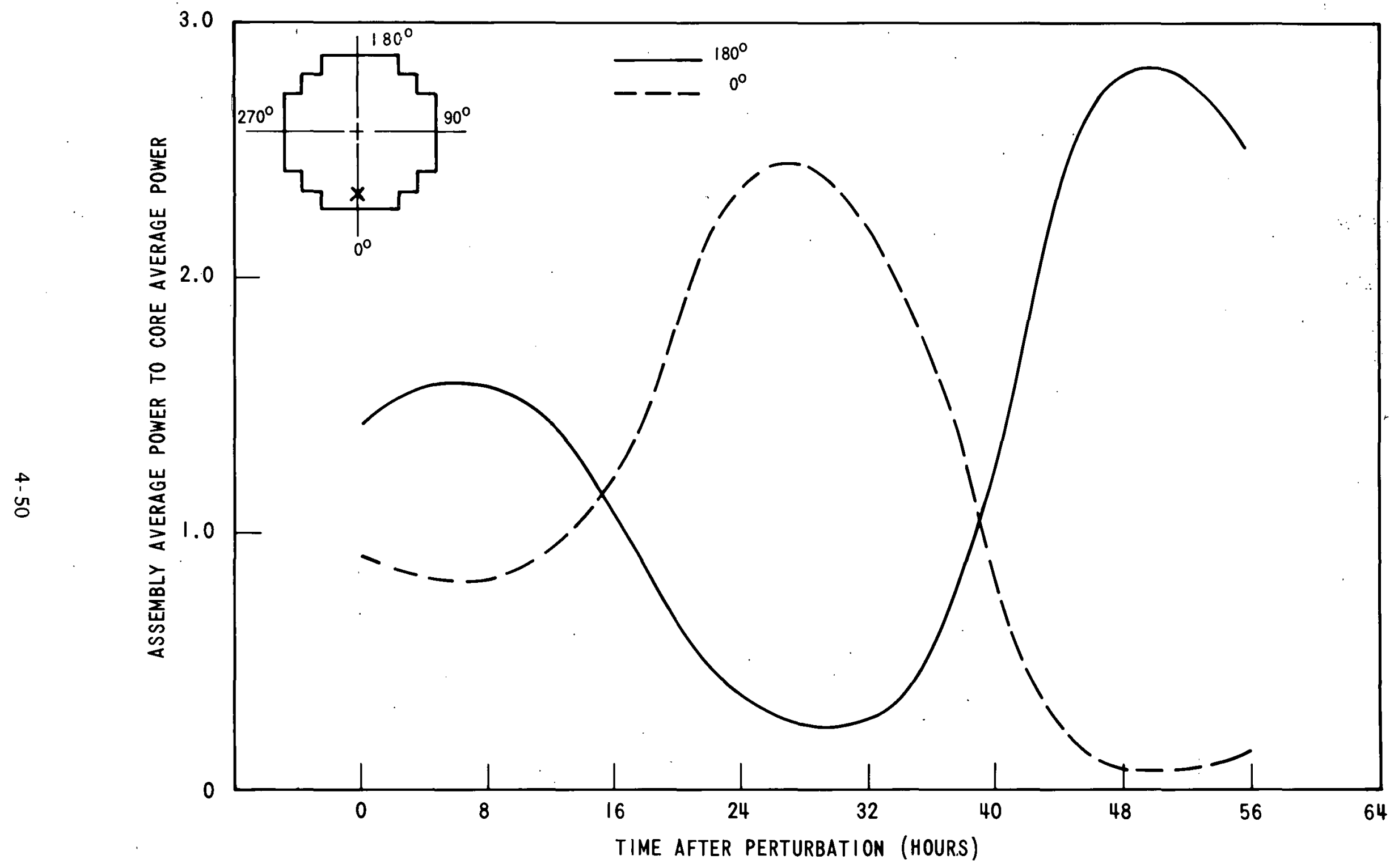

Figure 4-33. Xenon-Induced Oscillations for a 10 Percent Increase in Total Yield at a Poison Reactivity Worth of $9 \times 10^{-4}$ 


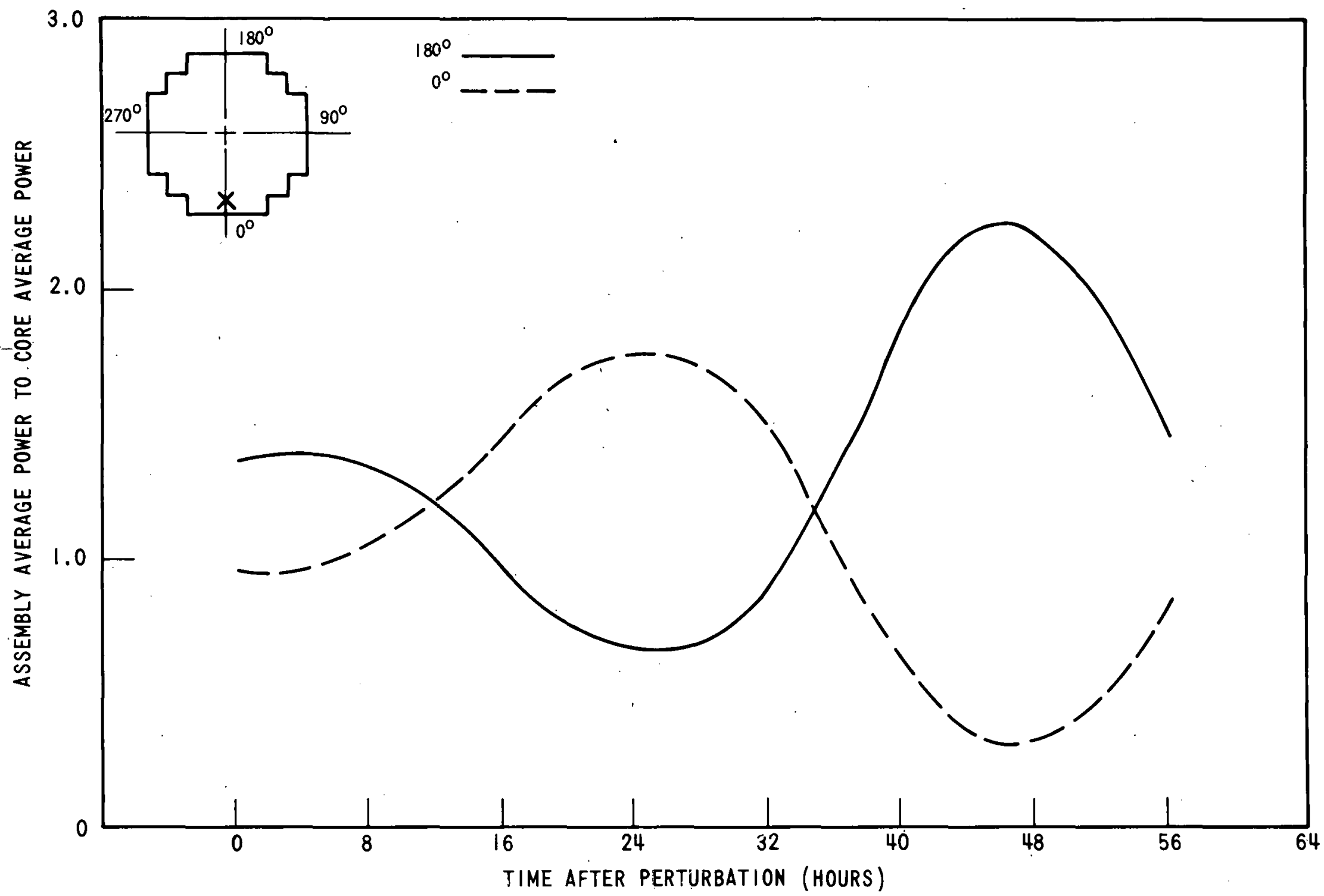

Figure 4-34. Xenon-Induced Oscillations for a 10 Percent Reduction in Total Yield at a Poison Reactivity Worth of $9 \times 10^{-4}$ 
Using TURBO*, variation of yield at $1 / 10$ of the normal perturbation was calculated and the results are shown in Figures 4-35 and 4-36. In these cases, all oscillations appear to be closer to the linear range, i.e., the exponential growth of the oscillations has not yet been saturated.

The third parameter to be varied was the direct xenon yield, an increase which should have a stablizing influence on the core. The TURBO* results for yields of 0.0 and 0.004 are shown in Figures 4-37 and 4-38, and the result for the 0.002 yield -- since. this is the value used in all other calculations -is shown in Figure 4-18. It can be seen that the stability appears to be almost completely insensitive to this parameter and its value is of little importance as far as xenon spatial stability is concerned. Both the onedimensional ZEST results and the modal analysis, however, do show a slightly increased sensitivity to this parameter although it is still of relatively minor importance. The ZEST results can be seen in cases 29 and 30 of Appendix $C$ and are also plotted for comparison with modal theory on Figure 4-8. Again, agreement between the numerical results of ZEST and modal analysis' is quite good. 


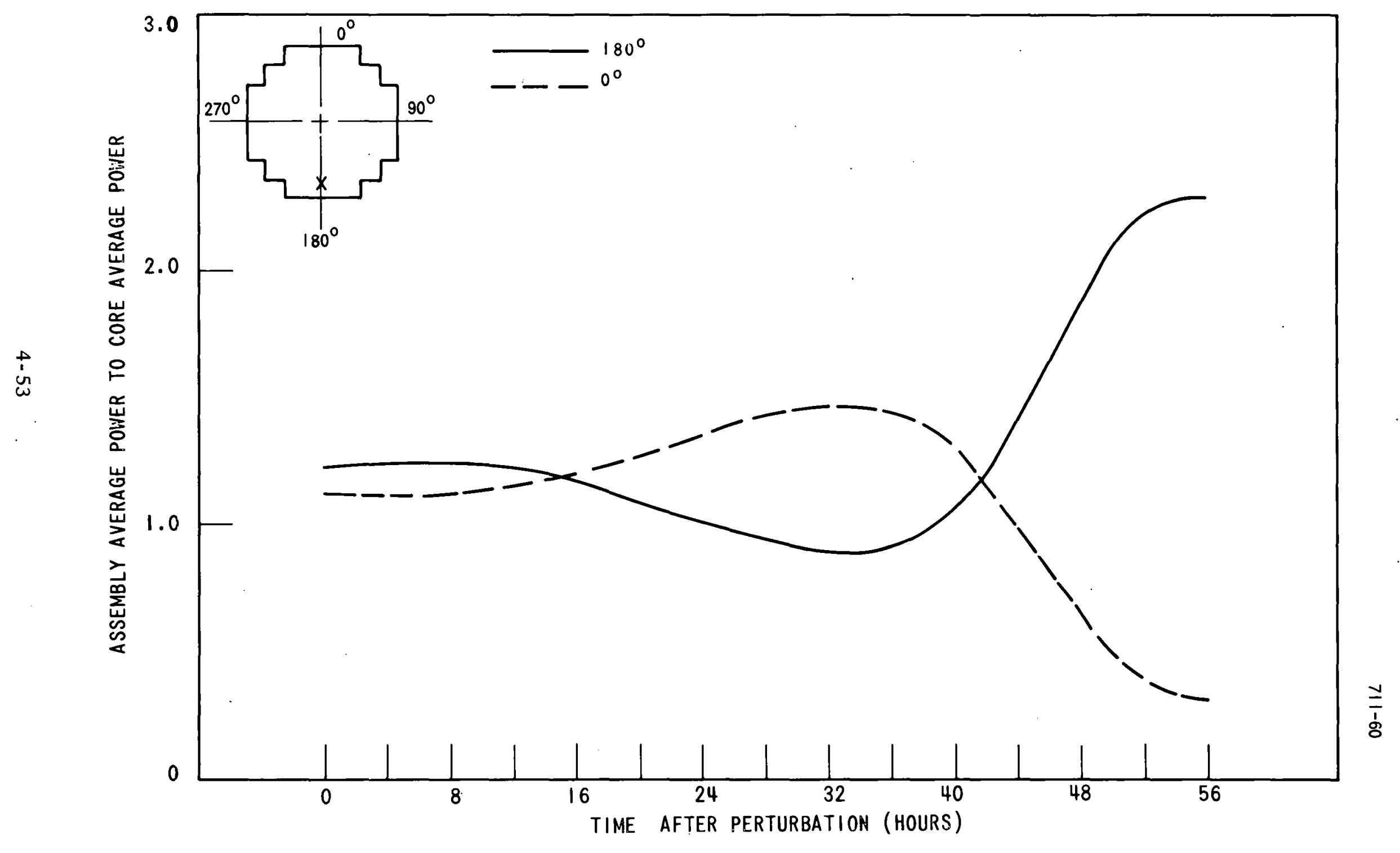

Figure 4-35. Xenon-Induced Oscillations for a 10 Percent Increase in Total Yield at a Poison Reactivity Worth of $1 \times 10^{-4}$ 


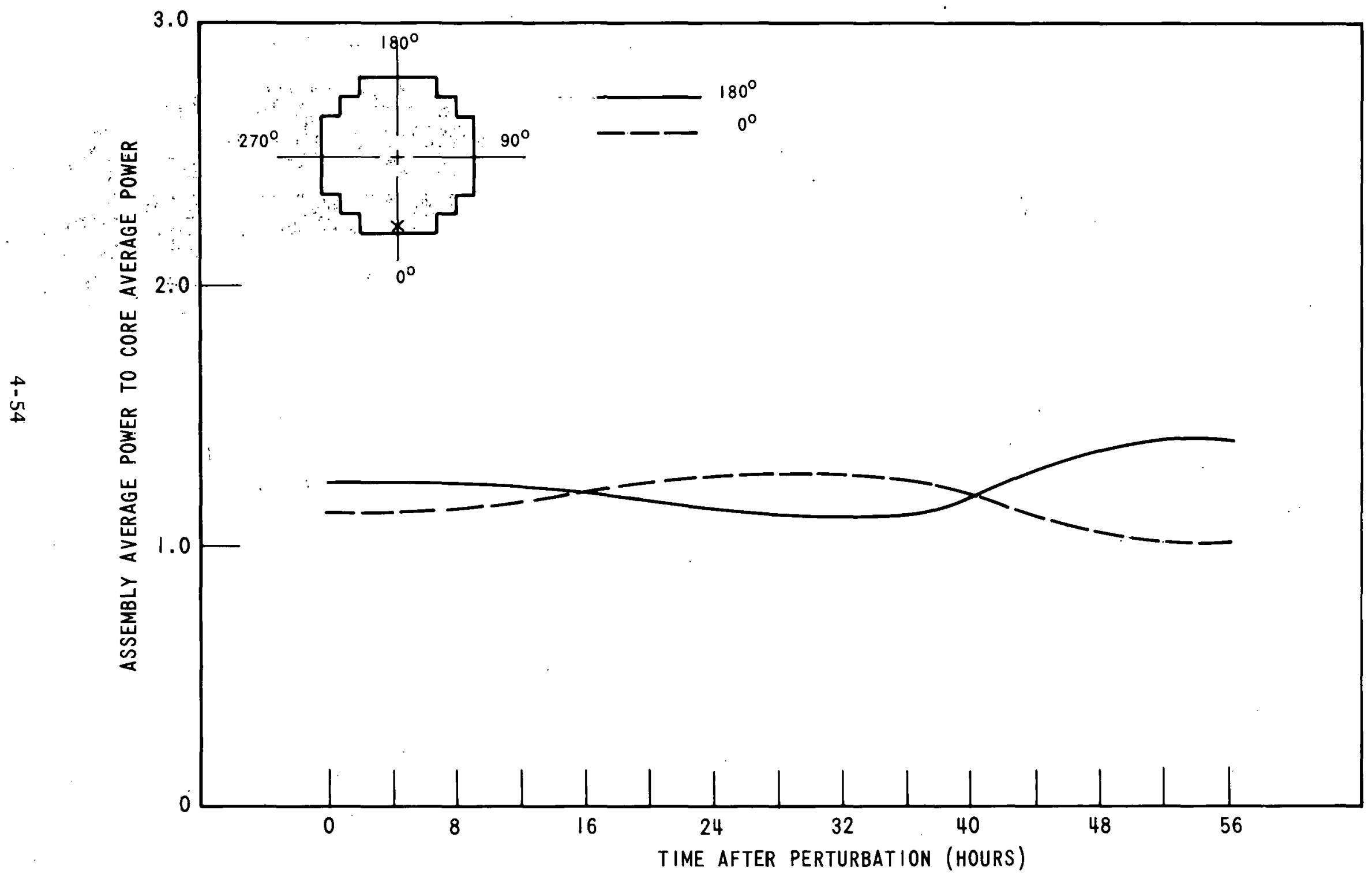

Figure 4-36. Xenon-Induced Oscillations for a 10 Percent Reduction in Total Yield at a Poison Reactivity Worth of $1 \times 10^{-4}$ 


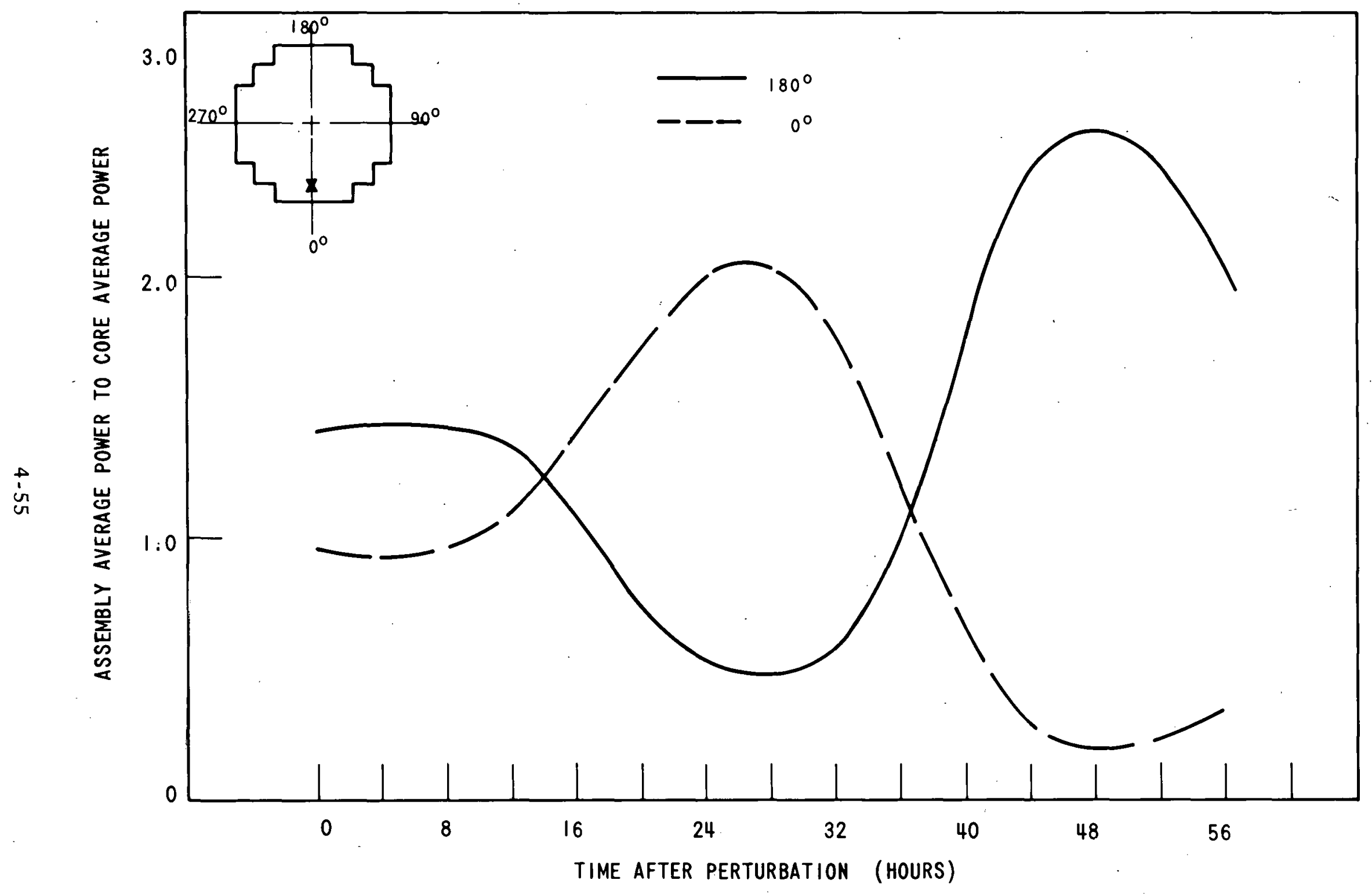

Figure 4-37. Xenon-Induced Oscillations for a Direct Xenon Yield of 0.000 


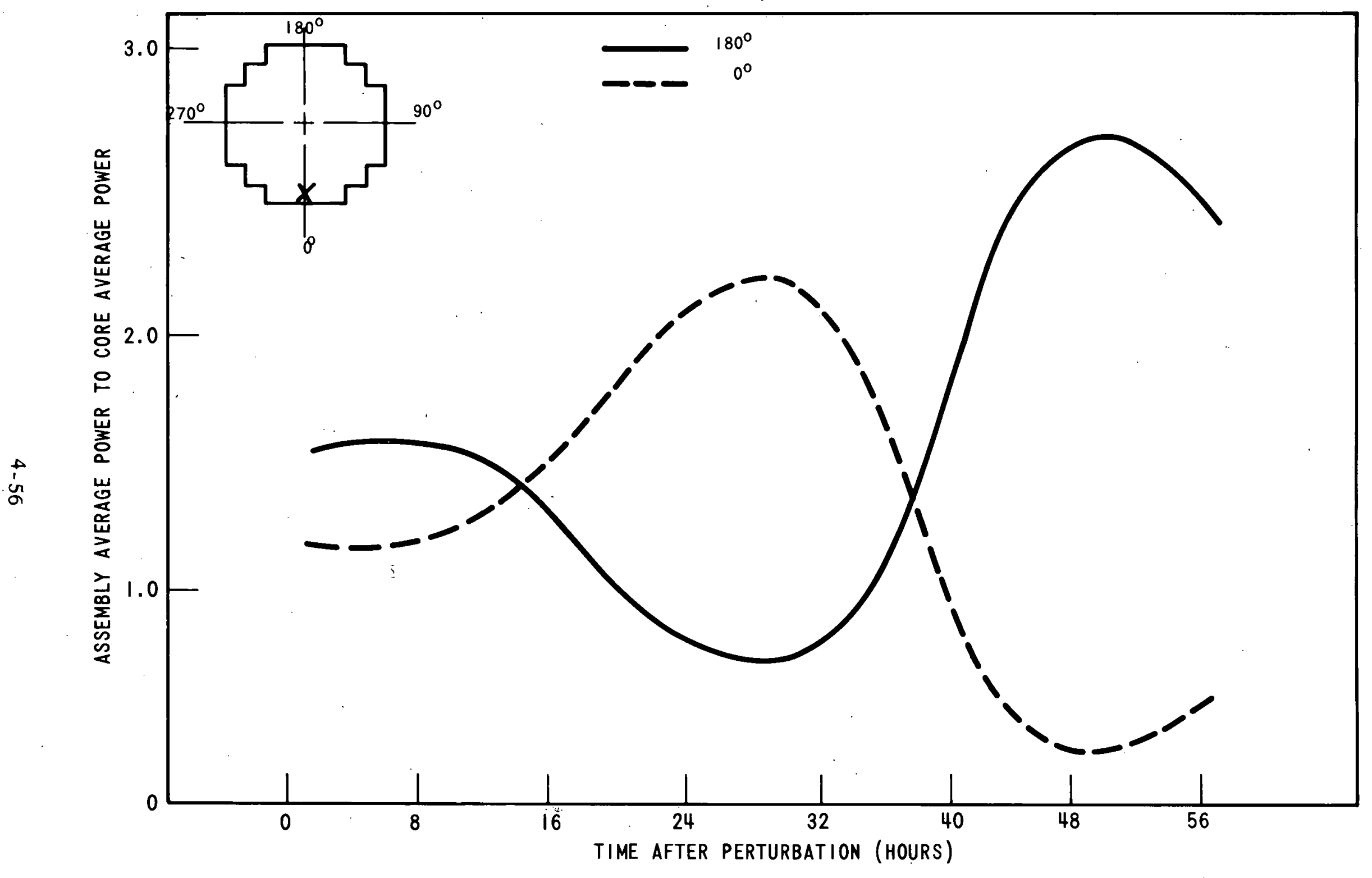

Figure 4-38. Xenon-Induced Oscillations for a Direct Xenon Yield of 0.004 
SECTION 5

SUMMARY AND CONCLUSIONS

This report has examined in detail the characteristics of free-running xenoninduced spatial instabilities in the cores of large PWR's. The analysis has been broadly divided into an evaluation of calculational methods and a study of the space-time characteristics of the oscillations in the plane perpendicular to the direction of coolant flow, including parametric and sensitivity studies. The analysis, inasmuch as it leads to a fuller understanding of the physical phenomenon of spatial xenon oscillations in PWR's, as well as to a better appreciation of the calculational methods used in stability analyses, is a necessary basis for the engineering and design analyses related to the: detection and control of spatial xenon instabilities.

In view of the geometrical complexity of large PWR's, and the importance of including realistic feedback mechanisms, the use of digital simulations in the analysis of spatial xenon instabilities is to be preferred over alternate methods. One- and two-dimensional diffusion theory codes were found to be capable of adequately exhibiting the spatial instability associated with xenon. redistribution. A number of possible sources of error were investigated within the context of digital simulations. Due to economic considerations, it is necessary to maintain computer time within reasonable limits. It was observed that the space-time behavior of the gross power distribution can be reasonably well calculated on the basis of a relatively crude spatial mesh. In a twodimensional $\mathrm{x}-\mathrm{y}$ calculation, a spatial mesh of $10 \mathrm{~cm}$ length appears to be adequate in this sense. The use of a finite, or non-zero time-step length during which the flux is taken to be constant in time can lead to significant errors in the calculated stability characteristics. A very detailed analysis of this effort was performed, leading, to a procedure for correcting numerical results for the effect of a non-zero time-step length. If no corrections are introduced, a time-step length of the order of one hour or less is required 
for adequate accuracy. It was observed that the use of a flux convergence criterion in diffusion theory calculations of the order of $10^{-4}$ (a value normally used in static flux calculations) will lead to significant errors in calculated xenon stability characteristics. A much tighter convergence criterion, of the order of $10^{-5}$, is required for reasonable accuracy.

In the treatment of fuel temperature feedback, it was observed that the nonlinear characteristics of the power coefficient affect somewhat the stability characteristics. In two-group digital simulations of xenon oscillations, it was shown that it was necessary to account for the effect of fuel temperature on both the fast absorption cross section and the fast removal cross section. The recently developed space-dependent transfer function formalism was found useful in the investigation of the effect of a finite energy mesh, the effect of delayed neutrons, and the effect of a finite time-lag between power and temperature changes. It was concluded that a two-energy group, and probably a one-energy group, are sufficient to adequately simulate xenon-induced spatial instabilities in large PWR's. The presence of delayed neutrons was shown to have no noticeable effect on the stability characteristics. In the treatment of temperature feedbacks, it was shown that the temperature time constants can be taken as zero in digital simulations of spatial xenon oscillations in large PWR's. A moderator temperature time constant of the order of a few minutes is required in order for the temperature time lag to influence the spatial xenon instability characteristics.

The linearized modal expansion theory, based on material or geometric modes, is useful in that it leads to analytical expressions for the stability characteristic. It is also helpful in gaining a better physical insight into the phenomenon of spatial instabilities. Based on such a modal theory, analytical expressions relating a spatial stability index to various core parameters and basic physics data were described. These expressions served as the basis for an extensive parametric and sensitivity analysis for large PWR's. A number of direct comparisons were performed between modal theory results and numerical results from direct digital simulations. In general, the agreement was satisfactory, thus providing some confidence in the extensive parametric modal theory results. 
Extensive digital simulations with a two-dimensional code have shown that the most readily excited mode of oscillations in the plane perpendicular to the direction of coolant flow consists of diametral oscillations. Such spatial oscillations should be relatively simple to detect and control with existing detection and control systems. It was demonstrated, however, that precessing oscillations around the horizontal plane can be excited by a particular sequence of asymmetric local perturbations. Two-dimensional contour plots have been obtained for a large number of differing core designs to explicitly illustrate the spatial characteristics of the oscillations.

It is demonstrated that, within the linear range of oscillations, the stability characteristics were relatively independent of the magnitude of the perturbation. A small effect, due to the non-linear nature of the fuel temperature feedback, was observed to detract from stability somewhat as the size of the perturbation was increased.

A series of digital calculations with perturbations of zero magnitude have shown that very small perturbations, of the order of the convergence criterion in diffusion theory calculations, will excite oscillations in an otherwise unstable core. Based on the effect of the pseudo-noise arising from the numerical calculations, it appears that the presence of a noise field will affect the stability of a core for small perturbations.

The fuel temperature feedback, which arises from the Doppler effect in a PWR, was observed to be a major stabilizing influence for azimuthal or diametral oscillations. The effect of moderator density feedback for both negative and positive moderator temperature coefficients was briefly evaluated using the space-dependent transfer function method. It was shown that the moderator temperature feedback effect must be included in calculations of spatial xenon stability index and the period of oscillations. These results were corroborated in part by more detailed digital simulations. 
REFERENCES

1. Ward, "The Problems of Flux Instability in Large Power Reactors," AECL-345 (1956).

2. Henry, A. F. and Germann, J. D., "Oscillations in the Power Distribution of a Reactor," Nuclear Science and Engineering 2 , (469-480) 1957.

3. Randa11, D. and St. John, D. S., "Criteria for Xenon Instability," Nucleonics $16,3,82-6$ (1958).

4. Pearce, R. M., "Method of Studying Xenon Spatial Instability with an Analogue Computer," Nuclear Science and Engineering 11, 328-37 (1961).

5. Strawbridge, L. E., Allard, E. C., Bhalla, C. P., "Xenon-Induced Core Instabilities," WCAP-3269-48 (February 1965).

6. Simpson, J. W. and Rickover, H. G., "Proceedings of Geneva Conference," 1958.

7. Henley, S. and Mangan, R., "TURBO* - A Two-Dimensional Few-Group Depletion Code For the IBM-7090," WCAP-6059.

8. Lellouche, G. S., "Space Dependent Xenon Oscillations," Nuclear Science and Engineering 12, 482-9 (1962).

9. Barss, W. M., "Digital Simulation of Xenon Instability in Reactors," AECL-1226 (1961).

10. Pearce, R. M. and Roth, R. E., "AG-20 Program for Studying Xenon Oscillations and Applications to CANDU," AECL-1803 (1963).

11. Poncelet, C. G., "Solution of Multigroup Space-Time Diffusion Equations," International Conference on the Utilization of Research Reactors and Reactor Mathematics and Computation, Mexico City (May 1967).

12. Morris, D. R. and Lacy, D. S., "A Simple Approximate Test for Spatial Xenon Stability," Transactions American Nuclear Society, $\underline{3},(2)$, (December, 1957). 
13. Davison, B., "Correction for Non-Zero Time-Step in the Numerical Simulation of Spatial Oscillations of Xenon in a Nuclear Reactor," AECL-1292 (1961).

14. Poncelet, C. G., "Analysis of the Reactivity Characteristics of Yankee Core I," WCAP-6050 (January 1963).

15. Chernick, J., Lellouche, G. and Wollman, W., "The Effect of Temperature on Xenon Instability," Nuclear Science and Engineering 10, 120-31 (1961).

16. Shotkin, L. M. and Abernathy, F. M., "Linear Stability of the Thermal Flux in a Reflector Core Containing Xenon and Temperature Reactivity Feedback," Nuclear Science and Engineering 15, 197-212 (1963).

17. Chernick, J., "The Dynamics of a Xenon-Controlled Reaction," Nuclear Science and Engineering 8, 233-43, (1960).

18. Wiberg, D. M., "Optimal Feedback Control of Spatial Xenon Oscillations in a Nuclear Reactor," Thesis, TID-21273 (1964).

19. Lellouche, G. S., "Reactor Size Sufficient for Stability Against Spatial Xenon Oscillations," BNL-6330 (1962).

20. Norinder, 0., "Two-Group Analysis of Xenon Stability in Slab Geometry by Modal Expansion," AE-108 (1963).

21. Randal1, D. and St. John, D. S., "Xenon Spatial Oscillations," Nuclear Science and Engineering 14, 204-06 (October 1962).

22. Mark1, H., "The Possible Instability of the Power Density and Xenon Concentration in a Large Thermal Power Reactor," Nukleonik, 2 , 90-100 (May 1960).

23. Gyorey, G. L., "The Effect of Modal Interaction on the Xenon Instability Problem," Transactions American Nuclear Society 4 , 1-83 (June 1961).

24. Gyorey, G. L., "The Effect of Modal Interaction in the Xenon Instability Problem," Nuclear Science and Engineering 13, 338-44 (August 1962).

25. Pearce, R. M., "Xenon Oscillations in Finite Reactors," Nuclear Science and Engineering 16, 336-7 (1963).

26. Kaplan, S., "The Property of Finality and the Analysis Problems and Reactor Space-Time Kinetics By Various Modal Expansions," Nuclear Science and Engineering 9, (3), 357-61 (May 1961).

27. England, T. R., Hartfield, G. L. and Deremer, R. K., "Xenon Spatial Stability in Large Seed Blanket Reactors," WAPD-TM-606 (April 1967). 
28. Ewen, R. L., "Calculation of Complex Natural Modes for Spatial Xenon Oscillations and Comparison with a Simple Approximation," Transactions American Nuclear Society $\underline{5}$, (1), 179 (June 1962).

29. Poncelet, C. G., "Space-Dependent Reactor Transfer Functions, Part I, Theory," Westinghouse Electric Corporation, WCAP-2873 (1966).

30. Poncelet, C. G., "Solution of Linearized Space- and Energy-Dependent Reactor Kinetics with Arbitrary Feedback," Transactions American Nuclear Society, $\underline{9}, 455$ (November 1966).

31. O'Boyle, M. J., et al., "Control of Xenon Instabilities in Large PWR's," Technical Progress Report for Period Ending December 1966 (WCAP-3680-2).

32. O'Boyle, M. J., et al., "Control of Xenon Instabilities in Large PWR's," Technical Progress Report for Period Ending March 1967 (WCAP-3680-3).

33. Marlowe, O. J., and Suggs, M. C., "WANDA-5 - One-Dimensional Neutron Diffusion Equation Program For the Philco-2000 Computer" (WAPD-TM-241). 
APPENDIX A

CORE DESCRIPTIONS

The PWR cores used as a basis for the calculations of xenon spatial instabilities throughout this report have been selected to correspond to current designs of large PWR's and, in the case of very large cores, to anticipated designs.

A single fuel assembly design was used for the different size cores and different fuel loadings studied in the report. The assembly is of the RCC (Rod Cluster Control) type. The basic fuel assembly design data are listed in Table $\mathrm{A}-1$.

TABLE A-1

FUEL ASSEMBLY DESIGN DATA

\begin{tabular}{|l|c|}
\hline Assembly size (in.) & $8.47 \times 8.47$ \\
Fuel length (in.) & 144 \\
Fuel rods per assembly & 204 \\
Fuel Rod O.D. (in.) & 0.422 \\
Clad Thickness (in.) & 0.243 \\
Pellet Diameter (in.) & 0.367 \\
Pitch (in.) & 0.563 \\
\hline
\end{tabular}

A typical core layout for the 11-foot (effective diameter) core is shown in Figure A-1. The figure shows a three-zone enrichment pattern. Figure 3-5 shows a typical checkerboard fuel loading pattern for the 11-foot core. Table A-2 lists the different core sizes investigated in the report, with the corresponding number of fuel assemblies comprising the fuel loading. 


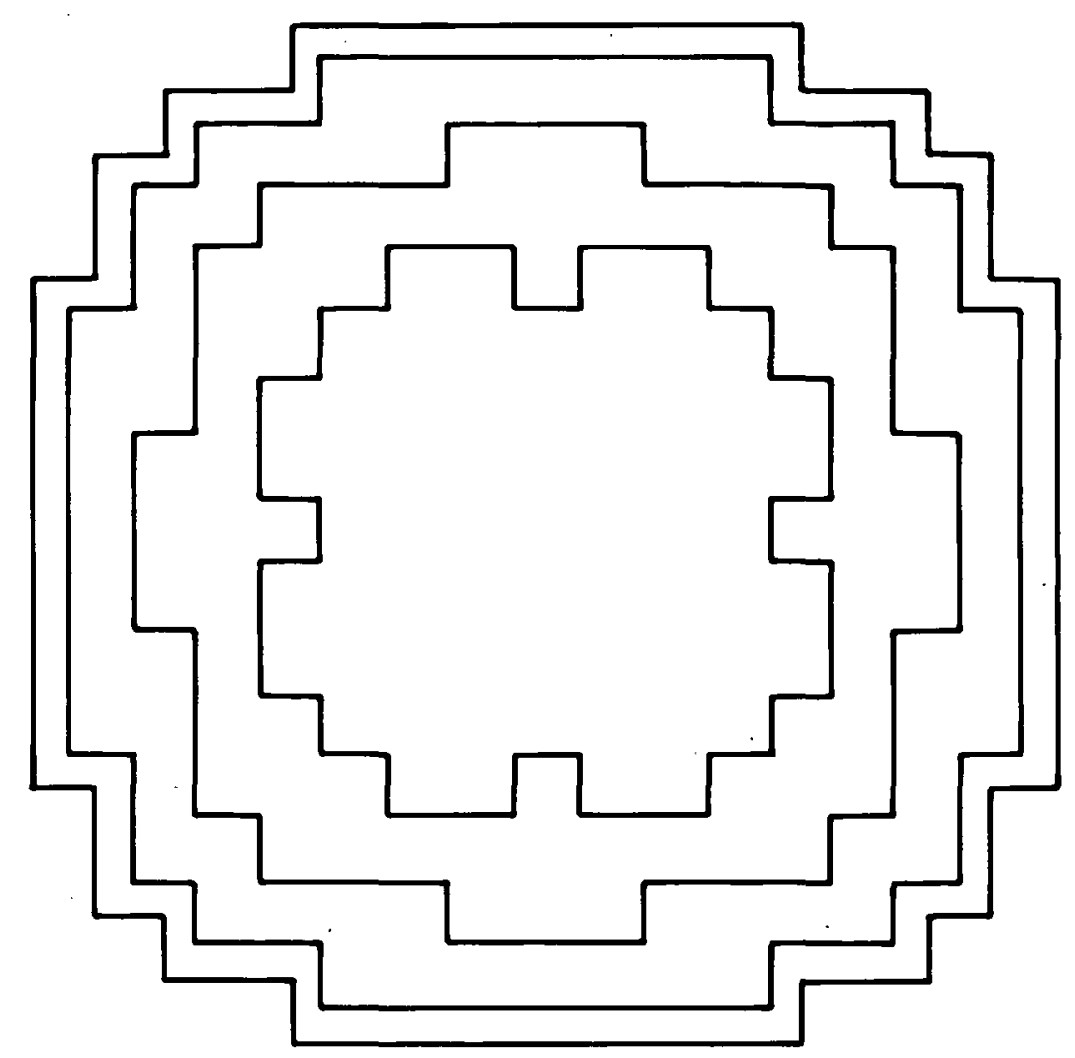

Figure A-1. Configuration of 11-Ft. Core with 3 Fueled Regions and Reflector 
TABLE A-2

CORE DIMENSIONS

\begin{tabular}{|c|c|c|c|}
\hline $\begin{array}{l}\text { Effective Core } \\
\text { Diameter (ft) }\end{array}$ & $\begin{array}{c}\text { Effective Core } \\
\text { Diameter (m) }\end{array}$ & $\begin{array}{c}\text { Number of Fuel } \\
\text { Assemblies }\end{array}$ & $\begin{array}{c}\text { Total } \\
\text { Power (MWt) }\end{array}$ \\
\hline 8.8 & 2.67 & 121 & 1733 \\
10 & 3.04 & 157 & 2246 \\
11 & 3.37 & 193 & 2760 \\
13 & 3.98 & 269 & 3850 \\
15 & 4.53 & 349 & 4987 \\
\hline
\end{tabular}

$*$ Average power density of $84.5 \mathrm{w} / \mathrm{cc}$ assumed for all cores.

All cores investigated were surrounded by a 1-1/2 inch steel baffle and an effectively infinite light water reflector.

For the sake of simplicity and consistency, the same nominal thermal-hydraulic data were assumed for all cores investigated. Table A-3 gives a list of the more important parameters. In all cores, constant and uniform core inlet conditions were assumed.

TABLE A-3

THERMAL AND HYDRAULIC DATA

\begin{tabular}{|lr|}
\hline System Pressure (psia) & 2250 \\
Inlet Coolant Temperature $\left({ }^{\circ} \mathrm{F}\right)$ & 548 \\
Average Coolant Temperature Rise $\left({ }^{\circ} \mathrm{F}\right)$ & 52.5 \\
Core Average Temperature & 577.7 \\
Total Flow Rate $\left(10^{6} \mathrm{lb} / \mathrm{hr}\right)$ & 136.4 \\
Coolant Average Velocity $(\mathrm{ft} / \mathrm{sec})$ & 16.1 \\
Average Heat Flux (Btu/hr-ft $\left.{ }^{2}\right)$ & 175,600 \\
Average Fuel Temperature $\left({ }^{\circ} \mathrm{F}\right)$ & 1520 \\
\hline
\end{tabular}

In Sections 3 and 4 of the report, certain parameters of Table A-3 were arbitrarily varied for parametric analysis purposes.

The fuel for all calculations in this report is unirradiated, slightly- 
enriched $\mathrm{UO}_{2}$. Plutonium fuels were not considered in this study. A wide range of steady-state power distributions were obtained by using different values of U-235 enrichments in a multi-zone (usually three-zone) pattern. In all calculations it was assumed that no control rods were present in the core at steady-state -- the excess reactivity being controlled by boron dissolved in the light water coolant.

The computational methods and basic cross section libraries used to prepare regionwise macroscopic cross sections for use in the one- and two-dimensional calculations were similar to those in use at the Westinghouse Atomic Power Divisions. The entire fuel assembly design, including the basic unit cell, the RCC holes and structural materials, was homogenized. Although this homogenization does not permit a detailed calculation of local power peaks, the procedure is entirely adequate for the calculation of the gross power distribution across the core and for the calculation of spatial xenon oscillations. 


\section{APPENDIX B}

DEFINITION OF THE STABILITY INDEX

The stability index, sometimes referred to as the growth or decay constant, is defined in this report assuming that the oscillations can be approximated by one mode, i.e.,

$$
\phi(x, t)=\phi_{0}(x)+\delta \phi(x, t)
$$

where

$$
\delta \phi(x, t)=\psi(x) \exp [(b+i c) t]
$$

and

$$
\phi_{0}(x) \text { is the equilibrium flux. }
$$

In this equation, $b$ is defined as the stability index. If $\delta \phi_{1}$ and $\delta \phi_{2}$ are consecutive maxima in the perturbed flux at times $t_{1}$ and $t_{2}$, then using the above equation:

$$
b=\frac{1}{t_{2}-t_{1}} \log \left(\frac{\delta \phi_{1}}{\delta \phi_{2}}\right)
$$

Because of the above assumption and -- more importantly -- because of the non-linear saturation of the flux envelope, the value of $b$ so defined is in many cases somewhat inaccurate. This is particularly so when a large perturbation is used. 
APPENDIX C

STABILITY INDEX TABLES

C-1

$\psi$ 
TABLE C-1

VARIATION OF TIME-STEP LENGTH

\begin{tabular}{|c|c|c|c|c|c|c|c|c|c|}
\hline Case & $\begin{array}{l}\text { Length } \\
\text { (feet) }\end{array}$ & $\begin{array}{c}\text { Enrichment } \\
(a / 0)\end{array}$ & $\begin{array}{l}\text { Power } \\
(w / c c)\end{array}$ & $\begin{array}{c}\text { Fuel } \\
\text { Temperature } \\
\text { Feedback }\end{array}$ & $\begin{array}{l}\text { Period } \\
\text { (hours) }\end{array}$ & $\begin{array}{l}\text { Time- } \\
\text { Step } \\
\text { (hours) }\end{array}$ & $\varepsilon_{p}$ & $\begin{array}{l}\text { Stability } \\
\text { Index } \\
\left(\mathrm{hr}^{-1}\right)\end{array}$ & $\begin{array}{c}\text { Corrected } \\
\text { Stability } \\
\text { Index } \\
\left(\mathrm{hr}^{-1}\right)\end{array}$ \\
\hline 1 & 10 & 2.5 & 84.5 & No & 32.5 & 1 & $10^{-4}$ & -0.00493 & -0.0040 \\
\hline 2 & 10 & 2.5 & 84.5 & No & 35 & 2 & $10^{-4}$ & -0.00603 & -0.0040 \\
\hline 3 & 10 & 2.5 & 84.5 & No & 44 & 4 & $10^{-4}$ & -0.01211 & -0.0040 \\
\hline 4 & 10 & 2.5 & 106 & No & 30.5 & 1 & $10^{-4}$ & 0.015865 & 0.0217 \\
\hline 5 & -10 & 2.5 & 106 & No & 35.5 & 2 & $10^{-4}$ & 0.010243 & 0.0217 \\
\hline 6 & 10 & 2.5 & 106 & No & 41.3 & 4 & $10^{-4}$ & -0.002133 & 0.0217 \\
\hline 7 & 11 & $2.4 / 2.5 / 3.0$ & 84.5 & Nominal & 33 & 1 & $10^{-4}$ & 0.01234 & 0.0160 \\
\hline 8 & 11 & $2.4 / 2.5 / 3.0$ & 84.5 & Nominal & 37 & 2 & $10^{-4}$ & 0.00764 & 0.0160 \\
\hline 9 & 11 & $2.4 / 2.5 / 3.0$ & 84.5 & Nominal & 40 & 4 & $10^{-4}$ & -0.00235 & 0.0160 \\
\hline 10 & 11 & $2.4 / 2.5 / 3.0$ & 70 & Nomina1 & 36 & 1 & $10^{-4}$ & -0.001434 & 0 \\
\hline 11 & 11 & $2.4 / 2.5 / 3.0$ & 70 & Nominal & 39 & 2 & $10^{-4}$ & -0.0028517 & 0 \\
\hline 12 & 11 & $2.4 / 2.5 / 3.0$ & 100 & Nominal & 34 & 2 & $10^{-4}$ & 0.01758 & 0.0348 \\
\hline 13 & 11 & $2.4 / 2.5 / 3.0$ & 100 & Nominal & 42 & 4 & $10^{-4}$ & 0.00245 & 0.0348 \\
\hline
\end{tabular}


TABLE $\quad C-2$

VARIATION OF PERTURBATION SIZE

\begin{tabular}{|c|c|c|c|c|c|c|c|c|c|c|}
\hline Case & $\begin{array}{l}\text { Length } \\
\text { (feet) }\end{array}$ & $\begin{array}{c}\text { Enrichment } \\
(\mathrm{a} / \mathrm{o})\end{array}$ & $\begin{array}{l}\text { Power } \\
(w / c c)\end{array}$ & $\begin{array}{c}\text { Fuel } \\
\text { Temperature } \\
\text { Feedback }\end{array}$ & $\begin{array}{l}\text { Period } \\
\text { (hours) }\end{array}$ & $\begin{array}{l}\text { Time- } \\
\text { Step } \\
\text { (hours) }\end{array}$ & $\varepsilon_{\mathrm{p}}$ & $\begin{array}{l}\text { Stability } \\
\text { Index } \\
\left(h r^{-1}\right)\end{array}$ & $\begin{array}{c}\text { Corrected } \\
\text { Stability } \\
\text { Index } \\
\left(\mathrm{hr}^{-1}\right)\end{array}$ & $\begin{array}{l}\text { Other } \\
\text { Comment }\end{array}$ \\
\hline 14 & 11 & $2.4 / 2.5 / 3.0$ & 84.5 & Nominal & 40 & 2 & $10^{-4}$ & 0.00239 & 0.0070 & 1/2 Pert. \\
\hline 15 & 11 & $2.4 / 2.5 / 3.0$ & 84.5 & Nomina1 & 37 & 2 & $10^{-4}$ & 0.00764 & 0.0160 & Full Pert. \\
\hline 16 & 11 & $2.4 / 2.5 / 3.0$ & 84.5 & Nominal & 36 & 2 & $10^{-4}$ & 0.00953 & 0.0190 & 3/2 Pert. \\
\hline
\end{tabular}


TABLE $\quad$ C-3

VARIATION OF FEEDBACK

\begin{tabular}{|c|c|c|c|c|c|c|c|c|c|}
\hline Case & $\begin{array}{l}\text { Length } \\
\text { (feet) }\end{array}$ & $\begin{array}{c}\text { Enrichment } \\
(\mathrm{a} / \mathrm{o})\end{array}$ & $\begin{array}{l}\text { Power } \\
(\mathrm{w} / \mathrm{cc})\end{array}$ & $\begin{array}{c}\text { Fuel } \\
\text { Temperature } \\
\text { Feedback }\end{array}$ & $\begin{array}{l}\text { Period } \\
\text { (hours) }\end{array}$ & $\begin{array}{l}\text { Time- } \\
\text { Step } \\
\text { (hours) }\end{array}$ & $\varepsilon_{\mathrm{p}}$ & $\begin{array}{l}\text { Stability } \\
\text { Index } \\
\left(h \dot{r}^{-1}\right)\end{array}$ & $\begin{array}{l}\text { Corrected } \\
\text { Stability } \\
\text { Index } \\
\left(\mathrm{hr}^{-1}\right)\end{array}$ \\
\hline 17 & 11 & $2.4 / 2.5 / 3.0$ & 84.5 & Nominal & 37 & 2 & $10^{-4}$ & 0.00764 & 0.0160 \\
\hline 18 & 11 & $2.4 / 2.5 / 3.0$ & 84.5 & $+20 \%$ Nominal & 37 & 2 & $10^{-4}$ & -0.00372 & 0 \\
\hline 19 & 11 & $2.4 / 2.5 / 3.0$ & 84.5 & $-20 \%$ Nominal & 36 & 2 & $10^{-4}$ & 0.02087 & 0.0332 \\
\hline 20 & 11 & $2.4 / 2.5 / 3.0$ & 84.5 & $\partial \mathrm{T}_{\mathrm{F}} / \partial \mathrm{P}=$ Constant & 37 & 2 & $10^{-4}$ & 0.0115 & 0.0220 \\
\hline 21 & 11 & $2.4 / 2.5 / 3.0$ & 84.5 & $\begin{array}{c}\partial \mathrm{T}_{\mathrm{F}} / \partial \mathrm{P}, \partial \Sigma_{\mathrm{a}} / \partial \mathrm{P} \\
\partial \Sigma_{\mathrm{R}} / \partial \mathrm{P}=\mathrm{C}\end{array}$ & 38 & 2 & $10^{-4}$ & 0.0114 & 0.0217 \\
\hline 22 & 11 & $2.4 / 2.5 / 3.0$ & 84.5 & $\Sigma_{\mathrm{R}}$ Constant & 37 & 2 & $10^{-4}$ & 0.0356 & 0.02277 \\
\hline 23 & 11 & $2.4 / 2.5 / 3.0$ & 84.5 & $\begin{array}{l}\Sigma_{a_{I}}=\frac{(1-P)}{P} \Sigma_{R} \\
\text { and } \Sigma_{R} \text { Const. }\end{array}$ & 40 & 2 & $10^{-4}$ & -0.0145 & -0.01232 \\
\hline
\end{tabular}


TABLE $\quad$ - -4

VARIATION OF CORE PARAMETERS

\begin{tabular}{|c|c|c|c|c|c|c|c|c|c|c|}
\hline Case & $\begin{array}{l}\text { Length } \\
\text { (feet) }\end{array}$ & $\begin{array}{c}\text { Enrichment } \\
(a / o)\end{array}$ & $\begin{array}{l}\text { Power } \\
(w / c c)\end{array}$ & $\begin{array}{c}\text { Fuel } \\
\text { Temperature } \\
\text { Feedback }\end{array}$ & $\begin{array}{c}\text { Period } \\
\text { (hours) }\end{array}$ & $\begin{array}{l}\text { Time- } \\
\text { Step } \\
\text { (hours) }\end{array}$ & $\varepsilon_{\mathrm{p}}$ & $\begin{array}{l}\text { Stability } \\
\text { Index } \\
\left(\mathrm{hr}^{-1}\right)\end{array}$ & $\begin{array}{c}\text { Corrected } \\
\text { Stability } \\
\text { Index } \\
\left(\mathrm{hr}^{-1}\right)\end{array}$ & $\begin{array}{l}\text { Other } \\
\text { Comment }\end{array}$ \\
\hline 24 & 11 & $2.4 / 2.5 / 3.0$ & 84.5 & Nomina1 & 37 & 2 & $10^{-4}$ & 0.00764 & 0.0160 & Standard \\
\hline 25 & 11 & $2.4 / 2.5 / 3.0$ & 84.5 & Nominal & 40 & 2 & $10^{-4}$ & -0.010706 & -0.0067 & $0.9 \times y_{T}$ \\
\hline 26 & 11 & $2.4 / 2.5 / 3.0$ & 84.5 & Nominal & 37 & 2 & $10^{-4}$ & 0.02166 & 0.0352 & $1.1 \times \mathrm{y}_{\mathrm{T}}^{1}$ \\
\hline 27 & 11 & $2.4 / 2.5 / 3.0$ & 84.5 & Nominal & 40 & 2 & $10^{-4}$ & -0.00325 & -0.0025 & $0.9 \times \sigma \mathrm{X}$ \\
\hline 28 & 11 & $2.4 / 2.5 / 3.0$ & 84.5 & Nominal & 37 & 2 & $10^{-4}$ & 0.01656 & 0.0290 & $1.1 \times$ ox \\
\hline 29 & 11 & $2.4 / 2.5 / 3.0$ & 84.5 & Nominal & 37 & 2 & $10^{-4}$ & 0.00903 & 0.0230 & $y_{x}=0$ \\
\hline 30 & 11 & $2.4 / 2.5 / 3.0$ & 84.5 & Nominal & 37 & 2 & $10^{-4}$ & 0.00629 & 0.0142 & $y_{x}=0.004$ \\
\hline
\end{tabular}


TABLE $\quad$ C-5

VARIATION OF CONVERGENCE CRITERION

\begin{tabular}{|c|c|c|c|c|c|c|c|c|c|}
\hline Case & $\begin{array}{c}\text { Length } \\
(\mathrm{feet})\end{array}$ & $\begin{array}{c}\text { Enrichment } \\
(\mathrm{a} / \mathrm{O})\end{array}$ & $\begin{array}{c}\text { Power } \\
(\mathrm{w} / \mathrm{cc})\end{array}$ & $\begin{array}{c}\text { Fuel } \\
\text { Temperature } \\
\text { Feedback }\end{array}$ & $\begin{array}{c}\text { Period } \\
\text { (hours) }\end{array}$ & $\begin{array}{c}\text { Time- } \\
\text { Step } \\
\text { (hours) }\end{array}$ & $\varepsilon_{\mathrm{p}}$ & $\begin{array}{c}\text { Stability } \\
\text { Index } \\
\left(\mathrm{br}^{-1}\right)\end{array}$ & $\begin{array}{c}\text { Corrected } \\
\text { Stability } \\
\text { Index } \\
\left(\mathrm{hr}^{-1}\right)\end{array}$ \\
\hline 31 & 11 & $2.4 / 2.5 / 3.0$ & 84.5 & Nominal & 37 & 2 & $10^{-4}$ & 0.00764 & 0.0160 \\
32 & 11 & $2.4 / 2.5 / 3.0$ & 84.5 & Nominal & 34 & 2 & $10^{-5}$ & 0.01225 & 0.0228 \\
33 & 11 & $2.4 / 2.5 / 3.0$ & 84.5 & Nominal & 35 & 2 & $10^{-6}$ & 0.01121 & 0.0254 \\
\hline
\end{tabular}


TABLE C-6

VARIATION OF MESH INTERVAL

\begin{tabular}{c|c|c|c|c|c|c|c|c|c|c|c|}
\hline Case & $\begin{array}{c}\text { Length } \\
(\text { feet })\end{array}$ & $\begin{array}{c}\text { Enrichment } \\
(\mathrm{a} / \mathrm{o})\end{array}$ & $\begin{array}{c}\text { Power } \\
(\mathrm{w} / \mathrm{cc})\end{array}$ & $\begin{array}{c}\text { Fuel } \\
\text { Feedback }\end{array}$ & $\begin{array}{c}\text { Period } \\
\text { (hours) }\end{array}$ & $\begin{array}{c}\text { Time- } \\
\text { Step } \\
\text { (hours) }\end{array}$ & $\begin{array}{c}\varepsilon \\
\mathrm{p}\end{array}$ & $\begin{array}{c}\text { Stability } \\
\text { Index } \\
(\mathrm{hr}-1)\end{array}$ & $\begin{array}{c}\text { Corrected } \\
\text { Stability } \\
\text { Index } \\
(\mathrm{hr}-1)\end{array}$ & $\begin{array}{c}\text { Other } \\
\text { Comment }\end{array}$ \\
\hline 34 & 11 & $2.4 / 2.5 / 3.0$ & 84.5 & Nominal & 37 & 2 & $10^{-4}$ & 0.00764 & 0.0160 & $2.0 \mathrm{~cm} \mathrm{mesh}$ \\
35 & 11 & $2.4 / 2.5 / 3.0$ & 84.5 & Nominal & 35 & 2 & $10^{-4}$ & 0.00679 & 0.0122 & $4.0 \mathrm{~cm} \mathrm{mesh}$ \\
36 & 10 & 2.5 & 84.5 & No & 35 & 2 & $10^{-4}$ & 0.0060 & -0.0040 & $5.0 \mathrm{~cm} \mathrm{mesh}$ \\
37 & 10 & 2.5 & 84.5 & No & 35 & 2 & $10^{-4}$ & -0.0035 & 0.0001 & $2.5 \mathrm{~cm} \mathrm{mesh}$ \\
\hline
\end{tabular}


APPENDIX D

DESCRIPTION OF PROGRAM ZEST - FORTRAN IV CODE

ZEST is a one-dimensional, two-group diffusion theory designed for the study of xenon spatial oscillations in one dimension. It is written for two groups and incorporates a fuel-temperature feedback treatment. The design of the code is such that it is simple to modify. That this has been taken advantage of can be seen in Section 2.3.8 where the code was used to investigate the accuracy of such methods as source extrapolation between time-steps, and correction of the error due to a finite time-step length. From equations (2.1) and (2.2), the diffusion equation for the one-dimensional code ZEST may be written as follows:

$$
\begin{gathered}
-\frac{I}{r^{\rho}} \frac{d}{d r}\left[D_{1}(r, t) r \frac{\rho d \phi_{1}(r, t)}{d r}\right]+\Sigma_{1}(r, t) \phi_{I}(r, t)+\Sigma_{R}(r, t) \phi_{1}(r, t) \\
+X(r, t) \sigma_{a_{1}}^{x} \phi_{1}(r, t)=\frac{1}{\lambda} \sum_{j}\left[v \Sigma_{f_{1}^{j}}^{j}(r, t) \phi_{1}(r, t)+v \Sigma_{f_{2}}^{j}(r, t) \phi_{2}(r, t)\right] \\
-\frac{1}{r^{\rho}} \frac{d}{d r}\left[D_{2}(r, t) r^{\rho} \frac{d \phi_{2}(r, t)}{d r}\right]+\Sigma_{2}(r, t) \phi_{2}(r, t)+X(r, t) \sigma_{a_{2} \phi_{2}(r, t)}(D .1) \\
=\Sigma_{R}(r, t) \phi_{1}(r, t)
\end{gathered}
$$

These equations are, of course, coupled with the xenon and iodine equations which will be described later. Some of the special terms of the equations are described below, the others being of standard notation: 


$$
\begin{aligned}
& \rho \text { : This parameter takes the value } 0,1 \text { or } 2 \text { depending } \\
& \text { upon whether the geometry is respectively slab, } \\
& \text { cylindrical or spherical. } \\
& \Sigma_{1} \text { and } \Sigma_{2} \text { : the total macroscopic cross section for groups (1) } \\
& \text { and (2) respectively. They contain the absorption } \\
& \text { and poison cross sections. } \\
& X \text { : The xenon number density } \\
& \begin{array}{l}
\sigma_{a_{1}}^{x}, \sigma_{a_{2}}^{x}: \text { The microscopic cross sections of xenon for groups } \\
1 \text { and } 2 \text { respectively }
\end{array}
\end{aligned}
$$

The summation over $j$ in the fast group is over all fissionable isotopes. The above equations are first multiplied through by $r^{p}$ and then integrated once from $r_{n-}$ to $r_{n+}$ (refer to Figure $D-1$ ) and from this result, the threepoint finite difference formula is derived. This technique is essentially that used in the one-dimensional code WANDA [33] for solving the two-group diffusion equations.

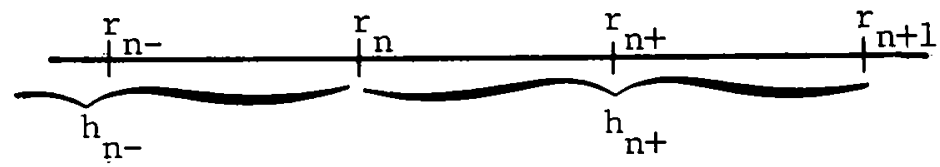

\section{Figure D-1. Finite Mesh Configuration}

The final finite difference equations are of the form

$$
a_{1, n} \phi_{1, n-1}-b_{1, n} \phi_{1, n}+c_{1, n} \phi_{1, n+1}=k_{n} \text { for the fast group }
$$

$$
a_{2, n} \phi_{2, n-1}-b_{2, n} \phi_{2, n}+c_{2, n} \phi_{2, n+1}=\sum \phi_{1, n} \text { for the thermal group }
$$

where the a's, b's, c's and $K^{\prime} s$ are of the form

$$
a_{n}=\frac{D_{n-} r_{n-}^{p}}{h_{n-}}
$$




$$
\begin{aligned}
b_{n} & =\frac{D_{n-} r_{n-}^{\rho}}{h_{n-}}+\frac{D_{n+} r_{n+}^{\rho}}{h_{n+}}+\left(\Sigma_{n--}+x_{n-} \sigma_{a}^{x}\right)\left(\frac{r_{n-1}^{\rho+1} r_{n-}^{\rho+1}}{\rho+1}\right) \\
& +\left(\Sigma_{n+}+x_{n+} \sigma_{a}^{x}\right)\left(\frac{r_{n+}^{\rho+1}-r_{n}^{\rho+1}}{\rho+1}\right) \\
c_{n}= & \frac{D_{n+} r_{n+}^{\rho}}{h_{n+}} \\
k_{n}= & s_{n-}\left(\frac{r_{n}^{\rho+1}-r_{n-}^{\rho+1}}{\rho+1}\right)+s_{n+}\left(\frac{r_{n+1}^{\rho+1}-r_{n}^{\rho+1}}{\rho+1}\right)
\end{aligned}
$$

where $S$ is the source given by the right-hand side of the group diffusion equations.

The boundary conditions are easily satisfied when the flux is zero on both boundaries as $\phi_{1}=0$ and $\phi_{n}=0$. For a reflecting condition at the origin, $\mathrm{d} \phi / \mathrm{dr}=0$ when $\mathrm{r}=0$ and

$$
\begin{aligned}
\frac{1}{r^{\rho}} \frac{d}{d r}\left[D(r) r^{\rho} \frac{d \phi}{d r}\right] & =D(r) \frac{d^{2} \phi}{d r^{2}}+\frac{d}{d r} D(r) \frac{d \phi}{d r}+\frac{\rho}{r} D(r) \frac{d \phi}{d r} \\
& =D(r)(\rho+1) \frac{d^{2} \phi}{d r^{2}}
\end{aligned}
$$

since, as $r \rightarrow 0$ and $\frac{d \phi}{d r} \rightarrow 0, \frac{1}{r} \frac{d \phi}{d r} \rightarrow \frac{d^{2} \phi}{d r^{2}}$

This latter condition can now easily be met.

Equations (D.3) and (D.4) are solved by iterating on the source and eigenvalue until these quantities have converged. The method used in solving these two equations for one iteration is as follows. Equations (D.3) and (D.4) are combined into one matrix equation as shown below 


$$
A_{n} \Phi_{n-1}-B_{n} \Phi_{n}+C_{n} \Phi_{n+1}=K_{n}
$$

where $\quad A_{n}=\left[\begin{array}{cc}a_{1 n} & 0 \\ 0 & a_{2 n}\end{array}\right], B_{n}=\left[\begin{array}{cc}b_{1 n} & 0 \\ \sigma_{r} & b_{2 n}\end{array}\right]$, and $s_{n}=\left[\begin{array}{c}K_{n} \\ 0\end{array}\right]$

The flux matrix $\Phi$ is computed from (D.5) by sweeping this equation across the mesh shown in Figure D-1.

At each iteration, the flux and power are normalized so as to maintain the total power at the (constant) input value. In so doing, this device also suppresses the fundamental mode, which is desirable when studying spatial xenon oscillations.

As was discussed in Section 2.3.6 fuel-temperature feedback is accounted for in ZEST through fitting the fast absorption and removal cross sections to the local power. This is done through the fuel temperature as an intermediate variable in the following manner:

$$
\begin{aligned}
& T_{f}=a_{0}+a_{1} P+a_{2} P^{2} \\
& \Sigma_{a_{1}}=b_{0}+b_{1} T_{f}+b_{2} T_{f}^{2} \\
& \Sigma_{R}=c_{0}+c_{1} T_{f}+c_{2} T_{f}^{2}
\end{aligned}
$$

The local xenon concentration is calculated exactly by solving the xenon and iodine equations shown below:

$$
\begin{gathered}
\frac{d I}{d t}=\sum_{j}\left[\left[y_{I_{1}}^{i} \sum_{f_{1}}^{j} \phi_{1}+y_{I_{2}}^{j} \sum_{f_{2}}^{j} \phi_{2}\right]-\lambda_{I} I\right. \\
\frac{d x}{d t}=\sum_{j}\left[\left[y_{x_{1}}^{j} \sum_{f_{1}}^{j} \phi_{1}+y_{X_{2}}^{j}\left[\sum_{f_{2}}^{j} \phi_{2}\right]\right]+\lambda_{I} I-\left[\left[\lambda_{x}+\sigma_{a_{1}}^{x} \phi_{1}+\sigma_{a_{2}}^{x} \phi_{2}\right] x\right.\right.
\end{gathered}
$$


Except for the calculations of the equilibrium flux, these latter equations are solved when final convergence for a time-step has been reached. The local xenon number densities are then used in the calculation for the flux of the next time-step. It is to be noted that the solution of these equations depends upon the flux remaining constant through this period. As has been shown in Section 2.3.2 this can lead to erroneous results.

For quicker convergence to the correct flux during a time-step, the source guess at each iteration is computed using an extrapolation technique. If $s_{n}\left(x_{i}\right)$ is the source for the $n^{\text {th }}$ iteration at position $x$ : and $s_{n-1}\left(x_{i}\right)$ the source for the $(n-1)^{\text {th }}$ iteration, then the source guess for the $(n+1)$ th iteration for the point $x_{i}$ is given by

$$
s_{n+1}\left(x_{i}\right)=s_{n}\left(x_{i}\right)(1+\theta)-\theta s_{n-1}\left(x_{i}\right)
$$

An optimum value of $\theta$ can be found using over-relaxation techniques, [33] although a simpler and quite satisfactory approach is to give $\theta$ a constant value over the complete calculation. The usual value of $\theta$ is 0.8 and, in lieu of calculating $\theta$ by the more exact over-relaxation technique, this approach was used.

At the end of each iteration in the calculation of the equilibrium flux, the corresponding equilibrium xenon is computed by equating $\mathrm{dX} / \mathrm{dt}$ and $\mathrm{d} / \mathrm{dt}$ to zero in equations (D.7) and (D.8). These new xenon number densities are then used in the next iteration.

A block diagram of the program is given in Figure D-2. Each block represents a subroutine. A description of the diagram is given below.

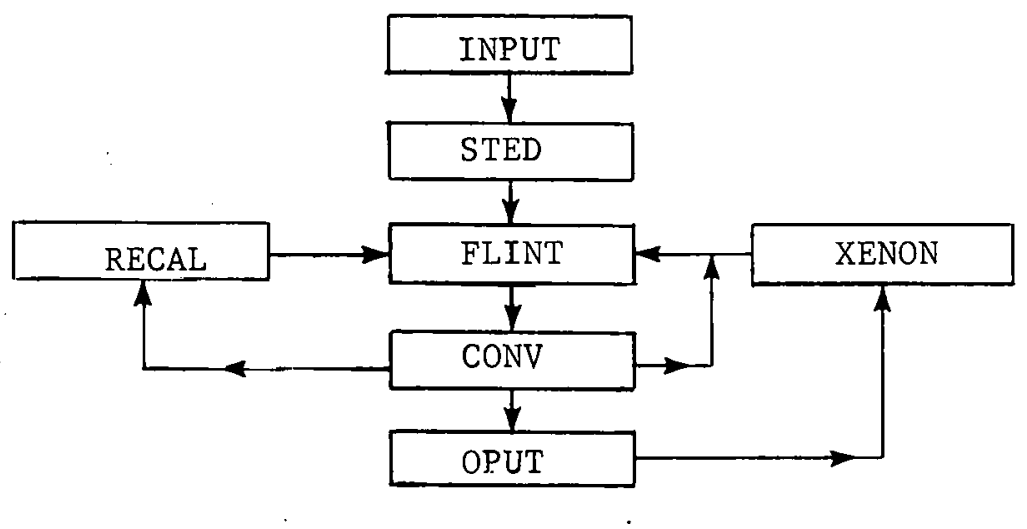

Figure D-2. ZEST Program - Block Diagram 
INPUT: This subroutine inputs the data, performs some initial calculations, and outputs the input data.

STED: This calculates the coefficients of equation of (D.5) for the steady-state case. Coefficients " $A$ " and " $C$ " are kept constant throughout all time-steps.

FLINT: With coefficients for the equation (D.5) calculated, FLINT sweeps the mesh to find the new fluxes.

CONV: The two previous source terms and eigenvalues are. compared for convergence.

RECAL: This recalculates the " $B$ " term of equation (D.5) for xenon and fuel-temperature feedbacks.

XENON: The xenon and lodine number densities for the following time-step are calculated in this subroutine.

OPUT: Subroutine for output of results. These results consist of the converged eigenvalue, the fast and thermal fluxes, power, fuel temperature, xenon concentration and source value.

ZEST is programmed such that only one set of data is required for the complete calculation. In other words, the number of time-steps after the removal of the perturbation is specified and no additional data are required for each successive time-step. 
ACKNOWLEDGEMENT

It is a pleasure for the authors to acknowledge the invaluable assistance provided by Mr. Thomas F. Farrell in the preparation and manipulation of countless input data, the running of the computer codes, and the timeconsuming reduction of output data as reported throughout this study. 